\title{
Radiation imaging with caesium bromide storage phosphors
}

by

Nicola Maree Winch

\author{
A thesis \\ submitted to the Victoria University of Wellington \\ in fulfilment of the requirements for the degree of \\ Doctor of Philosophy \\ in Physics
}

Victoria University of Wellington

2013 



\begin{abstract}
This thesis is centred on the development of a new method to prepare semitransparent $\mathrm{CsBr}: \mathrm{Eu}^{2+}$ imaging plates for high resolution $\mathrm{X}$-ray radiography. Methods of characterising the performance of these plates, and their application to dual energy imaging and neutron imaging are discussed.

The basic preparation method, based on high-pressure uniaxial compression of powder mixtures of $\mathrm{CsBr}$ and $\mathrm{EuBr}_{2}$, produces imaging plates which show good transparency and resolution. These imaging plates have a conversion efficiency of $1.5 \mathrm{pJ} \mathrm{mR} \mathrm{mm}^{-1} \mathrm{~mm}^{-3}$ compared to $5.1 \mathrm{pJ} \mathrm{mR}^{-1} \mathrm{~mm}^{-3}$ for a commercial needle imaging plate. Water is found to play a critical role in the photostimulated luminescence activation in $\mathrm{CsBr}: \mathrm{Eu}^{2+}$ storage phosphors, and imaging plates subsequently hydrated at room temperature have an increased conversion efficiency of up to $11 \mathrm{pJ} \mathrm{mR}^{-1} \mathrm{~mm}^{-3}$, better than the commercial material. A model has been suggested for the generation of the PSL active site in the imaging plates based on thermomechanical sintering and water-induced crystal regrowth.

A precise method for determining the conversion efficiency and stimulation energy of X-ray storage phosphor materials using an integrating sphere has been developed and used to characterise the materials developed in this thesis. A novel read-out method for storage phosphor imaging plates based on flood illumination and a semi-professional digital camera has also been developed and tested. Good quality X-ray images are obtained and the method shows excellent promise as a low-cost, portable $X$-ray imaging system.

A stratified detector using CsBr imaging plates has been developed for use in dual-energy imaging. Results suggest that it is possible to perform dual-energy imaging with this structure.

$\mathrm{CsBr}: \mathrm{Eu}^{2+}$ imaging plates have been produced with added neutron converters for use as thermal neutron imaging plates. An imaging plate with $5 \%{ }^{10} \mathrm{~B}_{2} \mathrm{O}_{3}$ added as a neutron converter has a PSL output $50 \%$ that of a commercial neutron imaging plate. Neutron imaging with these imaging plates has been successfully demonstrated.
\end{abstract}





\section{Acknowledgements}

Firstly, I would like to thank my supervisor Andy Edgar, for giving me the opportunity to do this project. Your guidance, support and encouragement throughout was invaluable. I also thoroughly enjoyed all our coffee breaks, lunches and especially the kayaking trips. I would also like to acknowledge my second supervisor Murray Bartle for sharing his extensive knowledge and for facilitating the ANSTO visits.

I am grateful for the assistance from the technical staff in the School of Chemical and Physical Sciences. Particularly Alan Rennie, Manu Pouajen-Blakiston and Nick Grinter in the mechanical workshop, and Rod Brown and Tim Exley in the electronics workshop. Thanks to the late Scotty Forbes for all his computer knowledge and for the semi-regular gossip sessions which are greatly missed. I acknowledge financial support from the Ministry of Science and Innovation/Ministry of Business, Innovation and Employment, in the form of a PhD stipend, and from the Victoria University Science faculty for a travel grant. I also acknowledge the Australian Institute of Nuclear Science and Engineering for facilitating and funding visits to the ANSTO nuclear reactor.

Thank you to my fellow students and colleagues for making my studies enjoyable. To my office buddy Laura, thanks for all the laughs, sunbathing, "work" trips and the occasional work help. Thanks to the Wednesday football gang for all the entertaining lunchtime football sessions.

To my family and friends, thank you for your support and encouragement throughout my studies. Thanks to Steve for help with electronic problems and stitching together X-ray images. Mum and Lindz, thanks for keeping me well fed and looking after my house when I was too busy to do it myself. And finally, thank you to Dave for your encouragement and support during the final stages of this work. 



\section{Contents}

\begin{tabular}{lll}
\hline & Introduction & 1
\end{tabular}

\begin{tabular}{|lll}
2 & Background & 9
\end{tabular}

2.1 Ionising radiation $\ldots \ldots \ldots \ldots$. . . . . . . . . . . . . 9

$2.1 .1 \quad$ X-rays $\ldots \ldots \ldots \ldots$

2.1 .2 Neutrons . . . . . . . . . . . . . . . . 13

2.2 Luminescence mechanisms . . . . . . . . . . . . . . . . 15

2.2 .1 Photoluminescence . . . . . . . . . . . . . . 15

2.2 .2 Photostimulated luminescence . . . . . . . . . . . . 18

2.3 Storage phosphor imaging plates . . . . . . . . . . . . . . 19

$2.3 .1 \quad$ BaFBr imaging plates . . . . . . . . . . . . . . . 19

2.4 Caesium bromide imaging plates . . . . . . . . . . . . . 22

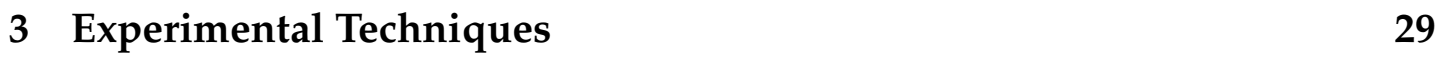

$3.1 \quad$ Sample preparation . . . . . . . . . . . . . . . . . . . . . 29

3.2 X-ray sources $\ldots \ldots \ldots \ldots$. . . . . . . . . . . . . . . .

3.3 Integrating sphere measurements of photostimulated luminescence 31

3.3 .1 Measurement process . . . . . . . . . . . 34

3.3 .2 Integrating spheres . . . . . . . . . . . . . . . 39

3.3 .3 Analysis . . . . . . . . . . . . . . . . 4 41

3.4 Determining imaging plate resolution $\ldots \ldots \ldots$. . . . . . . 45

3.5 Optical absorption measurements . . . . . . . . . . . . . . 47

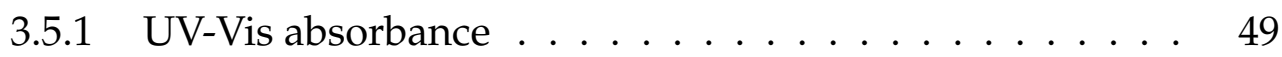


3.5 .2 Infrared spectroscopy $\ldots \ldots \ldots \ldots$. . . . . . . 50

3.6 Photoluminescence . . . . . . . . . . . . . . . . . . . . 52

$3.7 \quad$ Electron paramagnetic resonance . . . . . . . . . . . . . . 54

3.8 Scanning electron microscopy $\ldots \ldots \ldots$. . . . . . . . . . 57

4 Digital camera read-out system $\quad 61$

4.1 Technique $\ldots \ldots \ldots \ldots \ldots$. . . . . . . . . . . . . 62

4.2 Spectral sensitivity . . . . . . . . . . . . . . . . 64

4.3 Image processing $\ldots \ldots \ldots 66 . \ldots \ldots$

4.4 Spatial resolution . . . . . . . . . . . . . . . . 67

4.5 Sensitivity to X-ray dose . . . . . . . . . . . . . 70

4.6 Read-out properties . . . . . . . . . . . . . . . . . 71

4.7 Detective quantum efficiency $\ldots \ldots \ldots$. . . . . . . . . 73

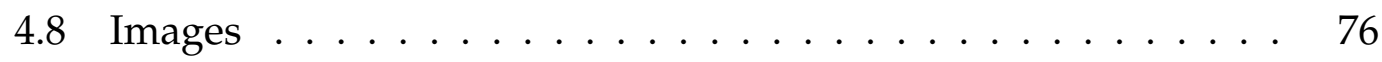

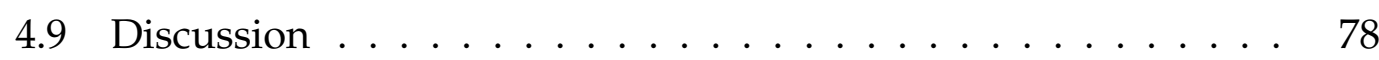

5 Synthesis of transparent CsBr:Eu ${ }^{2+}$ imaging plates 81

5.1 Preliminary observations . . . . . . . . . . . . . 83

5.2 Method 1: Milling, pressing and hydrating . . . . . . . . . . 85

5.3 Method 2: Milling, hydrating and pressing . . . . . . . . . . . 94

5.3 .1 Room air hydration . . . . . . . . . . . . . . . . 94

5.3 .2 Saturated air hydration . . . . . . . . . . . . 98

5.4 Method 3: Milling, pressing, and annealing . . . . . . . . . . 101

5.5 Method 4: Milling, annealing and pressing . . . . . . . . . . 110

5.5 .1 Room air hydration . . . . . . . . . . . . . . . . . 110

5.5 .2 Saturated air hydration . . . . . . . . . . . . 114

5.6 Method 5: Sintering, milling, pressing and hydrating . . . . . . 118

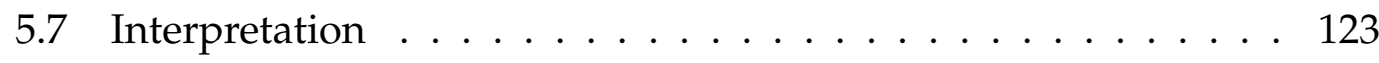

5.7 .1 Model for generation of the active PSL site . . . . . . . . 123

5.7 .2 Spatial resolution . . . . . . . . . . . . . . . . 127 
5.8 Comparison of processes . . . . . . . . . . . . . . . . . . 129

5.9 Examples of images . . . . . . . . . . . . . . . . . 131

5.10 Conclusions . . . . . . . . . . . . . . . . . 131

$6 \quad$ Dual-energy imaging with stratified CsBr imaging plates 135

6.1 Dual-energy algorithms . . . . . . . . . . . . . . 138

6.2 Dual energy imaging with doped CsBr imaging plates . . . . . . 145

6.2.1 Possible dopants for colour-separable PSL . . . . . . . . 147

6.2 .2 Imaging . . . . . . . . . . . . . . 150

6.3 Discussion . . . . . . . . . . . . . . . . . 153

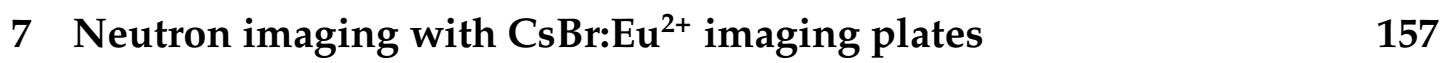

7.1 OPAL research reactor $\ldots \ldots$. . . . . . . . . . . . . 160

7.2 CsBr neutron imaging plates . . . . . . . . . . . . . . 162

$7.2 .1 \quad$ First visit . . . . . . . . . . . . . . . . 163

7.2 .2 Second visit . . . . . . . . . . . . . . . . 167

7.3 Discussion . . . . . . . . . . . . . . . . . . 174

\begin{tabular}{lll}
\hline & Summary and outlook & 177
\end{tabular}

\begin{tabular}{lr}
\hline References & 183
\end{tabular}

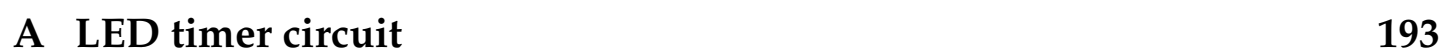

\begin{tabular}{ll}
\hline B Modulation transfer function & 197
\end{tabular}

B.1 Optical MTF for the camera system . . . . . . . . . . . . . . . . . 197

B.2 Fitting data with a sine wave . . . . . . . . . . . . . 199

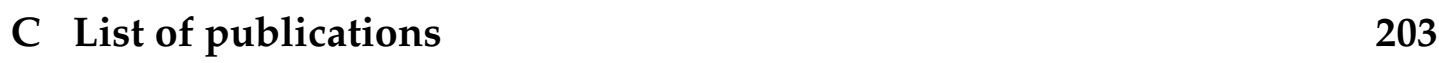





\section{List of Figures}

1.1 Attenuation coefficient versus energy for tissue, bone and lead . 2

1.2 Thermal neutron attenuation coefficient for most elements . . . 3

1.3 Demonstration of a projection X-ray image . . . . . . . . . 3

1.4 General description model for digital X-ray technologies . . . . 5

1.5 Comparison of storage phosphors, film and digital imaging systems in terms of spatial resolution and sensitivity $\ldots \ldots 6$

2.1 Example of a tungsten X-ray spectrum showing characteristic X-rays and bremsstrahlung . . . . . . . . . . . . . 10

2.2 Mass attenuation coefficients as a function of X-ray energy for aluminium and lead $\ldots \ldots \ldots \ldots 1$

2.3 Schematic of photoelectric process . . . . . . . . . . . 12

2.4 Schematic of Compton scattering . . . . . . . . . . . . . 13

2.5 Configuration-coordinate model and vibrational wavefunctions 17

2.6 Schematic of imaging with storage phosphor imaging plates . . 19

2.7 BaFBr crystal structure . . . . . . . . . . . . . . 20

2.8 BaFBr crystal structure showing $\mathrm{F}$ and $\mathrm{V}_{\mathrm{k}}$ centres . . . . . . . 20

2.9 SEM image of BaFBr:Eu ${ }^{2+}$ imaging plate and schematic of scattering within imaging plate . . . . . . . . . . . . . 21

2.10 Schematic of thermal vapour deposition technique used for nee-

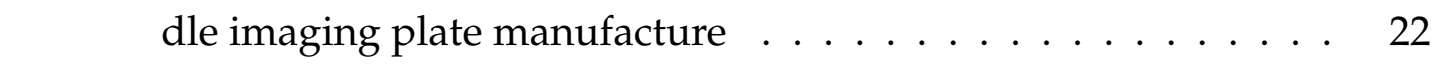

2.11 SEM images of $\mathrm{CsBr}: \mathrm{Eu}^{2+}$ imaging plate . . . . . . . . . . 23

2.12 Normalised emission and stimulation spectra for $\mathrm{CsBr}_{\mathrm{Bu}}{ }^{2+}$. . 23 
2.13 Models for the AA-EPR I and AA-EPR II centres . . . . . . . . 26

$3.1 \quad$ Equipment used to mill powders to less than $25 \mu \mathrm{m} \ldots \ldots$

3.2 Americium-241 radioactive source schematic . . . . . . . . . . . 31

3.3 Integrating sphere measurement system . . . . . . . . . . 33

3.4 Integrating sphere components . . . . . . . . . . . . 35

3.5 Dose rate as a function of distance from source to sample . . . . 38

3.6 Example of a PSL decay curve . . . . . . . . . . . . . . . . 38

$3.7 \quad$ Visual representations of power into unit solid angle and integral over surface area $\ldots . . \ldots \ldots$

$3.8 \quad$ Visual representation of power emitted from area $d A$ falling on a detector. . . . . . . . . . . . . . . . . . 41

3.9 LED power versus total count for a $405 \mathrm{~nm}$ LED $\ldots \ldots \ldots$. . . . 43

3.10 Determination of the contrast modulation and contrast transfer function by imaging a test grid . . . . . . . . . . . . . 46

3.11 Reflection, absorption and scattering of electromagnetic radiation in matter . . . . . . . . . . . . . . . . . . . . 48

3.12 Shimadzu UV-Vis 2100 spectrophotometer schematic . . . . . . 50

3.13 Michelson interferometer schematic . . . . . . . . . . . . 51

3.14 Illustration of the formation of a polychromatic light source in-

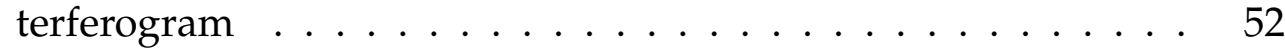

3.15 Horiba Jobin Yvon Fluorolog 3 schematic . . . . . . . . . . . 53

3.16 EPR resonance transition and absorbance and first derivative spectrum. . . . . . . . . . . . . . . . 56

3.17 EPR spectrometer $\ldots \ldots \ldots \ldots \ldots$

3.18 Schematic of SEM . . . . . . . . . . . . . . . . . 58

$4.1 \quad$ Digital camera imaging system $\ldots \ldots \ldots \ldots$. . . . . . . . 62

$4.2 \quad$ Bayer filter pattern used on the Canon 5D MkII . . . . . . . . 65 
4.3 Spectral sensitivity of the camera sensor's blue, green and red channels, PSL emission of $\mathrm{CsBr}^{-\mathrm{Eu}^{2+}}$ and $\mathrm{BaFBr}^{2} \mathrm{Eu}^{2+}$, and stimulating LED and BG12, BG3 filter characteristics . . . . . . . . . 66

$4.4 \quad$ Koren lens test chart and Nuclear Associates grating phantom used for resolution measurements . . . . . . . . . . . . . . 68

4.5 Optical and X-ray MTFs for $\mathrm{CsBr}_{\mathrm{Eu}}{ }^{2+}$ and $\mathrm{BaFBr}: \mathrm{Eu}^{2+}$ obtained by the grating method for the CMOS based system and the CCD based system . . . . . . . . . . . . . . . . . . 69

4.6 Mean pixel count versus X-ray dose for $\mathrm{CsBr}_{\mathrm{Eu}}{ }^{2+}$ and $\mathrm{BaFBr}: \mathrm{Eu}^{2+}$

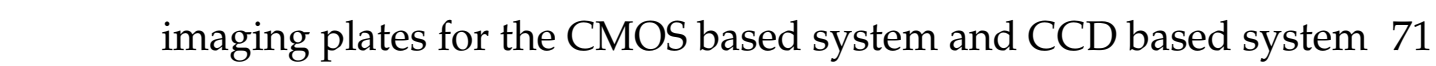

4.7 Mean dark count per pixel and standard deviation as a percentage of full scale versus shutter time for the CMOS based system and CCD based system . . . . . . . . . . . . . . 72

4.8 Fraction of image read out for $\mathrm{CsBr}: \mathrm{Eu}^{2+}$ and $\mathrm{BaFBr}: \mathrm{Eu}^{2+}$ imaging

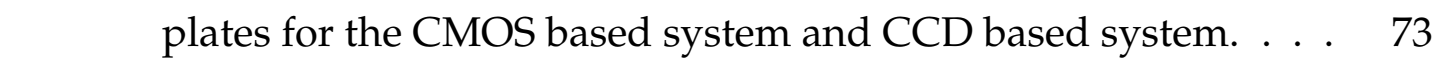

4.9 DQE for $\mathrm{CsBr}: \mathrm{Eu}^{2+}$ and $\mathrm{BaFBr}: \mathrm{Eu}^{2+}$ imaging plates for the CMOS based system and CCD based system . . . . . . . . . . . 75

4.10 X-ray images taken using the prototype imaging system, as explained in the text . . . . . . . . . . . . . . . 77

$5.1 \quad$ PSL active site $\ldots \ldots \ldots \ldots$. . . . . . . . . . . . . . . 82

5.2 As-made CsBr:Eu ${ }^{2+}$ imaging plate and an X-ray image of an IC chip using as-made imaging plate . . . . . . . . . . 83

$5.3 \quad$ Infrared spectrum for as-made $\mathrm{CsBr}: \mathrm{Eu}^{2+}$ imaging plate . . . . . 84

5.4 Optical appearance of $\mathrm{CsBr}: \mathrm{Eu}^{2+}$ imaging plates for various hydration times . . . . . . . . . . . . . . . . 86

$5.5 \quad$ UV-Vis extinction spectra for imaging plates hydrated for various times as shown and transmission coefficient versus hydration time at $633 \mathrm{~nm}$ and $440 \mathrm{~nm} \ldots \ldots \ldots$. . . . . . . . . 87

5.6 SEM images of CsBr:Eu ${ }^{2+}$ imaging plates hydrated for times shown 88 
5.7 Infrared spectra of $\mathrm{CsBr}: \mathrm{Eu}^{2+}$ imaging plates hydrated for various

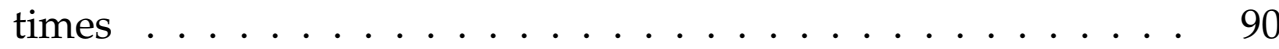

5.8 Photoluminescence emission and excitation (440 $\mathrm{nm}$ emission) spectra for CsBr:Eu ${ }^{2+}$ imaging plates hydrated for various times 91

5.9 Conversion efficiency and stimulation energy for $\mathrm{CsBr}: \mathrm{Eu}^{2+}$ imaging plates as a function of hydration time . . . . . . . . . . 92

5.10 Optical and X-ray modulation transfer functions for the camera system and various imaging plates as indicated. . . . . . . . . . 93

5.11 Images of high spatial frequencies of the lead test grid for pressed imaging plate and commercial needle imaging plate . . . . . . . 94

5.12 Images of $\mathrm{CsBr}: \mathrm{Eu}^{2+}$ imaging plates prepared by hydrating as a powder in room air for times shown . . . . . . . . . . . . 95

5.13 UV-Vis extinction spectra for imaging plates prepared by hydrating as a powder in room air for the times shown and transmission coefficient versus hydration time at 633 and $440 \mathrm{~nm}$. . . . . . 95

5.14 Infrared spectra for imaging plates hydrated in room air as a powder for times shown $\ldots \ldots \ldots$. . . . . . . . . . 96

5.15 PL emission and excitation spectra for imaging plates hydrated in room air as a powder for the times shown $\ldots . . . . . . .997$

$5.16 \mathrm{CE}$ and SE for imaging plates hydrated as a powder in room air as a function of hydration time $\ldots \ldots \ldots$. . . . . . . . 98

5.17 Images of CsBr:Eu ${ }^{2+}$ imaging plates hydrated as a powder in saturated air for times shown . . . . . . . . . . . . . . 98

$5.18 \mathrm{UV}$-Vis extinction spectra for imaging plate hydrated as a powder in saturated air for the times shown and transmission versus hydration time at 633 and $440 \mathrm{~nm} \ldots \ldots$. . . . . . . . 99

5.19 Infrared spectra for imaging plates hydrated in saturated air as a powder for times shown . . . . . . . . . . . . . . . . 100 
5.20 Photoluminescence emission and excitation spectra for imaging

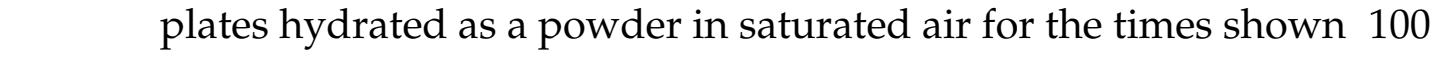

5.21 CE and SE for imaging plates hydrated as a powder in saturated air as a function of hydration time . . . . . . . . . . . . . . 101

5.22 Optical appearance of $\mathrm{CsBr}: \mathrm{Eu}^{2+}$ imaging plates annealed at temperatures shown . . . . . . . . . . . . . . . . 102

5.23 UV-Vis extinction spectra for imaging plates annealed at the temperatures shown and transmission at 633 and $440 \mathrm{~nm}$ as a function of annealing temperature . . . . . . . . . . . 103

5.24 Infrared spectra for $\mathrm{CsBr}: \mathrm{Eu}^{2+}$ imaging plates annealed at the temperatures shown $\ldots \ldots \ldots$. . . . . . . . . . 104

5.25 PSL intensity measured using the camera system and calculated

CE for CsBr:Eu ${ }^{2+}$ imaging plates as a function of annealing tem-

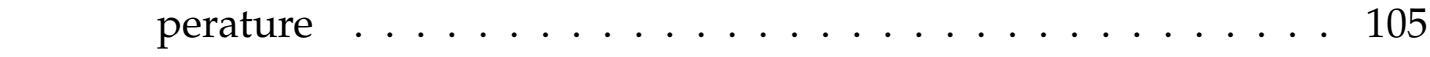

5.26 Electron paramagnetic resonance spectra measured at room temperature for $\mathrm{CsBr}: \mathrm{Eu}^{2+}$ imaging plates annealed at the temperatures shown . . . . . . . . . . . . . . . . . . 107

5.27 Room temperature $9.52 \mathrm{GHz}$ EPR spectrum of a pressed imaging plate annealed at $150{ }^{\circ} \mathrm{C}$ compared to simulated spectra characteristic of the active PSL site . . . . . . . . . . . . . . . . . 109

5.28 Optical appearance of $\mathrm{CsBr}: \mathrm{Eu}^{2+}$ imaging plates hydrated in air at the temperatures shown . . . . . . . . . . . . . . . 111

5.29 UV-Vis extinction spectra for $\mathrm{CsBr}: \mathrm{Eu}^{2+}$ imaging plates hydrated in room air for the temperatures shown, and transmission versus hydration temperature at $633 \mathrm{~nm}$ and $440 \mathrm{~nm} \ldots \ldots . . . . . .111$

5.30 IR spectra for CsBr:Eu ${ }^{2+}$ imaging plates in room air at the temperatures shown . . . . . . . . . . . . . . . . 112

5.31 PL emission and excitation spectra of $\mathrm{CsBr} \mathrm{Eu}^{2+}$ imaging plates hydrated in room air at the temperatures shown . . . . . . . . . 113 
5.32 CE and SE for CsBr:Eu ${ }^{2+}$ imaging plates hydrated as a powder in room air as a function of hydration temperature . . . . . . . . 114

5.33 Optical appearance of imaging plates made by hydrating the powder in saturated air at the temperatures shown $\ldots . . . .115$

5.34 UV-Vis extinction spectra for imaging plates made by hydrating the powder in saturated air at the temperatures shown, and transmission at $633 \mathrm{~nm}$ and $440 \mathrm{~nm}$ as a function of hydration temperature ....................... 115

5.35 IR spectra for imaging plates made by hydrating in saturated air at the temperatures shown $\ldots \ldots \ldots \ldots$. . . . . . . . 116

5.36 PL emission and spectra for imaging plates made by hydrating

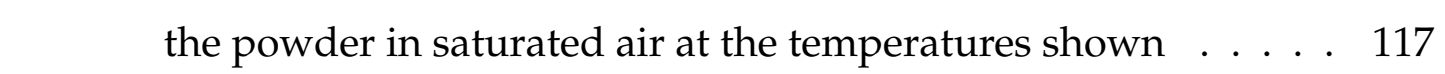

$5.37 \mathrm{CE}$ and SE as a function of annealing temperature for $\mathrm{CsBr}: \mathrm{Eu}^{2+}$ imaging plates hydrated as a powder in saturated air as a function of hydration temperature . . . . . . . . . . . . . 117

5.38 Optical appearance of pre-sintered $\mathrm{CsBr} \mathrm{Eu}^{2+}$ imaging plates hydrated in saturated air for the times shown . . . . . . . . . . . 118

5.39 UV-Vis extinction spectra for pre-sintered imaging plates hydrated in saturated air for the times shown, and transmission at $633 \mathrm{~nm}$ and $440 \mathrm{~nm}$ as a function of hydration time $\ldots . . . . .119$

5.40 IR spectra for pre-sintered $\mathrm{CsBr}: \mathrm{Eu}^{2+}$ imaging plates hydrated in saturated air for the times shown . . . . . . . . . . . . . . . 120

5.41 PL emission and excitation spectra for pre-sintered CsBr:Eu ${ }^{2+}$ imaging plates hydrated in saturated air for the times shown . . 121

$5.42 \mathrm{CE}$ and SE for pre-sintered imaging plates hydrated in saturated air for the times shown $\ldots$. . . . . . . . . . . . . . . . . . 121

5.43 Optical and X-ray MTFs for the camera system and various presintered $\mathrm{CsBr}: \mathrm{Eu}^{2+}$ imaging plates . . . . . . . . . . . 122 
5.44 Representation of PSL active site, $\mathrm{Eu}^{2+}-\mathrm{Cs}_{\mathrm{v}}$ pair, and $\mathrm{Eu}^{2+}-\mathrm{Cs}_{\mathrm{V}}$ agglomerate. The squares represent the unit cell, with each corner containing a $\mathrm{Br}^{-}$ion. . . . . . . . . . . . . . . . . . . . . 124

5.45 Light scattering schematic for transparent and needle imaging plate.

5.46 Estimated MTF at low frequencies for transparent and needle imaging plates . . . . . . . . . . . . . . . . . . . . . . 129

5.47 X-ray images taken with hydrated pressed $\mathrm{CsBr}^{\mathrm{E}} \mathrm{Eu}^{2+}$ imaging plates . . . . . . . . . . . . . . . . . . . 132

6.1 Dual-energy imaging examples . . . . . . . . . . . . . 137

6.2 The basis material plane . . . . . . . . . . . . . . . . 140

6.3 Vector addition of intervening materials . . . . . . . . . . . . 142

6.4 Displacement geometry and simulated high energy, low energy and cancelled X-ray images . . . . . . . . . . . . . . . . . 143

6.5 Material displacement vectors . . . . . . . . . . . . . . . . . . 144

6.6 Schematic showing dual-layered imaging plate for single-shot, single read-out dual-energy imaging . . . . . . . . . . . . . 145

6.7 Incident and absorbed X-ray energy spectra for $70 \mathrm{keV}$ and 150 keV poly-energetic X-ray beams . . . . . . . . . . . . . . . . . 146

6.8 PSL from possible colour dopants as compared to europium doped materials . . . . . . . . . . . . . . . . . . . 148

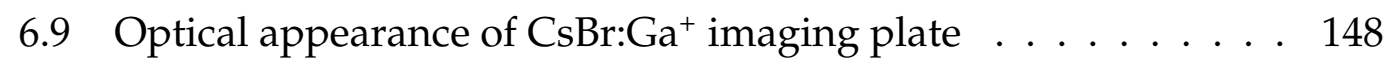

6.10 Optical appearance and X-ray image of IC chip of as-made CsBr:In ${ }^{+}$imaging plate . . . . . . . . . . . . . . . . . . . . 149

6.11 Schematic of target and, low energy, high energy and processed image taken with a dual-layered $\mathrm{CsBr}: \mathrm{Eu}^{2+}$ imaging plate . . . . 151

6.12 Low energy, high energy, and processed X-ray images taken with a dual-layered CsBr imaging plate consisting of an indium doped top layer and europium doped bottom layer 
6.13 PSL of undoped and indium doped CsBr imaging plates . . . . 154

7.1 Neutron capture cross-sections as a function of atomic number . 158

7.2 Schematic of neutron conversion and PSL in storage phosphors 159

7.3 Cut away diagram of OPAL reflector vessel . . . . . . . . . . . 160

$7.4 \quad$ Neutron and X-ray PSL intensity scaled with thickness for imag-

ing plates doped with various europium concentrations, and

fixed europium concentration with various additional neutron converters ........................ 165

7.5 UV-Vis absorbance spectra for a $0.5 \%$ Eu-only doped imaging plate and a $0.2 \% \mathrm{Eu}^{2+}$ doped imaging plate with $1 \%{ }^{6} \mathrm{Li}$ as a neutron converter . . . . . . . . . . . . . . . . . . 168

7.6 Infrared absorbance spectra for a $0.5 \%$ Eu-only doped imaging

\begin{tabular}{|c|}
\hline plate and a $0.2 \mathrm{~mol}^{\%} \mathrm{Eu}^{2+}$ imaging plate with $1 \%{ }^{6} \mathrm{Li}$ as a neutron \\
\hline
\end{tabular}
converter . . . . . . . . . . . . . . . . . . . 169

7.7 X-ray PSL intensities for as-made, annealed and hydrated imaging plates for all dopant types . . . . . . . . . . . . . . . 171

7.8 Neutron and X-ray PSL intensity scaled with thickness for annealed imaging plates doped with various europium concentra\begin{tabular}{|c|}
\hline tions, and fixed europium concentration with various additional \\
\hline
\end{tabular} neutron converters . . . . . . . . . . . . . . . 172

7.9 Corrected neutron PSL intensity for imaging plates doped with various europium concentrations, and fixed europium concentration with various additional neutron converters . . . . . . . . 173

7.10 Optical, X-ray and neutron images of a pen tip taken with the 1 $\%{ }^{6} \mathrm{Li}$ imaging plate . . . . . . . . . . . . . . . . . . . 174 


\section{List of Tables}

2.1 Table of common thermal neutron capture reactions . . . . . 15

$3.1 \quad$ Typical stimulation energies and conversion efficiencies for various storage phosphor materials . . . . . . . . . . . . . . 32

3.2 Comparison of literature CE and SE values to those determined by the integrating sphere method . . . . . . . . . . . . . 44

4.1 dcraw commands for converting Canon CR2 files to a TIFF with raw pixel values . . . . . . . . . . . . . . . . . . 67

4.2 Percentage absorption in $\mathrm{BaFBr}$ and $\mathrm{CsBr}$ for various energies . $\quad 76$

5.1 Line positions for measured IR spectra (as-made imaging plate) compared to those for $\mathrm{SrBr}_{2} \cdot 1 \mathrm{H}_{2} \mathrm{O} \ldots \ldots \ldots \ldots \ldots$

5.2 Spin Hamiltonian parameters used to simulate X band EPR spectra 108

5.3 Best results for each production method, where best is defined as the imaging plate with the highest conversion efficiency . . . 130

6.1 Table of basis material parameters used in dual-energy algorithms 152

7.1 Thermal neutron capture reactions $\ldots \ldots \ldots \ldots \ldots$

7.2 Composition and thickness for CsBr imaging plates doped with various $\mathrm{Eu}^{2+}$ concentrations and no additional neutron converter for the first visit . . . . . . . . . . . . . . . . . . . . 164 
7.3 Composition and thickness for $\mathrm{CsBr}: \mathrm{Eu}^{2+}$ imaging plates doped with $0.2 \mathrm{~mol} \% \mathrm{Eu}$ and various neutron converter materials for the first visit . . . . . . . . . . . . . . . . . . . . 166

7.4 Composition, and thickness for europium only doped imaging plates and imaging plates doped with $0.2 \mathrm{~mol} \% \mathrm{Eu}$ and various neutron converter materials for the second visit. . . . . . . . . . 167 


\section{Chapter 1}

\section{Introduction}

The use of radiation for imaging purposes, particularly imaging the structure of the human body, was evident soon after Röntgen discovered X-rays in 1895. Since then radiation imaging has been used for a wide range of applications such as medical diagnosis, non-destructive testing and security inspections. Radiation is classified into ionising or non-ionising forms; the radiation is ionising if the photons have sufficient energy to remove an electron from an orbital of a free atom (that is, ionise an atom), or equivalently in a solid, to promote an electron from the valence to the conduction band. This thesis is solely concerned with imaging based on ionising radiation. Ionising radiation can take many forms relevant to imaging, including electromagnetic waves, such as X-rays and gamma rays, charged particles (alpha and beta particles), or neutral particles such as neutrons. X-rays and thermal neutrons are the main forms of ionising radiation of interest in this thesis.

The interaction of ionising radiation with matter depends on the type of radiation. X-rays (and gamma rays) interact with matter via the photoelectric effect, Compton scattering and pair production. At energies used for imaging (20$150 \mathrm{keV})$, the photoelectric effect is dominant; this is when an incoming X-ray is absorbed by an atom or ion and it causes an inner electron to be ejected. The attenuation of X-rays in matter is dependent on both the energy of the incident wave and the actual or effective atomic number $(Z)$ of the material. Figure 


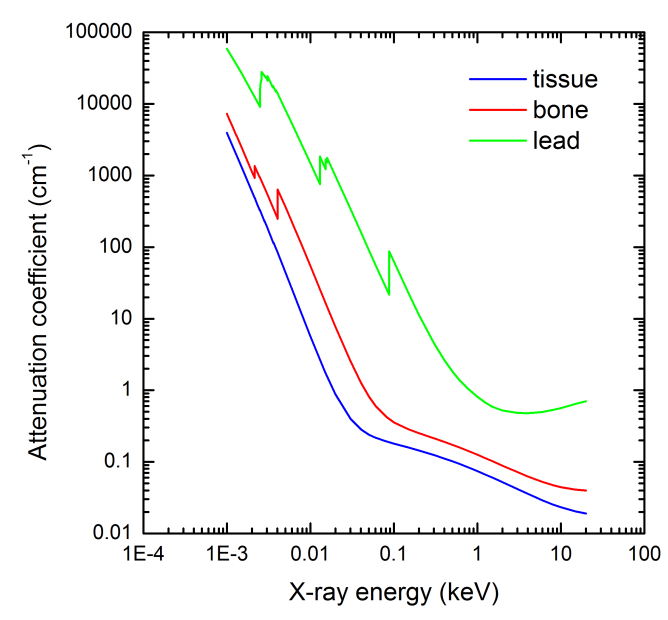

Figure 1.1: Attenuation coefficient versus energy for tissue (blue), bone (red) and lead (green) (data from National Institute of Standards and Technology [1]).

1.1 shows typical attenuation curves for tissue $\left(Z_{\text {eff }}=7.4\right)$, bone $\left(Z_{\text {eff }}=14\right)$ and lead $(Z=82)$. The higher the atomic number the greater the attenuation, and in general, the higher the energy of the incident X-ray the lower the attenuation (except at high energies due to pair production). The difference in attenuation coefficient between bone and tissue (figure 1.1) is the basis for medical imaging; the high attenuation coefficient for lead explains its use in radiation shielding. Neutrons interact with matter by elastic or inelastic scattering and capture reactions; in the latter a neutron is captured and the nucleus undergoes a reaction, emitting alpha or beta particles and/or gamma rays. Capture reactions are the main attenuating process for thermal neutrons (hydrogen is an exception where scattering is dominant). The attenuation of thermal neutrons through matter is roughly constant with atomic number (figure 1.2), but with some elements (or more precisely particular isotopes) such as hydrogen, boron, gadolinium and europium having unusually high attenuation coefficients. Imaging with neutrons is generally complementary to X-rays in that objects with a hydrogen-rich composition (for example water, tissue and plastics) attenuate the transmitted neutron beam more strongly than materials such as metals or bone. For X-rays, the converse is true.

Imaging with X-rays and neutrons cannot be done simply with readily available 


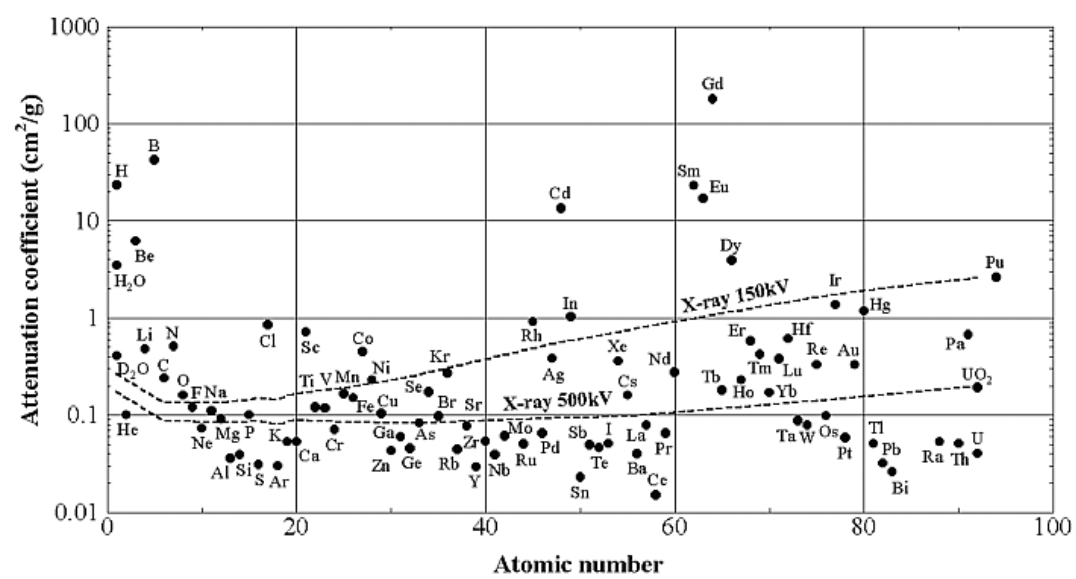

Figure 1.2: Thermal neutron attenuation coefficient for most elements [2].

optics (mirrors and lenses), as is used for visible electromagnetic radiation. The refractive index for a material at X-ray energies is essentially one, and the radiation simply passes straight through without significant refraction or reflection. The nature of neutron interactions also means optics cannot be used. Instead shadow imaging techniques are used where the attenuation of an X-ray or neutron beam through an object is recorded in a projected image on a medium (figure 1.3).

X-ray and neutron imaging have a wide range of applications, which includes medical, dental and veterinarian radiography, security inspections (airport baggage screening), non-destructive testing applications such as pipe inspection, and production line quality assurance (for example, meat to fat ratios). Medical applications for neutron imaging are much less common as neutron sources are

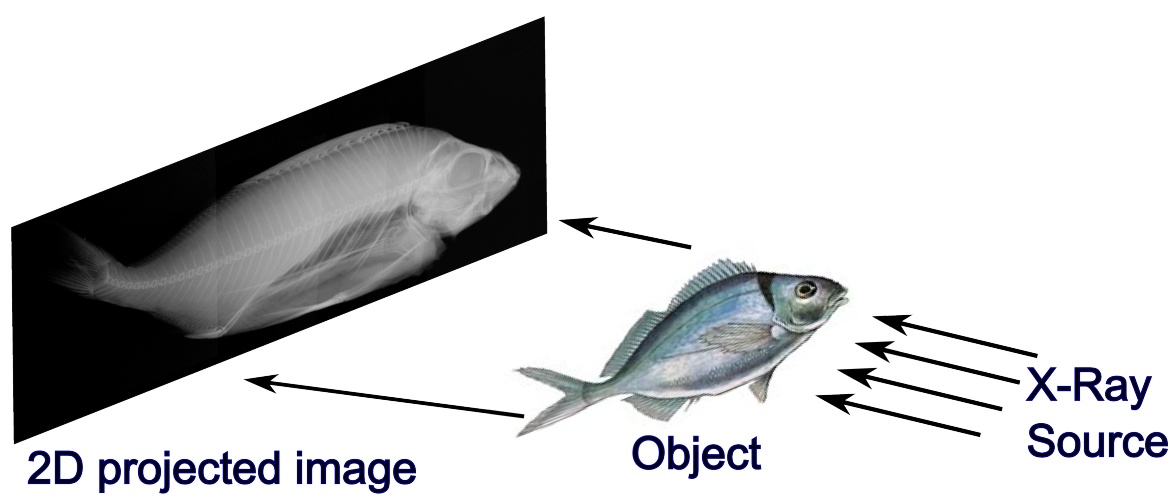

Figure 1.3: Demonstration of a projection X-ray image. 
potentially hazardous, difficult to obtain and require large amounts of shielding. Neutrons can be used for non-imaging medical applications such as boron neutron capture therapy [3] for the treatment of cancerous tumours. However, neutron imaging is mainly used for non-destructive testing, and industrial and security applications.

Historically X-ray images were recorded using photographic film which consists of a thin light sensitive emulsion layer on a plastic backing. The light sensitive material is a silver halide, and the $\mathrm{X}$-rays incident on the film cause the silver ions to convert to silver metal. Developing and fixing of the film is required to enhance and preserve the image. The resolution and sensitivity of the film (and the image) is determined by the grain size of the silver halide. Photographic film is not particularly sensitive to X-rays but is very sensitive to visible light. To take advantage of this, intensifying screens consisting of materials such as calcium tungstate or rare-earth phosphors are used to convert the X-ray photons to visible photons (one X-ray photon creates many visible photons). The intensifying screens increase the sensitivity of the system but decrease the resolution due to light scattering within the powdered screen. An alternative to photographic film, in the form of storage phosphor imaging plates, was introduced in the early 1980's by Sonoda et al. [4]; a technique known commercially as computed radiography (CR). The first imaging plates were based on a powdered storage phosphor material $\left(\mathrm{BaFBr}: \mathrm{Eu}^{2+}\right)$ embedded in an organic binder placed on a support film. In a storage phosphor imaging plate, incident $X$-rays generate equal numbers of electrons and holes, some of which are trapped within the crystal lattice, creating a latent image. To read out the image, a focussed laser beam is raster scanned across the plate, stimulating recombination of electrons and holes which results in recombination luminescence. This luminescence is recorded, and the intensity of emission across the plate is the image of the object under investigation. Imaging plates have many advantages over film such as re-usability, a digital image suited for 


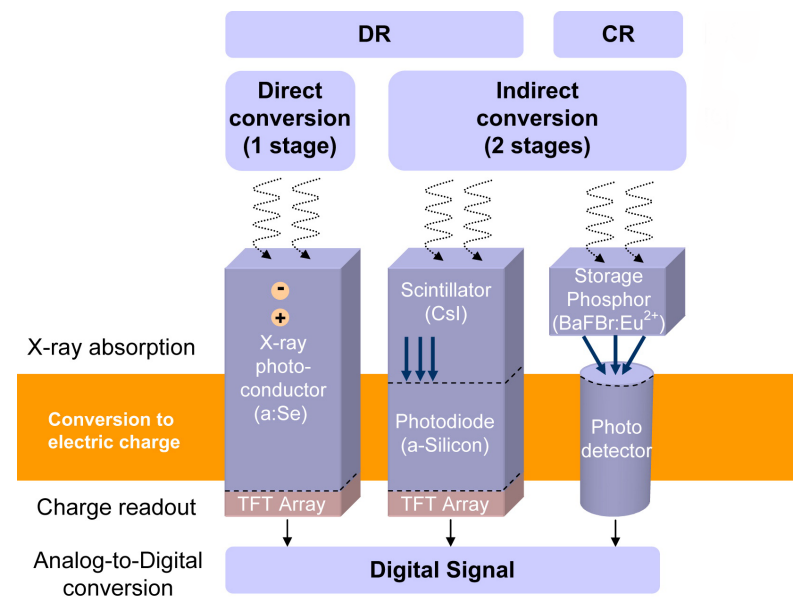

Figure 1.4: General description model for digital X-ray technologies [6].

image processing, larger dynamic range, no chemical processing and a high sensitivity. However, they suffer from a lower spatial resolution than film due to light scattering from the phosphor grains, as explained later. Newer storage phosphor imaging plates in the form of needle-structured $\mathrm{CsBr}: \mathrm{Eu}^{2+}$ have been developed, in which the needles are orientated perpendicular to the plate. These needle imaging plates show a higher resolution than the powdered materials due to the needles partially guiding the stimulation light and thus reducing the light scattering effects. A more detailed discussion on storage phosphor imaging plates, with an emphasis on $\mathrm{CsBr}: \mathrm{Eu}^{2+}$ will be given in chapter 2 Other forms of digital imaging (digital radiography, DR) have since been developed which compete with storage phosphor imaging plates [5, 6]. The first technique uses a phosphor layer, commonly CsI or $\mathrm{Gd}_{2} \mathrm{O}_{2} \mathrm{~S}$, to convert the X-ray photons to visible photons (see figure 1.4). These visible photons are converted to an electronic signal using a photodiode layer (amorphous silicon) and the light intensity is recorded using a thin film transistor (TFT) array. This technique is known as indirect digital radiography due to the phosphor layer required to convert the X-ray photons into visible photons required for detection.

A newer technique, "direct digital radiography", uses an amorphous selenium layer which directly generates electron hole pairs [7]. The electrons and holes are charge separated using a strong electric field and the resulting current flow 


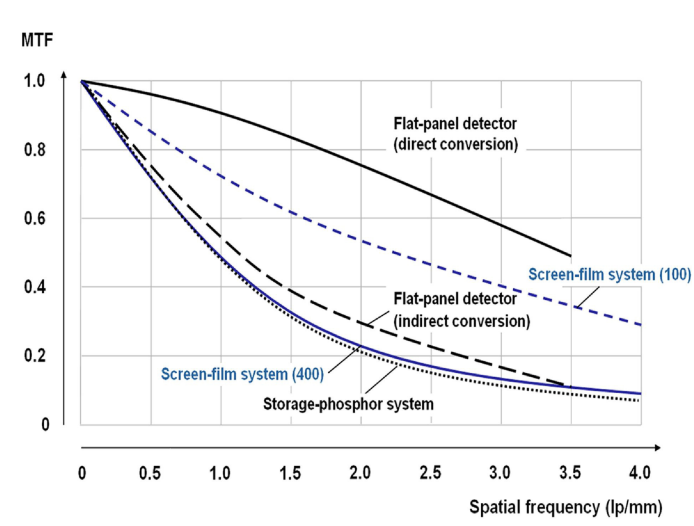

(a)

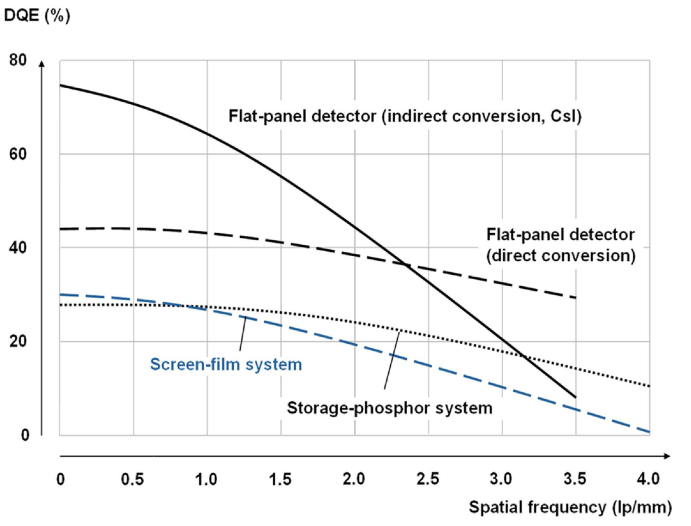

(b)

Figure 1.5: Comparison of storage phosphors, film and digital imaging systems in terms of (a) spatial resolution (modulation transfer function) and (b) sensitivity (detective quantum efficiency) [9]. The (100) and (400) for screen-film systems refer to the speed class of the film.

is read out using thin-film transistors or diodes. This is a purely electronic technique, as opposed to the previous mentioned optical techniques, and shows an improved spatial resolution as the electrons are guided on straight paths through the detector by the electric field. However, these DR systems are both expensive and fragile.

Another direct system is based on the Medipix detectors developed at CERN [8], which consist of a semiconductor detector layer bump-bonded to a CMOS pixel read-out chip. These detectors are unique in that on-pixel electronic circuitry means they individually count the X-ray photons and can provide crude spectral analysis based on the incident $X$-ray energy using pulse height discrimination. However, the imaging plates are relatively small in size (approximately $14 \times 14 \mathrm{~mm}^{2}$ ) and expensive due to the high amount of circuitry required.

Computed and digital radiography systems can also be used for neutron imaging. This requires a neutron convertor element such as gadolinium, boron or lithium to convert the neutrons to gamma rays or alpha particles which can then be detected by the digital imaging system. Current commercial neutron storage phosphor imaging plates consist of $\mathrm{BaFBr}: \mathrm{Eu}^{2+} \mathrm{co}-\mathrm{d}$ oped with gadolinium. The current storage phosphor imaging plates cannot compete with the newer digital radiography systems in terms of their sensitivity and spatial resolution, 
but are much more cost-effective. The resolution is measured in terms of a modulation transfer function, and as shown in figure 1.5(a), is significantly broader for the direct conversion system. The sensitivity of an imaging system is measured by its detective quantum efficiency (figure 1.5(b)), which shows a much lower value for storage phosphor systems compared to digital systems. Currently, the most widely used storage phosphor imaging plates are based on $\mathrm{BaFBr}: \mathrm{Eu}^{2+}$, and read-out occurs by a raster scanned focussed laser beam. The read-out laser light is scattered from the phosphor grain boundaries, resulting in a read-out area which is much larger than the laser spot, and hence a low spatial resolution. However, a transparent storage phosphor would improve both the resolution and sensitivity, as a thicker layer could be made without the resolution problems caused by scattering. Transparent glass ceramic storage phosphor materials have been developed which show high resolution [10], but lack the sensitivity for imaging applications.

$\mathrm{CsBr}: \mathrm{Eu}^{2+}$ needle structured imaging plates have been found to be high performing detectors for computed radiography applications, with a better sensitivity and spatial resolution than $\mathrm{BaFBr}: \mathrm{Eu}^{2+}[11]$. Currently these imaging plates are grown by a thermal evaporation technique, which requires relatively expensive equipment to obtain uniform needle structures over large areas. Consequently, a CsBr:Eu ${ }^{2+}$ needle imaging plate (NIP) currently costs around $\$ 6000$, as compared to $\$ 300$ for a $\mathrm{BaFBr}: \mathrm{Eu}^{2+}$ plate of similar size.

Simple ceramic $\mathrm{CsBr}: \mathrm{Eu}^{2+}$ imaging plates made by a similar process to that for $\mathrm{BaFBr}: \mathrm{Eu}^{2+}$ would be more cost-effective than NIPs, but would offer no particular advantage as the same resolution-limiting light scattering would occur. However, there is an important structural difference between $\mathrm{CsBr}$ and $\mathrm{BaFBr} ; \mathrm{CsBr}$ is cubic, and therefore has an isotropic refractive index. This means that a compressed polycrystalline pellet, similar to the $\mathrm{KBr}$ pellets commonly used in IR spectroscopy, may be a transparent (with low optical scattering), high resolution $\mathrm{X}$-ray imaging material. This idea was encouraged by the ob- 
servation [12] that small polycrystalline $\mathrm{CsBr}: \mathrm{Eu}^{2+}$ samples prepared in a hand press for spectroscopic studies at the DESY synchrotron appeared to be very transparent. Alkali halides, particularly $\mathrm{KBr}$, are widely used in infrared spectroscopy as pressed disc encapsulants. Materials to be studied are ground with $\mathrm{KBr}$ and pressed into a pellet in a hydraulic press. Plastic flow of the $\mathrm{KBr}$ results in a pellet disc, which is transparent in the infrared and visible wavelength range. Is it possible, given the extensive experience with transparent $\mathrm{KBr}$ in IR spectroscopy, to produce a transparent or semi-transparent $\mathrm{CsBr}: \mathrm{Eu}^{2+}$ imaging plate in the form of a pressed disc?

The aims of this thesis were firstly to develop transparent $\mathrm{CsBr}: \mathrm{Eu}^{2+}$ imaging plates for use in high resolution applications such as mammography. This was achieved by cold sintering (pressing) fine powders of $\mathrm{CsBr}$ and $\mathrm{EuBr}_{2}$.

In a second objective, a new cost-effective read-out technique has been developed for reading out storage phosphor plates.

In a third objective, imaging plates with other dopants such as $\mathrm{In}^{+}, \mathrm{Sm}^{2+}$ and $\mathrm{Ga}^{+}$ have been developed for use in dual energy applications. Dual energy imaging is fast becoming an important technique as it gives chemical information on object composition.

Finally, in a fourth objective, neutron imaging plates consisting of CsBr:Eu ${ }^{2+}$ doped with lithium, boron or gadolinium compounds were investigated for their use in neutron imaging.

This thesis is structured as follows. Chapter 2 contains relevant background information, and a brief review of literature on $\mathrm{CsBr}$ imaging plates. Chapter 3 describes the experimental methods used. Chapter 4 introduces a new technique for capturing $X$-ray images using a digital camera. Chapter 5 contains the experimental results for $\mathrm{CsBr}: \mathrm{Eu}^{2+}$ imaging plates, chapter 6 their use in dual energy imaging and chapter 7 their use for neutron imaging. 


\section{Chapter 2}

\section{Background}

In this chapter a review of imaging with ionising radiation using storage phosphor imaging plates is presented. Firstly, ionising radiation in the form of $\mathrm{X}$-rays and neutrons is discussed, including their interaction with matter. The process of light emission of phosphors (storage and other) in the form of photoluminescence and photostimulated luminescence is explained. The structure and performance of current storage phosphor imaging plates is discussed, with an emphasis on those based on $\mathrm{CsBr}: \mathrm{Eu}^{2+}$.

\subsection{Ionising radiation}

\subsubsection{X-rays}

X-rays are high energy electromagnetic waves with wavelengths in the range $0.1-10 \mathrm{~nm}$, and are generated by electronic transitions between energy levels in an excited atom. In an X-ray generator, electrons produced by a hot cathode filament are accelerated towards a metal anode target, where the electrons lose their energy through two processes. In the first, the electrons collide with the bound electrons of the target atoms causing an inner shell electron to be ejected. An electron from a higher orbital shell fills the vacancy causing an X-ray photon to be emitted with an energy equal to the energy difference of the 


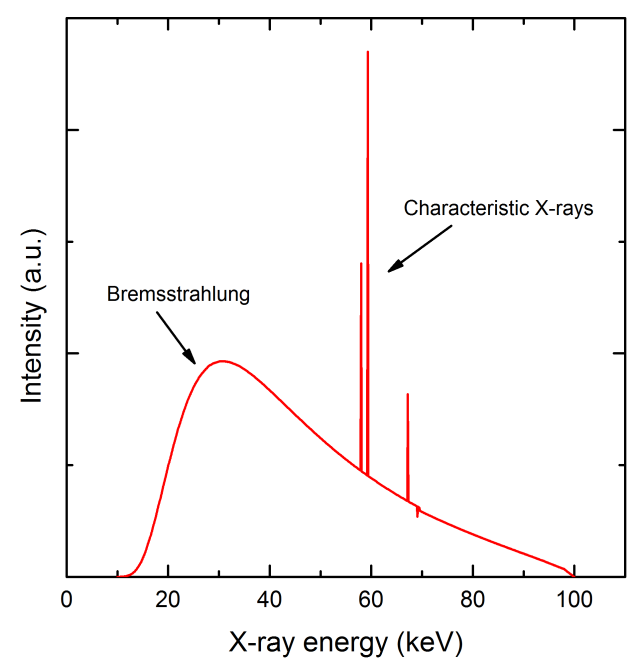

Figure 2.1: Example of a tungsten $X$-ray spectrum showing characteristic $X$-rays and bremsstrahlung [13].

two shells. The energy of these X-rays are characteristic of the target material and appear as discrete sharp lines in the X-ray spectrum (see figure 2.1). The second process is known as bremsstrahlung ("braking radiation"), and occurs when the electrons incident on the target are subject to the electrostatic forces with the target atom's electronic shells, causing the electrons to decelerate and emit X-rays. This results in a continuous energy spectrum of X-rays, as can be seen in figure 2.1. on which the characteristic target peaks are superimposed. Due to the high energy of X-rays they are easily able to pass through matter. Attenuation of the X-ray beam intensity occurs due to interactions between X-rays and the atoms in a material, and is given by a Beer-Lambert type law,

$$
I=I_{0} \exp \left(-\mu_{\text {tot }} z\right)
$$

where $I$ is the transmitted intensity, $I_{0}$ is the initial intensity, $z$ the material thickness and $\mu_{\text {tot }}$ the total attenuation coefficient. Three main effects cause the attenuation of $\mathrm{X}$-rays through matter; the photoelectric effect, Compton scattering and pair production. The total attenuation coefficient is a linear combination of the three separate processes [14], that is,

$$
\mu_{\mathrm{tot}}=\mu_{\mathrm{PE}}+\mu_{\mathrm{CS}}+\mu_{\mathrm{PP}},
$$



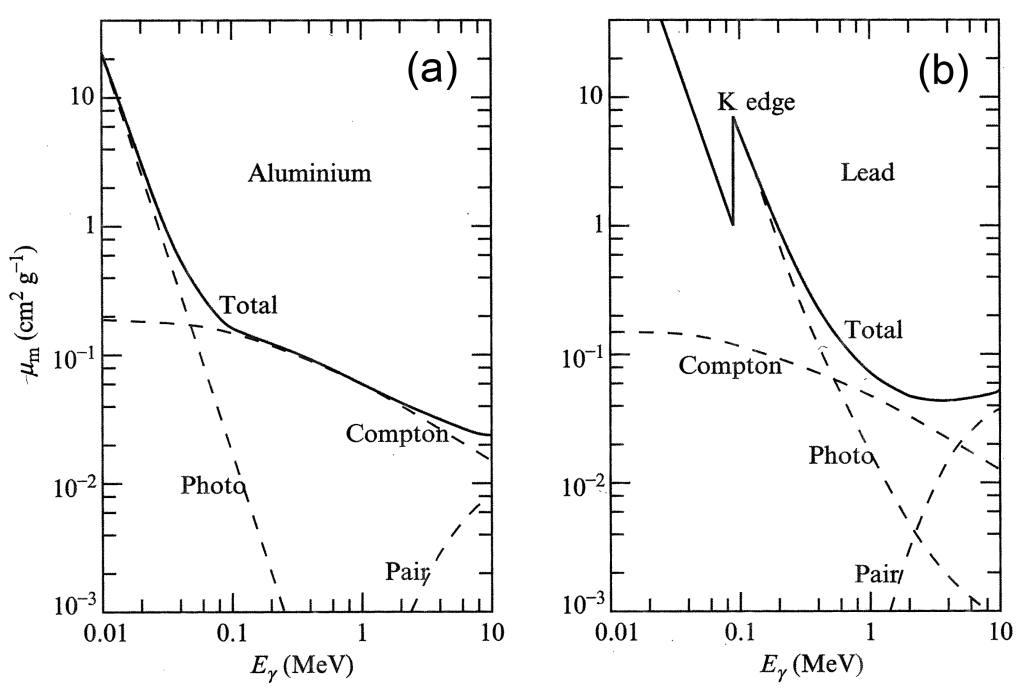

Figure 2.2: Mass attenuation coefficients (solid lines) as a function of X-ray energy for (a) aluminium and (b) lead showing contributions from the photoelectric effect, Compton scattering and pair production (dashed lines) [14]. The region of interest for X-ray imaging is approximately $15-150 \mathrm{keV}$.

where $\mu_{\mathrm{PE}}, \mu_{\mathrm{CS}}$ and $\mu_{\mathrm{PP}}$ are the photoelectric, Compton and pair production attenuation coefficients respectively. The contribution of each of these processes to the mass attenuation coefficient (attenuation coefficient divided by material density) is shown for aluminium and lead in figure 2.2. At energies used for imaging (approximately 15 - $150 \mathrm{keV}$ ), the photoelectric effect is the dominant process with a small contribution from Compton scattering. Pair production only occurs at much higher energies since a minimum energy of $2 m c^{2} \sim 1024$ $\mathrm{keV}$ is required to produce an electron positron pair. The two main process are now discussed.

\section{Photoelectric effect}

Photoelectric absorption occurs when an X-ray interacts with an atom by transferring all its energy to an orbital electron (usually from an inner shell) causing the electron to be released from the atom (figure 2.3). The emerging "photoelectron" has kinetic energy equal to the difference between the initial X-ray photon energy minus the binding energy of the electron. The vacancy in the shell can be filled by an electron in a higher orbital with either the release of a 

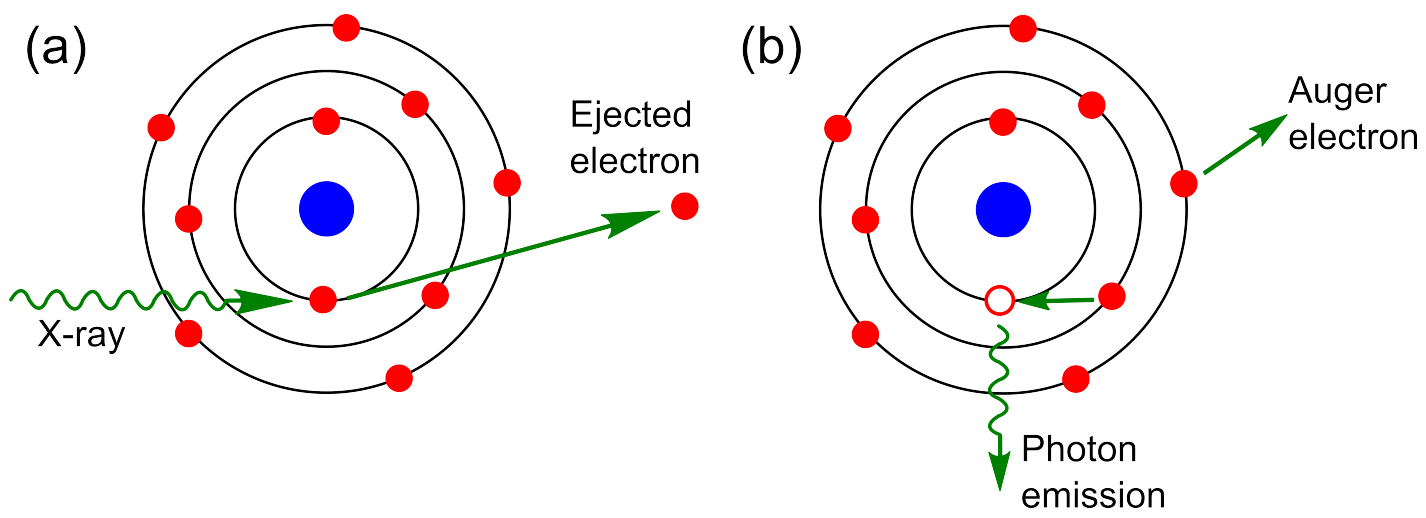

Figure 2.3: Schematic of photoelectric process. (a) Incident X-ray causes inner orbital electron to be ejected. (b) Vacancy is filled by outer orbital electron with emission of a photon or Auger electron.

photon (X-ray fluorescence) or by transferring its energy and releasing another electron from the same atom (Auger electron).

The photoelectric attenuation coefficient depends on both the atomic number $(Z)$ of the material and the X-ray photon energy $(E)$, and is given approximately by [15],

$$
\mu_{\mathrm{PE}} \propto \frac{Z^{4}}{E^{3}}
$$

Note that this empirical formula ignores the effect of the sharp edges visible in figure 2.2(b) arising from the threshold energies for the K, L, M etc. electron shells; K, L, M are the first three electron shells with $n=1,2$ and 3 respectively.

\section{Compton Scattering}

Compton scattering is an interaction where an incident $\mathrm{X}$-ray photon is inelastically scattered from a free or loosely bound electron, and is shown in figure 2.4. The result is a lower energy photon being scattered by an angle $\theta$ from the incident direction, and the electron recoiling with a kinetic energy dependent on the scattering angle. Energy and momentum are conserved in the collision process and the resulting angular variation for the scattering probability is described in many texts (for example, Lilley [14]).

The probability of Compton scattering does not depend as strongly on atomic number and X-ray energy as the photoelectric effect. Instead of depending on $Z$ 


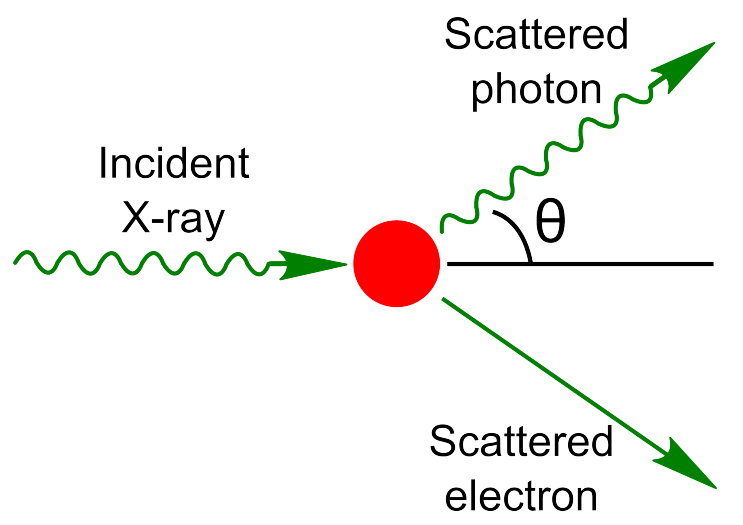

Figure 2.4: Schematic of Compton scattering.

alone, the probability depends on the density of the material multiplied by $Z / A$ ( $A$ is the atomic mass number), which is relatively constant across the periodic table. Therefore, the mass attenuation coefficient is approximately independent of $Z$, but does depend on the X-ray energy. The dependence on energy is given by the Klein-Nishina [15] formula and is a function of the scattering angle. The formula predicts that the mass attenuation coefficient will decrease with photon energy, as is seen in figure 2.2

\subsubsection{Neutrons}

A neutron is a subatomic particle with zero electric charge, meaning it can easily penetrate the electron shells of an atom and directly interact with the nucleus. To generate a free neutron requires liberating it from the forces that hold the nucleus together. Free neutrons are unstable and normally undergo beta decay to form a proton, resulting in a free neutron with a half life of approximately 15 minutes. Free neutrons can be generated by nuclear reactions such as alpha or gamma bombardment of certain heavy nuclei, nuclear fission, or by disintegration of nuclei in high energy nuclear reactions, such as collisions in accelerators or in cosmic radiation.

Neutrons are characterised by their kinetic energy or neutron temperature. This thesis is solely concerned with "thermal neutrons" which are neutrons in thermal equilibrium with their surroundings at room temperature (290 K), 
having a most probably energy equal to $k T=25 \mathrm{meV}$ (average energy $\frac{3}{2} k T=38$ meV). Thermal neutrons are produced by moderating, or slowing down, higher energy neutrons via collisions with various atoms. This occurs in nuclear reactors where the core generates high energy neutrons which are moderated by a surrounding reaction vessel consisting of (normal or heavy) water (see chapter 7).

Neutrons interact with matter through nuclear reactions which depend on both the incident neutron energy and the target nuclei. For thermal neutrons the dominant attenuation processes are elastic scattering and neutron capture reactions.

In inelastic scattering the incident neutron collides with the nucleus of an atom causing the nucleus to recoil and the neutron to be scattered with a lower kinetic energy (similar to figure 2.4). The kinetic energy of the scattered neutron depends on the atomic number of the target nucleus and the angle of scattering; more energy is lost when scattering from low atomic number nuclei. With thermal neutrons, the energy absorbed by the material (less than $25 \mathrm{meV}$ ) is not enough to create the electron-hole pairs required for imaging with storage phosphors (which requires energy greater than the $6-8 \mathrm{eV}$ band gap). Instead it is the second process, neutron capture, which is more important.

Neutron capture occurs when a free neutron is absorbed by a nucleus, creating a heavier isotope of the capture atom. Often this new isotope is unstable and subsequently decays creating lighter atoms along with gamma rays, alpha or beta particles and conversion electrons. Particular isotopes, such as lithium-6, boron-10 and gadolinium-155 or -157 have a high capture cross-section (see figure 1.2), and are therefore effective at capturing neutrons and converting the energy to a useful form. Some capture reactions are shown in table 2.1 For neutrons it is the decay products which are detected, and in imaging with storage phosphors the decay products provide the energy required to create electron-hole pairs. 
Table 2.1: Table of common thermal neutron capture reactions [16]. 1 barn $=$ $10^{-24} \mathrm{~cm}^{2}$, "ce" = conversion electron.

\begin{tabular}{ccc}
\hline Element & Reaction & Cross section (barns) \\
\hline${ }^{3} \mathrm{He}$ & ${ }^{3} \mathrm{He}+{ }^{1} \mathrm{n} \rightarrow{ }^{3} \mathrm{H}+{ }^{1} \mathrm{p}+0.77 \mathrm{MeV}$ & 5333 \\
${ }^{6} \mathrm{Li}$ & ${ }^{6} \mathrm{Li}+{ }^{1} \mathrm{n} \rightarrow{ }^{3} \mathrm{H}+{ }^{4} \mathrm{He}+4.79 \mathrm{MeV}$ & 940 \\
${ }^{10} \mathrm{~B}$ & ${ }^{10} \mathrm{~B}+{ }^{1} \mathrm{n} \rightarrow{ }^{7} \mathrm{Li}+{ }^{4} \mathrm{He}+2.78 \mathrm{MeV}(7 \%)$ & 3837 \\
& $\rightarrow{ }^{7} \mathrm{Li}+{ }^{4} \mathrm{He}+\gamma(93 \%)$ & \\
${ }^{155} \mathrm{Gd}$ & ${ }^{155} \mathrm{Gd}+{ }^{1} \mathrm{n} \rightarrow{ }^{156} \mathrm{Gd}+\gamma+\mathrm{ce}$ s & 61000 \\
${ }^{157} \mathrm{Gd}$ & ${ }^{157} \mathrm{Gd}+{ }^{1} \mathrm{n} \rightarrow{ }^{158} \mathrm{Gd}+\gamma+\mathrm{ce}$ s & 254000 \\
\hline
\end{tabular}

\subsection{Luminescence mechanisms}

Luminescence occurs when electronic states of solid materials are excited by an external energy source and the excitation energy is released as light. Storage phosphors emit light by a phenomenon known as photostimulated luminescence (PSL). To understand PSL, the mechanisms of photoluminescence must first be considered.

\subsubsection{Photoluminescence}

Photoluminescence (PL) occurs when the external energy source in the above definition of luminescence comes from short wavelength light [17]. PL can be split into two main categories, intrinsic and extrinsic luminescence. Intrinsic luminescence is further separated into band to band, cross and exciton luminescence; extrinsic luminescence is divided into localised or unlocalised depending on whether optical excitation and emission occur at a localised centre or if the electrons and holes of the host lattice participate in the luminescence process. In this thesis we deal with extrinsic localised luminescence, where ionic crystals or semiconductors are doped with cationic impurities (such as transition metals or rare earth ions) to create localised luminescence centres.

In localised luminescence, the excitation and emission properties predominantly occur by electric dipole transitions between energy levels of the luminescence centres. The properties of these processes can be described using a 
configuration-coordinate model (figure 2.5(a)) which shows the potential energy curves of the luminescence centre as a function of a configurational coordinate, $q$. This coordinate describes a vibrational mode of the luminescence centre. As a simple example, the symmetrical stretching mode of vibration has the luminescence centre at rest and the surrounding ions move in phase towards and away from the centre. In this case, $q$ is the distance between the luminescence centre and the surrounding ions. The curves in figure 2.5(a) show the ground (g) and excited (e) states of the luminescence centre, and are representative of the $\mathrm{Eu}^{2+}$ ion. The shift in the position of the minimum energy between the ground and excited states is due to the different electronic configurations of the two states, for example, for $\mathrm{Eu}^{2+}$ the ground state is $4 f^{7}$ and the excited state is $5 d^{1}$. This leads to different interionic forces between the $\mathrm{Eu}^{2+}$ ion and its neighbours for the ground and excited states, and therefore the shift in equilibrium position. The vibrational motion is assumed to be harmonic leading to the parabolic shape of the curves, and the energy levels (horizontal lines) are those of a harmonic oscillator, given by,

$$
E=\left(n+\frac{1}{2}\right) h \nu
$$

where $n=0,1,2, .$. and $\nu$ is the oscillator frequency.

The probability of an optical transition between a ground and excited vibrational level is proportional to,

$$
\left\langle\phi_{\mathrm{Eg}}|\mathbf{e} \cdot \mathbf{r}| \phi_{\mathrm{Ee}}\right\rangle\left\langle\phi_{\mathrm{Vg}} \mid \phi_{\mathrm{Ve}}\right\rangle
$$

where $\phi_{\mathrm{E}}$ are the electronic wavefunctions, $\phi_{\mathrm{V}}$ are the vibrational wavefunctions, $\mathbf{e} \cdot \mathbf{r}$ is the electronic dipole operator with $\mathbf{e}$ the charge and $\mathbf{r}$ the position. Examples of the vibrational wavefunctions are shown in figure 2.5(b). The first term in equation 2.5 is independent of vibrations and gives the electronic factor contributing to the intensity of the transition. Transitions are governed by the parity selection rule which dictates whether the transitions are dipole allowed 


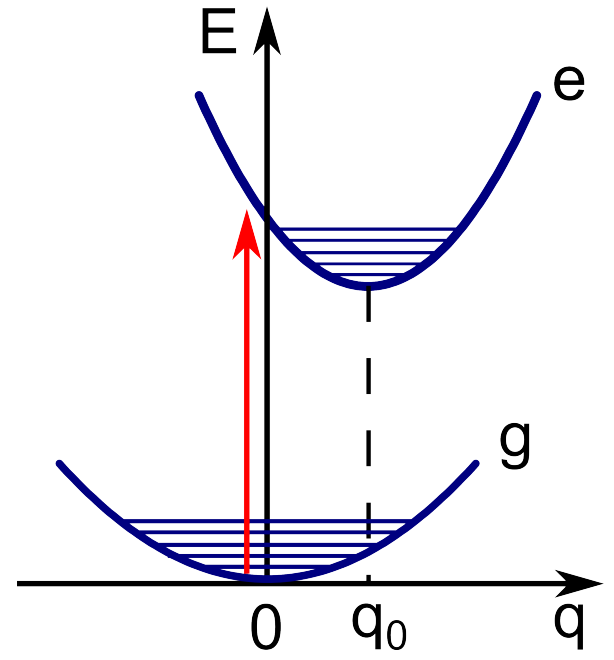

(a)

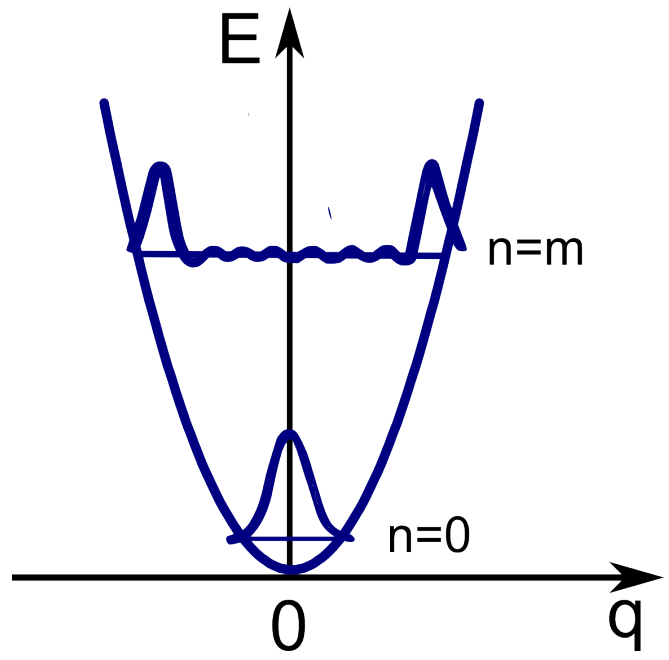

(b)

Figure 2.5: (a) One dimensional configuration-coordinate model for a localised luminescence centre and (b) vibrational wavefunctions for the lowest vibrational level $(n=0)$ and a high vibration level $(n=m)$ [17].

$(\Delta l= \pm 1)$ or forbidden $(\Delta l=0)$. If the luminescence centres are incorporated in crystals the nominally forbidden transitions may become partially allowed due to the parity mixing of state by odd components in the crystal electric field. An example of an allowed transition is a $f \rightarrow d$ shell transition(e.g., $\mathrm{Eu}^{2+}$ or $\left.\mathrm{Ce}^{3+}, 4 f \rightarrow 5 d\right)$ and examples of forbidden transitions are $d \rightarrow d$ (e.g., $\mathrm{Mn}^{2+}$, $\left.3 d^{5} \rightarrow 3 d^{5}\right)$ and $f \rightarrow f\left(\right.$ e.g., $\mathrm{Eu}^{3+}$ or $\left.\mathrm{Sm}^{3+}, 4 f^{n} \rightarrow 4 f^{n}\right)$ shell transitions. The second term in equation 2.5 is the vibrational overlap of the wavefunctions and determines the shape of the excitation or emission band.

An incident UV photon causes a transition from the centre's ground vibrational state to an excited state, which is shown as a vertical transition on figure 2.5(a) At low temperatures the highest intensity transition occurs (at $q=0$ ) from the lowest vibrational level $(n=0)$ to a high vibrational level in the excited state (for example $n=m$ ), as the vibrational wavefunctions have maximum overlap there.

Photoluminescence comes from the relaxation of the centre from the excited to ground state. Due to the initial absorption, the centre is in a high vibrational level of the excited state, and this relaxes to the lowest vibrational level of the excited state by emission of phonons. Emission then occurs as the centre relaxes 
to one of the ground vibrational levels.

Electric dipole allowed transitions (e.g. $5 d \rightarrow 4 f$ ) have broad spectral bands which is due to both the lowest ground vibrational state overlapping with several excited state levels, and at higher temperatures, higher ground vibrational levels may be occupied. Electric dipole forbidden transitions (for example $4 f \rightarrow 4 f$ ) have the minimum of the excited vibrational curve close to $q=0$ and as both vibrational wavefunctions are maximal there the transition is seen as a single sharp line. This transition (excitation and emission) is called the zero phonon transition as no vibrations are involved.

\subsubsection{Photostimulated luminescence}

Photostimulated luminescence (PSL) is a similar process to photoluminescence but with a delayed, stimulated emission. A schematic of the imaging process using storage phosphors is given in figure 2.6. In PSL the ionising radiation incident during irradiation causes electrons to be promoted to the conduction band, leaving behind an equal number of holes; many electrons are promoted as the energy of the $\mathrm{X}$-ray $(\sim 10-200 \mathrm{keV})$ is much higher than the band gap of the host lattice $(\sim 6-8 \mathrm{eV})$. The electrons are trapped at defects in the lattice, typically as F centres (an electron trapped at an anion vacancy), just below the conduction band; the holes are similarly trapped just above the valence band at hole traps. The latent image is stored in the imaging plate as a spatially varying concentration of trapped electron-hole pairs.

In a commercial read-out system, the stimulating light is a focussed laser beam which is raster scanned (using mirrors) across the imaging plate, causing the electrons to be freed from the traps and recombine with holes. The recombination energy is transferred to the luminescence centre (activator ion) and characteristic luminescence is emitted. The luminescence is detected by a photomultiplier tube and converted to a digital signal by analogue-to-digital converter for analysis on a computer. The image is then stored digitally on 


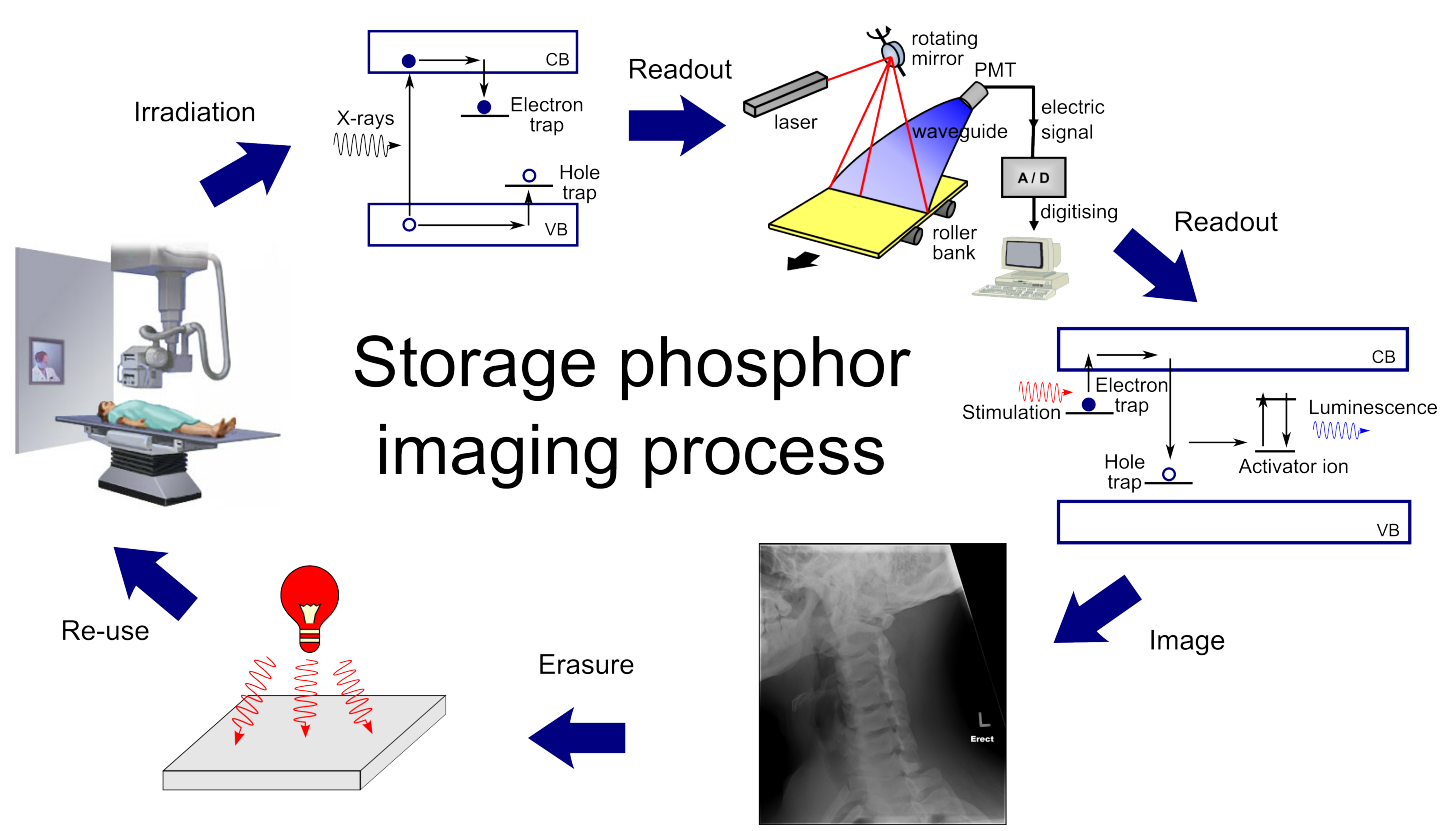

Figure 2.6: Schematic of imaging with storage phosphor imaging plates (combined illustration [18, 19]).

the computer. An alternative read-out technique based on commercial digital cameras is given in chapter 4 .

\subsection{Storage phosphor imaging plates}

\subsubsection{BaFBr imaging plates}

Historically, the family of $\mathrm{BaFX}: \mathrm{Eu}^{2+}(\mathrm{X}=\mathrm{Br}, \mathrm{Cl})$ phosphors were used as intensifying screens for photographic film, but suffered from a high level of afterglow (delayed luminescence after irradiation) which is now known to be due to the PSL process. The first storage phosphor material discovered was based on $\mathrm{BaFBr}: \mathrm{Eu}^{2+}$ and led to the further development of computed radiography and commercialisation by Fuji in the mid 1980s [4].

$\mathrm{BaFBr}: \mathrm{Eu}^{2+}$ is formed by mixing the raw materials $\mathrm{BaF}_{2}$ and $\mathrm{BaBr}_{2}$ and a europium dopant and firing at a temperature just below the melting point to allow mixing by diffusion - a sintering process. The product is milled to a powder, embedded in an organic binder and spread on a support film to make an imaging plate. The structure of the $\mathrm{BaFBr}$ host lattice is a tetragonal matlockite $\mathrm{PbFCl}$ 


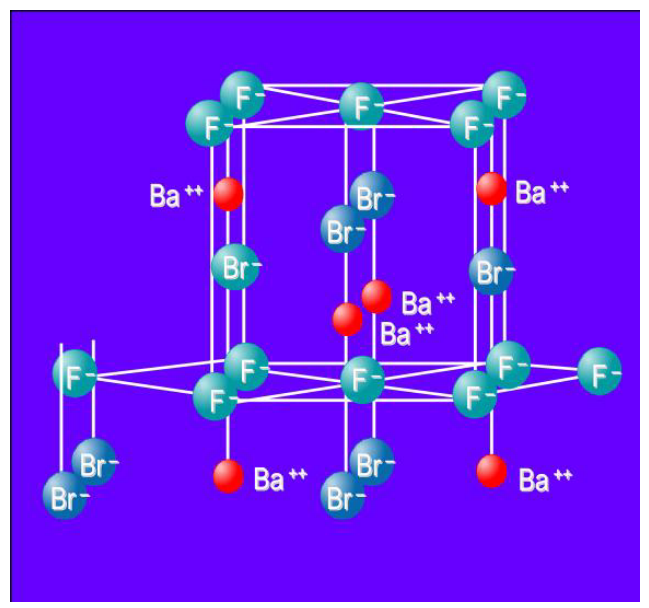

Figure 2.7: BaFBr crystal structure [20].

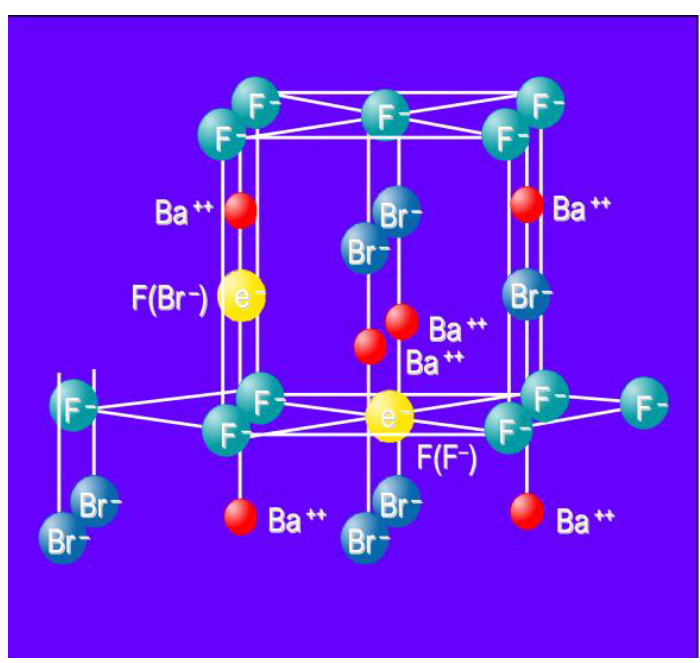

(a)

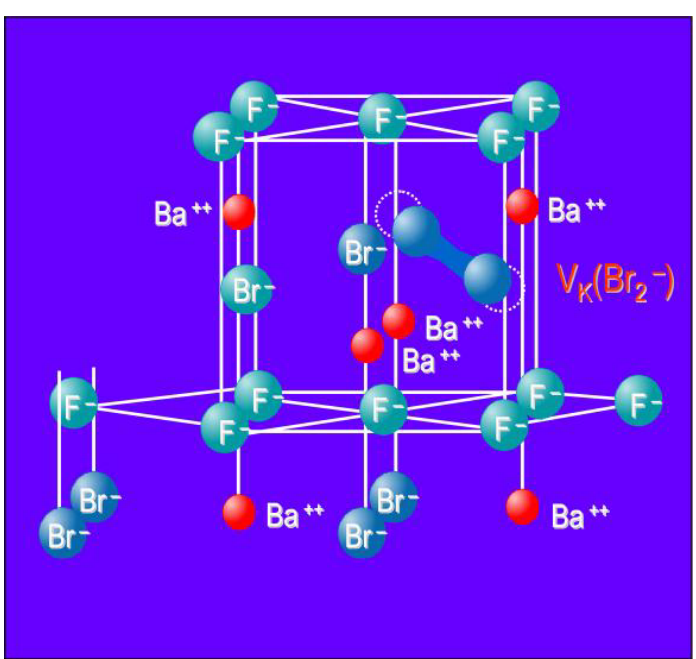

(b)

Figure 2.8: BaFBr crystal structure showing (a) $\mathrm{F}\left(\mathrm{Br}^{-}\right)$and $\mathrm{F}\left(\mathrm{F}^{-}\right)$centres and (b) $\mathrm{V}_{\mathrm{k}}$ centre [20].

structure where the $\mathrm{Ba}^{2+}$ ions are surrounded by a cage of four $\mathrm{F}^{-}$and four $\mathrm{Br}^{-}$

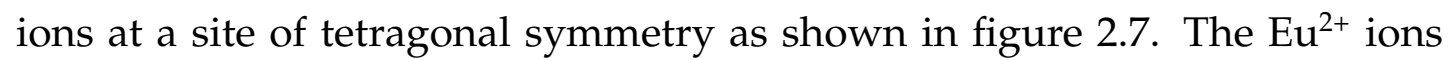
replace some of the $\mathrm{Ba}^{2+}$ ions within the host lattice.

For the PSL mechanism to occur both an electron and a hole trap need to be present. As explained in section 2.2.2 the electron trap is typically an anion vacancy, and since $\mathrm{BaFBr}$ has two negative ions, both $\mathrm{F}\left(\mathrm{Br}^{-}\right)$and $\mathrm{F}\left(\mathrm{F}^{-}\right)$centres (figure 2.8(a)) are possible. The hole centre is thought to be a $V_{k}$ centre (figure 2.8(b) , which has a hole trapped by two adjacent $\mathrm{Br}^{-}$lattice ions; both ions shift towards each other. For a more detailed discussion of the PSL mechanisms for $\mathrm{BaFBr}: \mathrm{Eu}^{2+}$ the reader is referred to Leblans et al. [20]. 


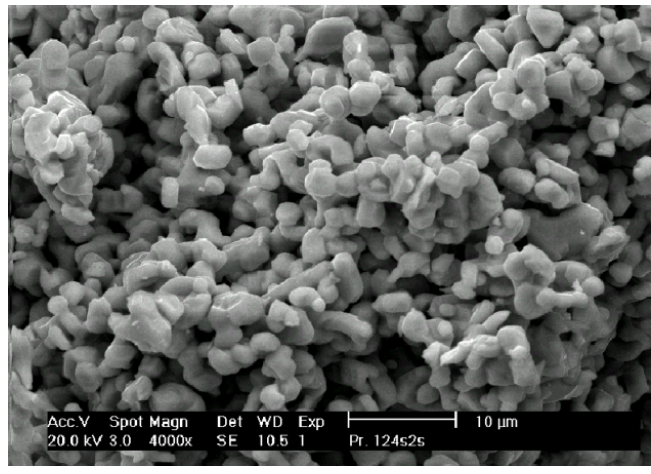

(a)

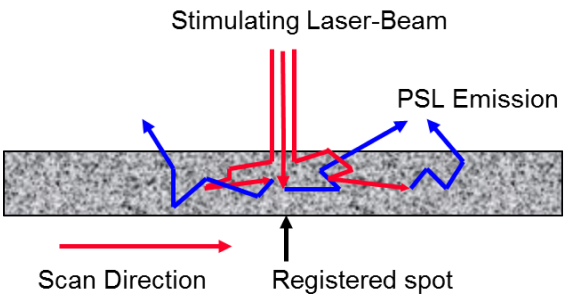

(b)

Figure 2.9: (a) SEM image of $\mathrm{BaFBr}: \mathrm{Eu}^{2+}$ imaging plate and (b) schematic of scattering within imaging plate [21].

Although BaFBr:Eu ${ }^{2+}$ has good storage phosphor performance, an alternative was sought to compete with new technologies, and to avoid the commercial limitation of the high number of Fuji patents on $\mathrm{BaFBr}_{\mathrm{Eu}}{ }^{2+}$ [20]. Direct radiography systems use a lower patient dose as compared to $C R$, which requires any new CR system to have a higher light output than $\mathrm{BaFBr}: \mathrm{Eu}^{2+}$ for the same absorbed dose to be competitive. This can be achieved by either using a phosphor with a higher X-ray absorption, or having a thicker phosphor layer. With powdered phosphor materials the thickness cannot be increased without significantly decreasing the resolution; the resolution is limited by scattering from the powder grains causing a larger than intended area to be stimulated. Figure 2.9 shows the grain structure of a BaFBr:Eu ${ }^{2+}$ imaging plate and the effects of scattering.

With regard to materials with a higher X-ray absorption coefficient, some alternative storage phosphor materials have been considered. These include $\mathrm{BaFBr}$ analogues such as $\mathrm{BaFCl}$, alkaline earth sulfides [20], and translucent glass ceramic materials [22]. All these showed promise but still do not have the performance of BaFBr:Eu ${ }^{2+}$. However, another possibility in the form of needle structured alkali halides (CsI, CsBr) was discovered which showed a higher resolution and could be made thicker to have a higher X-ray absorption [20]. The best performing needle imaging plate is based on caesium bromide doped 
with divalent europium $\left(\mathrm{CsBr}: \mathrm{Eu}^{2+}\right)$.

\subsection{Caesium bromide imaging plates}

The first storage phosphor based on caesium bromide was in single crystal form and doped with monovalent indium or gallium [23]. It was later found that doping with divalent europium [11, 19] gave a much better performance in terms of spatial resolution and light output for a given X-ray dose (conversion efficiency) and the stimulation intensity required to read-out the image (stimulation energy).

Commercial CsBr:Eu ${ }^{2+}$ imaging plates are made by thermal vapour deposition [20] (figure 2.10). The raw materials of $\mathrm{CsBr}$ salt and an activator compound (eg, $\mathrm{EuOBr})$ are heated above their melting temperatures in a vacuum chamber. A vapour cloud forms above the melted starting materials which then condenses on a cold substrate placed above the crucible. The imaging plate forms as a crystalline material in the form of needles perpendicular to the substrate, hence the name needle imaging plates. The size and formation of the needles depends on the pressure and the type of substrate used [24]. Figure 2.11 shows a topdown and side-on view of a $\mathrm{CsBr}: \mathrm{Eu}^{2+}$ imaging plate with the needle structure clearly visible. The improvement in resolution comes from the needles partially

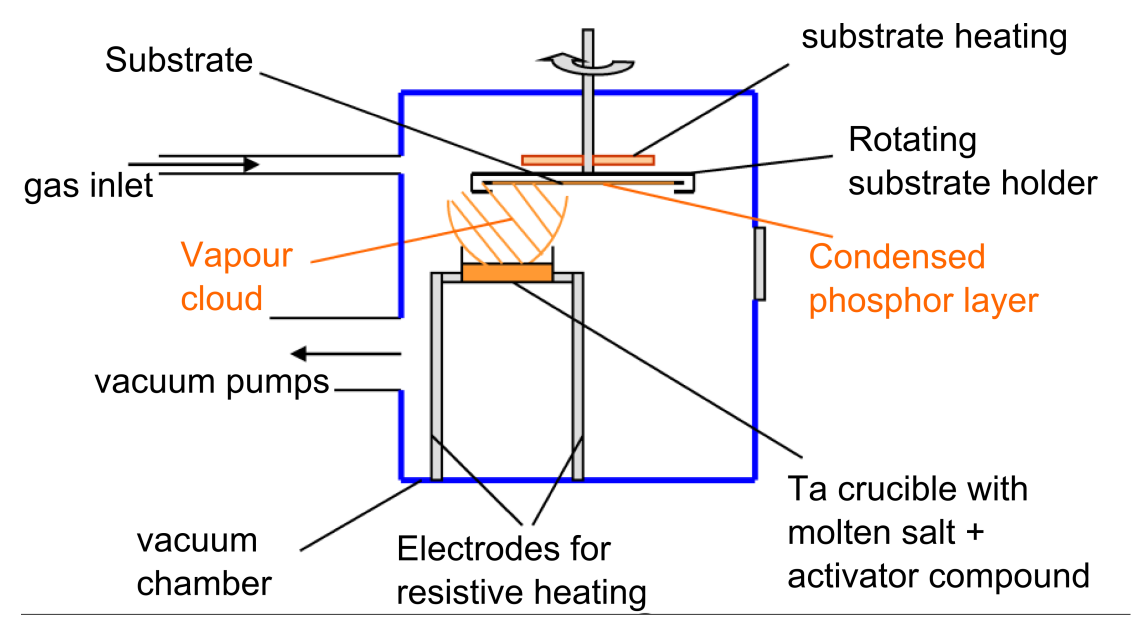

Figure 2.10: Schematic of thermal vapour deposition technique used for needle imaging plate manufacture [20]. 


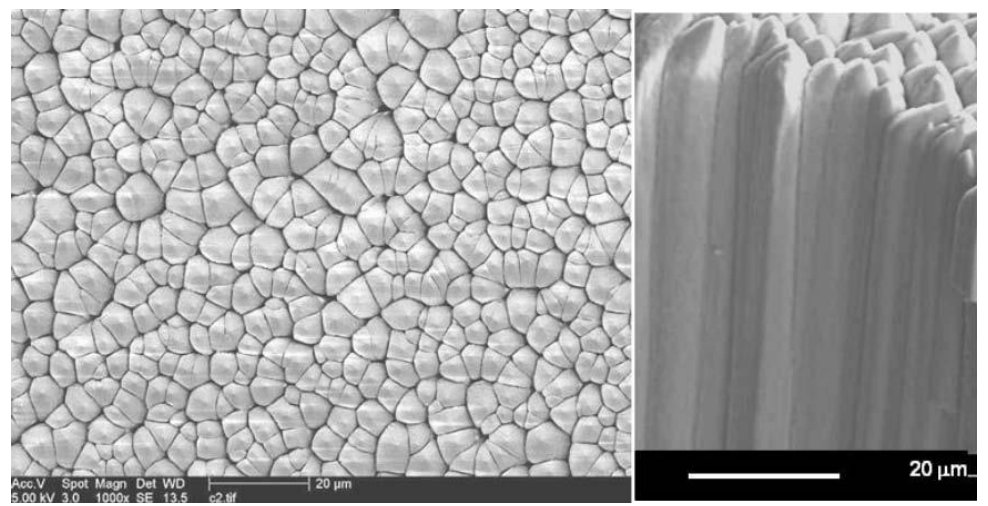

Figure 2.11: SEM images of $\mathrm{CsBr}_{\mathrm{Eu}}{ }^{2+}$ imaging plate [20], (left) top view and (right) side view.

guiding the stimulation light which reduces the scattering.

Stimulation and emission spectra for $\mathrm{CsBr}: \mathrm{Eu}^{2+}$ are shown in figure 2.12. The peak of the emission spectrum occurs at $440 \mathrm{~nm}$ and is due to the $4 f^{6} 5 d^{1} \rightarrow 4 f^{7}$ transition of the europium ion; this wavelength allows efficient detection with either a photomultiplier tube or a CCD detector. The stimulation spectrum peaks around $680 \mathrm{~nm}$, which is characteristic of electrons trapped in bromine vacancies, hence the electron traps are $\mathrm{F}\left(\mathrm{Br}^{-}\right)$centres [20]. The hole trapping mechanism is still widely debated, as discussed below.

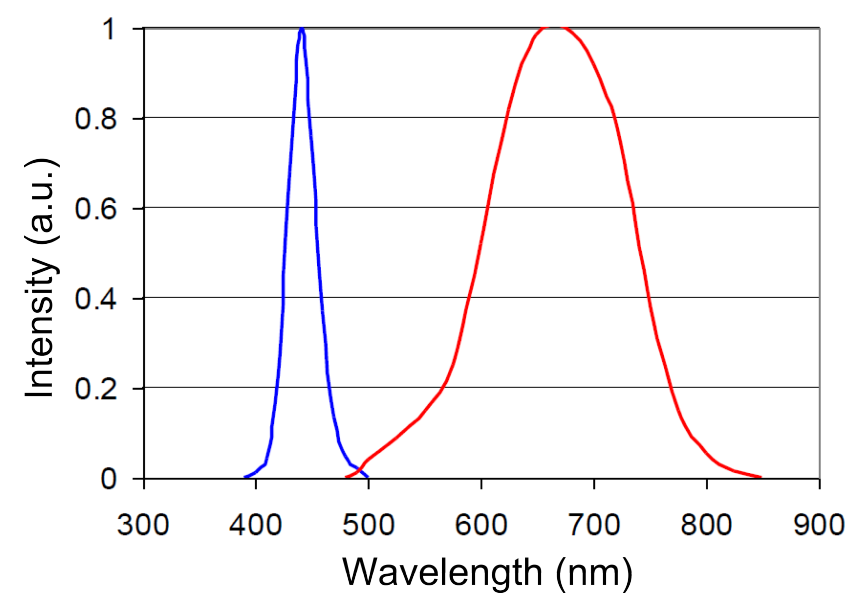

Figure 2.12: Normalised emission (blue curve) and stimulation (red curve) spectra for $\mathrm{CsBr}: \mathrm{Eu}^{2+}$ [20] 


\section{Hole traps}

Hackenschmied et al. [25] suggested that the hole trap must consist of an $\mathrm{Eu}^{2+}$ ion with a hole nearby. When an electron from a F centre is released it recombines with the hole, the energy of which is transferred to the $\mathrm{Eu}^{2+}$ ion and luminescence occurs. Annealing $\mathrm{CsBr}: \mathrm{Eu}^{2+}$ in air at temperatures up to $200{ }^{\circ} \mathrm{C}$ increases the PSL intensity [26], which was found to correlate with the formation of secondary phases. These phases were thought to be $\mathrm{CsEuBr}_{3}$ and $\mathrm{Cs}_{4} \mathrm{EuBr}_{6}$, and it was claimed that one of these phases causes better localisation of the charge carriers, hence the increase in PSL intensity. Annealing at temperatures above $250{ }^{\circ} \mathrm{C}$ ("over-annealing") causes an annihilation of the phase and a decrease in PSL intensity. Annealing in air leads to a higher PSL intensity compared to annealing in nitrogen, and it was suggested the hole trap in the secondary phase was similar to the $\left[\mathrm{Eu}^{2+}-\mathrm{O}^{-}\right]$centre formed in $\mathrm{BaFBr}: \mathrm{Eu}^{2+}[26]$. A later paper by Hesse et al. [27] found the secondary phases suggested by Hackenschmied et al. [25] exist prior to annealing and were not affected by the annealing process. Further, $\mathrm{CsEuBr}_{3}$ degrades rapidly to $\mathrm{Cs}_{2} \mathrm{EuBr}_{5} \cdot 10 \mathrm{H}_{2} \mathrm{O}$, which has europium in its trivalent state and therefore does not contribute to PSL. The explanation put forward by Hackenschmied et al. [26] therefore seems unlikely.

Hesse et al. [28] suggest that oxygen is the key to PSL, and that the oxygen forms a $\langle 111\rangle$ orientated $\left[\mathrm{Eu}^{2+}-\mathrm{O}^{2-}\right]$ electric dipole, where the $\mathrm{O}^{2-}$ ion sits on a bromine site in the vicinity of the $\mathrm{Eu}^{2+}$ ions. In this scenario the dipoles create strong electric fields which attract the hole towards the $\mathrm{Eu}^{2+}$ ion where it is trapped locally creating an $\mathrm{Eu}^{2+}$-hole complex. Annealing in a sulphur atmosphere was found to increase the PSL more than for air, which was attributed to the larger size of the sulphur ion creating a larger dipole with an increased attraction radius. However, no indication of the $\left[\mathrm{Eu}^{2+}-\mathrm{O}^{2-}\right]$ centre has been seen by electron paramagnetic resonance studies, so this explanation is also improbable. Appleby et al. [29, 30] investigated the effects of oxygen and water on the PSL. 
It was found that annealing the imaging plate (or powder) increases the PSL by up to a factor of 25. The hole traps are said to be $V_{k}$ centres in the vicinity of a $\left[\mathrm{Eu}^{2+}-\mathrm{O}^{2-}\right]$ dipole. Hydration was claimed to result in one molecule of $\mathrm{H}_{2} \mathrm{O}$ per unit cell which is oriented by the dipole field of the $V_{k}$ centre, increasing the attraction radius of the centres, and increasing the PSL. However, in this author's view, this model is unlikely as one water molecule per unit cell would cause the density of the material to differ markedly and measurably from pure $\mathrm{CsBr}$, and generate a distinctly different $\mathrm{XRD}$ pattern to anhydrous $\mathrm{CsBr}$, contrary to observation. PSL was also found in undoped $\mathrm{CsBr}$ samples and is attributed to the oxygen impurities incorporated during production. Further studies on oxygen impurities were also undertaken by the same authors [31, 32]. Loncke et al. [33, 34, 35] studied the electron paramagnetic resonance (EPR) of $\mathrm{CsBr}: \mathrm{Eu}^{2+}$ imaging plates. Previous studies found $\mathrm{CsBr}: \mathrm{Eu}^{2+}$ single crystals showed no EPR signal [19], but the needle imaging plates were found to have an EPR spectrum consistent with the needles having a $\langle 100\rangle$ crystal orientation. A tetragonal symmetry site (AA-EPR) was found which is not consistent with the hole traps being isolated $\mathrm{Eu}^{2+}$ ions or $\mathrm{Eu}^{2+}-\mathrm{V}_{\mathrm{k}}$ dipoles. Instead the hole trap consists of $\mathrm{a} \mathrm{Eu}^{2+}$ ion with a nearby lattice defect or impurity which stabilises against aggregation. The EPR spectra as a function of annealing temperature were found to correlate with the changes in intensity of the PSL [33]. Annealing at temperatures over $250{ }^{\circ} \mathrm{C}$ reduces the PSL intensity and a different EPR spectrum is seen. At low temperature, features of two different EPR spectra were found [35], a tetragonal symmetry site (AA-EPR I) and an orthorhombic symmetry site (AA-EPR II). The discovery of these sites rules out $\mathrm{Eu}^{2+}$ aggregates as the hole centre, as aggregates would not show a single-ion EPR spectrum. It is also not likely to be $\mathrm{Eu}^{2+}-\mathrm{V}_{\mathrm{k}}{ }^{-}$centres as they cannot explain the temperature dependence [36]. A model was first suggested where the hole centre consists of a interstitial $\mathrm{Eu}^{2+}$ ion at the centre of four $\mathrm{Br}^{-}$ions with two $\mathrm{H}_{2} \mathrm{O}$ ligands along the axis perpendicular to the plane (figure 2.13(a) and (b)). 
At high temperatures the $\mathrm{H}_{2} \mathrm{O}$ ligands rotate giving the (on average) tetragonal symmetry site (AA-EPR) and at low temperatures the rotation is frozen with the $\mathrm{H}_{2} \mathrm{O}$ ligands perpendicular to each other (AA-EPR I, figure 2.13(a)) or in the same plane as each other (AA-EPR II, figure 2.13(b)).

Electron nuclear double resonance (ENDOR) experiments were performed by Vrielinck et al. [36] to further determine the role of water as a hole trap. ENDOR spectra were found corresponding to ${ }^{1} \mathrm{H}$ nuclei which is most likely due to $\mathrm{H}_{2} \mathrm{O}$ molecules and not $\mathrm{OH}^{-}$ions. A second improved model of the europium centre and the role of water is shown in figure 2.13(c) and (d). In this model the europium ion substitutes for a caesium ion and has both a $\mathrm{H}_{2} \mathrm{O}$ molecule and a caesium vacancy in one of the next nearest neighbour positions. At low temperatures the orientation of the $\mathrm{H}_{2} \mathrm{O}$ with respect to the principal axis gives the AA-EPR I (figure 2.13(c)) and AA-EPR II (figure 2.13(d)) spectra. At high

(a)

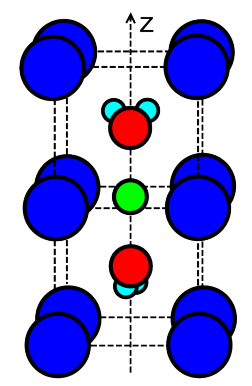

(c)

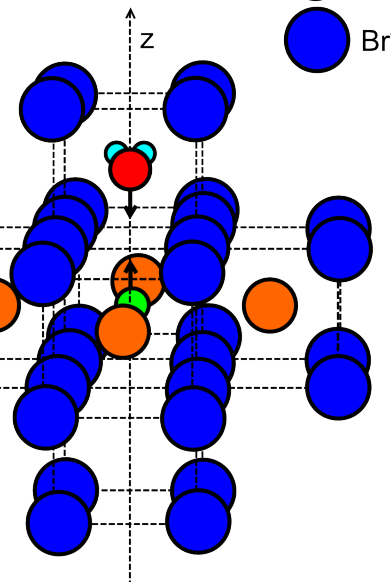

(b)

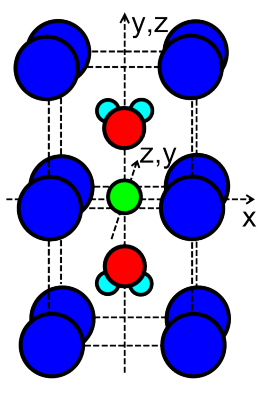

(d)

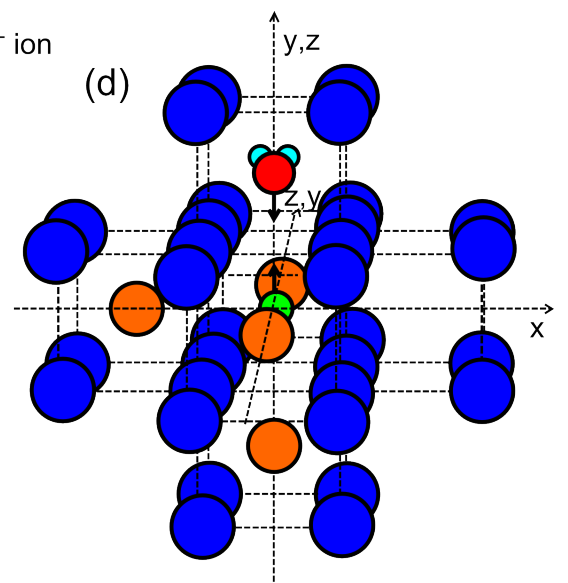

Figure 2.13: Models for the AA-EPR I [(a) and (c)] and AA-EPR II [(b) and (d)] centres [36]. Models (a) and (b) have the $\mathrm{Eu}^{2+}$ in an interstitial position and show (a) tetragonal symmetry and (b) orthorhombic symmetry. Models (c) and (d) have $\mathrm{Eu}^{2+}$ at a $\mathrm{Cs}^{+}$site and show (c) tetragonal and (d) approximately orthorhombic symmetry. 
temperatures the caesium vacancy can hop between cation positions around the europium, creating the tetragonal AA-EPR symmetry site. Currently this model is the most likely candidate for the hole centre in $\mathrm{CsBr}: \mathrm{Eu}^{2+}$. The EPR/ENDOR experiments provide definitive information on the site symmetry and the fact that it involves a molecule of water.

\section{Other dopants}

The first CsBr storage phosphors were single crystals doped with indium $\left(\mathrm{In}^{+}\right)$ or gallium $\left(\mathrm{Ga}^{+}\right)$[23]. These showed a PSL emission in the green at 504 and $515 \mathrm{~nm}$ for $\mathrm{In}^{+}$and $\mathrm{Ga}^{+}$respectively. Both of these dopants were found to be efficient storage phosphors but showed a much lower light output than for $\mathrm{BaFBr}: \mathrm{Eu}^{2+}$.

A later study by Rogulis et al. [37] also investigated the use of monovalent gallium as the dopant. Single crystals were grown by the Bridgman method with the gallium dopant added to the melt by using appropriate amounts of $\mathrm{GaBr}_{3}$ and elementary gallium. The PSL emission was again found to peak at $515 \mathrm{~nm}$ and the stimulation curve peaks at around $640 \mathrm{~nm}$. Annealing the crystals at a temperature of $520{ }^{\circ} \mathrm{C}$ causes an increase in the PSL signal. The electron traps were found to be $\mathrm{F}\left(\mathrm{Br}^{-}\right)$centres and the hole centres were thought to be $\mathrm{Ga}^{2+}$ centres.

Kurata et al. [38] doped CsBr with either indium, europium, samarium, terbium, gadolinium or neodymium in the trivalent state. The powders were mixed and pressed into discs which were then sintered at temperatures ranging from 250 to $600{ }^{\circ} \mathrm{C}$. All dopants showed an emission peak at approximately $440 \mathrm{~nm}$, as for $\mathrm{Eu}^{2+}$, except for indium which peaked around $500 \mathrm{~nm}$. No further analysis on the additional dopants was performed as the europium doped sample showed the most intense PSL.

Zorenko et al. [39, 40] studied luminescence properties of $\mathrm{CsBr}$ single crystals doped with copper in the form of copper metal, $\mathrm{CuO}$ or $\mathrm{CuBr}_{2}$. The $\mathrm{CsBr}$ 
processed with metallic copper has a triple peaked PSL spectrum with the main PSL peaks occurring at 456, 513 and $585 \mathrm{~nm}$. Crystals doped with $\mathrm{CuO}$ show a similarly peaked PSL spectrum but with a much higher intensity; the lower intensity for the metallic doped crystal is thought to be due to the $\mathrm{Cu}$ not completely dissolving in the crystal. $\mathrm{CuBr}_{2}$ doped crystals show a single peaked PSL curve with the maximum intensity occurring at $457 \mathrm{~nm}$. None of these alternative dopants have proven to be competitive to europium for $\mathrm{CsBr}$ X-ray storage phosphor performance. 


\section{Chapter 3}

\section{Experimental Techniques}

\subsection{Sample preparation}

Powdered starting materials of CsBr (Sigma Aldrich, 99.999\%) and a dopant, most commonly $\mathrm{EuBr}_{2}$ (Alfa Aesar, 99.99\%), were milled in a stainless steel rolling jar containing $5 \mathrm{~mm}$ diameter zirconia toughened alumina (ZTA) milling balls using a custom-made roller mill (figure 3.1(a)). The starting materials were either raw or pre-sintered before rolling; the sintering process will be described in chapter 5.6. The roller mill is capable of unloaded speeds of $100-700 \mathrm{rpm}$ which decreases with increasing weight placed on the rollers. The powders are milled for approximately 2 hours to generate a fine powder with the dopant well dispersed. After milling the powder is sieved to an average particle size of less than $25 \mu \mathrm{m}$ using a sieve stack (mesh sizes 106, 52 and $25 \mu \mathrm{m}$ ) and placed into a custom-made sieve shaker (figure 3.1(b)). The sieve shaker uses a Vortex-Genie 2 vibration module as the basis of the shaking motion.

If required, the milled powder is further processed by annealing or hydrating and then pressed into discs or vice versa. To produce the discs, $200 \mathrm{mg}$ of powder is placed into a stainless steel die and pressed at 8 tonnes of force with a Specac 15 tonne hydraulic press for 2 hours under a 0.1 torr vacuum. The resulting discs have a diameter of $13 \mathrm{~mm}$ with a thickness of approximately 300 $\mu \mathrm{m}$. 


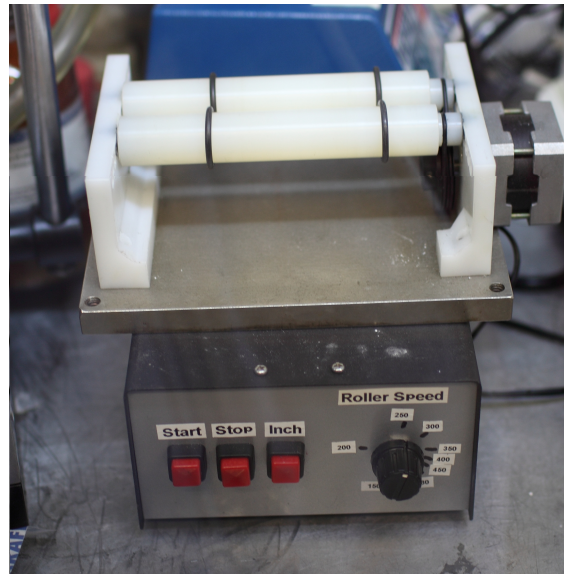

(a)

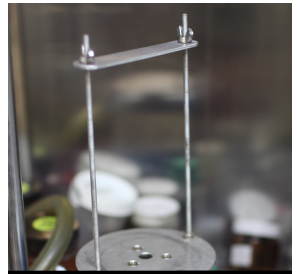

(b)

Figure 3.1: Equipment used to mill powders to less than $25 \mu \mathrm{m}$, (a) a custommade roller mill and (b) a sieve shaker based on a Vortex-Genie 2.

All sample preparation (apart from annealing and hydrating) was performed in a dry box. Initially, samples were prepared in a medium quality dry box, which has an argon atmosphere with approximately 25 ppm water. During the time of this work, a new MBRAUM UNIlab high quality dry box was acquired. This dry box has a nitrogen atmosphere with a water content of less than $0.1 \mathrm{ppm}$.

\subsection{X-ray sources}

A variety of X-ray sources were used due to the wide range and nature of the required applications.

An americium-241 radioactive source with an age-corrected activity of $3.4 \mathrm{GBq}$ $(\mathrm{Bq}=$ disintegrations per second) was used for PSL and DQE measurements. Americium-241 (Am-241) has a half life of 432.7 years and produces characteristic X-rays at energies of 13.9, 26.3 and $59.5 \mathrm{keV}$, with probabilities of 36.5, 2.7 and $35.9 \%$ respectively. The Am-241 is incorporated into a ceramic enamel (active ceramic) which is recessed into a tungsten alloy backing (to allow for backscatter applications) and sealed in a stainless steel capsule as shown in figure 3.2. The active ceramic has a diameter of $8 \mathrm{~mm}$, with the capsule having dimensions of $10.8 \mathrm{~mm}$ (diameter) and $5 \mathrm{~mm}$ (thickness). The stainless steel 


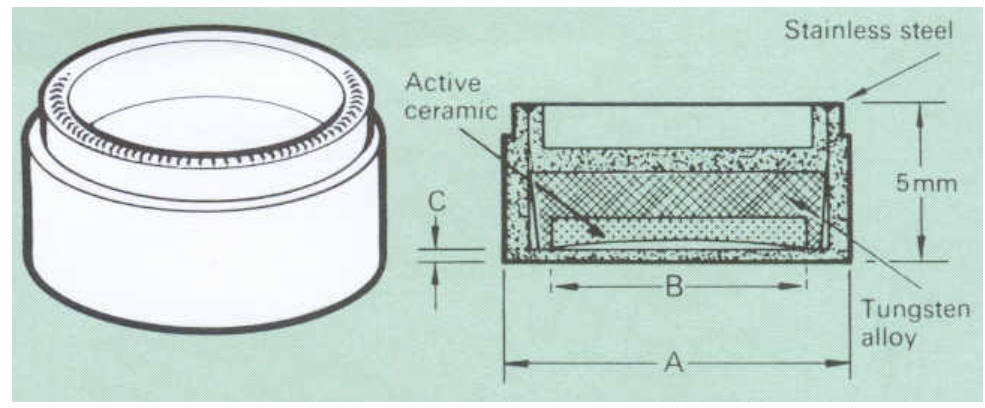

Figure 3.2: Americium-241 radioactive source schematic [41].

window covering the active ceramic has a thickness of $0.2-0.25 \mathrm{~mm}$.

A de Goetzen Xgenus dental X-ray set was used for testing of the camera imaging system (chapter 4) and for taking small X-ray images. The dental set has a tungsten tube with $2 \mathrm{~mm}$ aluminium filtration and is operated at $70 \mathrm{kV}$ and 8 $\mathrm{mA}$. Available exposure times range from $0.08-3.2 \mathrm{~s}$.

To capture large X-ray images, a Hewlett-Packard 43855A Faxitron was used. The Faxitron has a tungsten tube and is operated at a constant $0.3 \mathrm{~mA}$ current and adjustable $10-55 \mathrm{kV}$ output voltage. A very small focal spot (50 $\mu \mathrm{m})$ allows high resolution images to be produced.

Dual-energy imaging was performed using Golden Engineering XR150 portable X-ray generator. This generator has a tungsten tube and is operated at $150 \mathrm{kV}$. It is a pulsed source where each count consists of three very short duration pulses (approximately $50 \mathrm{~ns}$ ); a maximum of 99 counts can be performed in one irradiation.

\subsection{Integrating sphere measurements of photostim- ulated luminescence}

The performance of an X-ray storage phosphor in terms of its photostimulated luminescence output is specified by the quantities "stimulation energy" and "conversion efficiency" [19]. The PSL intensity ( $\left.I_{\mathrm{PSL}}\right)$ decays from the initial 
value $I_{0}$ as more trapped electrons are liberated as,

$$
I_{\mathrm{PSL}}=I_{0} \exp \left(-\frac{t}{\tau}\right)
$$

where the decay constant $\tau$ is inversely proportional to the stimulating light intensity $\left(I_{\text {stim }}\right)$,

$$
\tau=\frac{\alpha}{I_{\text {stim }}}
$$

$\alpha$ is the "stimulation energy" (SE), which is the energy per unit area required to reduce the PSL intensity to $1 / e$ ( $37 \%$ ) of its initial value and is defined as,

$$
\mathrm{SE}=I_{\mathrm{stim}} \tau
$$

The conversion efficiency (CE) is defined as the total PSL energy released during photon stimulation $\left(E_{\text {tot }}\right)$ per unit absorbed X-ray dose,

$$
\mathrm{CE}=\frac{E_{\text {tot }}}{\text { absorbed X-ray dose }} \text {. }
$$

Table 3.1 shows some typical values of the SE and CE for various storage phosphor materials.

To measure these quantities an integrating sphere measuring system and process was developed in this work. An integrating sphere is a device for measur-

Table 3.1: Typical stimulation energies and conversion efficiencies for various storage phosphor materials [11, 20, 23].

\begin{tabular}{ccccc}
\hline Storage phosphor & $\begin{array}{c}\text { Simulation } \\
\text { wavelength } \\
(\mathrm{nm})\end{array}$ & $\begin{array}{c}\text { Emission } \\
\text { wavelength } \\
(\mathrm{nm})\end{array}$ & $\mathrm{SE}$ & $\mathrm{CE}$ \\
$\left(\mu \mathrm{J} \mathrm{mm}^{-2}\right)$ & $\left(\mathrm{pJ} \mathrm{mR}^{-1} \mathrm{~mm}^{-2}\right)$ \\
\hline BaFBr:Eu & 633 & 390 & 16 & 20 \\
& 680 & 390 & 28 & 14 \\
$\mathrm{CsBr}: \mathrm{Eu}^{2+}$ & 690 & 440 & 4 & 18 \\
$\mathrm{CsBr}: \mathrm{In}^{+}$ & 680 & 504 & 23 & 3 \\
$\mathrm{CsBr}: \mathrm{Ga}^{+}$ & 680 & 515 & 4 & 5 \\
$\mathrm{RbBr}: \mathrm{In}^{+}$ & 680 & 490 & 25 & 2 \\
$\mathrm{RbBr}: \mathrm{Ga}^{+}$ & 680 & 550 & 4 & 6 \\
\hline
\end{tabular}




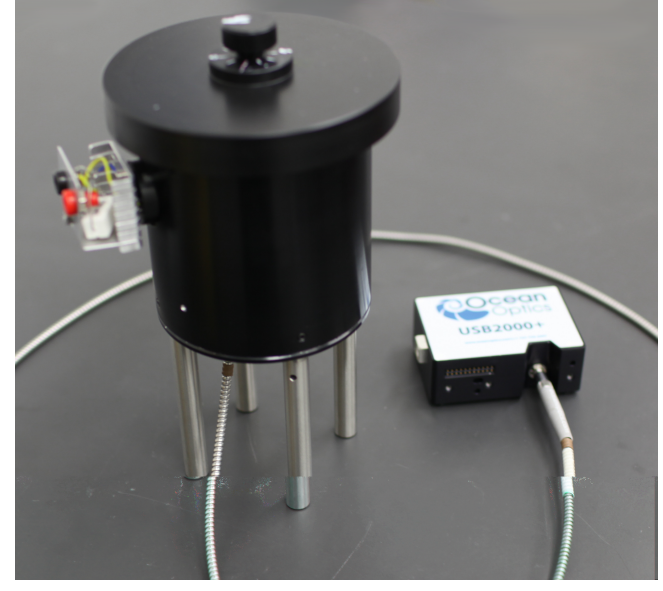

(a)

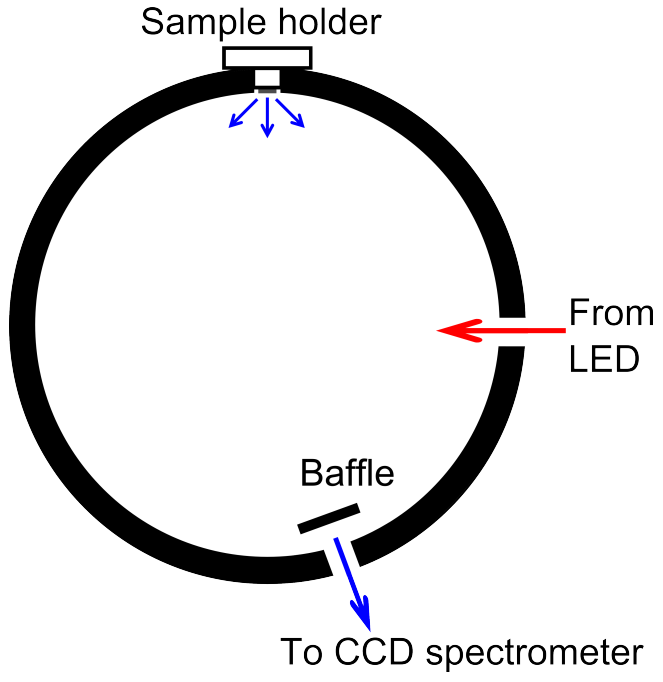

(b)

Figure 3.3: (a) Photo and (b) internal schematic of the integrating sphere measurement system.

ing optical radiation intensity which accommodates sources of light which do not produce single beams of radiation (such as a laser). It works by spatially integrating the radiant flux, that is, it diffuses the input light so that it spreads evenly over the surface area of the sphere. Every point within the sphere receives the same light intensity as every other point after sufficient scattering events. This allows for absolute light output measurements to be made.

The setup of the integrating sphere system is shown in figure 3.3 . The light from the stimulating LED is injected into the side of the integrating sphere. Light reaches the sample via multiple reflections from the sphere wall. The PSL emitted from the sample is also reflected off the walls and collected by a baffle-protected fibre optic cable. A baffle is used to ensure that light emitted directly from the sample is not collected, only that which has scattered from the walls. The fibre optic cable is fed into a CCD spectrometer, and the resulting spectrum is displayed on a computer.

An Ocean Optics Mikropack USB-ISP-80 integrating sphere was used which has a $80 \mathrm{~mm}$ internal diameter and a reflectivity of greater than $95 \%$ over the wavelength range of $200-2000 \mathrm{~nm}$, and $99 \%$ in the region of interest (350 - 600 $\mathrm{nm})$. 
The stimulating red LED is a Lumileds Luxeon Star with a typical wavelength of $636 \mathrm{~nm}$ and an output power of $1 \mathrm{~W}$. The LED is encased in a custom made holder (figure 3.4(a)) which has a thin tube to reduce the cross-section of the beam such that it is less than the area of the detector used (in an external measurement) to measure the LED light power. The rear of the casing has circuitry (figure 3.4(b) which controls the amount of current which flows through the LED. After controlling the current to $112 \mathrm{~mA}$ and restricting the beam via the small aperture size the power input into the sphere is $730 \mu \mathrm{W}$.

Samples are introduced into the integrating sphere using the sample holder shown in figure 3.4(c). The sample holder can be used for samples up to $8 \mathrm{~mm}$ in diameter and samples are mounted using optical grease. The sample holder has a screw fitting with a pitch of $1 \mathrm{~mm}$, where every $360^{\circ}$ turn corresponds to the sample moving $1 \mathrm{~mm}$ in the vertical direction. The sample holder is inserted into a top plate and the position is adjusted so that the samples top surface is aligned with the surface of the sphere.

A baffle-protected Ocean Optics silica fibre optic cable (see figure 3.4(d) was used to transfer the light from the integrating sphere to the CCD spectrometer. The CCD spectrometer is an Ocean Optics USB2000+ miniature fibre optic spectrometer (figure 3.4(e)). This spectrometer has a low cost, high performance 2048 element linear CCD, a spectral range of $190-890 \mathrm{~nm}$ and a resolution of 0.3 $\mathrm{nm}$. The spectrometer is connected to the computer via a USB cable and spectra are displayed using the SpectraSuite software provided by the manufacturer.

\subsubsection{Measurement process}

The measurement process can be split into three parts - irradiation of the sample, collection of the PSL spectrum and analysis of the data.

The sample is irradiated using an americium-241 radioactive source (see section 3.2) with a source to sample distance $(r)$ of $5 \mathrm{~mm}$. The $59.5 \mathrm{keV}$ photon is dominant at the sample position due to absorption of the lower energy X-rays 


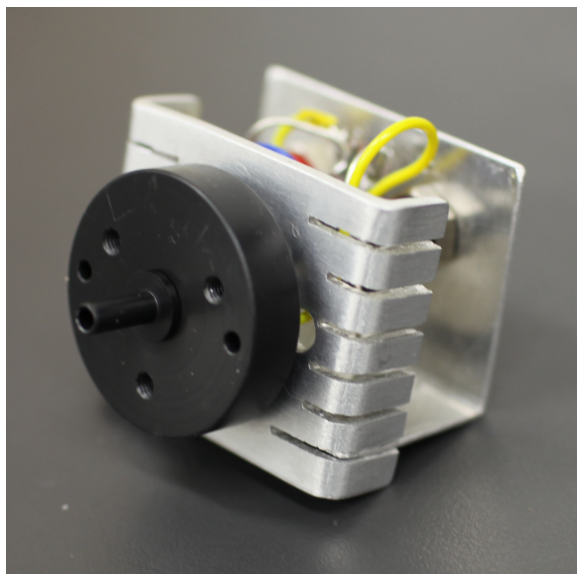

(a)

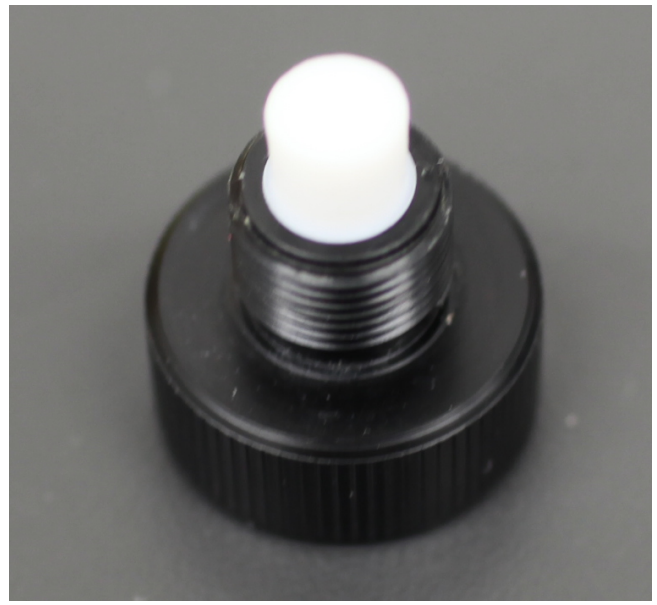

(c)

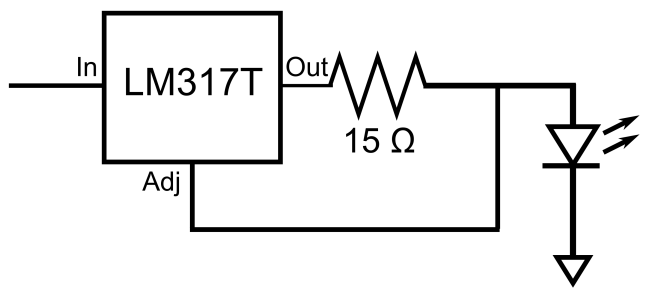

(b)

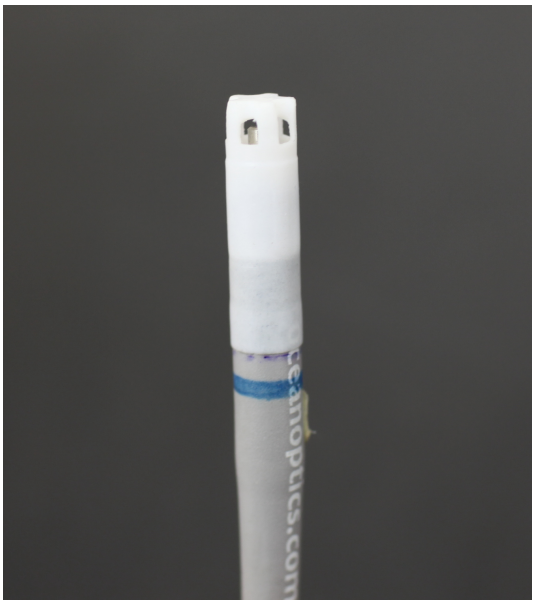

(d)

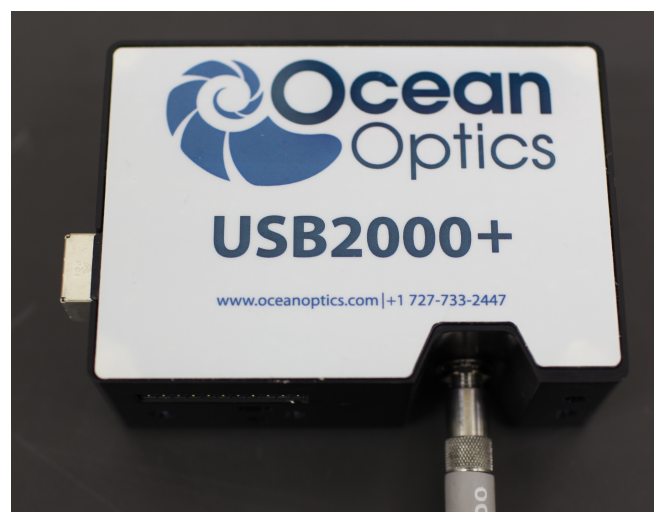

(e)

Figure 3.4: Integrating sphere components, (a) LED with casing, (b) current control circuit for LED, (c) sample holder, (d) fibre optic cable with baffle and (e) Ocean Optics USB2000+ spectrometer. 
in the outer casing of the source. The number of $59.5 \mathrm{keV}$ photons reaching the sample (with area $A$ ) per second is,

$$
\frac{d N}{d t}=R \frac{A}{4 \pi r^{2}} P_{\mathrm{E}} \exp (-\mu r)
$$

where $R$ is the source activity, $P_{\mathrm{E}}$ is the probability of the emitted photon having an energy of $59.5 \mathrm{keV}, \exp (-\mu r)$ is the attenuation of the photons due to material (air) between source and sample and $\mu$ is the material attenuation coefficient. In general the last term in equation 3.5 is negligible as the attenuation in air is miniscule over short distances; $99.99 \%$ of $59.5 \mathrm{keV} \mathrm{X-ray} \mathrm{photons} \mathrm{pass} \mathrm{through}$ $5 \mathrm{~mm}$ of air.

If the initial intensity of the X-ray beam (in $\mathrm{W} \mathrm{m}^{-2}$ ) falling on the surface of the sample is $I_{0}$, then the energy deposited in a tissue equivalent sample with area $A$ and thickness $d z$, in time $t$ is,

$$
\begin{aligned}
\left(I_{0}-I\right) A t & =\left[I_{0}-I_{0} \exp \left(-\left(\frac{\mu}{\rho}\right)_{\mathrm{tis}} \rho d z\right)\right] A t, \\
& \approx I_{0}\left(\frac{\mu}{\rho}\right)_{\mathrm{tis}} \rho d z A t
\end{aligned}
$$

where $(\mu / \rho)_{\text {tis }}$ is the tissue mass attenuation coefficient and $\rho$ the density of tissue. The initial intensity, $I_{0}$, depends on the energy $E$ of the X-ray photon (in Joules) and the number of photons reaching the sample (equation 3.5), and is given by,

$$
I_{0}=E \frac{d N}{d t}
$$

Therefore the energy deposited in a tissue equivalent sample per unit time is,

$$
\frac{\text { Deposited Energy }}{\text { time }}=E \frac{d N}{d t} M\left(\frac{\mu}{\rho}\right)_{\text {tis }},
$$

where $M$ is the mass of tissue ( $M=\rho A d z)$. The absorbed X-ray dose rate is the energy deposited per unit time per unit mass and is found by combining and 
simplifying equations 3.5 and 3.8 to give,

$$
\text { Dose rate }=\frac{R}{4 \pi r^{2}} E P_{\mathrm{E}} \exp (-\mu r)\left(\frac{\mu}{\rho}\right)_{\text {tis }},
$$

where the dose rate is in Sv/hr.

A comparison of the calculated dose rate to that measured using a calibrated Thermo Scientific radiation survey meter gives dose rates approximately 1.8 times larger $(0.52 \mathrm{~Sv} / \mathrm{hr}$ calculated compared to $0.29 \mathrm{~Sv} / \mathrm{hr}$ measured at a distance of $5 \mathrm{~mm}$ ). One reason for this discrepancy is absorption of photons by the stainless steel casing of the source. The thickness of stainless steel is between 0.2 and $0.25 \mathrm{~mm}$ but the actual composition of the steel and hence the attenuation coefficient is not known. Assuming a typical (304) stainless steel composition of around 70\% iron, 20\% chromium and 10\% nickel the rate calculated is $0.41 \mathrm{~Sv} / \mathrm{hr}$, still approximately 1.4 times higher than the measured rate. The remaining discrepancy is most likely caused by the uncertainty associated with the activity of the source only being quoted to one significant figure (100 $\mathrm{mCi})$. Therefore, the survey meter was chosen as the more reliable and accurate measurement of the actual dose received (the meter measures doses from 10 $\mathrm{nSv} / \mathrm{h}$ to $1 \mathrm{~Sv} / \mathrm{h}$ with a claimed $5 \%$ accuracy).

For an adequate signal-to-noise ratio and exposure times which are significantly less than the X-ray storage phosphor storage times, the sample has to be approximately $5 \mathrm{~mm}$ away from the source. The design of the radiation survey meter means there is a minimum distance of approximately $25 \mathrm{~mm}$ between the source being measured and the detector. The dose rate was measured as a function of distance, the results of which are shown in figure 3.5. Due to the finite width of the source $(8 \mathrm{~mm}$ in diameter) being comparable with the source to sample distance, a simple inverse square relationship cannot be used. Therefore, the equation for the dose rate is calculated for a disc source of radius $a=4 \mathrm{~mm}$, placed a distance $r$ along the axis from a small sample. A simple 


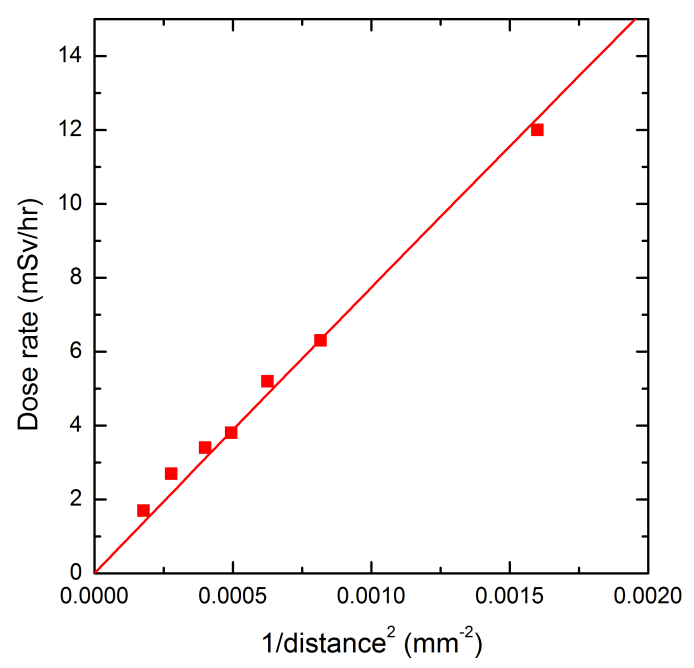

Figure 3.5: Dose rate as a function of distance from source to sample.

integral based on the inverse square law gives,

$$
\text { Dose rate }=K \ln \left(1+\frac{a^{2}}{r^{2}}\right)
$$

The coefficient $K$ is determined by least squares fitting the dose rate versus distance data with equation 3.10 (shown in figure 3.5). This gives a value of $K=487 \mathrm{~Sv} / \mathrm{hr}$, and equation 3.10 has been used with this value of $K$ to determine the dose rates in the remainder of this thesis.

After irradiation the sample is transferred to the integrating sphere in the dark. The LED is turned on and the wavelength-integrated PSL decay signal

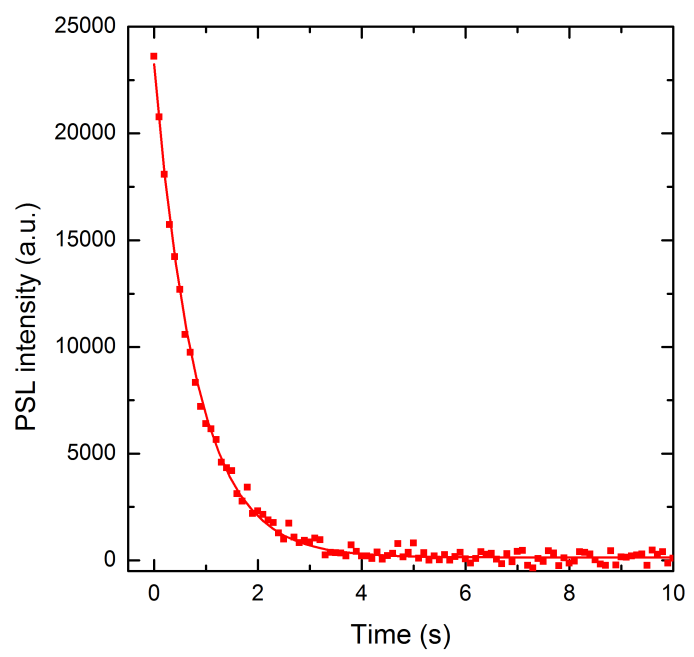

Figure 3.6: Example of a PSL decay curve. 
is recorded as a function of time by the computer. An example of a PSL decay curve is shown in figure 3.6. An integration time of $100 \mathrm{~ms}$ was used for the measurement.

\subsubsection{Integrating spheres}

In order to analyse the data, the basics of integrating sphere theory need to be discussed. As mentioned earlier an integrating sphere diffuses the input light so it is evenly spread over the entire surface area of the sphere. This is achieved through both the sphere shape and a highly reflective Lambertian reflectance surface. The input light on the reflective surface creates a virtual light source on reflection and the light from this can be described by its luminance. The luminance $L$ is a measure of the brightness of a Lambertian surface, and is defined by the radiant flux (optical power) $d \phi$ from a surface element $d A$, emitted within a solid angle $d \Omega$ at an angle $\theta$ to the normal to the surface (shown in figure $3.7(\mathrm{a})$. The luminance is given by,

$$
L=\frac{d \phi}{d A d \Omega \cos \theta}
$$

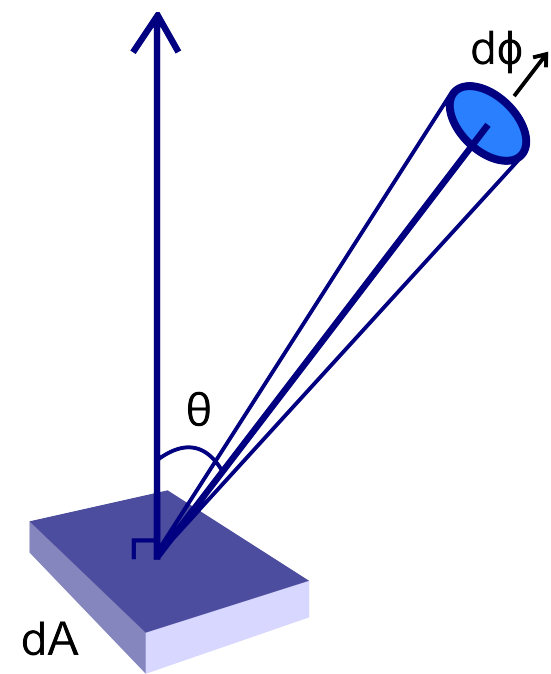

(a)

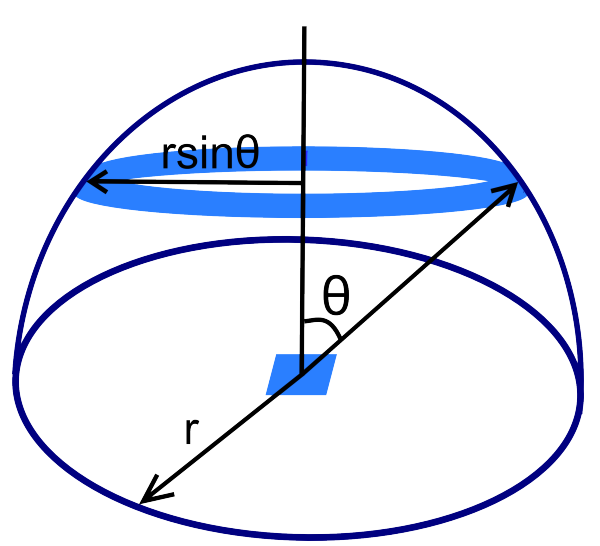

(b)

Figure 3.7: Visual representations of (a) $\mathrm{d} \phi$, power into solid angle $\mathrm{d} \Omega$, and (b) integral over surface area. 
The total flux emitted over a hemisphere can be found by integrating (figure 3.7(b) to give,

$$
\phi=\pi L d A
$$

In an integrating sphere, radiant power $\phi_{\text {in }}$ enters the sphere (in the form of light) and in a simple view is distributed uniformly over the entire surface area. If the flux was absorbed somehow, the flux per unit area would be $\phi_{\text {in }} / A_{s}$, where $A_{s}$ is the surface area of the sphere. Instead, if the flux was reflected the luminance would be,

$$
\begin{aligned}
\pi L d A & =\frac{\phi_{\text {in }}}{A_{\mathrm{s}}} d A, \\
\text { or } \quad L & =\frac{\phi_{\text {in }}}{\pi A_{\mathrm{s}}} .
\end{aligned}
$$

However, the multiple reflections off the sphere walls means that the luminance is greater than that in equation 3.13 by a multiple $M$ (multiplication factor), which is typically between 10 and 100. For an integrating sphere with a fraction $f$ of the surface removed for surface ports (port fraction) and a surface reflectivity $\rho$, the luminance is calculated to be [42],

$$
L=\frac{\phi_{\text {in }}}{\pi A_{\mathrm{s}}} M
$$

where,

$$
M=\frac{\rho}{1-\rho *(1-f)}
$$

The integrating sphere used in this work has a port fraction of 0.003 , and the manufacturer states the walls are made of sintered PTFE, but does not specify $M$ or the actual surface reflectivity at a given wavelength. From [43] the reflectivity was determined to be 0.99 over the wavelength range of interest, and the sphere multiplier was calculated to be $76 \pm 5$.

The sphere multiplier can also be measured from the flux escaping from one of the ports, by measuring the input and output powers using a calibrated optical 


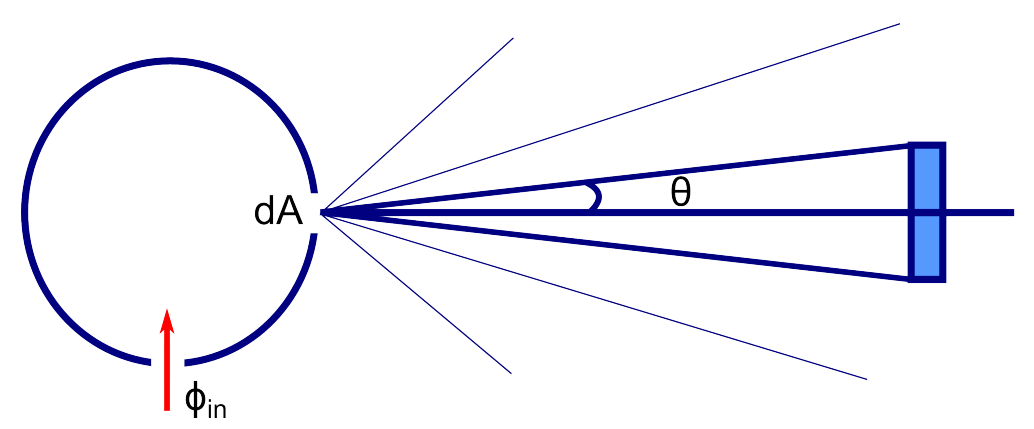

Figure 3.8: Visual representation of power emitted from area $d A$ falling on a detector.

power meter (Thorlabs PM100). The power received by the detector, subtended by angle $\theta$ by the radius of the detector circle, from a port opening with area $d A$ (shown in figure 3.8 ) is given by,

$$
\begin{aligned}
\phi_{\text {out }} & =\int_{\text {cone }} L \cos \theta d A d \Omega, \\
& =\pi L d A \sin ^{2} \theta, \\
& =\frac{\phi_{\text {in }} M}{A_{\mathrm{s}}} d A \sin ^{2} \theta .
\end{aligned}
$$

The magnification is then found by rearranging equation 3.16 to give,

$$
M=\frac{\phi_{\text {out }}}{\phi_{\text {in }}} \frac{A_{\mathrm{s}}}{d A \sin ^{2} \theta} .
$$

This yields a magnification factor of 80 , in excellent agreement with the value estimated from the port fraction and the reflectivity, and this is the value used for the remainder of this thesis.

\subsubsection{Analysis}

\section{Stimulation energy}

To compute the stimulation energy the intensity of the stimulating LED light falling on the sample is required. If the area occupied by the sample (area $d A$ ) was part of the integrating sphere surface, power balance would lead to the 
incident flux being the same as the scattered flux. That is,

$$
\begin{aligned}
\phi_{\text {incident }} & =\pi L d A, \\
& =\frac{\phi_{\text {in }}}{A_{s}} M d A .
\end{aligned}
$$

If the section of sphere is removed and replaced with the same area sample, and assuming negligible perturbations to the sphere, the incident intensity is,

$$
\begin{aligned}
I & =\frac{\phi_{\text {incident }}}{d A}, \\
& =\frac{\phi_{\text {in } M}}{A_{\mathrm{s}}} .
\end{aligned}
$$

The decay constant $\tau$ is found by fitting the PSL decay curve (for example figure 3.6) with a single exponential, as given in equation 3.2 . Therefore, from equations 3.3 and 3.19 , the stimulation energy is,

$$
\mathrm{SE}=\frac{\phi_{\mathrm{in}} M \tau}{A_{\mathrm{s}}}
$$

\section{Conversion efficiency}

The calculation of the conversion efficiency (equation 3.4 requires knowledge of the total PSL energy released for a given absorbed X-ray dose. The absorbed $\mathrm{X}$-ray dose in Sv is found using equation 3.10 and is converted into the Roentgen (R) unit by multiplying by $113.6 \mathrm{R} / \mathrm{Sv}$ [44].

In simple terms, the total PSL energy released is the area under the decay curve (figure 3.6). This can be fitted with a decay curve of the form,

$$
I=I_{0} \exp \left(-\frac{t}{\tau}\right)
$$

which has the analytical integral,

$$
\int_{0}^{\infty} I_{0} \exp \left(-\frac{t}{\tau}\right) d t=\tau I_{0}
$$




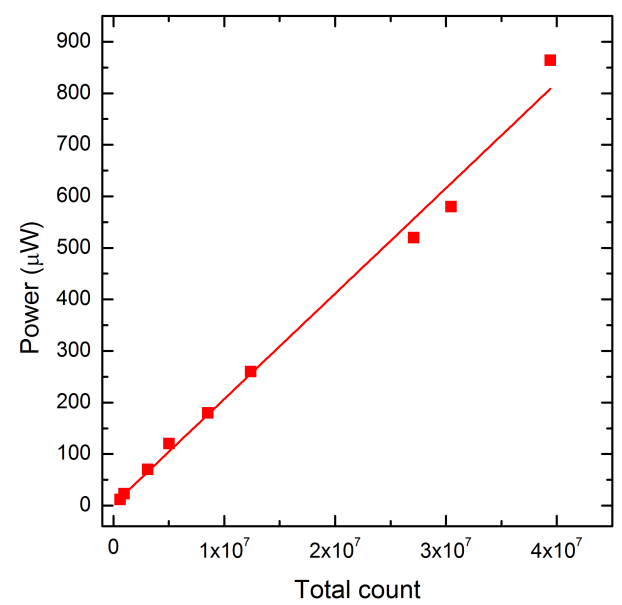

Figure 3.9: Power versus total count for a 405 nm LED.

The total PSL energy emitted $\left(E_{\text {tot }}\right)$ is then,

$$
E_{\text {tot }}=\tau I_{0}
$$

which has units of counts multiplied by time.

To calibrate the CCD spectrometer for intensity versus counts, a $405 \mathrm{~nm}$ LED was used whose power as a function of diode current was measured accurately with a calibrated laser power meter (Thorlabs PM100). A 405 nm LED was chosen as it has an emission which is in the same spectral region of the PSL emission for both BaFBr:Eu ${ }^{2+}(390 \mathrm{~nm})$ and $\mathrm{CsBr}: \mathrm{Eu}^{2+}(440 \mathrm{~nm})$. The LED emission band was integrated over wavelength by the CCD spectrometer, and the calibration curve in figure 3.9 was generated. The total PSL energy emitted, now with units of Joules is found using,

$$
E_{\text {tot }}(J)=2.14 \times 10^{-5} \times E_{\text {tot }}(\text { counts } \times \text { time }) .
$$

The pre-factor is the calibration constant which relates the energy (power $\mathrm{x}$ time) of the LED to the energy (integrated power) of the PSL sample. 


\section{Comparison with literature}

Results for a BaFBr:Eu ${ }^{2+}$ commercial imaging plate and a $\mathrm{CsBr}_{\mathrm{Bu}}{ }^{2+}$ test sample compared to literature [23, 11] are shown in table 3.2. Note that these values for the $\mathrm{CE}$ also take into account the thickness of the sample and so are given in units of $\mathrm{pJmR}^{-1} \mathrm{~mm}^{-3}$. For BaFBr:Eu ${ }^{2+}$ the materials are both commercial imaging plates (powder in resin), whereas for $\mathrm{CsBr}: \mathrm{Eu}^{2+}$ a hydrated pressed pellet was compared with a needle imaging plate, as no small piece of needle imaging plate was available. The conversion efficiency for the needle imaging plate was measured by comparing the light output for the same dose of the commercial plate and the hydrated pressed pellet using the camera read-out system (chapter 4).

The conversion efficiencies for the integrating system are markedly lower than those reported in literature, which is attributed to the different measurement techniques. The measurement techniques in the literature are not discussed at all, but are likely to be performed with focussed laser scanning stimulation, fibre-optic pick up and poly-energetic X-ray irradiation with unknown filtration. The differences in the CE and SE values presented here, and those in literature can arise from the following.

- The absorption of X-rays by the samples is dependent on the energy of the X-rays. This is well defined for a mono-energetic source, such as the 59.5 $\mathrm{keV} \mathrm{X}$-rays from the americium source, but for a poly-energetic source

Table 3.2: Comparison of literature CE and SE values [20] to those determined by the integrating sphere method. The imaging plates were a Fuji ST-VI BaFBr:Eu ${ }^{2+}$ imaging plate, a Konica Minolta CP1M200 needle imaging plate and a hydrated pressed pellet (made in this work).

\begin{tabular}{ccccc}
\hline Storage phosphor & $\begin{array}{c}\mathrm{SE} \\
\mu \mathrm{J} \mathrm{mm}^{-2}\end{array}$ & $\begin{array}{c}\mathrm{SE} \text { (lit) } \\
\mu \mathrm{J} \mathrm{mm}^{-2}\end{array}$ & $\begin{array}{c}\mathrm{CE} \\
\mathrm{pJ} \mathrm{mR}^{-1} \mathrm{~mm}^{-3}\end{array}$ & $\begin{array}{c}\mathrm{CE} \text { (lit) } \\
\mathrm{pJ} \mathrm{mR}^{-1} \mathrm{~mm}^{-3}\end{array}$ \\
\hline BaFBr:Eu$^{2+}$ & 6.9 & 16 & 4.3 & 29 \\
$\mathrm{CsBr}_{\mathrm{Eu}}^{2+}$ (pellet) & 2.1 & 4 & 6.7 & 37 \\
$\mathrm{CsBr}_{\mathrm{Eu}}^{2+}$ (needle plate) & - & 4 & 5.1 & 37
\end{tabular}


most of the low energy $X$-rays are absorbed while a significant fraction of the high energy $X$-rays are transmitted. This will affect the CE estimate in terms of the measured dose.

- For the integrating sphere the incident stimulation light is Lambertian in nature, whereas for laser scanning the light is incident normal to the surface. This will affect the stimulation energy through the power penetrating the sample.

- The collection optics for a fibre-optic pick up (presumably used in measurements for the literature values) will only collect a fraction of the PSL light emitted from the sample. This may or may not have been corrected for. With the method discussed here, all the emitted PSL light enters the integrating sphere, and any losses are corrected for by a calibration step. This will affect the conversion efficiency in terms of the total energy emitted from the sample.

The process which has been outlined and followed in this thesis yields CE and SE values though a protocol which is well-defined with no obvious flaws, and so the values can be quoted with some confidence. The differences in CE and SE values reported here and in the literature are attributed to different methodologies; those used for the literature values are unspecified. However, in practical terms, it is the relative values of the materials to be presented in chapter 5 compared to the "standard" $\mathrm{BaFBr}: \mathrm{Eu}^{2+}$ and $\mathrm{CsBr}: \mathrm{Eu}^{2+}$ imaging plates which is of importance. Therefore, any consistent and systematical method can be used to determine the relative values. For absolute values under well-defined conditions the values measured here are preferred.

\subsection{Determining imaging plate resolution}

The spatial resolution of an X-ray storage phosphor imaging plate or material is specified in terms of the modulation transfer function (MTF), which can be 
(a) lead grid

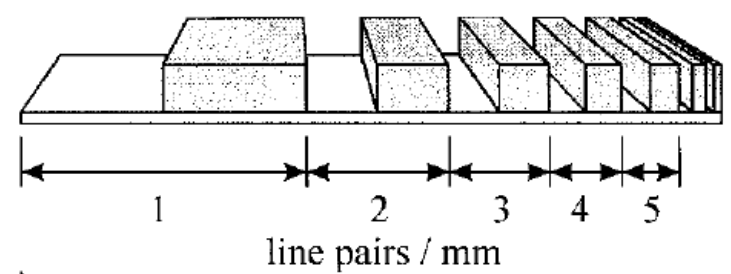

(b) ideal image

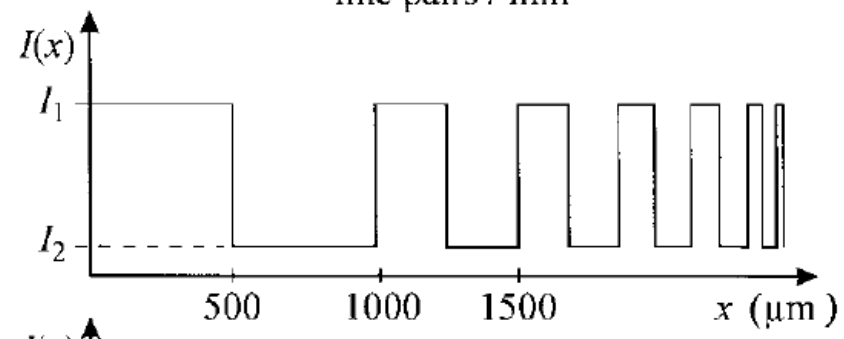

(c) real image

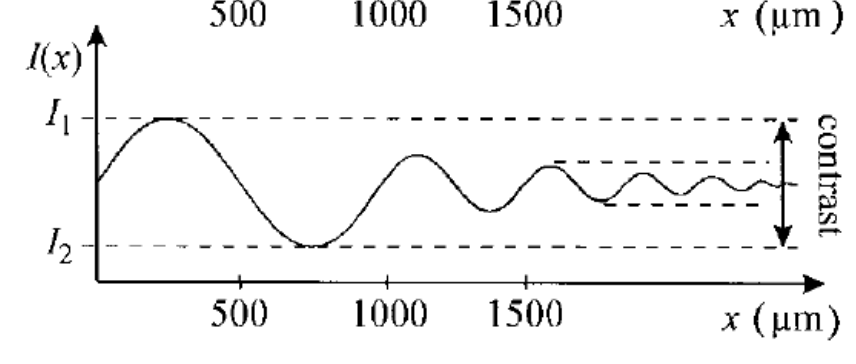

Figure 3.10: Determination of the contrast modulation and contrast transfer function by imaging a test grid [19].

measured by recovering and analysing the image of a test grid pattern. The test grid consists of a stepwise varying pattern of spatial frequencies, an example of which is shown in figure 3.10(a). For an ideal imaging system the contrast between the low and high intensities $\left(I_{1}\right.$ and $I_{2}$ respectively in figure $\left.3.10(b)\right)$ does not change with increasing spatial frequency. However, in a real image the contrast does depend on spatial frequency (figure 3.10(c)), the analysis of which gives the contrast transfer function (CTF) [19],

$$
\operatorname{CTF}(k)=\frac{C_{\text {real }}(l)}{C_{\text {ideal }}(k)}=\frac{C_{\text {real }}(k)}{\text { constant }}
$$

To convert the CTF into the MTF, each set of data for a given spatial frequency is least squares fitted with a sinusoidal function (see appendix B); the MTF assumes a sinusoidal variation in attenuation, not a square wave as in the CTF. 
The sinusoidal function is of the form,

$$
y=A \sin (2 \pi f x),
$$

where $A$ is the amplitude of the sinusoid, $f$ the spatial frequency of the line pairs. The MTF is then the amplitude $A$ for each spatial frequency, normalised to a value of 1 at a spatial frequency of zero. In many commercial applications the resolution is quoted as being the spatial frequency at which the MTF has fallen to a value of 0.2 .

Images of test grids were taken using the digital camera read-out system described in chapter 4 Analysis of the grids was then performed using MATLAB to generate the line of data, and Origin to perform the least squares fitting.

\subsection{Optical absorption measurements}

The interaction of electromagnetic (EM) radiation with matter can give useful information on the electronic and vibrational properties of that material. Radiation with wavelengths in the ultra-violet and visible range $(200-800 \mathrm{~nm})$ can induce transitions between different energy levels, such as between the ground and excited states of an ion, or between valence and conduction bands of a solid. These transitions are characteristic of the particular ion as perturbed from the free ion state by the crystal environment, or of the band structure. For longer wavelengths in the mid infra-red $(2.5-25 \mu \mathrm{m})$, radiation induces vibrations of molecules which can be used to extract the fundamental vibrational frequencies, or rotations of molecular groups in a sample.

Figure 3.11 shows schematically the passage of EM radiation through a material of thickness $z$. Normal incidence of the radiation and a slab of material with plane parallel faces is assumed, as this is the case in solid state optical spectroscopy. The initial intensity $I_{0}$ of the radiation decreases as it goes through the material. The first attenuation factor is reflection of the radiation from the front 
face of the material. The reflection coefficient is given by Fresnel's equation for normal incidence,

$$
R=\left(\frac{n_{1}-n_{2}}{n_{1}+n_{2}}\right)^{2}
$$

where $n_{1}=1$ and $n_{2}$ are the refractive indices for air and material respectively. For $\operatorname{CsBr} n_{2} \approx 1.7$ [45] and $6.7 \%$ of the initial intensity is reflected. Reflection also occurs at the back surface and so the total intensity reflected is $2 R(13.4 \%$ for $\mathrm{CsBr})$.

Within the volume of the material, scattering and absorption of the radiation can occur. Scattering is when the radiation incident on an atom does not transfer any energy and is scattered in a different direction. Absorption occurs when the incident radiation has enough energy to cause electrons within that atom to enter an excited state. Both scattering and absorption processes within the material are mathematically described with the Beer Lambert Law,

$$
I=I_{0} \exp \left(-\alpha_{\mathrm{ext}} z\right)
$$

for the transmitted $(I)$ and incident $\left(I_{0}\right)$ intensities, and where the extinction coefficient $\alpha_{\text {ext }}=\alpha_{\text {scat }}+\alpha_{\text {abs }}$ has both a scattering $\left(\alpha_{\text {scat }}\right)$ and absorption component

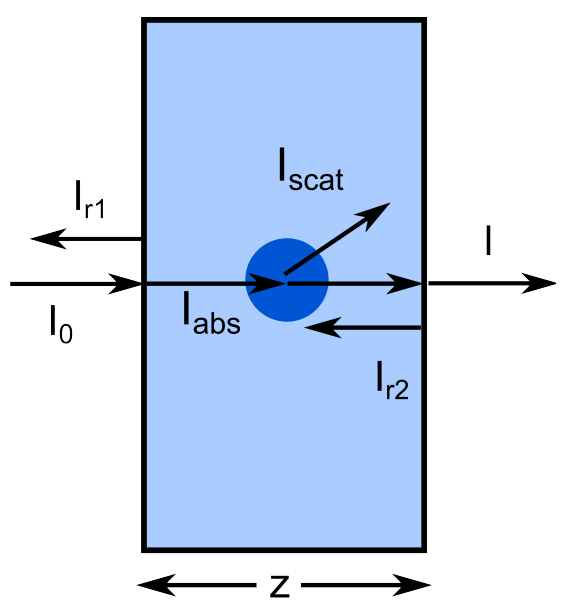

Figure 3.11: Reflection, absorption and scattering of electromagnetic radiation in matter. 
$\left(\alpha_{\mathrm{abs}}\right)$. The total intensity transmitted through a material is,

$$
I=I_{0}(1-R)^{2} \exp (-\alpha z)
$$

which incorporates the losses from each of the three processes. Usually what is measured instrumentally is conventionally labelled the "absorbance" of a material,

$$
A b s=-\log _{10}\left(\frac{I}{I_{0}}\right)=0.4343\left(\alpha_{\mathrm{ext}} z\right)+2 \log _{10}(1+R)
$$

In an optical absorbance measurement the wavelength of radiation incident on the sample is varied and the amount of light transmitted is recorded. To cover the wavelength range $200-800 \mathrm{~nm}$ and $2.5-25 \mu \mathrm{m}$ an ultra-violet visible (UV-Vis) and an infrared (IR) spectrometer were used.

\subsubsection{UV-Vis absorbance}

To measure the absorbance a Shimadzu UV-Vis 2100 spectrophotometer was used, the optics of which are shown in figure 3.12. This spectrophotometer incorporates a dual beam system whereby the incident light beam is split into a sample and a reference beam, and the difference in light intensity is measured. Light is emitted from either a halogen $(\lambda>370 \mathrm{~nm})$ or deuterium $(\lambda<370 \mathrm{~nm})$ lamp and is incident on the monochromator via two mirrors. The monochromator is a Czerny-Turner design which uses a diffraction grating and a double slit system to generate a monochromatic beam. A chopper is used to alternately pass the reference or sample beam into the sample chamber and light from both paths is amplified, collected by a photomultiplier tube and measured with a lock-in amplifier to give the sample absorbance. The spectrophotometer has a wavelength range of $200-900 \mathrm{~nm}$ with a resolution of $0.1 \mathrm{~nm}$ and an absorbance (or optical density) range of $0-5$. 


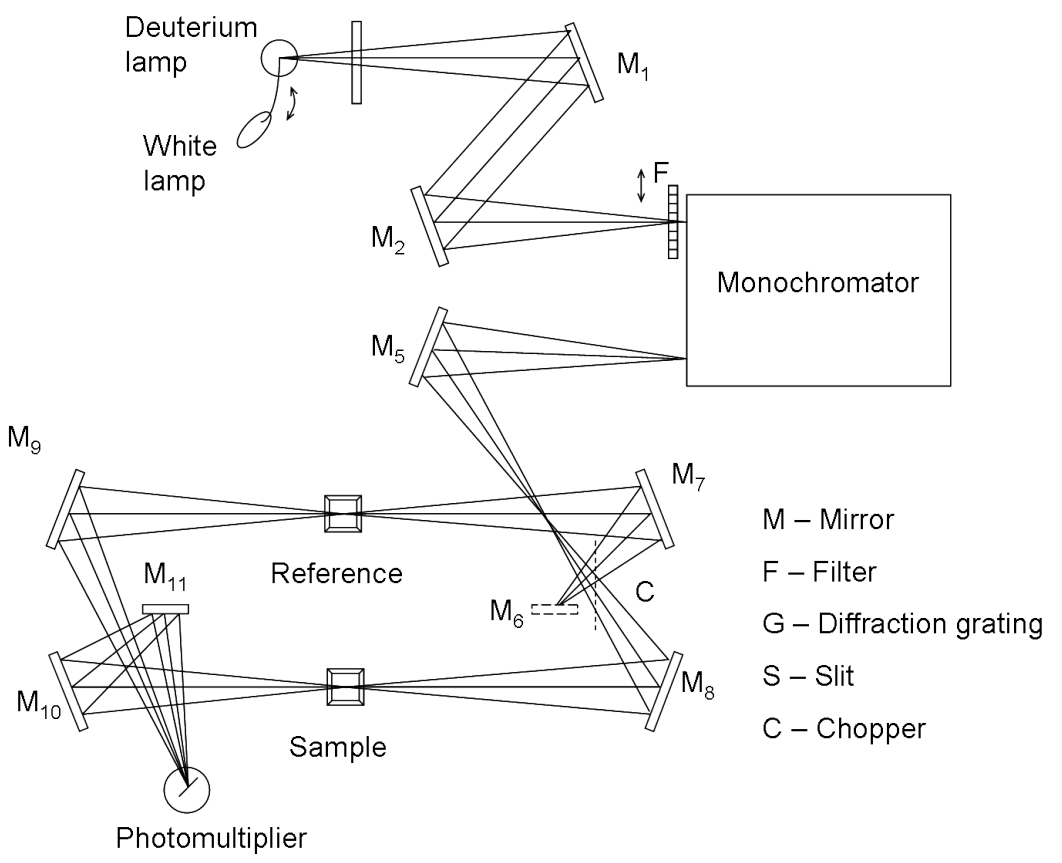

Figure 3.12: Shimadzu UV-Vis 2100 spectrophotometer schematic.

\subsubsection{Infrared spectroscopy}

A Perkin Elmer Spectrum One Fourier transform infrared (FT-IR) spectrometer was used to measure the mid-infrared $\left(450-4000 \mathrm{~cm}^{-1}, 2.5-25 \mu \mathrm{m}\right)$ absorbance of the samples. The spectrometer is based on a Michelson Interferometer, an example of which is shown in figure 3.13 .

Infrared light from the source strikes the beam splitter ( $\mathrm{KBr}$ in this system) where half of the beam is transmitted to the moving mirror and the other half is reflected to the stationary mirror. The moving and stationary mirrors reflect the radiation back to the beam splitter where, again, half is passed to the detector and the other half back to the source. The optical path difference $\delta$ between the two beams from the interferometer is,

$$
\delta=2 d
$$

Firstly, considering a single wavelength (monochromatic light), interference of the recombined beams will occur at the detector. The intensity at the detector is 


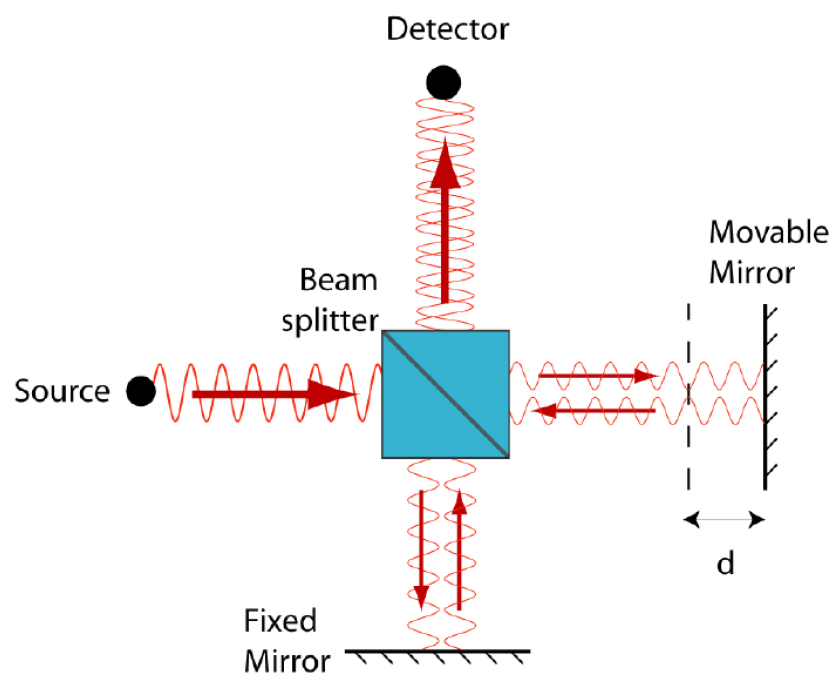

Figure 3.13: Michelson interferometer schematic [46].

given by,

$$
I(\delta)=\frac{I_{0}}{2}\left[1+\cos \left(\frac{2 \pi}{\lambda} \delta\right)\right]
$$

with the maximum intensity occurring at integer multiples of $\lambda$ (ie, $\delta=n \lambda$ ) and minimum at $\delta=(n+1) \lambda$. The variation of intensity with $\delta$ is known as an interferogram.

For polychromatic light, each wavelength can be thought of as producing its own characteristic interferogram (figure 3.14) whose amplitude is weighted by the spectral intensity. The interferogram recorded by the detector is the sum of all the individual wavelength interferograms (red line). The central spike corresponds to zero path difference which is most intense as all wavelengths are simultaneously interfering constructively at this point. The recorded interferogram is then

$$
\begin{aligned}
I(\delta) & =\int_{0}^{\infty} I(\delta, v) d v \\
& =\int_{0}^{\infty} S(v)[1+\cos (2 \pi v \delta)] d v,
\end{aligned}
$$

where $S(v)$ is the spectrum of the light source in wavenumbers $v=1 / \lambda . I(\delta)$ is simply a Fourier cosine transform. In terms of an infrared measurement, the 

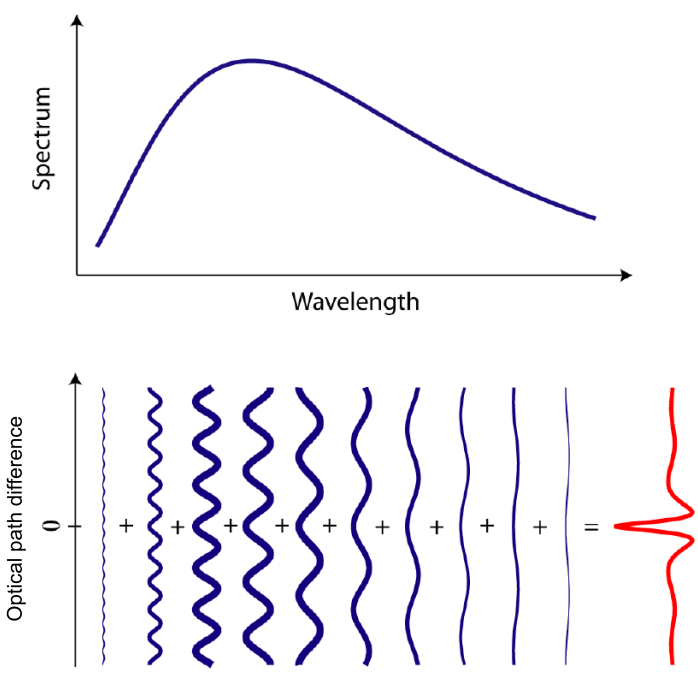

Figure 3.14: Illustration of the formation of a polychromatic light source interferogram [46].

light passing through the sample decreases the magnitude of $I(\delta)$ and $S(v)$ is the absorbance spectrum we are interested in. $S(v)$ can be found by taking the inverse of equation 3.33 - the inverse Fourier cosine transform,

$$
\begin{aligned}
S(v) & =\int_{0}^{\infty} I(\delta) \cos (2 \pi v \delta) d \delta \\
& =4 \int_{0}^{\infty}\left[I(\delta)-\frac{1}{2} I(\delta=0)\right] \cos (2 \pi v \delta) d \delta .
\end{aligned}
$$

FT-IR spectrometers are widely used as they are capable of high resolution; the resolution is simply the inverse of the achievable optical path difference. In this particular spectrometer the resolution is adjustable between 0.5 and $64 \mathrm{~cm}^{-1}$ over a wavelength range of $7800-350 \mathrm{~cm}^{-1}(\sim 1.3-29 \mu \mathrm{m})$.

\subsection{Photoluminescence}

Photoluminescence, as explained in chapter 2.2.1, is a process which can provide information on the electronic structure of a material, particularly dopants within the material. The spectra are collected with a Horiba Jobin Yvon Fluorolog 3 spectrofluorometer. The internal schematics of the specific spectrometer 


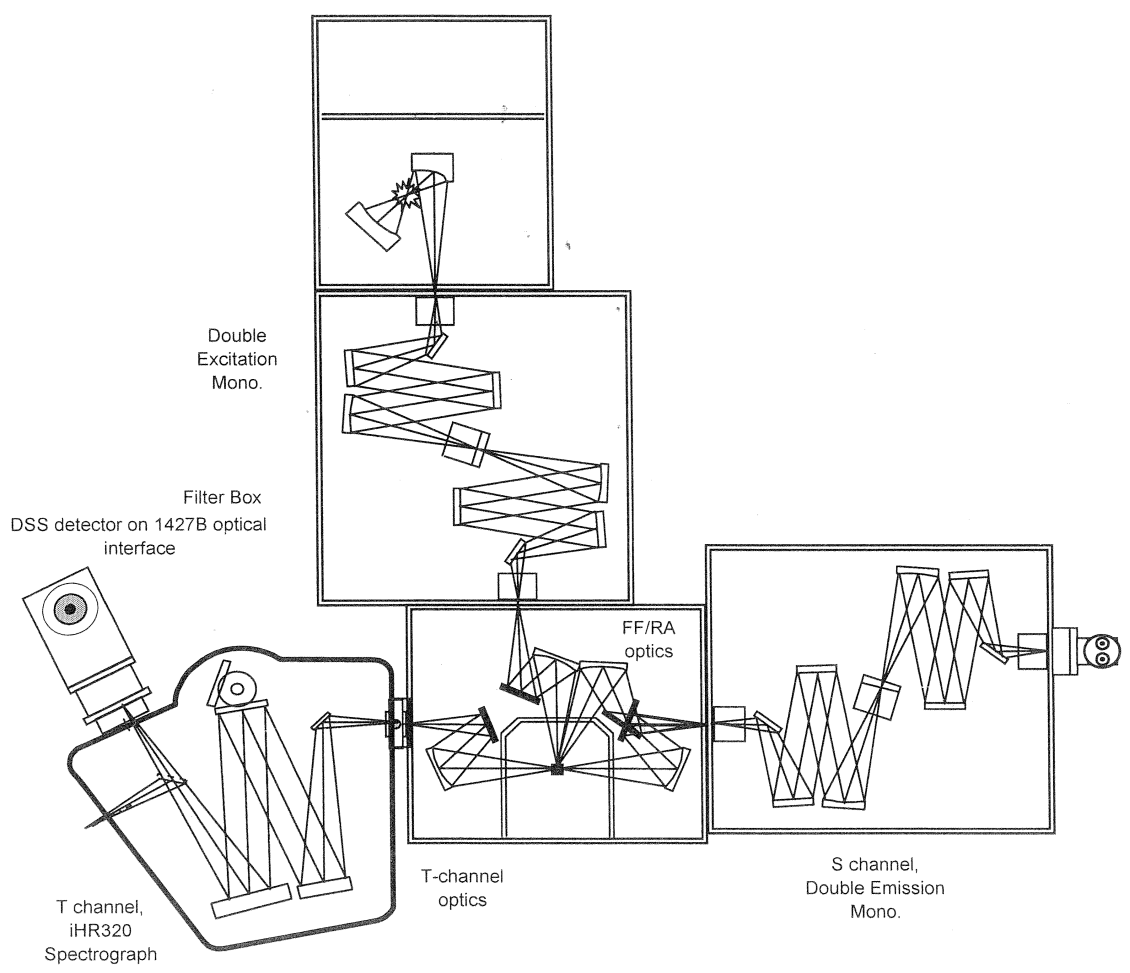

Figure 3.15: Horiba Jobin Yvon Fluorolog 3 schematic.

are shown in figure 3.15. The spectrometer consists of five "modules" - the lamp housing, excitation monochromator, sample chamber and two emission monochromators/detector modules.

The spectrometer operates as follows. Light from a $450 \mathrm{~W}$ xenon arc lamp is incident on the double-grating excitation monochromator which produces the excitation beam. A double monochromator is employed as it offers significant improvement in resolution, sensitivity and stray-light rejection compared to a single monochromator. This particular monochromator has adjustable slits which achieve a bandpass of $0.3-14 \mathrm{~nm}$. The wavelength of the excitation light can range from approximately $200-900 \mathrm{~nm}$. The excitation light passes from the monochromator and is focussed into the sample chamber where it excites the sample and luminescence is emitted. The luminescence is directed toward one of two emission monochromators via rotation of the sample holder.

For ultraviolet-visible luminescence the signal is directed to the right hand detection system on figure 3.15. The luminescence is collected either with front 
face or right angle geometries and is passed through another double monochromator (identical to the excitation monochromator) and then onto a R928P high red-sensitivity photomultiplier tube. This particular photomultiplier operates in a photon counting mode.

Infrared radiation is passed to the left module on figure 3.15, the iHR320 spectrometer. This module consists of a single grating monochromator with interchangeable gratings to allow for a wide range of wavelengths to be scanned. It is capable of detecting infrared wavelengths using GaAs detectors from approximately $800-2500 \mathrm{~nm}$ with a maximum resolution of $0.06 \mathrm{~nm}$ for a 1200 $\mathrm{lp} / \mathrm{mm}$ grating.

A photoluminescence spectrum is produced by stepping either the excitation (excitation spectrum) or emission (emission spectrum) monochromator through a wavelength region and recording the variation in intensity as a function of wavelength. Optical filters can be placed in both the excitation or emission beam. A "SpectrAcq" controller is used to transfer information to and from the spectrometer components and the host computer.

In addition to photoluminescence measurements, photon counting and time resolved spectroscopy can be done using the R928P photomultiplier in the wavelength range $180-850 \mathrm{~nm}$; this is useful for determining photoluminescence lifetimes. For this application high power pulsed LEDs are used to excite the sample and methods such as time correlated single photon counting (TCSPS) or multi-channel averaging (MCA) are used to collect the luminescence as a function of time. LEDs are available with nanosecond pulses (nanoLEDS) or millisecond pulses (spectraLEDs) at a range of wavelengths.

\subsection{Electron paramagnetic resonance}

Electron paramagnetic resonance (EPR) or electron spin resonance (ESR) is a spectroscopic technique for studying chemical species such as ions, free radicals, and dopants with unpaired spins by measuring the absorption of microwaves 
in an applied magnetic field. It is analogous to nuclear magnetic resonance (NMR) except it is the electron spins which are excited rather than the nuclear spins. EPR can yield useful information such as the charge state of a dopant ion, the geometry of an ion or defect site, and the ground state wavefunction character.

A simple system with spin $S=\frac{1}{2}$ and no nuclear interaction is given as an example, the transition diagram of which is shown in figure 3.16(a). The spin Hamiltonian of this system is given by [47],

$$
\mathscr{H}=g \beta_{\mathrm{e}} \mathbf{B} \cdot \mathbf{S}
$$

where $g$ is the electronic $g$-factor, $\beta_{\mathrm{e}}$ the Bohr magneton and $\mathbf{B} \cdot \mathbf{S}$ the dot product between the magnetic field vector and the spin angular momentum. Assuming that the applied magnetic field is parallel to the $\mathrm{z}$ axis equation 3.35 can be written as,

$$
\mathscr{H}=g \beta_{\mathrm{e}} B_{\mathrm{z}} S_{\mathrm{z}}
$$

Transitions between spin states occur when the difference between the spin values $\left(m_{\mathrm{s}}\right)$ is equal to \pm 1 and the energy of the transition is given by,

$$
\Delta E=E_{m_{s}-E_{m_{s}}^{\prime}}
$$

where,

$$
E_{m_{s}}=\left\langle\psi_{m_{s}}|\mathscr{H}| \psi_{m_{s}}\right\rangle=g \beta_{e} B_{z}\left\langle\psi_{m_{s}}\left|S_{z}\right| \psi_{m_{s}}\right\rangle
$$

and,

$$
\left\langle\psi_{m_{s}}\left|S_{z}\right| \psi_{m_{s}^{\prime}}\right\rangle=m_{s}^{\prime} \delta_{m_{s} m_{s}^{\prime}}
$$

In this $S=\frac{1}{2}$ system, one transition occurs between the $m_{s}=1 / 2$ and $m_{s}=$ $-1 / 2$ states with an energy $h \nu=g \beta_{e} B_{z}$. The transitions are induced by the microwave magnetic field which is orientated perpendicular to the applied field. 


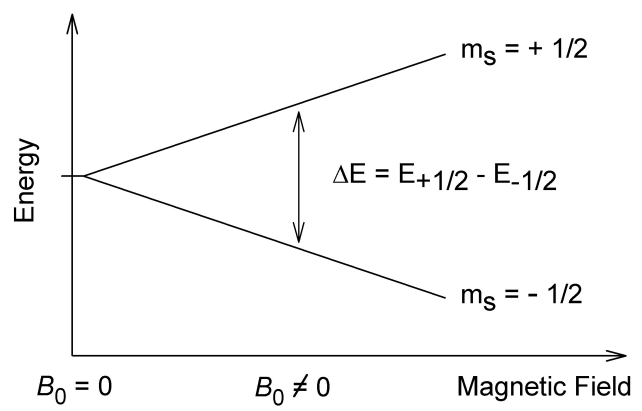

(a)

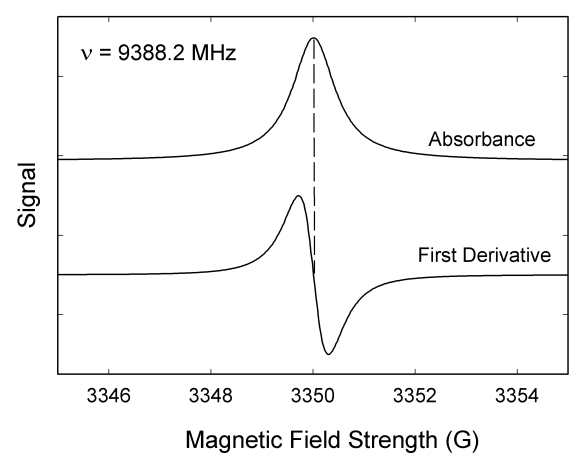

(b)

Figure 3.16: (a) EPR resonance transition and (b) absorbance and first derivative spectrum [48].

An EPR spectrum is experimentally obtained by fixing the microwave frequency and scanning the magnetic field in order to induce electron spin transitions. Therefore, at a magnetic field of,

$$
B_{z}=\frac{h \nu}{g \beta_{e}}
$$

a transition occurs due to absorption of microwaves, and is seen as a peak on figure 3.16(b). Generally, the spectrum recorded is the first derivative of the absorbance (figure $3.16(\mathrm{~b})$ ), and is caused by the oscillating microwaves and lock-in amplifier which are used to improve the signal-to-noise ratio of the system.

The EPR spectra were recorded at X-band ( $\sim 9 \mathrm{GHz})$ using a custom made spectrometer shown in figure 3.16. Microwaves are generated by a Gunn diode which produces an oscillating microwave signal with a frequency between $9-$ 9.5 GHz. The microwaves are first passed through an isolator to stop reflections reaching the Gunn diode and an attenuator is used to adjust the microwave power. The microwaves are then incident on the cavity with the microwave frequency monitored using a frequency counter. The weak reflected signal is separated from the much larger incident power using the properties of a "magic $\mathrm{T}^{\prime \prime}$ component, which splits the power from the Gunn diode equally into arms 2 and 4 . The power in arm 2 is completely absorbed by a matched load whilst 


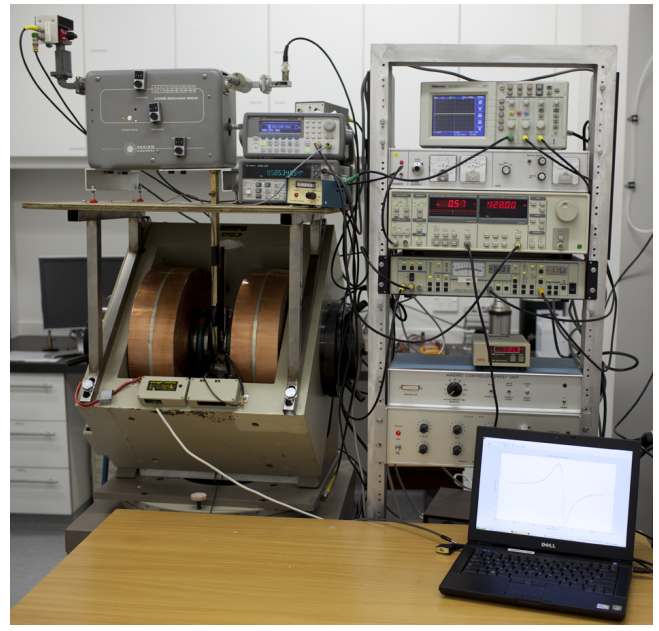

(a)

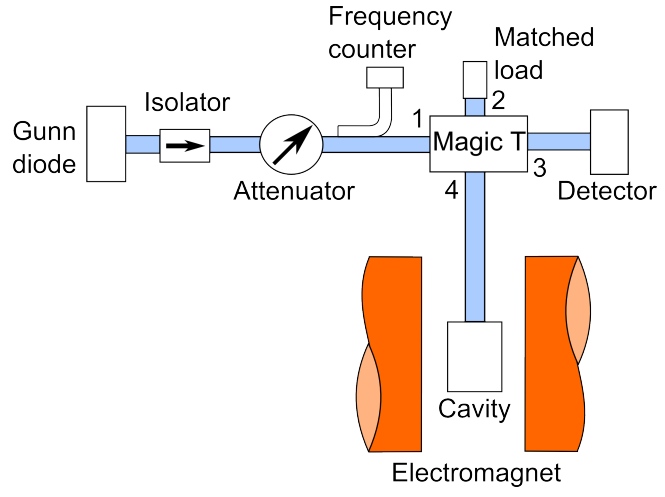

(b)

Figure 3.17: EPR spectrometer (a) photo and (b) schematic.

the signal power reflected from the cavity is split between arms 1 and 3 . A high frequency PN diode in arm 3 detects the EPR signal which has then been separated from the strong microwave power incident on the cavity. The cavity is placed in an electromagnet which produces a $0-600 \mathrm{mT}$ magnetic field. The resonant cavity impedance matching is adjusted so that all the microwaves are absorbed when no sample is present, and no reflection occurs. When a sample is introduced the EPR of the sample in the cavity causes a small signal to be sent back to the detector.

\subsection{Scanning electron microscopy}

A scanning electron microscope (SEM) uses a high energy focused beam of electrons to characterise the morphology, crystalline structure and chemical composition of solid samples. The basic components of a SEM are shown in figure 3.18. The electron gun located at the top of the SEM column produces the electron beam required for SEM measurements. It is either a thermionic gun, which produces electrons by heating a filament, or a field emission gun which uses a strong electric field to pull electrons off atoms by Fowler-Nordheim [49] tunnelling. The electron beam is directed towards the sample by passing 
it through a series of condenser lenses. These lenses are made of magnets which focus and bend the electrons trajectory making them travel in the correct direction. After passing through the condenser lenses the electron beam is incident on the objective lens which focuses the beam to a small spot on the sample. The electron beam is raster scanned across the sample with the use of scanning coils which use a time-varying current to create a magnetic field and manipulate the beam. The size of the sample which is scanned is dependent on the set magnification, the higher the magnification the smaller the scanning area.

When the electron beam strikes the sample, secondary electrons are emitted. These electrons are collected by a secondary electron detector (SEI) whereby the brightness seen on the screen depends on the number of electrons reaching the detector. This gives the general morphology of the sample surface. A back scatter detector can also be used to monitor the number of electrons which are scattered from the surface. This is useful for determining troughs and peaks on the sample surface and for sample composition.

The SEM column must always be under high vacuum due to gases interacting

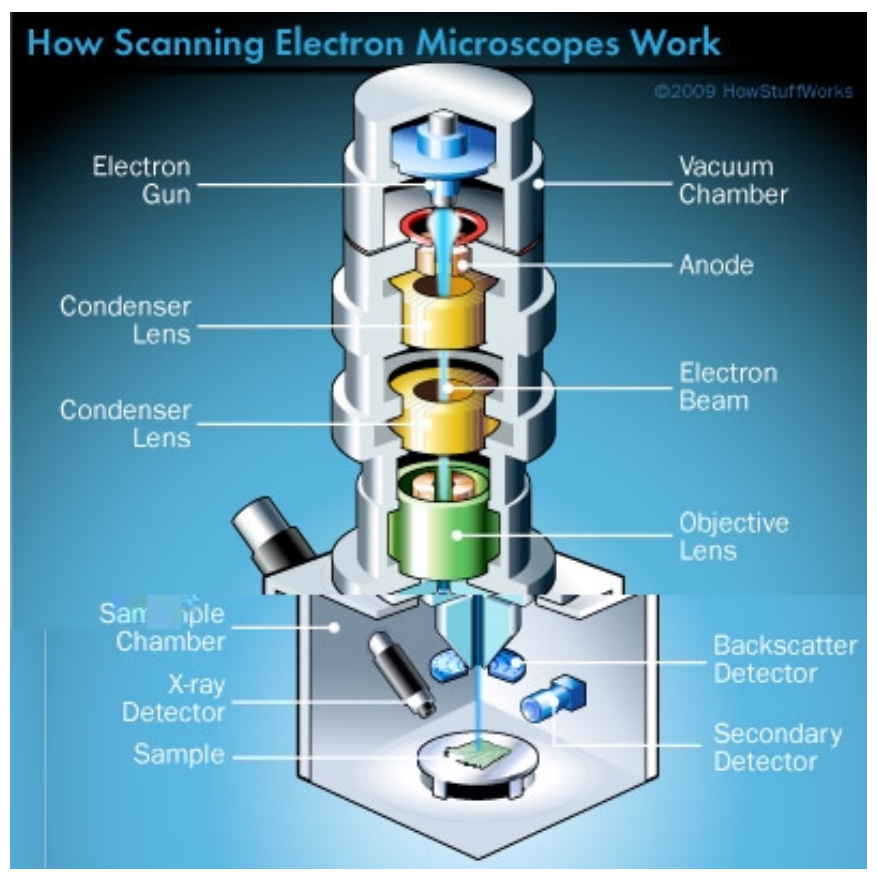

Figure 3.18: Schematic of SEM [49]. 
with and causing instability of the electron beam. This also means that samples must be in a very dry condition before entering the column.

The samples measured in this thesis (chapter 5) are prepared by breaking the sample so the inner surface can be seen and mounting on a right angle stub holder. The sample is coated with an approximately $16 \mathrm{~nm}$ thick carbon layer so that it is electrically conductive and does not charge up in the electron beam. The samples must be placed in a desiccator overnight to remove all water or other solvents which could degrade the vacuum in the column.

The particular SEM used was a JEOL JSM6500F. This SEM uses a field emission gun to produce a $7-8 \mathrm{keV}$ electron beam. Magnifications from $50 x$ to $200,000 x$ are possible depending on the imaging conditions. Typical imaging conditions were $\sim 10 \mathrm{~mm}$ working distance, voltage of $5-10 \mathrm{kV}$ and current of $90 \mu \mathrm{A}$. 



\section{Chapter 4}

\section{Digital camera read-out system}

In developing new storage phosphor materials for X-ray imaging applications it is useful to have the facility to record images and so demonstrate their practical application. A read-out facility is also a valuable tool for determining parameters such as the modulation transfer function which characterise the spatial resolution of any storage phosphor material. As described in chapter 2.2.2. an X-ray image stored in a storage phosphor plate is typically read out by raster scanning a focussed laser beam across the imaging plate. This requires complex and delicate scanning electro-mechanics making the system high in cost; a typical desktop scanner costs in the range of NZ\$50,000 to $\$ 150,000$. To overcome this, a cost-effective imaging system based on a commercially available digital camera and off-the-shelf components was developed [50, 51] which can record images based both on developmental materials and current commercial imaging plates. Until recently this camera-based technique was not viable but dramatic improvements in digital cameras with regards to resolution and low light sensitivity, and the introduction of $\mathrm{CsBr}: \mathrm{Eu}^{2+}$ imaging plates have made it possible. 


\subsection{Technique}

The read-out system using a digital camera is as follows. An X-ray image of an object is generated in the plate or material under test, and the entire plate is then bathed with red stimulating light via high power light emitting diodes. The resulting $\mathrm{X}$-ray image is recorded by a digital camera. Both the camera and LEDs are placed opposite the camera, as demonstrated in figure 4.1 which shows the prototype imaging system built by the author and which implements this technique. Optical filtration in the form of a BG12 blue filter over the camera lens is needed to avoid the stimulating light flooding the camera. The system can be used for imaging plates up to $240 \times 180 \mathrm{~mm}^{2}$ in size.

The main camera used was a Canon EOS 5D MkII digital single lens reflex (SLR) camera equipped with a Canon EF $35 \mathrm{~mm}$ f/1.4L USM wide angle lens. The camera has a full-frame $\left(36 \times 24 \mathrm{~mm}^{2}\right)$ high resolution 21.1 Megapixel CMOS sensor with a pixel size of $6.7 \mu \mathrm{m}^{2}$ and good low light level sensitivity due to its extended ISO range. The ISO setting is essentially a gain control and can be varied in steps from 50 to 25,600; the standard setting for this application is 6400 which is a good balance between high signal and low noise. The f1.4 lens is a

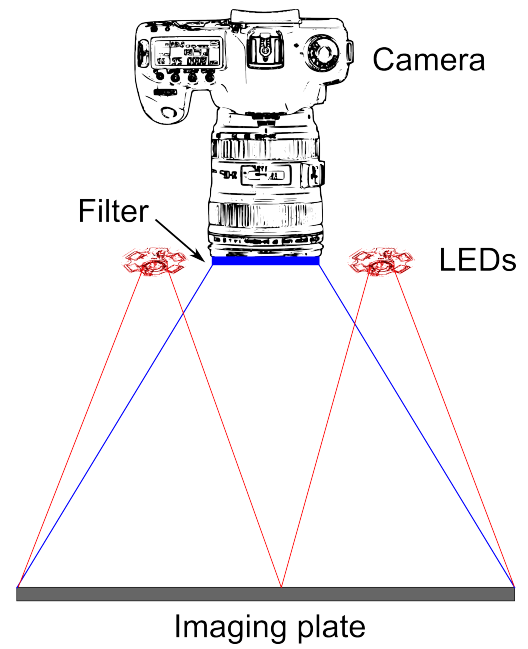

(a)

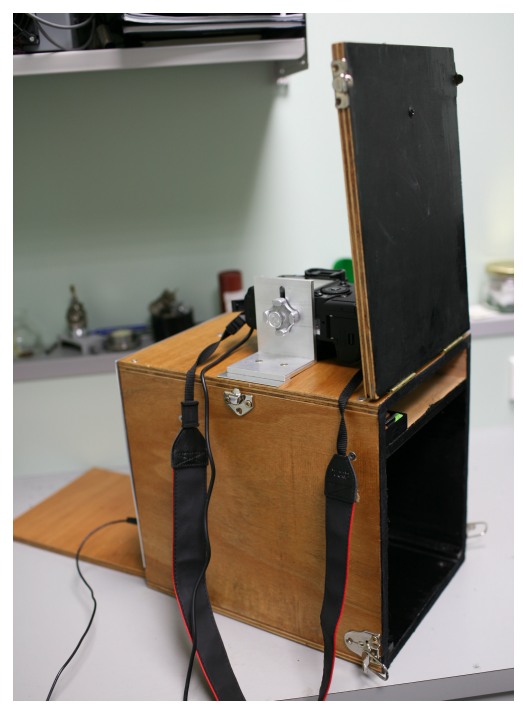

(b)

Figure 4.1: Digital camera imaging system, (a) schematic drawing and (b) prototype. 
high quality wide angle lens with a large aperture which allows for high light throughput hence good low-light sensitivity. The CMOS sensor is followed by a 14 bit analogue to digital converter, and the resulting image is stored in the proprietary Canon CR2 RAW format to preserve as much information as possible. More information on image processing is given in section 4.3 . The camera is placed at an object to sensor distance of $340 \mathrm{~mm}$ and for this distance the image from a standard $240 \times 180 \mathrm{~mm}^{2}$ imaging plate laterally fills the sensor. A second camera based on the competing CCD technology was also tested. This was a SBIG ST-2000XM black and white camera intended for astronomy and was coupled with the same f1.4 lens. The camera has a 1.92 Megapixel Kodak KAI2000M CCD sensor which has sensor dimensions of $11.8 \times 9 \mathrm{~mm}^{2}$ and a pixel size of $7.3 \mu \mathrm{m}^{2}$. It has a 16 bit analogue to digital convertor and the images are saved as a 16 bit TIFF. A field of view of $83 \times 62 \mathrm{~mm}^{2}$ was achieved at a distance of $340 \mathrm{~mm}$. This CCD camera also has a built in cooling system which is used to decrease the thermal noise level in the sensor.

For testing purposes two imaging plates were used, a Konica Minolta CP1M200 $\mathrm{CsBr}: \mathrm{Eu}^{2+}$ needle imaging plate intended for mammographic imaging, and a Fuji ST-VI BaFBr:Eu ${ }^{2+}$ general purpose radiography imaging plate.

The stimulating light-emitting diodes (LEDs) are placed opposite the imaging plate and high enough to cover the entire plate. The LEDs used are high intensity Philips Lumileds Luxeon III Star LEDs which are capable of fast readout and hence low sensor noise. The LEDs are powered with a custom timer circuit (shown in appendix A) which allows multiple colours, two to four LEDs on at a time, pre-selected stimulation time and synchronisation with the camera shutter (only for the Canon SLR). Colours include red $(627 \mathrm{~nm})$ for stimulation, blue $(470 \mathrm{~nm})$ for focussing and white for photographic imaging capabilities. Four red stimulation LEDs provide reasonably uniform illumination across the plate.

Filtration of the stimulating light and transmission of the emission light is 
achieved with a Schott BG12 filter. This is required as the stimulation light is many orders of magnitude greater than the emission light and can flood the camera's sensor.

Focussing of the two cameras was initially achieved manually using the "live" mode on the Canon camera and the focussing option on the CCD camera. An X-ray image of an object with sharp lines was stored in the imaging plate and was slowly read-out using short low intensity stimulation light pulses. The cameras were focussed onto this image using the manual focussing ring of the lens. A focusing aid was made for the Canon camera based on the position found by this method. It was found that the focusing of the image on the $\mathrm{CsBr}: \mathrm{Eu}^{2+}$ imaging plate occurs at the surface whereas the $\mathrm{BaFBr}: \mathrm{Eu}^{2+}$ imaging plate occurred approximately $8 \mathrm{~mm}$ above the surface of the plate. The reason for this is unclear, but may be a consequence of chromatic aberration in the lens. For the CCD camera focusing of the image was at the surface for both imaging plates.

With digital cameras it is possible for photographic images of the same object being X-rayed to be captured, an additional feature of the prototype system. Registration of the two images will show where features seen on the X-ray image can be found in the object.

\subsection{Spectral sensitivity}

A CCD or CMOS silicon sensor has no inherent colour sensitivity in the visible region, but is made colour sensitive by a filter arrangement in the form of organic dyes on the camera sensor. For the Canon camera a Bayer pattern, such as that shown in figure 4.2 , is used where in a two-by-two grid of pixels the colour pattern is red-green-green-blue. The CCD camera does not have any colour filters.

The sensitivity of the CMOS camera to wavelength for each of the three colour channels (figure 4.3(a)) was measured by recording images of different wave- 


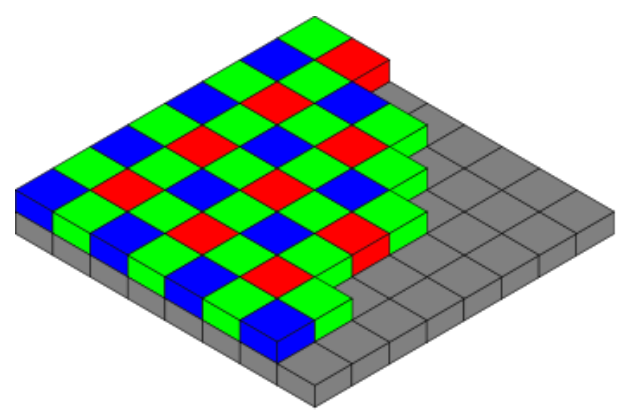

Figure 4.2: Bayer filter pattern used on the Canon 5D MkII [52].

lengths of light produced by a spectrometer based around a xenon lamp and double monochromator (Horiba Jobin Yvon Fluorolog 3). For each image the intensities of each colour channel were pixel-averaged separately and then calibrated for intensity with an Ocean Optics USB4000 CCD spectrometer. As can be seen in figure 4.3(a) the maximum sensitivity occurs at 600, 530 and 450 $\mathrm{nm}$ for the red, green and blue channel respectively. The luminescence emission for current commercial imaging plates, shown in figure $4.3(\mathrm{~b})$, is $440 \mathrm{~nm}$ for $\mathrm{CsBr}: \mathrm{Eu}^{2+}$ and $390 \mathrm{~nm}$ for $\mathrm{BaFBr}: \mathrm{Eu}^{2+}$ which corresponds to the cameras blue channel. Therefore, the best matched imaging plate is one based on $\mathrm{CsBr}: \mathrm{Eu}^{2+}$ as its emission occurs at the peak of the blue channel, whereas only the tail end of the emission from the BaFBr: $\mathrm{Eu}^{2+}$ will be captured.

The filtration of stimulation light is achieved using a $3 \mathrm{~mm}$ thick Schott BG12 filter, the transmission characteristics of which can be seen in figure 4.3(c). This filter clearly blocks the stimulating light and is suitable for transmitting the emission light for both types of imaging plate. An alternative filter which is often used (and more readily available) is a BG3 filter. This filter shows improved transmission in the blue band but is inferior in the red stop band, causing leakage of the stimulation light into the sensor. 


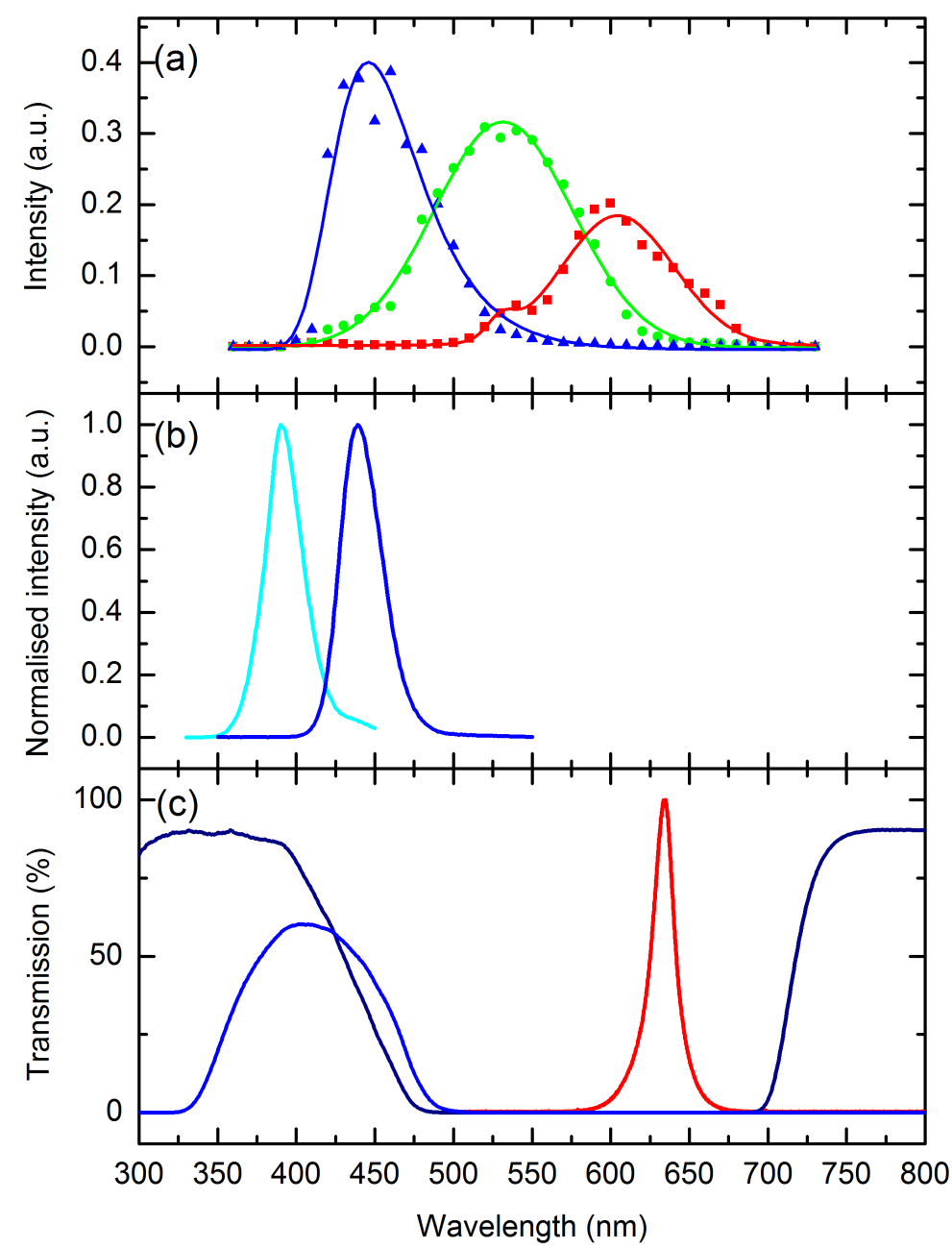

Figure 4.3: (a) Spectral sensitivity of the camera sensor's blue (triangles), green (circles) and red (squares) channels, (b) PSL emission of CsBr:Eu+ (blue line) and BaFBr:Eu ${ }^{2+}$ (cyan line), and (c) stimulating LED (red line) and BG12 (blue line), BG3 (navy line) filter characteristics.

\subsection{Image processing}

Images from the Canon camera are recorded in RAW format for which the detailed file format is not publicly available. The RAW format is converted into a workable format (TIFF) using a freely available software program called "dcraw" [53]. The image is converted into TIFF format via the commands in table 4.1 which leaves the image simply as the raw intensity values from each pixel. To extract the blue channel image the resultant TIFF image is opened in MATLAB and every fourth pixel in a $2 \times 2$ array is stored as the blue channel image. The image can then be saved or further processed using MATLAB 
Table 4.1: dcraw commands for converting Canon CR2 files to a TIFF with raw pixel values [53].

\begin{tabular}{cc}
\hline Command & Description \\
\hline$-\mathrm{D}$ & Document mode without scaling (totally raw) \\
-4 & 16 bit linear \\
$-\mathrm{T}$ & Write TIFF image \\
$-\mathrm{j}$ & Don't stretch or rotate raw pixels \\
$-\mathrm{t} 0$ & No image rotation \\
\hline
\end{tabular}

or other software. Other channel images can also be created by selecting the relevant colour pixels.

The CCD camera software directly saves the resultant image as a 16 bit TIFF file and any subsequent image processing can be applied directly.

\subsection{Spatial resolution}

The optical spatial resolution of both cameras was measured using the sinusoidal variation on a Koren lens test chart [54] (figure 4.4(a)] placed at the same distance used for X-ray imaging. The modulation transfer function (MTF) is found by taking a line across the sinusoidal variation and calculating the difference between the peaks and troughs for each spatial frequency.

The X-ray resolution of the imaging system was measured using a Nuclear Associates grating phantom (type 07-553, 0.25-10 lp/mm), based on a lead grid, and shown in figure 4.4(b), An X-ray image of the test grid is captured and a line perpendicular to the grid is obtained. The contrast function for each spatial frequency is least-squares fitted with a sinusoidal function of which the fitted amplitude is used to determine the MTF.

The optical MTF for the CMOS camera is shown as the black line in figure 4.5(a). The spatial frequencies refer to the imaging plate and not the sensor, with a sensor pixel size of $6.4 \mu \mathrm{m}^{2}\left(6.4 \mu \mathrm{m}^{2}\right.$ indicates a pixel size of $6.4 \mu \mathrm{m} \mathrm{x}$ $6.4 \mu \mathrm{m})$ corresponding to an effective image pixel size of $44 \mu \mathrm{m}^{2}$. However, the maximum possible resolution is decreased due to the colour filter arrangement 


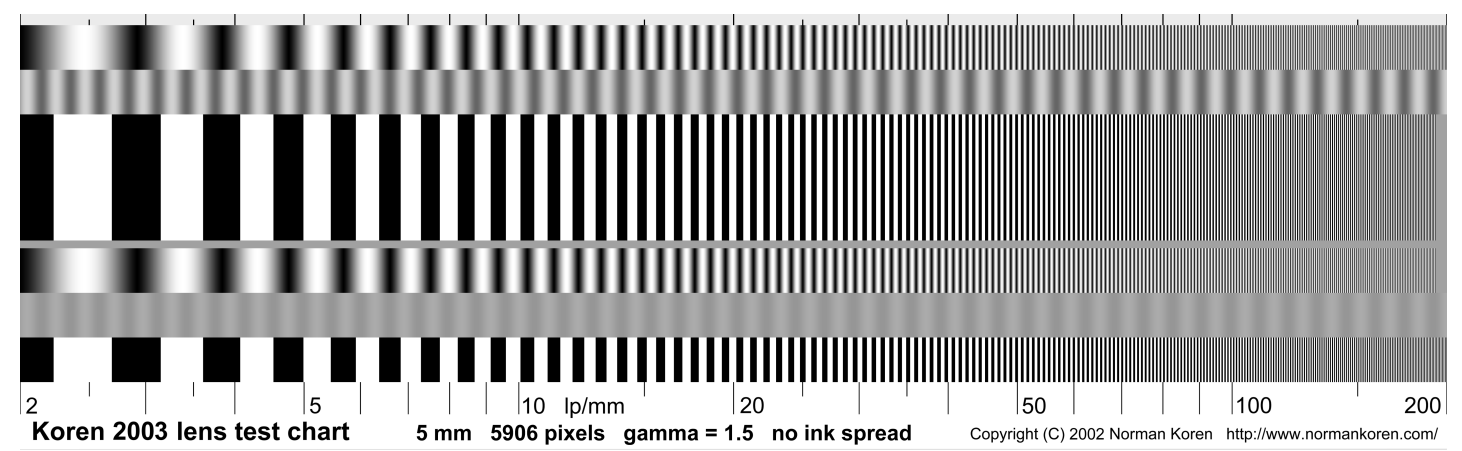

(a)

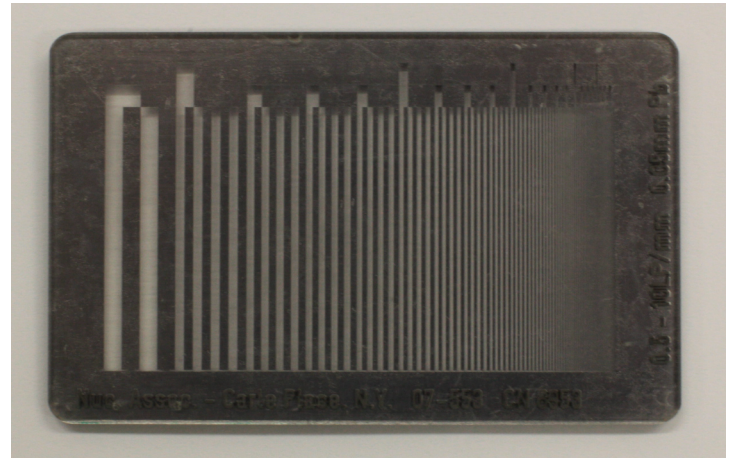

(b)

Figure 4.4: (a) Koren lens test chart [54] and (b) Nuclear Associates grating phantom used for resolution measurements. Note that the Koren chart contains both square wave and sinusoidal wave patterns.

on the sensor. One in four pixels is blue which increases the image pixel size to $88 \mu \mathrm{m}^{2}$. An ideal MTF, limited only by the pixel binning, would therefore pass through zero at a frequency of around $11.4 \mathrm{lp} / \mathrm{mm}$ with a classic sinc shape (see appendix B). The measured MTF is not in good agreement with this, as the theory above does not include the effect on resolution of the lens system.

The X-ray MTFs for both the $\mathrm{CsBr}: \mathrm{Eu}^{2+}$ and $\mathrm{BaFBr}: \mathrm{Eu}^{2+}$ imaging plates are also shown in figure 4.5(a), The data points have been least squares fitted with the sum of two Gaussians. The resolution at an MTF of 0.2 is around $2.2 \mathrm{lp} / \mathrm{mm}$ for $\mathrm{CsBr}: \mathrm{Eu}^{2+}$ and $1.6 \mathrm{lp} / \mathrm{mm}$ for $\mathrm{BaFBr}: \mathrm{Eu}^{2+}$, compared to the claimed values for the commercial imaging plates of 3.5 and $2.5 \mathrm{lp} / \mathrm{mm}$ for $\mathrm{CsBr}_{\mathrm{Eu}}{ }^{2+}$ and BaFBr:Eu ${ }^{2+}$ respectively [11]. The commercial scanning systems clearly show better resolution than achieved here, probably due to the scanning pixel size set by the laser optics being smaller than our pixel size at the imaging plate. The $\mathrm{X}$ - 


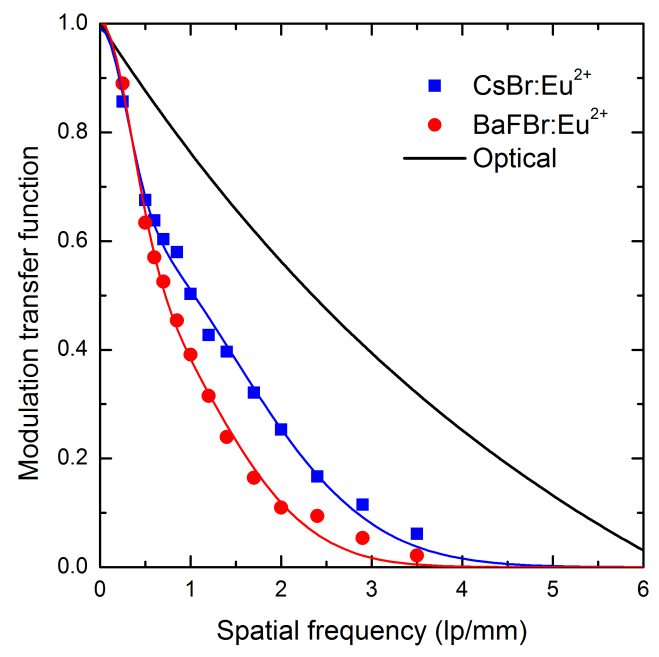

(a)

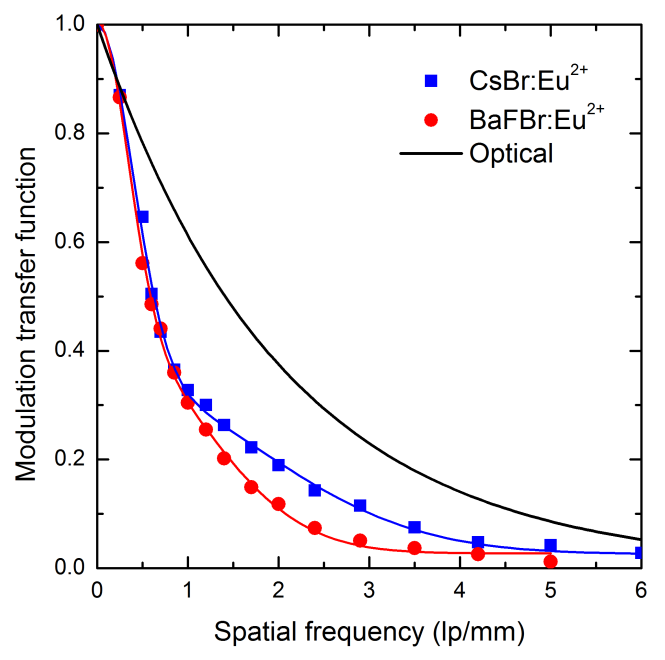

(b)

Figure 4.5: Optical (upper line) and X-ray MTFs (lower lines, blue squares CsBr: $\mathrm{Eu}^{2+}$, red circles BaFBr:Eu ${ }^{2+}$ ) obtained by the grating method for (a) the CMOS based system and (b) the CCD based system. The fitted lines are the sum of two Gaussians.

ray MTFs are inferior to that of the optics alone which suggests that the limiting factor in this system is the imaging plates themselves, not the optics. This can be explained by light scattering of the emission (PSL) light in the imaging plate causing the image to diffuse within the plate. The extent of the lateral diffusion from a line image in the plate depends upon the depth of the image. An X-ray image generated closer to the surface of the $\mathrm{CsBr}: \mathrm{Eu}^{2+}$ imaging plate (by using a lower tube voltage) has a resolution of $3 \mathrm{lp} / \mathrm{mm}$ at a MTF of 0.2 supporting the explanation. In a shallower image the scattered PSL light originates from a smaller surface area than for a deeper image. The slightly better resolution for the $\mathrm{CsBr}: \mathrm{Eu}^{2+}$ imaging plate can be attributed to the guiding of PSL light generated within a $\mathrm{CsBr}$ needle.

Similarly the CCD optical and X-ray MTFs are shown in figure 4.5(b), The CCD sensor has a pixel size of $7.3 \mu \mathrm{m}^{2}$ corresponding to an image pixel size of 52 $\mu \mathrm{m}^{2}$. The theoretical limit of the optical MTF is $19.2 \mathrm{lp} / \mathrm{mm}$ with a sinc shape, which is clearly not reached in this system. The MTF appears to be slightly different to that for the CMOS camera, which may be a result of different optical scattering processes in the two different sensors and their overcoatings. The 
X-ray MTFs have again been fitted with a sum of two Gaussians. At an MTF of 0.2 the resolution is 2.0 and $1.5 \mathrm{lp} / \mathrm{mm}$ for the $\mathrm{CsBr}: \mathrm{Eu}^{2+}$ and $\mathrm{BaFBr}: \mathrm{Eu}^{2+}$ plates respectively; these values are comparable to the CMOS camera as expected if it is the plates themselves, not the camera, which primarily sets the resolution.

\subsection{Sensitivity to X-ray dose}

The sensitivity of the imaging system to X-ray dose is important as it determines the types of applications it can be used for, e.g. for medical imaging the dose for an adequate image must be less than approximately $0.1 \mathrm{mSv}$. This was determined by measuring the pixel count (for the blue channel) of the two plate types when irradiated with the dental X-ray set for various exposure times. The dose was measured using a Thermo Scientific personal dosimeter.

Figure 4.6(a) shows the response of the CMOS camera system to X-ray dose for the $\mathrm{CsBr}: \mathrm{Eu}^{2+}$ and BaFBr:Eu${ }^{2+}$ imaging plates. The apparent sensitivity for the $\mathrm{CsBr}: \mathrm{Eu}^{2+}$ plate is approximately four times higher than for $\mathrm{BaFBr}: \mathrm{Eu}^{2+}$ which is possibly due to two factors, (i) higher attenuation of the BaFBr:Eu ${ }^{2+}$ PSL light by the blue pixel dye (figure 4.3(a) and (b)), and (ii) a higher basic sensitivity for the CsBr:Eu${ }^{2+}$ plate.

The response of the CCD camera based imaging system to X-ray dose for both imaging plates is shown in figure $4.6(\mathrm{~b})$. Here the sensitivity is much the same for both plates suggesting that the main difference in sensitivities for the CMOS camera shown in figure $4.6(\mathrm{a})$ is indeed due to the sensor dye filtration. In the range approximately 10 to $10,000 \mu \mathrm{Sv}$ the profile is linear, with the deviation at doses lower than $10 \mu \mathrm{Sv}$ being attributed to the noise floor inherent in the read-out process. The CCD camera shows a large dynamic range of around three orders of magnitude which is important for applications which require low contrast imaging. 


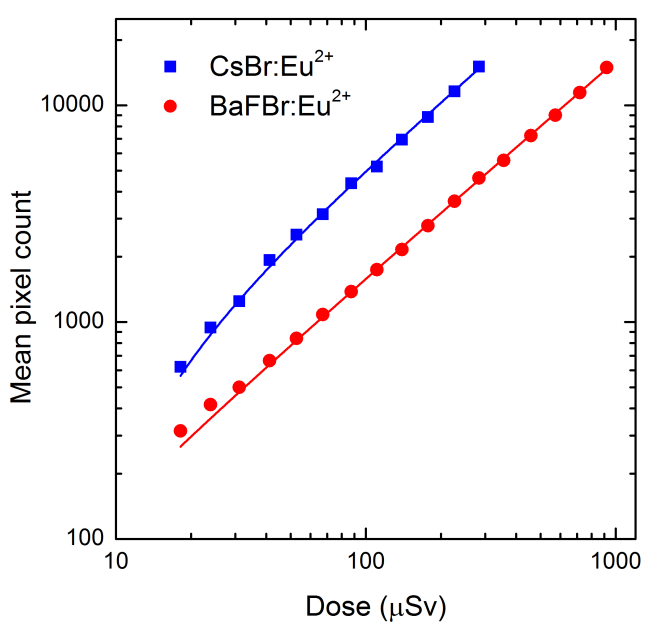

(a)

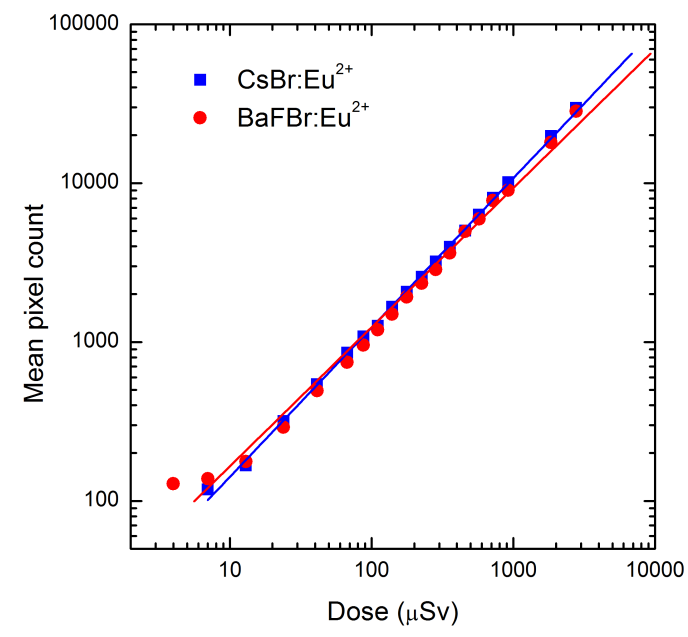

(b)

Figure 4.6: Mean pixel count versus X-ray dose for $\mathrm{CsBr}: \mathrm{Eu}^{2+}$ (blue squares) and $\mathrm{BaFBr}: \mathrm{Eu}^{2+}$ (red circles) imaging plates for the (a) CMOS based system and (b) CCD based system.

\subsection{Read-out properties}

The dark count and its standard deviation give an indication of the lowest workable pixel count and the amount of noise in the image. To achieve a dark image with the CMOS camera the lens cap was left on and an image was captured using the same settings as for an X-ray image. The mean dark count and associated standard deviation for various exposure times as a function of full scale is shown in figure 4.7(a); full scale is a pixel value of 16,383 . The typical dark count is 1030 with a standard deviation of 40 . Increasing the exposure time increases both the dark count and standard deviation with a corner time of around $2 \mathrm{~s}$, above which the noise increases.

The CCD camera has a built in feature to capture (and subtract) dark images which involves keeping the internal shutter closed. Additionally the cooling feature of the camera helps to reduce the image noise. At the typical operating temperature of $-10{ }^{\circ} \mathrm{C}$ the mean dark count is around 1080 with a standard deviation of 19; full scale with this sensor is 65,535 . Figure $4.7(\mathrm{~b})$ shows the mean dark count and standard deviation for various exposure times. The mean count actually decreases slightly above exposure times of $1 \mathrm{~s}$ whereas the standard 


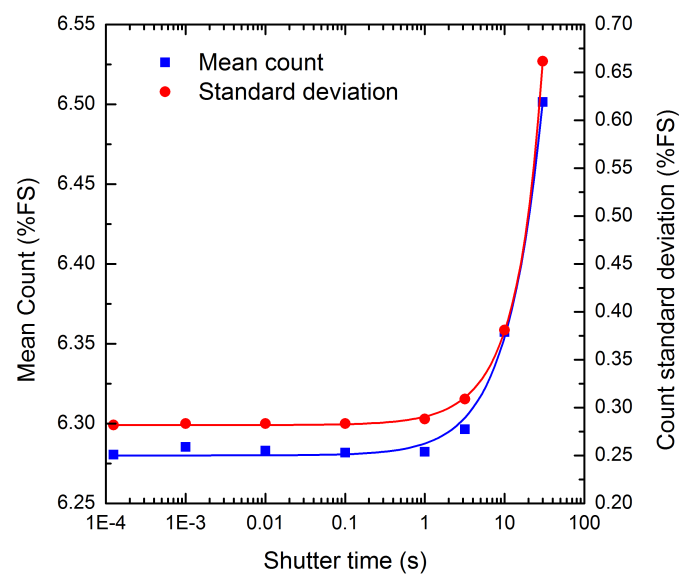

(a)

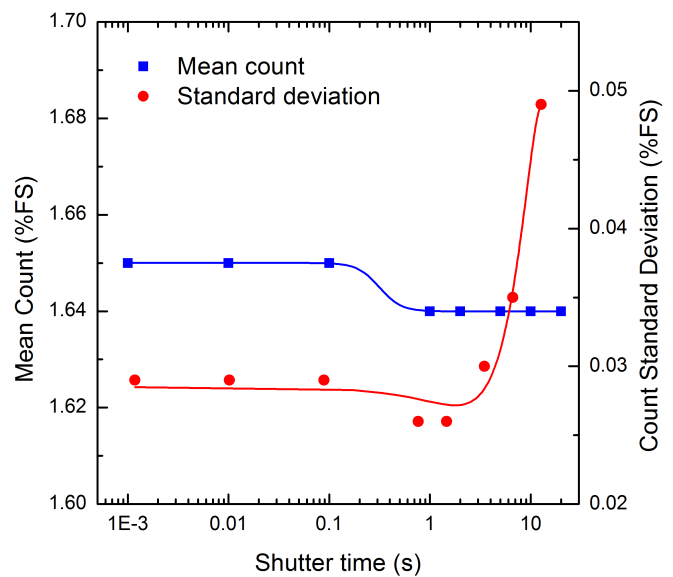

(b)

Figure 4.7: Mean dark count per pixel (blue squares) and standard deviation (red circles) as a percentage of full scale versus shutter time for the (a) CMOS based system and (b) CCD based system.

deviation increases at times above $2 \mathrm{~s}$, for reasons which are probably associated with the internal read-out electronics. Therefore for both the CMOS and CCD based systems the exposure time should be kept below $2 \mathrm{~s}$ to minimise the noise in the images. This places strong demands on the LED intensity as a high fraction of the image needs to be read out in this time.

The fraction of the PSL image read out with successive light pulses was measured (figure 4.8) to determine the read-out time. For the CMOS camera the shutter is directly linked to the start of the LED pulse and $200 \mathrm{~ms}$ LED pulses along with 200 ms shutter times were used. With the CCD camera this is slightly more complicated as the actual start time of the exposure is difficult to determine. To overcome this, a shutter time of $2 \mathrm{~s}$ was used and the LED was pulsed on for $200 \mathrm{~ms}$ in the middle of the shutter period. The curves show that for both systems a shutter time of $2 \mathrm{~s}$ is sufficient to read out a large fraction of the PSL image $\left(95 \%\right.$ and $99 \%$ for the $\mathrm{CsBr}: \mathrm{Eu}^{2+}$ imaging plate read out with the CMOS and CCD based systems respectively).

The read-out curves for each imaging plate (figure 4.8) are expected to be similar for both camera systems. The amount of PSL intensity read out as a function of time is given by,

$$
I=I_{0}(1-\exp (-t / \tau))
$$




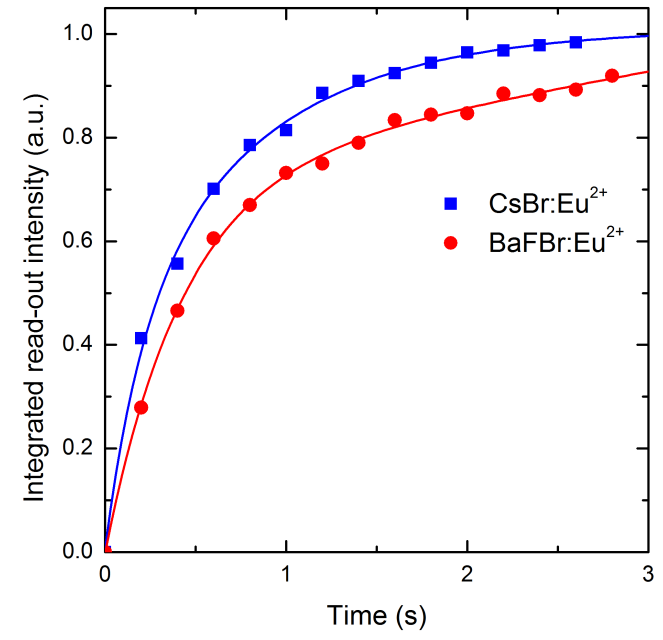

(a)

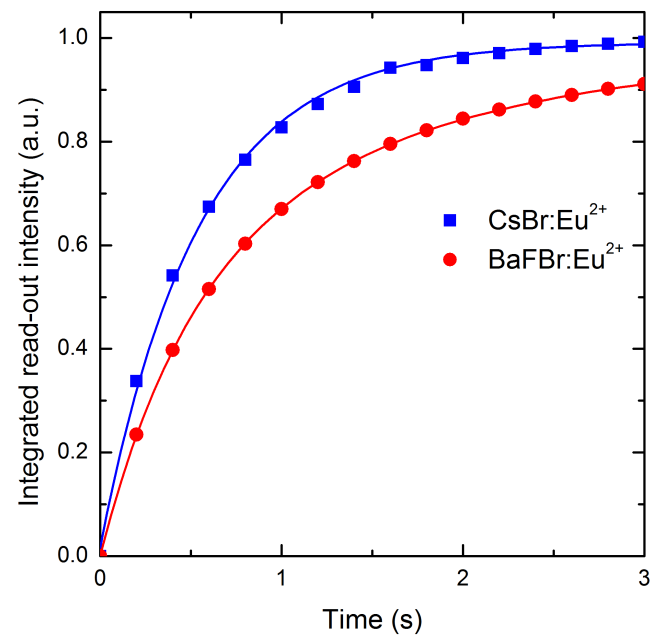

(b)

Figure 4.8: Fraction of image read out following successive $200 \mathrm{~ms}$ pulses of LED illumination for $\mathrm{CsBr}: \mathrm{Eu}^{2+}$ (blue squares) and $\mathrm{BaFBr}: \mathrm{Eu}^{2+}$ (red circles) imaging plates for the (a) CMOS based system and (b) CCD based system.

The intensity of the stimulation light $\left(I_{0}\right)$ is fixed and does not change between the imaging plates or camera system (same stimulating light is used for each system). Therefore, the shape of the read-out curve is given by the decay constant $\tau$, which for a given imaging plate, should be the same for both cameras. The decay constants for the $\mathrm{CsBr}: \mathrm{Eu}^{2+}$ imaging plate were found to be comparable at 0.54 and $0.56 \mathrm{~s}$ for the CMOS and CCD camera systems respectively. For the $\mathrm{BaFBr}: \mathrm{Eu}^{2+}$ imaging plate, decay constants of 0.89 and 0.98 $\mathrm{s}$ were found for the CMOS and CCD camera systems respectively.

\subsection{Detective quantum efficiency}

The detective quantum efficiency (DQE) is an important performance factor for imaging systems since it can be used to compare the achievable signal-to-noise ratio of different systems. It is defined as the ratio of the square of the signal-tonoise at the output of the given system to the square of the signal-to-noise ratio of the input,

$$
\operatorname{DQE}(\nu)=\frac{S N R(\nu)_{\text {output }}^{2}}{S N R(\nu)_{\text {input }}^{2}}
$$


and is ideally one for all frequencies $\nu$.

An americium-241 radioactive source was used to produce an X-ray image with a known photon count as it produces mainly $59.5 \mathrm{keV}$ X-ray photons at a rate of $\sim 1.3 \times 10^{9}$ photons per second. The X-ray photon count rate was calculated assuming only $59.5 \mathrm{keV}$ photons escaped through the encapsulation; attenuation of the $59.5 \mathrm{keV}$ photons by the encapsulation was estimated by measuring the actual dose rate and comparing with that calculated using the known activity (3.4 GBq) (see section 3.3.1). The input signal is the number of photons $(Q)$ arriving over a given area at a set distance from the source. As X-ray photons follow Poisson statistics the signal noise is $\sqrt{Q}$ and the overall signal-to-noise ratio of the input is $Q / \sqrt{Q}=\sqrt{Q}$. The signal, $S$, produced by the computed radiography system is reduced by the MTF, and the DQE in equation 4.2 can be determined by [20],

$$
\operatorname{DQE}(\nu)=\frac{S^{2} M T F(\nu)^{2}}{Q N P S(\nu)}
$$

where NPS is the noise power spectrum of the output.

The noise power spectrum is defined as the variance of the image noise divided among the frequency components of a flat field image, and is defined in two dimensions as [55],

$$
\operatorname{NPS}\left(\mu_{n}, \nu_{k}\right)=\lim _{N_{x} N_{y} \rightarrow \infty} \lim _{M \rightarrow \infty} \frac{N_{x} N_{y} \Delta x \Delta y}{M} \sum_{m=1}^{M}\left|F T_{n k}[I(x, y)-\bar{I}]\right|^{2},
$$

where $I(x, y)$ is the image pixel intensity, $\bar{I}$ is the global mean intensity, $\mu$ and $\nu$ are the spatial frequencies for $x$ and $y, N_{x}$ and $N_{y}$ are the number of pixels in the $x$ and $y$ direction, $\Delta x$ and $\Delta y$ are the pixel spacings and $M$ is the number of regions used for analysis. FT refers to the Fourier transform of the pixel intensities. The process of determining the NPS of a given X-ray image is implemented using MATLAB and is as follows. A region of 256 x 256 pixels from the centre of the flat field image is chosen and the intensities are least- 
squares fitted with a polynomial of order two (in both $x$ and $y$ directions). The fitting is subtracted to compensate for the non uniform illumination of the plate, and this removes the need to subtract the global mean $(\bar{I})$ in equation 4.4. Each row of $256 \times 1$ pixels could be used to generate a one dimensional NPS, analogous to the two dimensional version in equation 4.4 . However, each row would give a different result and so some averaging process is called for. The region of interest is split into sub regions of 8 rows, with each sub region having a common 4 rows from the previous sub region, making a total $(M)$ of $63 \mathrm{sub}$ regions. For each sub region the rows are averaged, Fourier transformed using the fast Fourier transform (FFT) algorithm, and the resulting row values are squared. This is done for each region and averaged, the resulting row is multiplied by the pixel spacings to give the NPS in one dimension. The prefactor of $N_{x} N_{y}$ in equation 4.4 does not need to be applied for the MATLAB FFT implementation.

Figure 4.9 shows the DQE for both the CMOS and CCD camera systems. The CMOS camera based system has a maximum DQE of 0.18 for $\mathrm{CsBr} \mathrm{Eu}^{2+}$ and 0.17 for $\mathrm{BaFBr}: \mathrm{Eu}^{2+}$ and is lower than reported for laser-based read-out systems, 0.55 for $\mathrm{CsBr}: \mathrm{Eu}^{2+}$ and 0.25 for $\mathrm{BaFBr}: \mathrm{Eu}^{2+}$ [11, 20]. This can be attributed

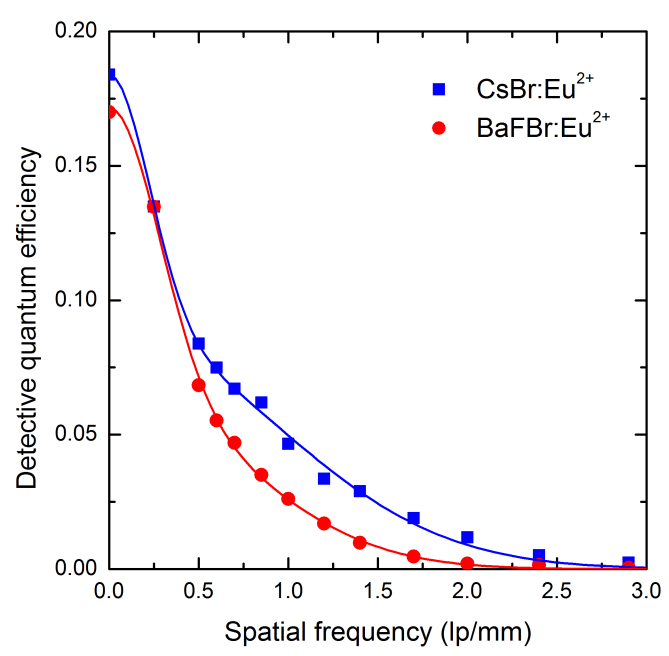

(a)

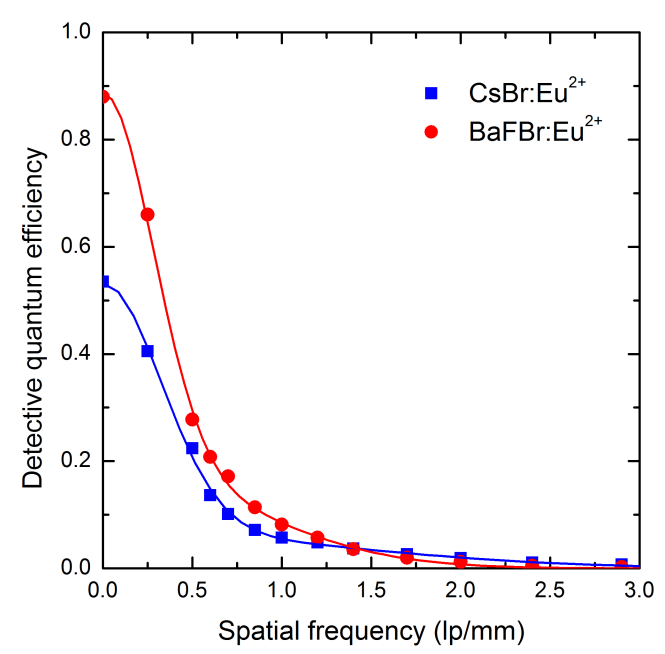

(b)

Figure 4.9: DQE for CsBr:Eu ${ }^{2+}$ (blue squares) and BaFBr:Eu ${ }^{2+}$ (red circles) imaging plates for the (a) CMOS based system and (b) CCD based system. The fitted lines are the sum of two Gaussians. 
Table 4.2: Percentage absorption in $\mathrm{BaFBr}$ and $\mathrm{CsBr}$ for various energies. Note the increase in absorption is due to absorption edges (see chapter 2.1.1).

\begin{tabular}{ccc}
\hline Energy $(\mathrm{keV})$ & BaFBr $(\%)$ & $\mathrm{CsBr}(\%)$ \\
\hline 10 & 100 & 100 \\
20 & 99.8 & 96.4 \\
30 & 87.9 & 67.4 \\
40 & 99.5 & 93.9 \\
50 & 94.7 & 79.2 \\
60 & 83.6 & 61.8 \\
\hline
\end{tabular}

to the high noise characteristics of the CMOS sensor. On the other hand the measured DQEs for the CCD based system are comparable to laser-based systems with maximum DQEs of 0.54 for $\mathrm{CsBr}: \mathrm{Eu}^{2+}$ and 0.89 for $\mathrm{BaFBr}: \mathrm{Eu}^{2+}$. The BaFBr:Eu ${ }^{2+}$ imaging plate shows a higher DQE than $\mathrm{CsBr}: \mathrm{Eu}^{2+}$ which is attributed to the former absorbing more $59.5 \mathrm{keV}$ X-ray photons. Table 4.2 shows the percentage absorption of both $\mathrm{BaFBr}$ and $\mathrm{CsBr}$ for various energies. At $60 \mathrm{keV}$ (approximately the X-ray energy of interest), BaFBr absorbs $20 \%$ more than CsBr. The differences in DQE for the camera system as opposed to the commercial scanners is attributed to the latter using polychromatic X-ray beams.

\subsection{Images}

Some representative images taken using the $\mathrm{CsBr}: \mathrm{Eu}^{2+}$ commercial imaging plate are shown in figure 4.10 . Figure $4.10(\mathrm{a})$ is a composite image (four images stitched together) of a tarakihi fish taken using the Faxitron X-ray set and CMOS camera. Figure $4.10(\mathrm{~b})$ is an image of a blocked water pump taken with the CMOS camera and Faxitron X-ray set. Figure 4.10(c) is a composite image of a hand taken using the dental X-ray set and CMOS camera. Figures 4.10(d) and 4.10(e) are images of the same finger taken with the CCD and CMOS camera respectively using the dental X-ray set. Medical type images were taken with doses of $0.3-0.4 \mathrm{mSv}$. 


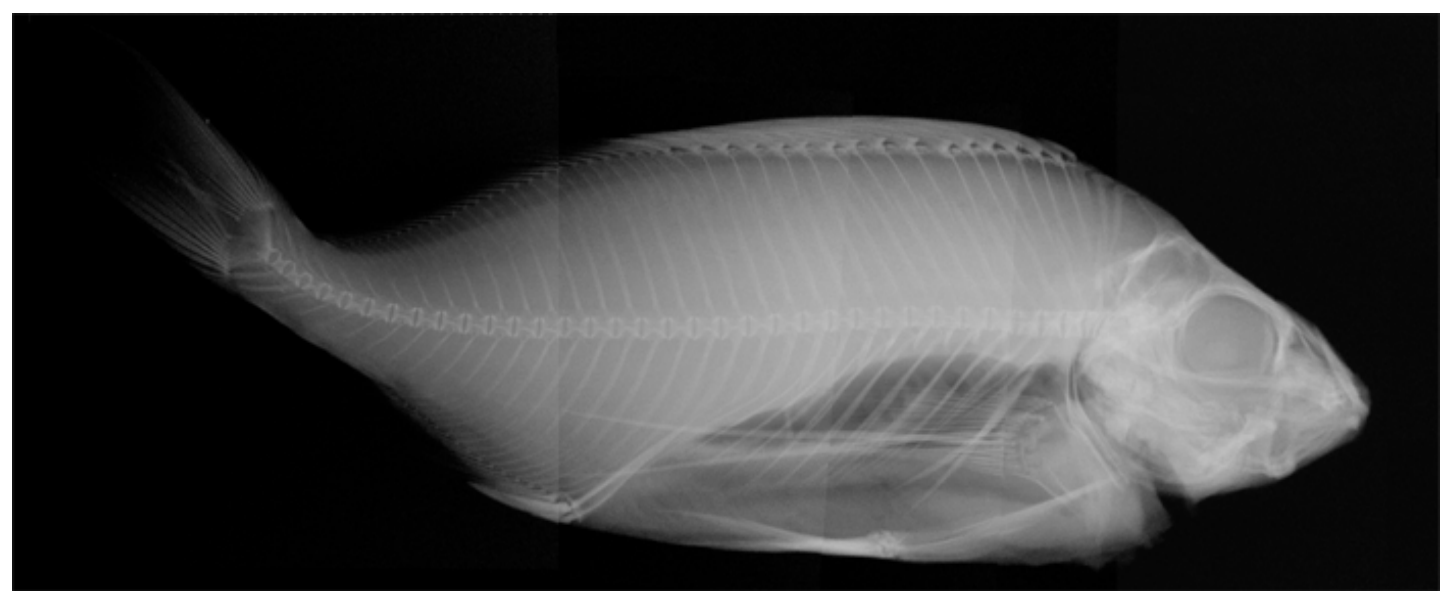

(a)
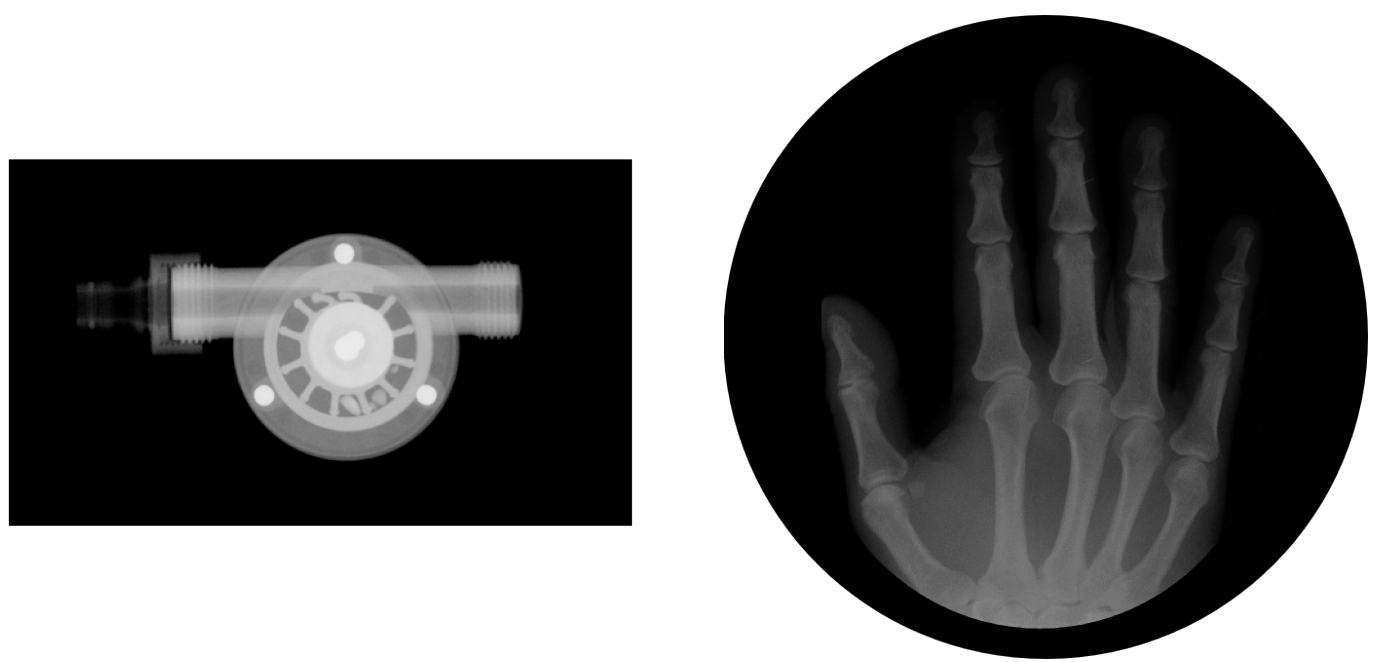

(b)

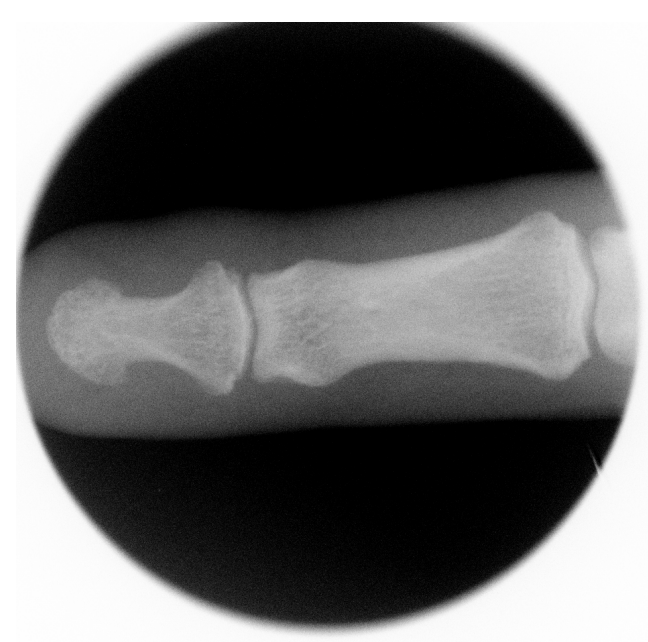

(d)

(c)

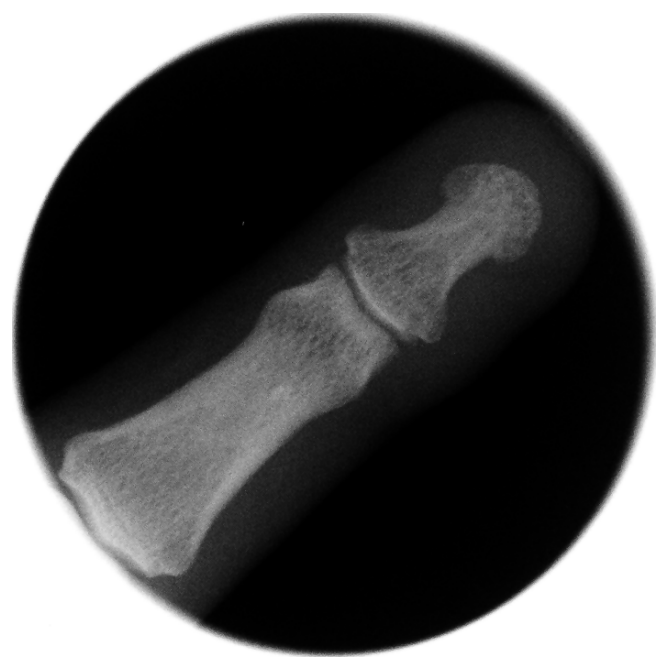

(e)

Figure 4.10: X-ray images taken using the prototype imaging system of a (a) tarakihi fish, (b) water pump, (c) hand, (d) and (e) index finger, as explained in the text. 


\subsection{Discussion}

The digital camera based read-out system described in this chapter is capable of recording X-ray images for both $240 \times 180 \mathrm{~mm}^{2}$ commercial imaging plates and developmental materials. This technique also includes the capacity for high quality photographic images with or without registered X-ray imaging of the same object. The imaging is digital in character, and so post-recording imaging processing is possible. Due to the low power requirements and robust parts a portable imaging system is easily established, and field-based imaging is possible, with the use of a laptop computer and portable X-ray generator. This digital camera based system has many possible applications. It is most suited for non destructive testing or security applications where the dose applied to the object can be high and the resulting signal-to-noise ratio is large. However, images can be obtained with medically acceptable doses, as was shown in figure 4.10, making it suitable for basic level radiology.

The resolution is comparable to that of commercial laser scanning read-out system and is not limited by the camera optics or sensor, but instead by the storage phosphor plate. In terms of the imaging plate, the modest resolution is due to scattering of the PSL light rather than the stimulating light as in the case of the laser based scanning systems. The MTFs show a bimodal type character, especially for the $\mathrm{CsBr}: \mathrm{Eu}^{2+}$ plates, which has been previously observed for glass ceramic storage phosphor materials [56, 57]. The increased bimodal character of the $\mathrm{CsBr}: \mathrm{Eu}^{2+}$ plates is due to their needle-like structure which results in some guiding down the axis of the needle (broad contribution) with some scattering when internal reflection is not satisfied (narrow contribution). The resolution of the CMOS camera based system could be increased by having a black and white sensor which will enable all pixels to be used.

Currently the sensitivity of both systems is limited due to the poor light harvesting of the lens which affects the performance of the system in two ways. 
Firstly, the fraction of PSL reaching the sensor, known as the light collection efficiency (LCE), is quite small. For a Lambertian emitting surface the LCE is given by [58],

$$
\mathrm{LCE}=\frac{T}{\left[1+4 F^{2}(M+1)^{2}\right]},
$$

where $F$ is the f number of the lens, $T$ is the bulk transmission coefficient of the lens, and $M(>1)$ is the demagnification factor between object and image. Estimating the transmission factor to be 0.8 gives an LCE of $0.17 \%$ for this system.

Secondly, there is a limitation on the cassette size, as for a given focal length lens the demagnification factor increases proportionally to cassette size and the LCE is reduced for a large imaging plate.

With the CCD sensor the same image is formed but a smaller fraction is intercepted by the sensor, due to the lens being optimised for full frame sensors. This basic limitation is unlikely to be overcome unless a larger and more costly CCD sensor is used. The sensitivity of the CMOS based system could be increased with a sensor which does not have colour dyes added. In this instance all pixels would be effective in gathering the PSL light, resulting in a factor of four improvement in sensitivity or two in resolution.

In comparing the two implementations both have their advantages and disadvantages. The CCD based system has a high detective quantum efficiency, a linear dose response over three orders of magnitude (wide dynamic range), and a comparable spatial resolution to commercial laser-scanning systems which results in good quality images. However, due to the small sensor, the object size is limited to $83 \times 62 \mathrm{~mm}^{2}$ which is only a fraction $(\sim 1 / 10)$ the area of a typical commercial imaging plate $\left(240 \times 180 \mathrm{~mm}^{2}\right)$. On the other hand, the CMOS based system is capable of recording images from full size imaging plates, again with a comparable resolution but has a narrow dynamic range and low DQE, resulting in low contrast, high noise images. 



\section{Chapter 5}

\section{Synthesis of transparent CsBr:Eu ${ }^{2+}$ imaging plates}

This chapter describes the production process to produce transparent or semitransparent $\mathrm{CsBr}: \mathrm{Eu}^{2+}$ storage phosphor imaging plates. First attempts at synthesising these imaging plates by simply hand grinding a mixture of $\mathrm{CsBr}$ and $\mathrm{EuBr}_{2}$ powders and pressing into a pellet, produced both transparent regions and an X-ray storage phosphor effect characteristic of europium doped CsBr. Surprisingly, by a simple grinding and cold pressing process, the $\mathrm{Eu}^{2+}$ ions have penetrated the CsBr lattice, creating the PSL active $\mathrm{CsBr}: \mathrm{Eu}^{2+}$ centres. With further refinements, it may be possible to produce high resolution, transparent or semi-transparent imaging plates with a simple, cost effective preparation process. This is the objective of the work described in this chapter.

Previous work has shown that water plays a critical role in the PSL activation of $\mathrm{CsBr}: \mathrm{Eu}^{2+}$, and is in fact part of the active PSL site. The PSL active site (shown in figure 5.1 consists of an $\mathrm{Eu}^{2+}$ ion substituting for a $\mathrm{Cs}^{+}$ion, with a water molecule and $\mathrm{a} \mathrm{Cs}^{+}$ion vacancy in two of the nearest neighbour positions. Water can be introduced to the imaging plates in different ways. The Agfa group [33, 34, 35, 36] expose needle imaging plates prepared by co-evaporating CsBr and $\mathrm{EuOBr}$ or $\mathrm{EuBr}_{3}$, to room air at an elevated temperature. The Darmstadt 


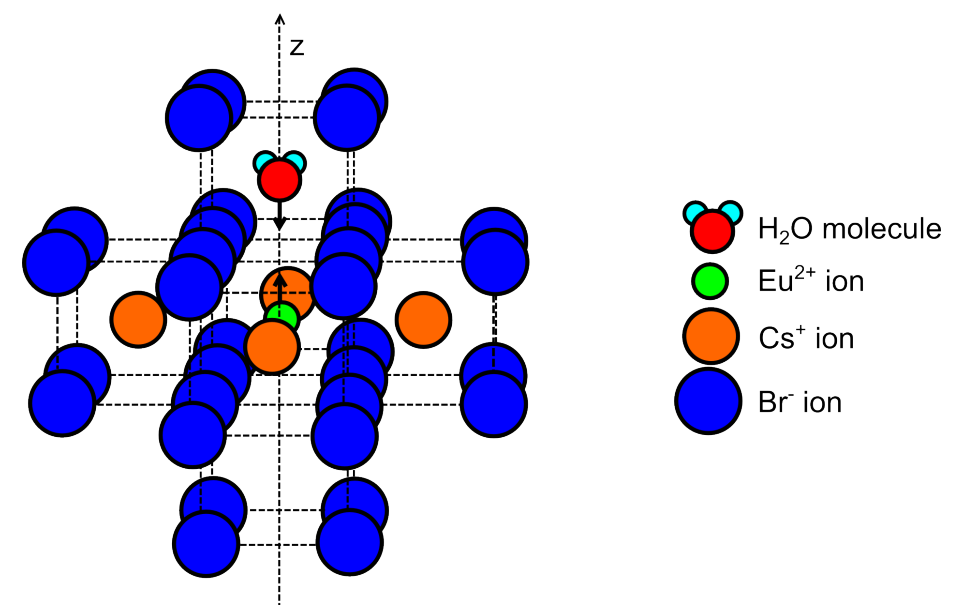

Figure 5.1: PSL active site, as described by Vrielinck et al. [36].

group [29, 30] expose powdered mixtures of $\mathrm{CsBr}$ and $\mathrm{EuBr}_{2}$ to room air, after pre-sintering at a temperature of $600^{\circ} \mathrm{C}$. Therefore, any preparation method will be strongly dependent on humidity and temperature, either during or after the preparation process. Important preparation conditions are, how anhydrous the starting materials are, the humidity during storage and milling of the powders, the temperature during controlled exposure to a humid atmosphere, and the sequence of milling, pressing, and hydration.

Various combinations and sequences of hydration and annealing were attempted in an effort to generate transparent, PSL active $\mathrm{CsBr}: \mathrm{Eu}^{2+}$ pressed imaging plates. The processes investigated were,

1. milling, pressing, followed by hydration in a saturated atmosphere,

2. milling, hydrating in either an ambient or saturated atmosphere, then pressing,

3. milling, pressing, then annealing in vacuum,

4. milling, annealing in an ambient or saturated atmosphere, then pressing, and,

5. sintering, milling, pressing, followed by hydration in a saturated atmosphere. 


\subsection{Preliminary observations}

CsBr:Eu ${ }^{2+}$ imaging plates were first made by batch mixing and grinding 99.8 $\mathrm{mol} \% \mathrm{CsBr}$ and $0.2 \mathrm{~mol} \% \mathrm{EuBr}_{2}$ to a $25 \mu \mathrm{m}$ or less grain size powder. Amounts of approximately $200 \mathrm{mg}$ were weighed and then pressed into pellets in a hydraulic press for two hours under vacuum (approximately 0.1 torr). The forming pressure corresponds to a force of 8 tonnes. The resulting imaging plates have a diameter of $13 \mathrm{~mm}$ and thickness of approximately $300 \mu \mathrm{m}$. The as-made imaging plates show good transparency, as seen in figure 5.2(a), with a transmission of approximately $35 \%$ at the usual stimulation wavelength (633 $\mathrm{nm}, \mathrm{He}-\mathrm{Ne}$ laser) and $11 \%$ at the emission wavelength of $440 \mathrm{~nm}$. Surprisingly, these imaging plates show a storage phosphor effect approximately $30 \%$ that of a commercial needle imaging plate, with no additional treatment. The conversion efficiency (total light output for a given input dose) is $1.5 \mathrm{pJ} \mathrm{mR}^{-1} \mathrm{~mm}^{-3}$ compared to $5.1 \mathrm{pJ} \mathrm{mR}^{-1} \mathrm{~mm}^{-3}$ for a commercial Konica Minolta $\mathrm{CsBr}: \mathrm{Eu}^{2+}$ needle imaging plate. X-ray imaging with the as-made, non-treated imaging plates is possible, as is demonstrated by the X-ray image in figure 5.2(b), which clearly shows the internal circuitry of an integrated circuit (IC) chip (dimensions $8 \times 5$ $\left.\mathrm{mm}^{2}\right)$.

The infrared spectra of the $\mathrm{CsBr}: \mathrm{Eu}^{2+}$ imaging plates gives useful information on the role of water molecules. CsBr itself is transparent to infrared wavelengths,

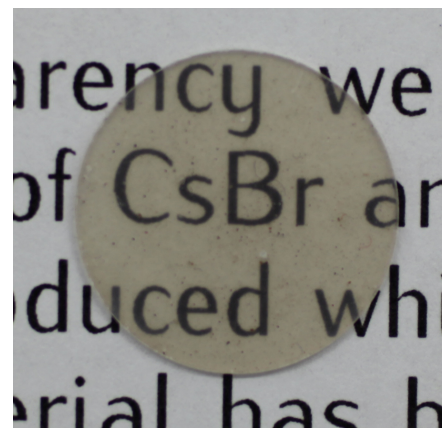

(a)

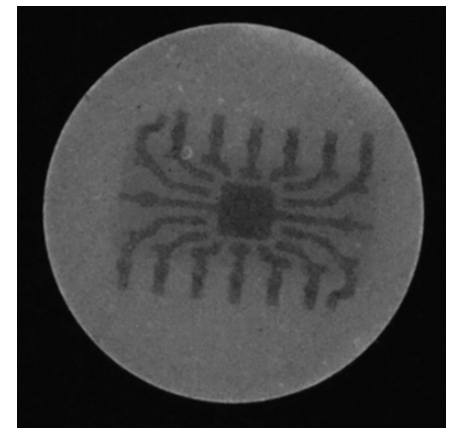

(b)

Figure 5.2: (a) As-made CsBr:Eu ${ }^{2+}$ imaging plate and (b) an X-ray image of an IC chip using an as-made imaging plate. 


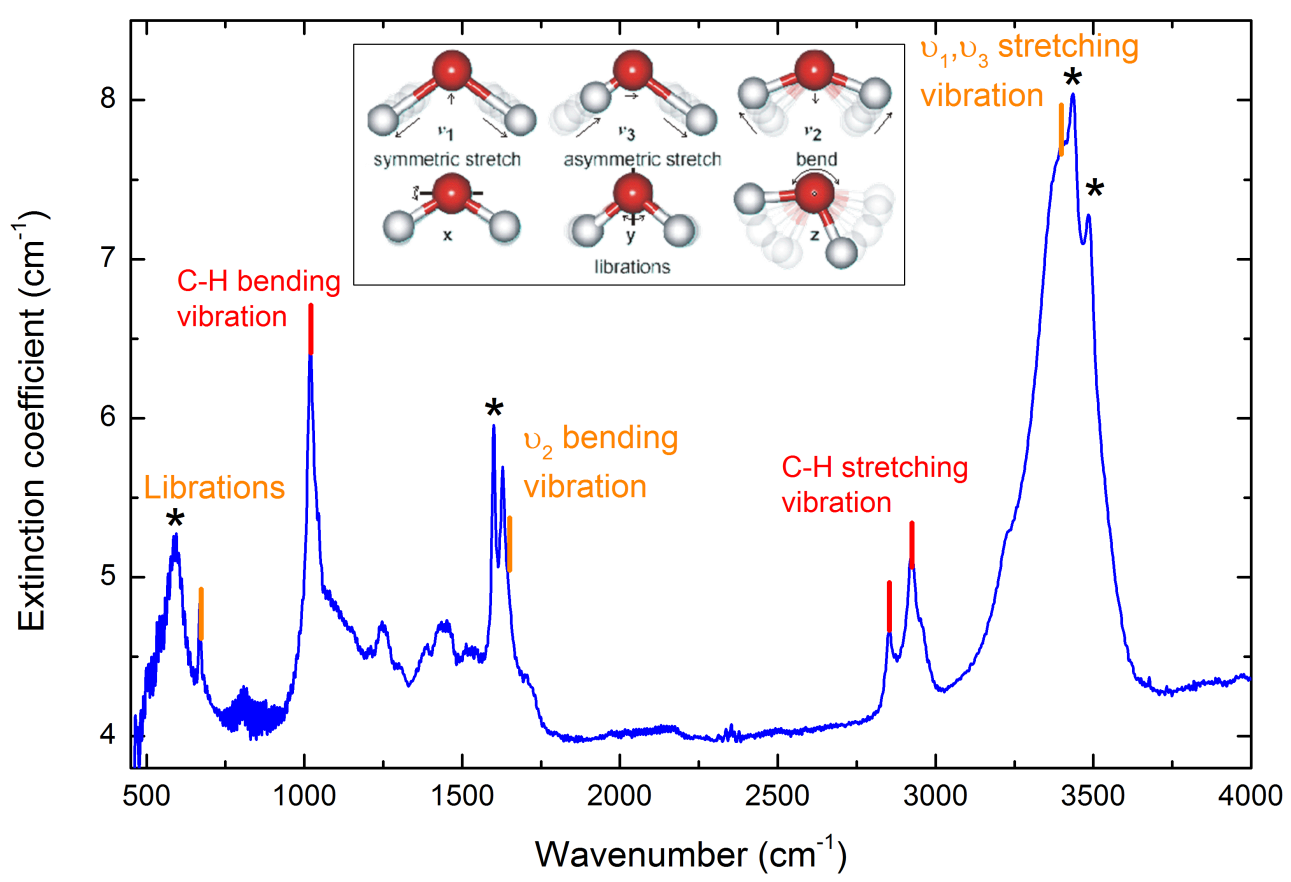

Figure 5.3: Infrared spectrum for as-made $\mathrm{CsBr}: \mathrm{Eu}^{2+}$ imaging plate. The orange markers indicate free liquid water vibrations and librations, the red markers indicate hydrocarbon vibrations and the stars indicate $\mathrm{EuBr}_{2} \cdot 1 \mathrm{H}_{2} \mathrm{O}$ vibrations and librations. Inset: free water molecule vibrational and librational modes [59].

therefore peaks in the IR spectra are most likely associated with free or surface water, any water attached to europium compounds, and possibly hydroxyl ions within the $\mathrm{CsBr}: \mathrm{Eu}^{2+}$ imaging plates. Figure 5.3 shows an example of an infrared spectrum for a milled and pressed $\mathrm{CsBr}: \mathrm{Eu}^{2+}$ imaging plate. The peaks in the spectrum associated with the vibrations of the free liquid water molecule are, the broad band at approximately $3400 \mathrm{~cm}^{-1}$ is the (unresolved) $\nu_{1}$, $\nu_{3}$ symmetric and antisymmetric stretching vibration (refer to inset on figure 5.3), the band at approximately $1600 \mathrm{~cm}^{-1}$ is the $\nu_{2}$ bending vibration and the small band at around $700 \mathrm{~cm}^{-1}$ represents librational modes [59]. The small peaks at around $2900 \mathrm{~cm}^{-1}$ and at approximately $1000 \mathrm{~cm}^{-1}$ [60] are hydrocarbon vibrations thought to be caused by back-streaming of the oil vapour from the rotary pump used during pressing.

The as-made imaging plates (no hydration) have sharp peaks superimposed on the broad unresolved $\nu_{1}, \nu_{3}$ stretching band, an additional peak for the $\nu_{2}$ bending vibration and a separate librations peak at $\sim 600 \mathrm{~cm}^{-1}$ (marked by stars 
Table 5.1: Line positions $\left(\mathrm{cm}^{-1}\right)$ for measured IR spectra (as-made imaging plate) compared to those for $\mathrm{SrBr}_{2} \cdot 1 \mathrm{H}_{2} \mathrm{O}$ [62, 64].

\begin{tabular}{lcccc}
\hline & $\nu_{1}$ stretch & $\nu_{3}$ stretch & $\nu_{2}$ bend & Librational \\
\hline measured & 3485 & 3436 & 1608 & 591 \\
$\mathrm{SrBr}_{2} .1 \mathrm{H}_{2} \mathrm{O}$ & 3488 & 3440 & 1604 & 584 \\
\hline
\end{tabular}

in figure 5.3). The peaks are not due to free or perturbed $\mathrm{OH}^{-}$ions in $\mathrm{CsBr}$ (which has vibrations at 3580 and $3540 \mathrm{~cm}^{-1}$ [61]), and by comparison with non-europium doped $\mathrm{CsBr}$, are associated with the $\mathrm{EuBr}_{2}$ dopant. The $\mathrm{EuBr}_{2}$ must be associated with water molecules for the vibrations to be seen in these locations. One possibility is europium dibromide monohydrate $\left(\mathrm{EuBr}_{2} \cdot 1 \mathrm{H}_{2} \mathrm{O}\right)$. No infrared data for $\mathrm{EuBr}_{2} \cdot 1 \mathrm{H}_{2} \mathrm{O}$ is available, however, IR data is available for strontium bromide monohydrate. $\mathrm{SrBr}_{2} \cdot 1 \mathrm{H}_{2} \mathrm{O}$ is isostructural with the europium compound [62, 63], the ionic radii of $\mathrm{Eu}$ and $\mathrm{Sr}$ are similar (1.18 and $1.17 \AA$ respectively) and the lattice constants are also similar. Table 5.1 shows the comparison of the four principal vibrations of $\mathrm{SrBr}_{2} \cdot 1 \mathrm{H}_{2} \mathrm{O}$ compared to those measured for the as-made imaging plate. The close proximity of the two sets of frequencies suggest the observed peaks are due to $\mathrm{EuBr}_{2} \cdot 1 \mathrm{H}_{2} \mathrm{O}$, and this identification is used in the discussion of the preparation outlined subsequently.

\subsection{Method 1: Milling, pressing and hydrating}

$\mathrm{CsBr}: \mathrm{Eu}^{2+}$ imaging plates were milled, pressed and then hydrated in saturated air $(100 \%$ relative humidity $(\mathrm{RH}))$ at room temperature $\left(\sim 23^{\circ} \mathrm{C}\right)$ for times between 30 seconds and 5 minutes. This atmosphere was achieved by placing a small amount of water at the bottom of a glass desiccator and leaving the desiccator to reach $100 \%$ RH (usually overnight to one day). Imaging plates were hydrated by placing them in the desiccator for the required time and then they were immediately transferred to the dry box to stop the hydration process. Figure 5.4 shows the optical appearance of the imaging plates after the vari- 


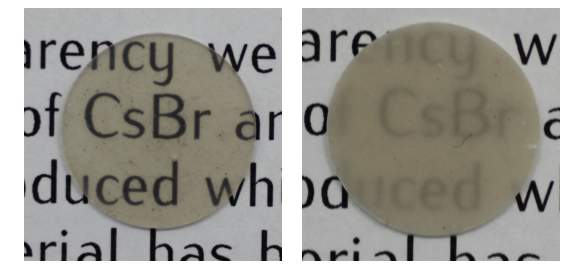

(a) $0 \mathrm{~min}$

(b) $30 \mathrm{~s}$

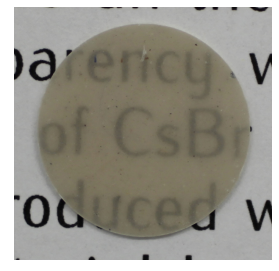

(c) $1 \mathrm{~min}$

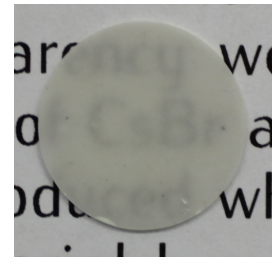

(d) $2 \mathrm{~min}$

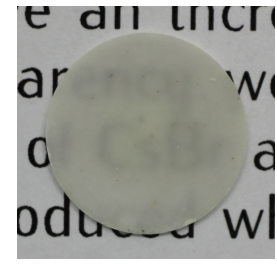

(e) $5 \mathrm{~min}$

Figure 5.4: Optical appearance of $\mathrm{CsBr}: \mathrm{Eu}^{2+}$ imaging plates for various hydration times.

ous hydration treatments. A colour change is seen with increasing hydration time from a brown-tinged transparent imaging plate (no hydration) to a white opaque imaging plate at 5 minutes hydration.

The effects of absorption and scattering within the imaging plates is measured via the extinction coefficient using a Shimadzu UV-Vis 2100 spectrometer, and is given in figure 5.5(a), The extinction spectra for the as-made (not hydrated) imaging plate has two broad bands superimposed on a sloping background. The background is due to scattering in the imaging plates and increases with increasing hydration time. This is the dominant effect causing the decrease in transparency. The two bands in the UV occurring at approximately $270 \mathrm{~nm}$ and $330 \mathrm{~nm}$ correspond to the $4 f^{7} \rightarrow 4 f^{6} 5 d^{1}$ transition of the $\mathrm{Eu}^{2+}$ ion, specifically to the crystal field levels $\mathrm{e}_{\mathrm{g}}$ and $\mathrm{t}_{2 \mathrm{~g}}$ of the $5 d^{1}$ configuration. Any changes in the $e_{g}$ and $t_{2 g}$ band positions with increasing hydration time cannot be seen due to the high scattering background. Figure 5.5(b) shows the transmission coefficient for both $633 \mathrm{~nm}$ light (a commonly used stimulation wavelength) and $440 \mathrm{~nm}\left(\mathrm{Eu}^{2+}\right.$ emission wavelength) for the various hydration times. Initially the transmission is $35 \%$ at $633 \mathrm{~nm}$ and $11 \%$ at $440 \mathrm{~nm}$, which decreases with increasing hydration to $0.1 \%$ for both wavelengths at 5 minutes hydration. To determine the cause of the decrease in transparency (increase in scattering) the structure of the imaging plates were investigated using scanning electron microscopy (SEM). The SEM images of the imaging plates hydrated for various times are shown in figure 5.6. The as-made imaging plates show a pancake-like structure with the plane of the pancakes predominately orientated perpendicu- 


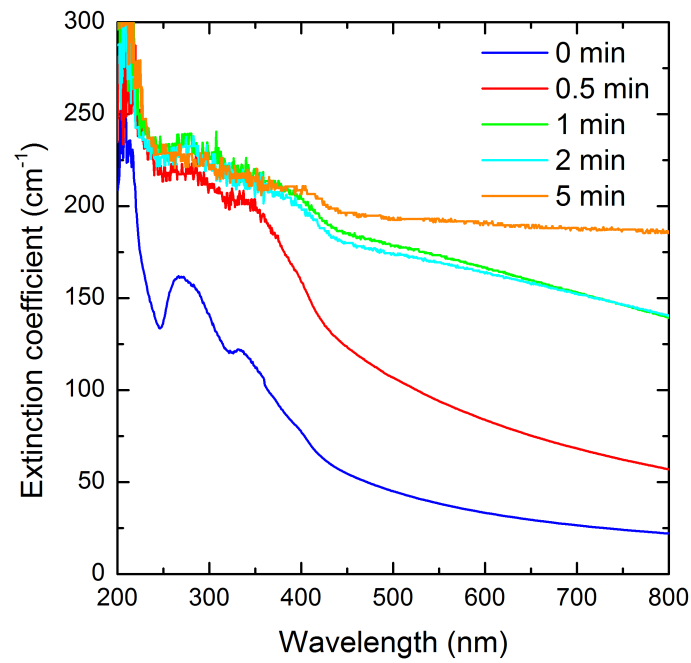

(a)

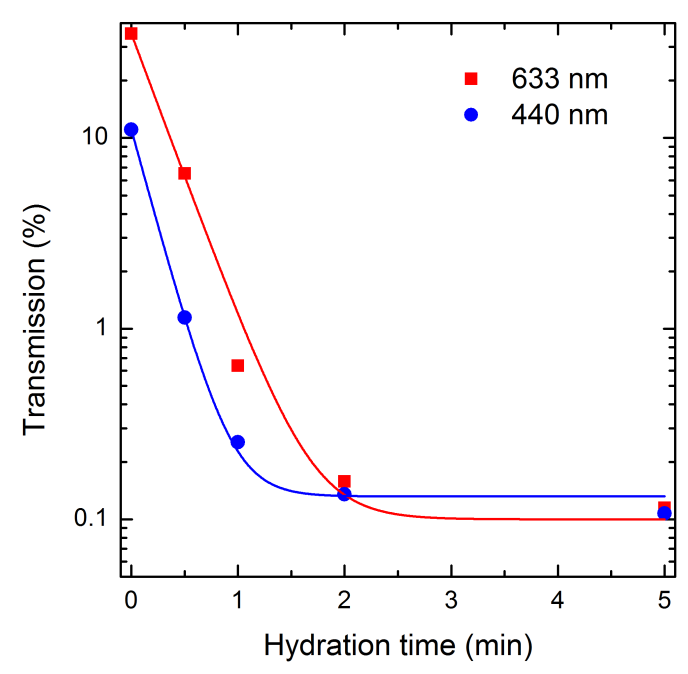

(b)

Figure 5.5: (a) UV-Vis extinction spectra for imaging plates hydrated for various times as shown and (b) transmission coefficient versus hydration time at 633 $\mathrm{nm}$ (red squares) and $440 \mathrm{~nm}$ (blue circles).

lar to the pressing direction. This is caused by plastic compression of the soft $\mathrm{CsBr}$ crystallites in the pressing direction. As the hydration time is increased a recrystallisation of the crystallites occurs initiated from the top and bottom surfaces of the imaging plate. The new crystallites are rounded in shape (figure 5.6(e) and not sharply faceted as might be expected. After the initial formation of the crystallites a thermal roughening process occurs creating the rounded crystallites shown. Arsic et al. [65, 66] have shown that the critical roughening temperature for CsBr crystals in a saturated aqueous solution is $278 \mathrm{~K}$, which is well below the production and hydration temperature used here ( $\sim 296 \mathrm{~K})$. For 30 seconds hydration (figure 5.6(b) the recrystallisation has advanced approximately $40 \mu \mathrm{m}$ into the plate from both top and bottom surfaces. After 1 minute hydration (figure 5.6(c) this recrystallisation depth has increased to approximately $100 \mu \mathrm{m}$. With 2 minutes hydration (figure 5.6(d)) the imaging plate has fully recrystallised with the crystallites having a "diameter" of approximately 1 $\mu \mathrm{m}$. Any further increase in hydration time causes the crystallites to increase in size, as can be seen in figure 5.6(e), Figure 5.6(e) shows (rounded) crystal facets, even though we are above the thermal roughening temperature. This 


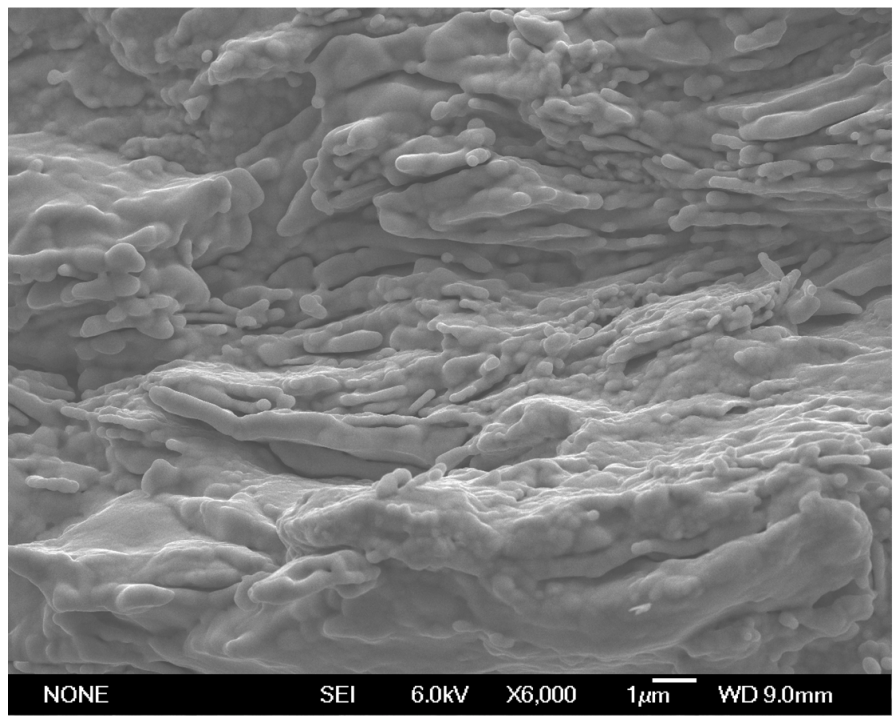

(a) $0 \mathrm{~min}$

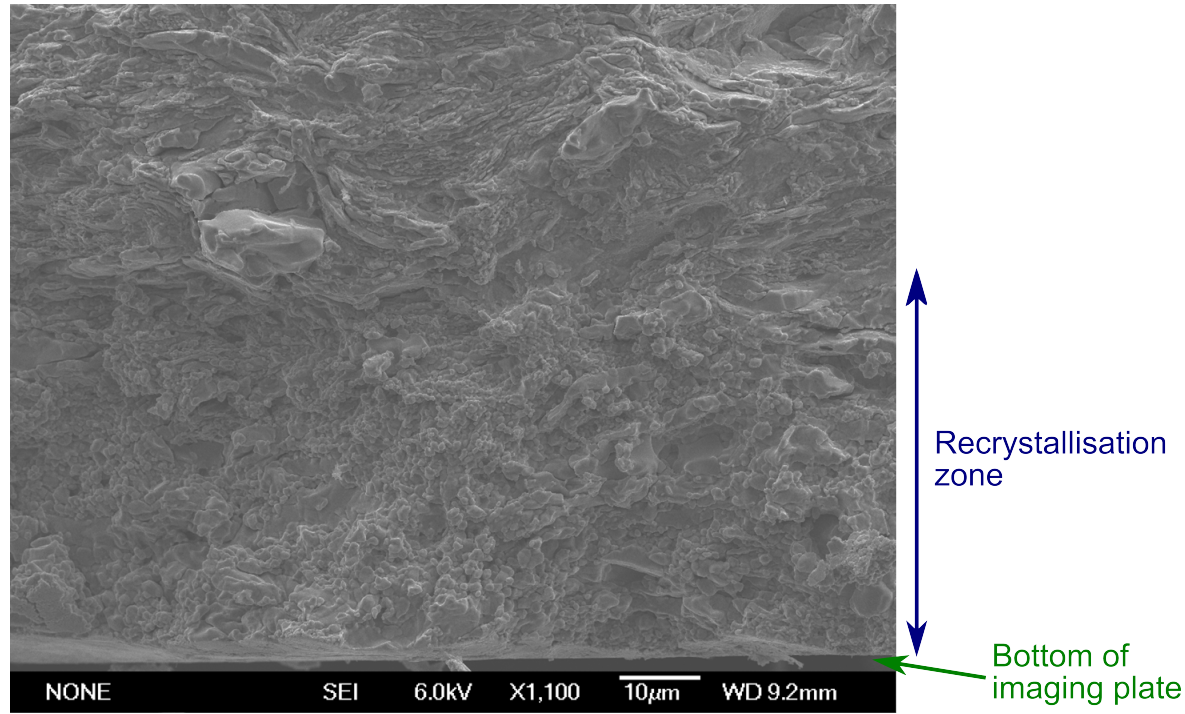

(b) $30 \mathrm{~s}$

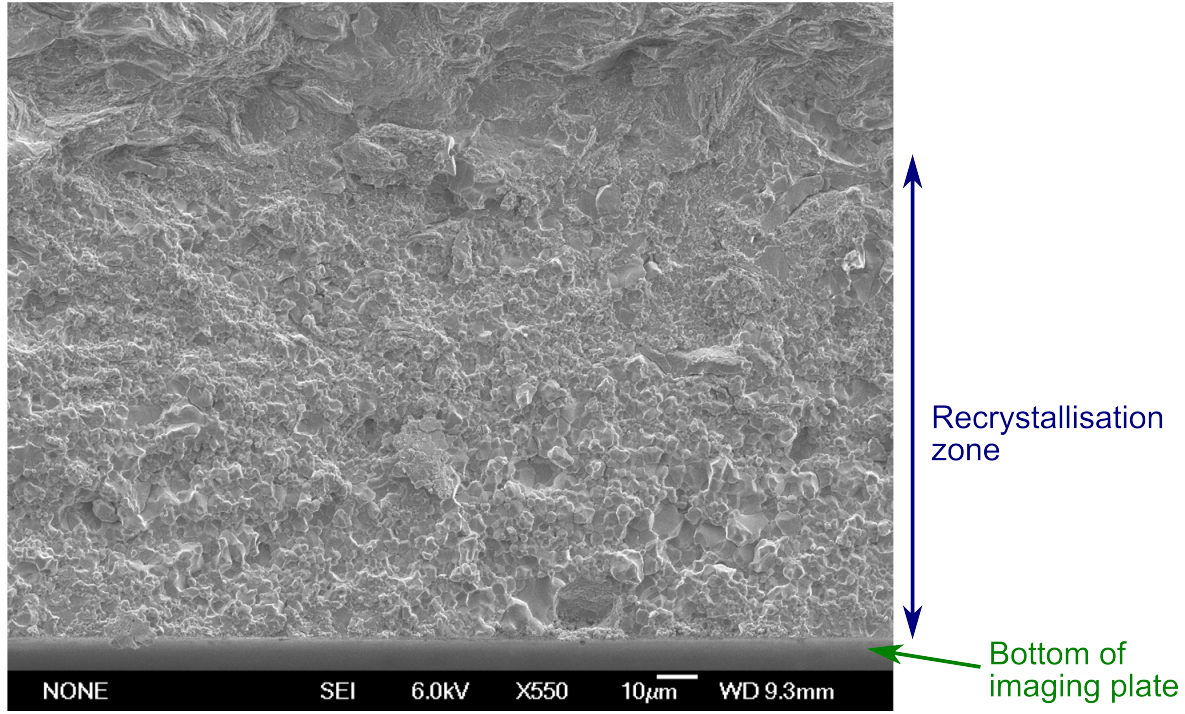

(c) $1 \mathrm{~min}$

Figure 5.6: SEM images, continued on next page. 


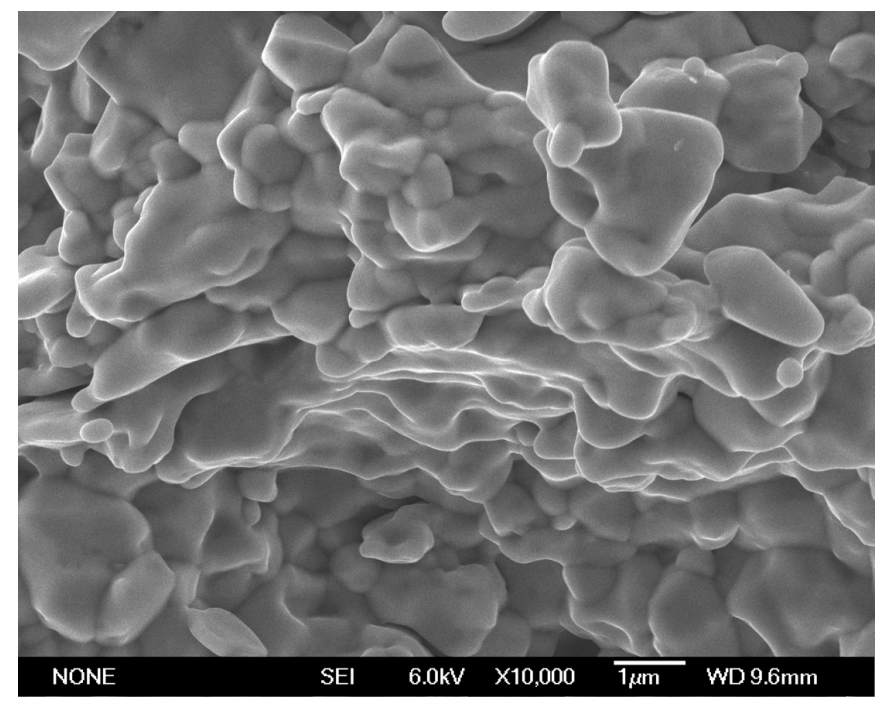

(d) $2 \mathrm{~min}$

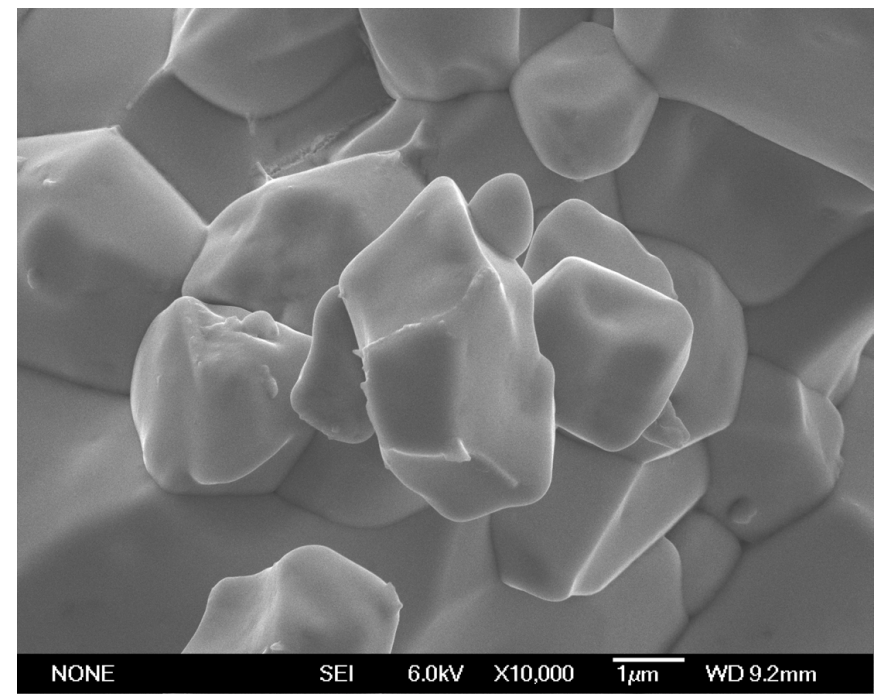

(e) $5 \mathrm{~min}$

Figure 5.6: (cont) SEM images of CsBr:Eu ${ }^{2+}$ imaging plates hydrated for times shown. Note the magnification for images (b) and (c) are reduced to view the effects of recrystallisation.

suggests that the thermal roughening temperature is an average, and there will be both faceted and rounded crystallites at temperatures close to the roughening temperature, depending on the stage of crystal re-growth.

Infrared spectra for the hydrated imaging plates are shown in figure 5.7(a), with an enlarged view of the stretching and bending vibration region in figure 5.7(b). As the hydration time increases the water content of the imaging plate increases and therefore the area under the band increases. Hydrating the imaging plates 


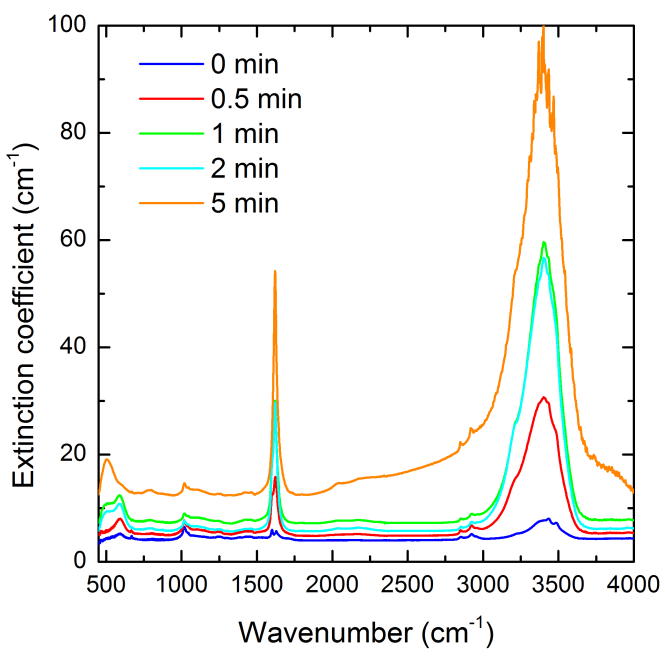

(a)

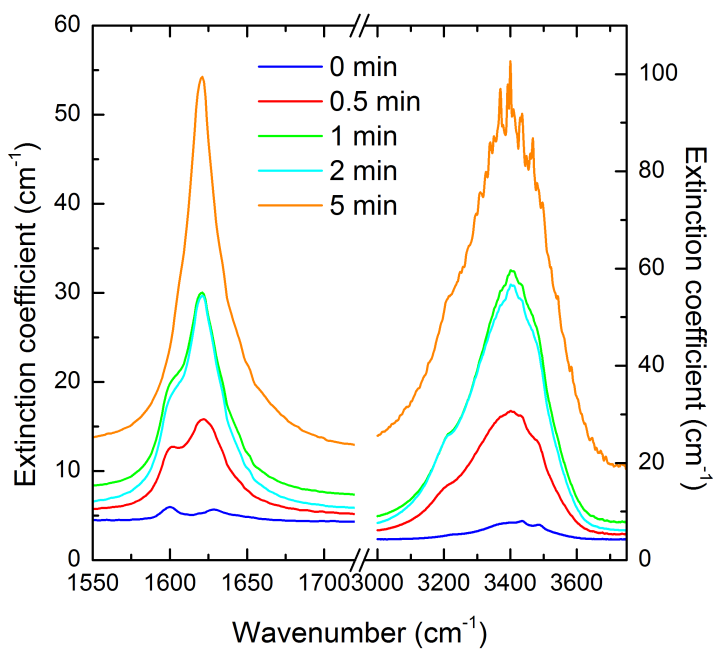

(b)

Figure 5.7: (a) Infrared spectra of $\mathrm{CsBr}: \mathrm{Eu}^{2+}$ imaging plates hydrated for various times. (b) Enlarged view of the $\nu_{2}$ region (left) and $\nu_{1}, \nu_{3}$ region (right).

causes the weak peaks associated with $\mathrm{EuBr}_{2} \cdot 1 \mathrm{H}_{2} \mathrm{O}$ to disappear and the bands due to free water vibrations to increase. For the $\nu_{2}$ bending vibration the peak associated with $\mathrm{EuBr}_{2} \cdot 1 \mathrm{H}_{2} \mathrm{O}$ at $1608 \mathrm{~cm}^{-1}$ decreases with increasing hydration time and the water peak at $1620 \mathrm{~cm}^{-1}$ increases. With 5 minutes hydration the peak associated with $\mathrm{EuBr}_{2} \cdot 1 \mathrm{H}_{2} \mathrm{O}$ has disappeared. The same trend is seen with the $\nu_{1}, \nu_{3}$ vibrations where the peaks at 3485 and $3436 \mathrm{~cm}^{-1}$ gradually disappear with increasing hydration; at 5 minutes the peaks cannot be seen within the noise level. This is assumed to be caused by the $\mathrm{EuBr}_{2}$ dissolving in surface water in the pores. In addition to the disappearance of the $\mathrm{EuBr}_{2} \cdot 1 \mathrm{H}_{2} \mathrm{O}$ vibrations, a new peak at approximately $3200 \mathrm{~cm}^{-1}$ appears. This is correlated with an increasing PSL (figure 5.9) and so it is tentatively assigned to a stretching vibration of the water molecule in the PSL active site identified by Vrielinck et al. [36].

Photoluminescence emission spectra measured at an excitation wavelength of $350 \mathrm{~nm}$ are shown in figure 5.8(a). With no hydration the emission spectrum has three peaks at 420,440 and $485 \mathrm{~nm}$, which are attributed to emission by $\mathrm{EuBr}_{2}$, the PSL active site in $\mathrm{CsBr}$ and an $\mathrm{Eu}^{2+}-\mathrm{Cs}_{\mathrm{v}}$ agglomerate site in CsBr respectively. As the hydration time increases, the 420 and $485 \mathrm{~nm}$ peaks disappear 


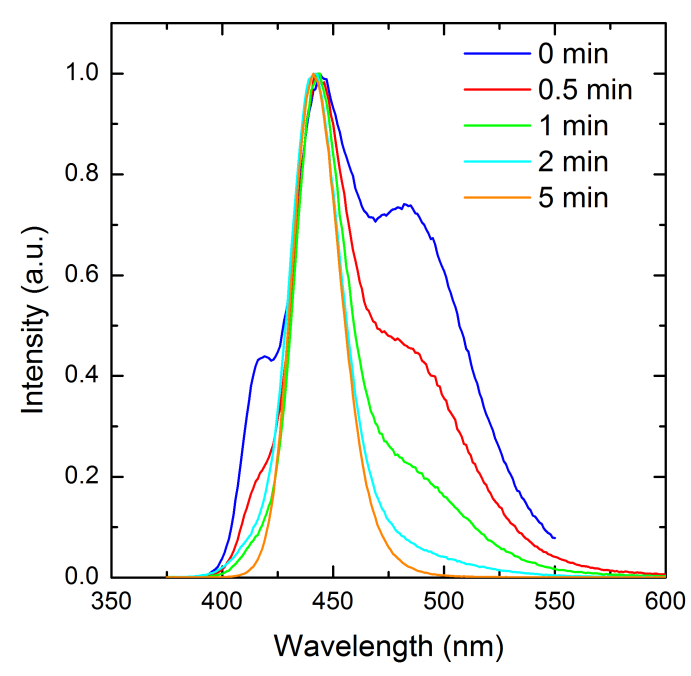

(a)

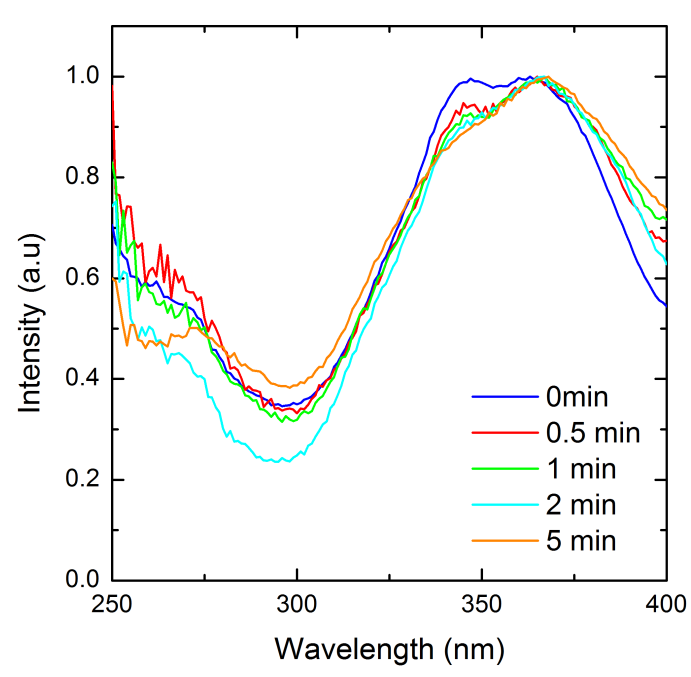

(b)

Figure 5.8: Photoluminescence (a) emission (excited at $350 \mathrm{~nm}$ ) and (b) excitation (440 nm emission) spectra for $\mathrm{CsBr}: \mathrm{Eu}^{2+}$ imaging plates hydrated for various times.

and a single band at $440 \mathrm{~nm}$ remains. The single emission band for the 5 minute hydrated imaging plate is the same as reported for $\mathrm{CsBr}: \mathrm{Eu}^{2+}$ needle imaging plates [11], suggesting the correct PSL active site has been formed. The emission is due to the transition from the $t_{2 g}$ component (split by the axial field) of the $4 f^{6} 5 d^{1}$ configuration to the ground state. The excitation spectra for the $440 \mathrm{~nm}$ emission is shown in figure 5.8(b) and has two main bands at approximately $250 \mathrm{~nm}$ and $360 \mathrm{~nm}$. These correspond with the $e_{g}$ and $t_{2 g}$ states respectively [67]. Splittings of the $t_{2 g}$ state due to the axial crystal field component are unresolved. The small peak at $\sim 340 \mathrm{~nm}$ decreases with increasing hydration time suggesting that it is associated with the $\mathrm{EuBr}_{2}$ or the $\mathrm{Eu}^{2+}-\mathrm{Cs}_{\mathrm{v}}$ agglomerates in the $\mathrm{CsBr}$.

The PSL performance of the imaging plates is measured in terms of the conversion efficiency and stimulation energy, and is shown in figure 5.9. The as-made imaging plate has a stimulation energy of $1.5 \mu \mathrm{J} \mathrm{mm}^{-2}$ and this decreases slightly with increasing hydration time to a value of $1.2 \mu \mathrm{J} \mathrm{mm}^{-2}$. This SE is lower than the $5 \mu \mathrm{J} \mathrm{mm}^{-2}$ measured for $\mathrm{BaFBr}: \mathrm{Eu}^{2+}$ (chapter 3.3) therefore $\mathrm{CsBr}: \mathrm{Eu}^{2+}$ imaging plates require a much lower stimulation energy to read out the PSL, an advantage found previously [25]. No definite value of the stimulation energy 


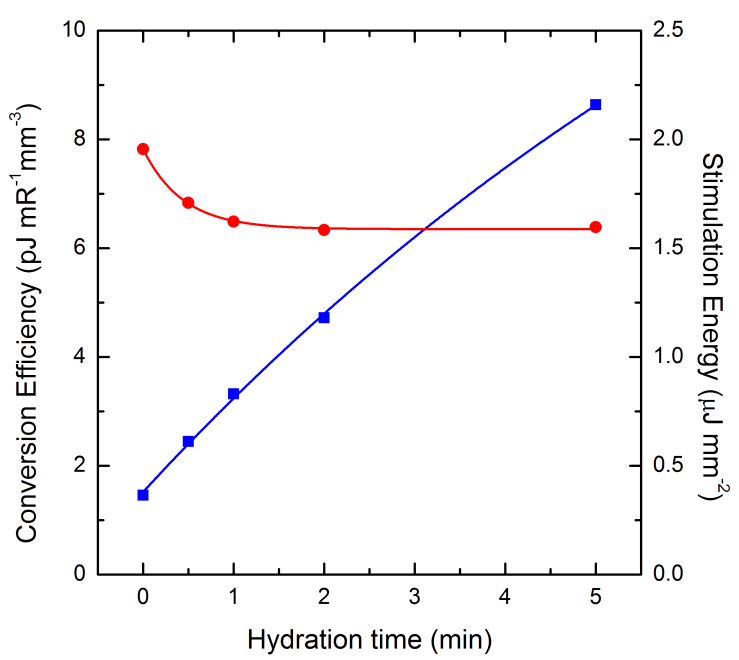

Figure 5.9: Conversion efficiency (blue squares) and stimulation energy (red circles) for $\mathrm{CsBr}: \mathrm{Eu}^{2+}$ imaging plates as a function of hydration time. The lines are a guide to the eye.

for $\mathrm{CsBr}: \mathrm{Eu}^{2+}$ needle imaging plates could be found in the literature. The conversion efficiency is initially $1.5 \mathrm{pJ} \mathrm{mR}^{-1} \mathrm{~mm}^{-3}$ for the as-made imaging plates. Hydrating the imaging plates increases the $\mathrm{CE}$ up to $8.6 \mathrm{pJ} \mathrm{mR}^{-1} \mathrm{~mm}^{-3}$ for the 5 minute hydrated imaging plate. This CE is 1.7 times higher than the 5.1 pJ mR $\mathrm{mm}^{-1} \mathrm{~mm}^{-3}$ measured for the commercial CsBr:Eu ${ }^{2+}$ imaging plate.

The resolution of the imaging plates was measured as described in chapter 3.4 for the various imaging plates, and is shown in figure 5.10. These are supplemented with the optical MTF for the camera system and X-ray MTF for the commercial Konica Minolta needle imaging plate. The X-ray MTFs for the hydrated and non-hydrated imaging plate show a strong bimodal character which is assumed to be caused by PSL light scattering within the imaging plate. A discussion on the bimodal character of the MTFs can be found at the end of the chapter (section 5.7.2). The as-made imaging plate (no hydration) has a resolution of approximately $3 \mathrm{lp} / \mathrm{mm}$ at a MTF of 0.2, and the MTF shows a very strong bimodal character. As the hydration time is increased (transparency decreases) the resolution also decreases, as expected for increased scattering. For hydration times between 30 seconds and 2 minutes the resolution is around $1.5-1.6 \mathrm{lp} / \mathrm{mm}$ and the curves show much the same characteristics. For 5 


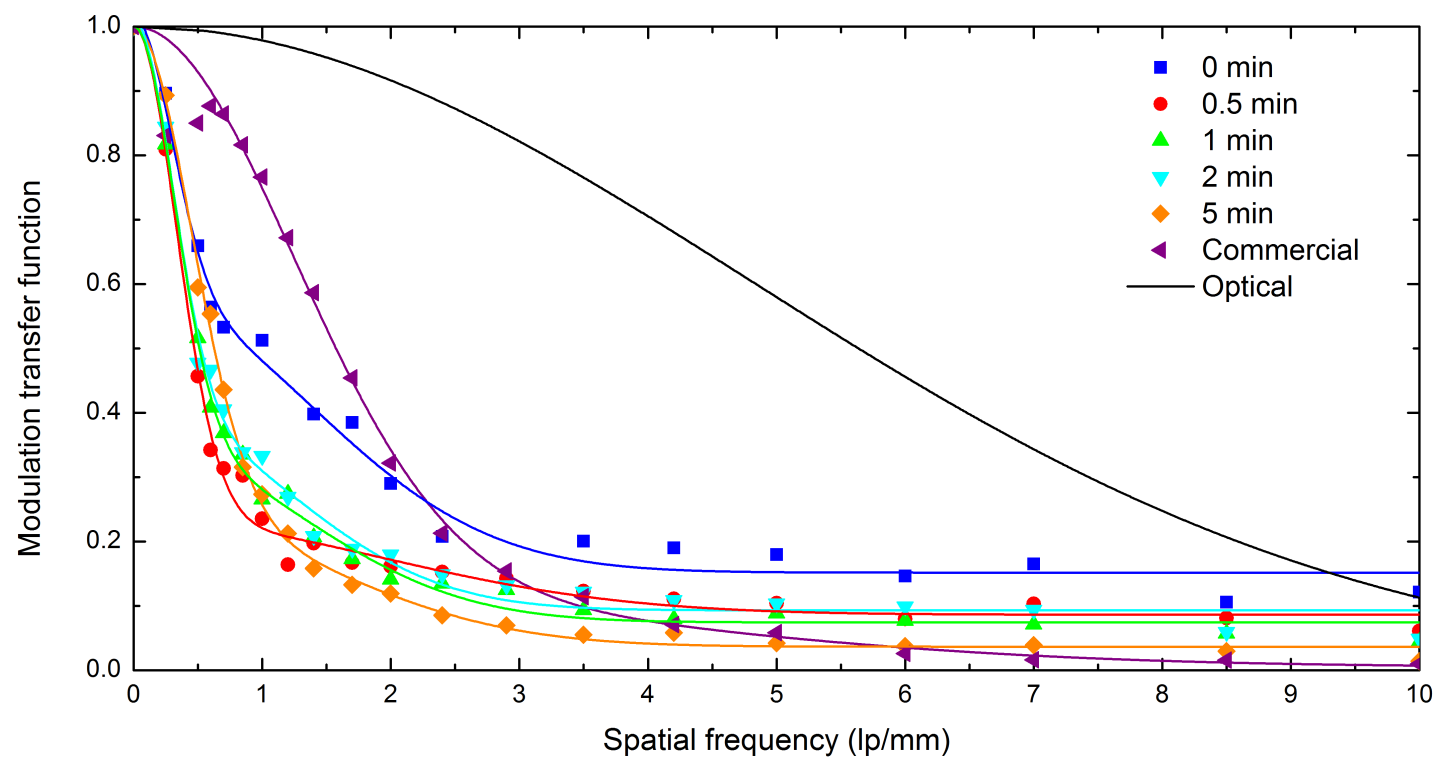

Figure 5.10: Optical and X-ray modulation transfer functions for the camera system and various imaging plates as indicated. The data points have been fitted with the sum of two Gaussians.

minutes hydration the resolution has decreased to $1.2 \mathrm{lp} / \mathrm{mm}$, and the curve has a single Gaussian shape which is attributed to the high opacity and strong scattering of the imaging plate. Only the non-hydrated imaging plate shows a resolution greater than that of the commercial $\mathrm{CsBr}: \mathrm{Eu}^{2+}$ imaging plate. The commercial plate has a resolution of $2.6 \mathrm{lp} / \mathrm{mm}$, and has higher MTF values at low spatial frequencies but lower values at high spatial frequencies. The optical MTF was measured as described in chapter 4.4 and is much greater than any of the X-ray MTFs, showing that the resolution is limited by the imaging plate, not the camera optics.

X-ray images of the high spatial frequency line pairs on the lead grid for the best milled, pressed and hydrated imaging plate and the commercial CsBr:Eu ${ }^{2+}$ imaging plate are shown in figure 5.11. It can clearly be seen that the pressed imaging plates resolve the higher spatial frequency line pairs better than the commercial needle imaging plate. 


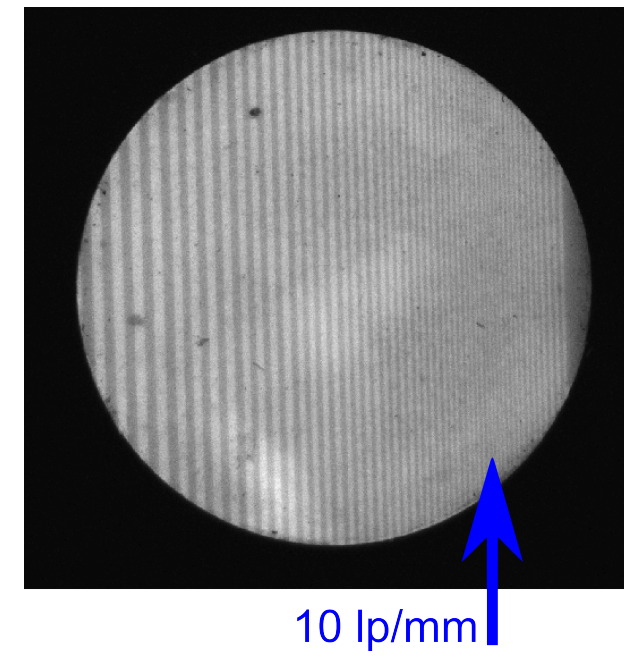

(a)

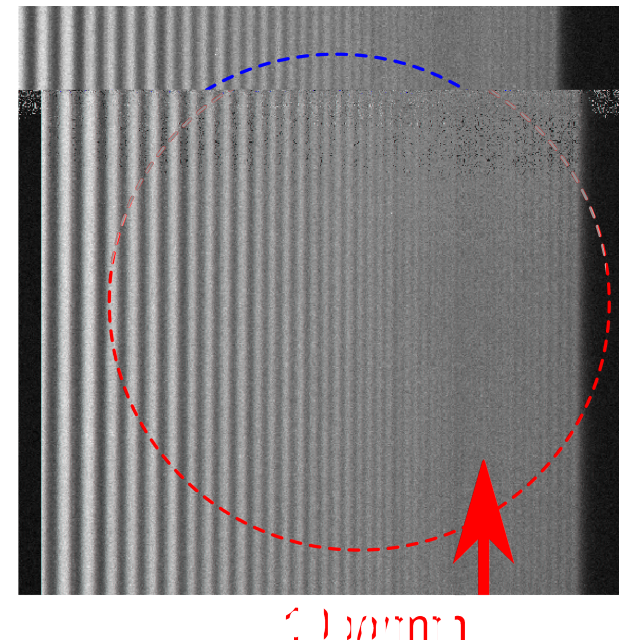

(b)

Figure 5.11: Images of high spatial frequencies of the lead test grid for (a) asmade pressed imaging plate and (b) commercial needle imaging plate. The dashed circle in (b) represents the same spatial frequency range as in (a).

\subsection{Method 2: Milling, hydrating and pressing}

The hydration induced scattering, seen in the previous section, is attributed to the combined effects of free water in the ceramic pores, and to crystal regrowth reducing the contact area between the crystallites. To try and improve the transparency of the imaging plates but still have a high PSL, the milled CsBr and $\mathrm{EuBr}_{2}$ mixture was first hydrated and then pressed into imaging plates. This process was expected to minimise crystallite regrowth and trapped water since the press was operated under vacuum. Imaging plates were made by hydrating powders both in room air $\left(40 \% \mathrm{RH}, 23{ }^{\circ} \mathrm{C}\right)$ and saturated air $(100 \%$ $\left.\mathrm{RH}, 23^{\circ} \mathrm{C}\right)$.

\subsubsection{Room air hydration}

When manufactured in this fashion the imaging plates are inhomogeneous and have both transparent and cloudy areas, as can be seen in figure 5.12. In general, as the hydration time is increased the clouded areas become larger, and the transparent areas become fewer and smaller in size. 


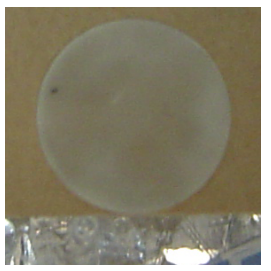

(a) $30 \mathrm{~s}$

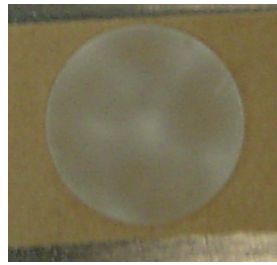

(b) $1 \mathrm{~min}$

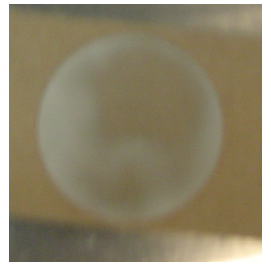

(c) $2 \mathrm{~min}$

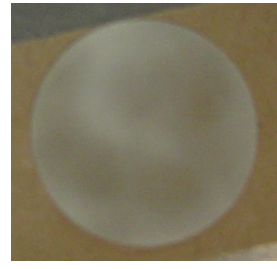

(d) $5 \mathrm{~min}$

Figure 5.12: Images of $\mathrm{CsBr}: \mathrm{Eu}^{2+}$ imaging plates prepared by hydrating as a powder in room air for times shown.

The effect of this on the transparency can be seen in the UV-Vis extinction spectra, and transmission coefficient versus hydration time graph shown in figure 5.13. The UV-Vis extinction spectra show very high noise levels for wavelengths below $350 \mathrm{~nm}$ caused by strong scattering, obscuring the $\mathrm{e}_{\mathrm{g}}$ and $t_{2 \mathrm{~g}}$ peaks. The trend of the transmission is that it decreases with increasing hydration time, apart from a slight increase for 2 minutes hydration. This increase in transmission is due to the large transparent area near the middle of the imaging plate seen in figure 5.12(c). The effect of this is to make the transmission seem larger than it would be if the whole plate was measured (the spectrometer light beam predominantly goes through the middle of the imaging plate). The scatter in figure $5.13(\mathrm{~b})$ is attributed to the optical inhomogeneity in

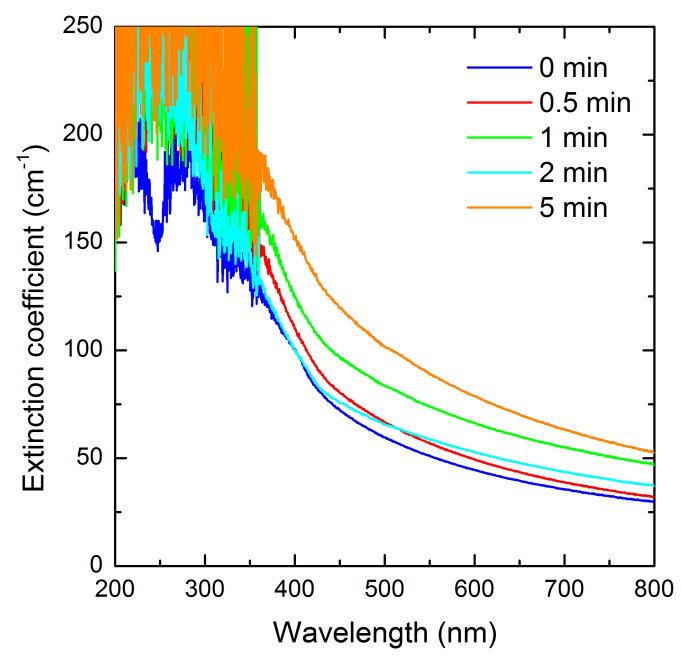

(a)

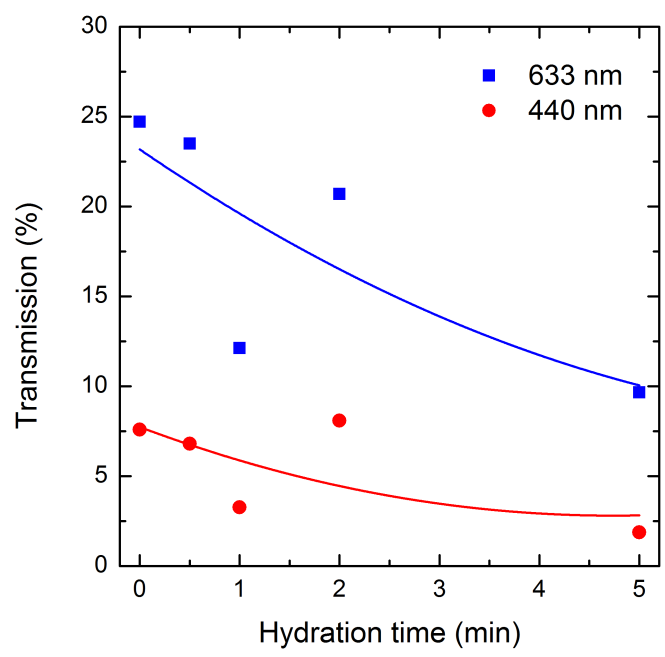

(b)

Figure 5.13: (a) UV-Vis extinction spectra for imaging plates prepared by hydrating as a powder in room air for the times shown and (b) transmission coefficient versus hydration time at 633 and $440 \mathrm{~nm}$. 


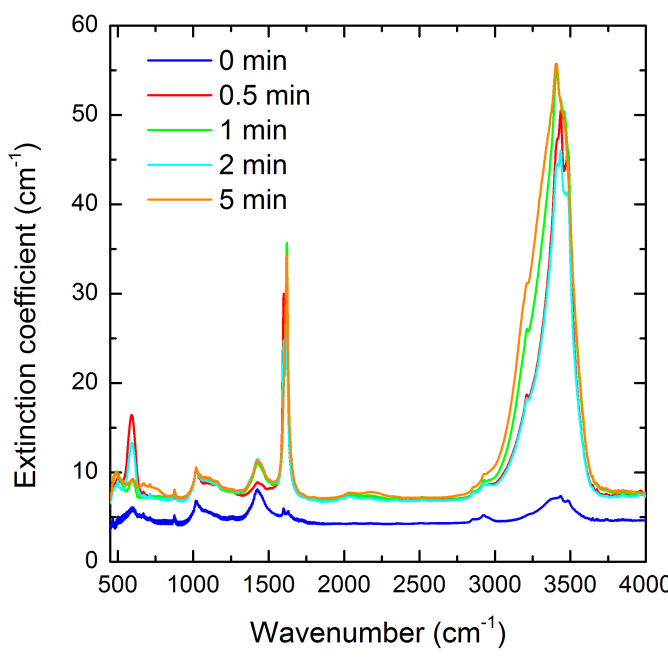

(a)

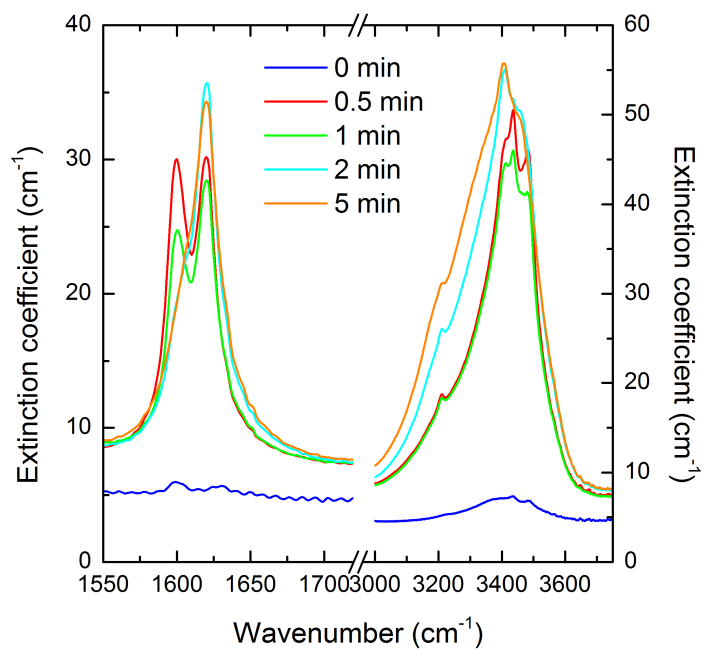

(b)

Figure 5.14: Infrared spectra for imaging plates hydrated in room air as a powder for times shown. (b) Enlarged $\nu_{1}, \nu_{3}$ (right) and $\nu_{2}$ (left) water vibrations.

the imaging plates.

The infrared spectra for the imaging plates hydrated as a powder in room air are shown in figure 5.14. The main bands in figure 5.14 were previously attributed to the free water molecule vibrations. Hydrating the powder for 30 seconds increases the intensity of these bands significantly as compared to the Method $130 \mathrm{~s}$ hydration in figure 5.7. This increase is probably caused by the powder grains having a larger free surface area than a pressed pellet and so more water can be absorbed. It is interesting to note that the uptake of water essentially saturates after just 30 seconds. Figure 5.14(b) shows the changes in the region of the $\nu_{1}, \nu_{3}$ stretching vibration and the $\nu_{2}$ bending vibration in more detail. For hydration times up to and including 1 minute, the spectra clearly show the existence of $\mathrm{EuBr}_{2} \cdot 1 \mathrm{H}_{2} \mathrm{O}$. Hydrating for 2 minutes or longer shows this phase has almost disappeared but the $\nu_{1}, \nu_{3}$ peaks are still not as broadened as those seen in figure 5.7(a). A small side band at approximately $3200 \mathrm{~cm}^{-1}$ again appears immediately with hydration, which we associate with the water molecule in the PSL active $\mathrm{Eu}^{2+}$ site. A second sharp peak is visible at $3400 \mathrm{~cm}^{-1}$ and appears to be correlated with a bend mode line at $1625 \mathrm{~cm}^{-1}$.

PL emission spectra for these imaging plates are shown in figure 5.15(a), The 


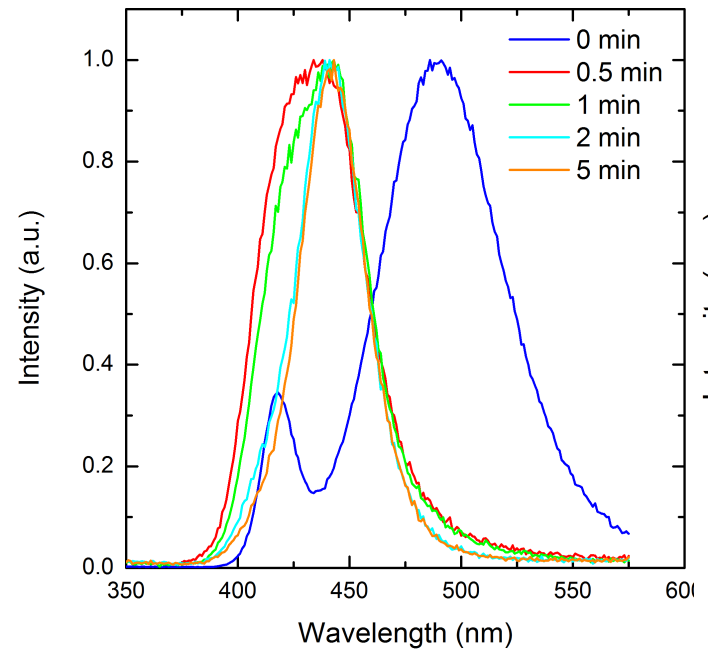

(a)

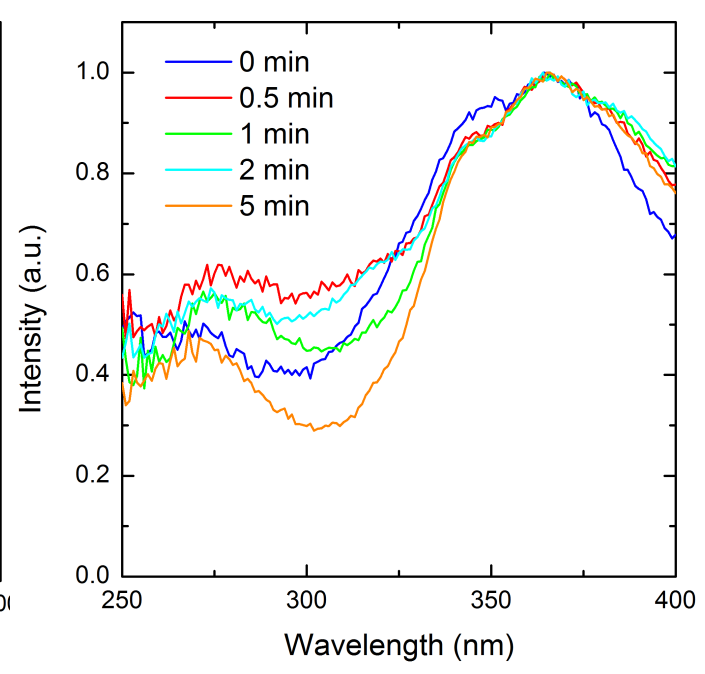

(b)

Figure 5.15: PL (a) emission and (b) excitation spectra for imaging plates hydrated in room air as a powder for the times shown.

as-made imaging plate shows a different spectrum to that found previously (figure 5.8), in that the $485 \mathrm{~nm}$ band is completely dominant, followed by the $420 \mathrm{~nm}$ band and there is no indication of the $440 \mathrm{~nm}$ band. For hydration times up to and including 1 minute the PL spectra shows a mixture of the $\mathrm{EuBr}_{2}$ emission at $420 \mathrm{~nm}$, and the PSL active site at $440 \mathrm{~nm}$. The $420 \mathrm{~nm}$ band is broadened on the low wavelength side which correlates with the appearance of $\mathrm{EuBr}_{2} \cdot 1 \mathrm{H}_{2} \mathrm{O} \nu_{1}, \nu_{3}$ lines in the IR spectra (figure 5.14(b)). Therefore, a band at approximately $415 \mathrm{~nm}$ can be associated with $\mathrm{EuBr}_{2} \cdot 1 \mathrm{H}_{2} \mathrm{O}$. When hydrating the imaging plates for longer than 1 minute only the $440 \mathrm{~nm}$ emission is seen. The PL excitation spectra, shown in figure 5.15(b), show a decrease in the peak at $\sim$ $340 \mathrm{~nm}$, which is associated with a decrease in $\mathrm{EuBr}_{2}$ emission.

The conversion efficiency and stimulation energy for these imaging plates is shown in figure 5.21. As the hydration time increases the CE also increases but only to a maximum of $3.2 \mathrm{pJ} \mathrm{mR}^{-1} \mathrm{~mm}^{-3}$ for 5 minutes hydration. The SE is relatively constant with an increase in hydration time. The scatter in figure 5.16 is attributed to the optical inhomogeneity in the imaging plates. 


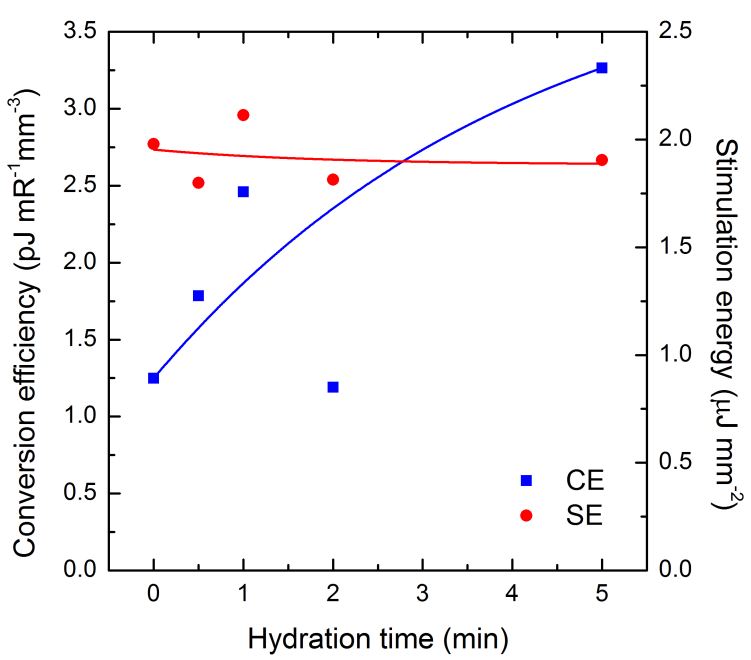

Figure 5.16: CE (blue squares) and SE (red circles) for imaging plates hydrated as a powder in room air as a function of hydration time.

\subsubsection{Saturated air hydration}

The optical appearances of the $\mathrm{CsBr}: \mathrm{Eu}^{2+}$ imaging plates hydrated as a powder in saturated air $(100 \% \mathrm{RH})$ at room temperature are shown in figure 5.17. These imaging plates have a cloudy and inhomogeneous appearance. The 5 minute hydrated imaging plate shows large transparent areas, the reason for which is unclear.

The UV-Vis extinction spectra and transmission coefficient as a function of hydration time for these imaging plates are shown in figure 5.18 . In general the transmission decreases with increasing hydration time. For 5 minutes hydration the transmission increases due to the light beam in the spectrometer predominately passing though the large transparent area seen in the middle of the sample (figure $5.17(\mathrm{c})$ ). The transmission curve shows a much faster decrease

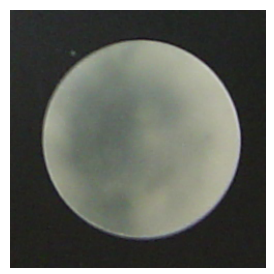

(a) $30 \mathrm{~s}$

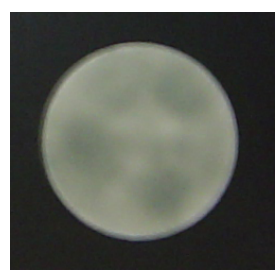

(b) $2 \mathrm{~min}$

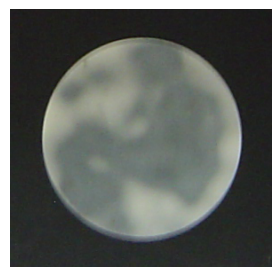

(c) $5 \mathrm{~min}$

Figure 5.17: Images of $\mathrm{CsBr}: \mathrm{Eu}^{2+}$ imaging plates hydrated as a powder in saturated air for times shown. 


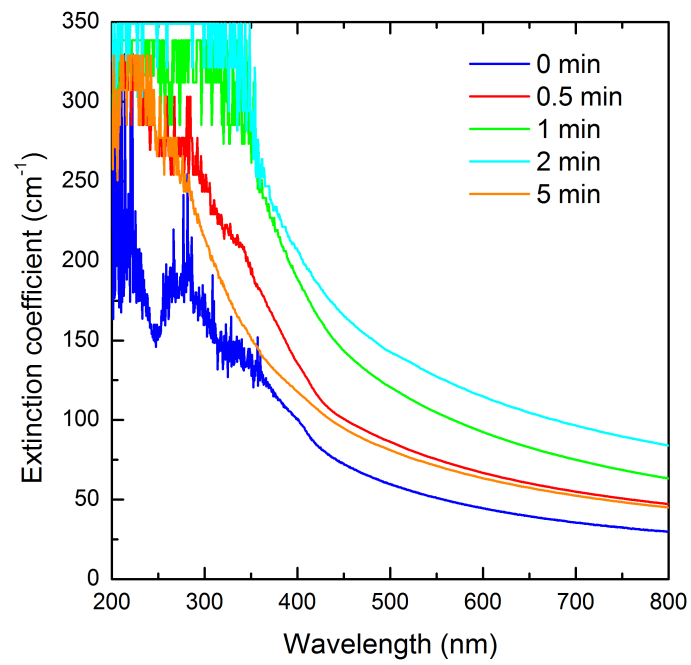

(a)

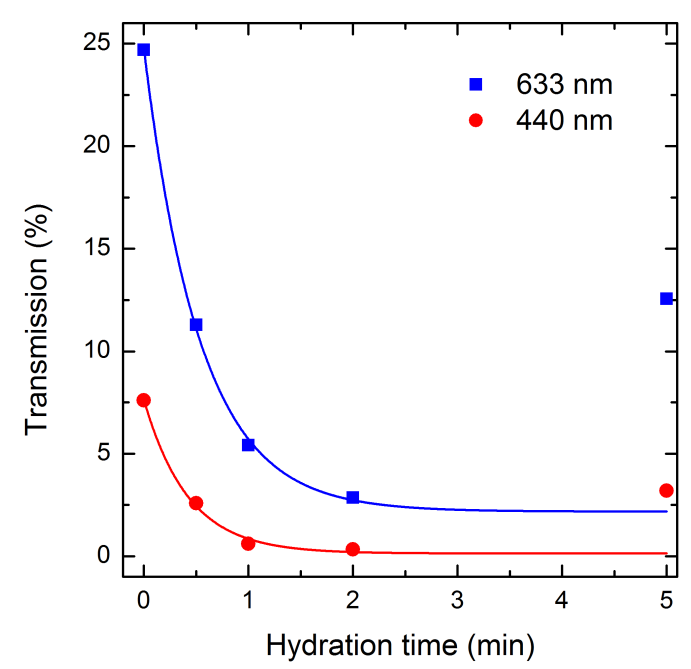

(b)

Figure 5.18: (a) UV-Vis extinction spectra for imaging plate hydrated as a powder in saturated air for the times shown and (b) transmission versus hydration time at 633 (blue squares) and $440 \mathrm{~nm}$ (red circles).

with increasing hydration time as compared to that shown for the imaging plates hydrated in room air (figure 5.13(b)). This is expected as a higher humidity atmosphere contains a greater concentration of water molecules which may be absorbed on the powder, reducing the transparency. The $e_{g}$ and $t_{2 g}$ peaks can just be seen in the noise level of the as-made imaging plate absorption curve, but are not distinguishable for the higher hydrations. However, the onset of the $t_{2 g}$ peak at approximately $400 \mathrm{~nm}$ can be seen to shift to higher wavelengths with increasing hydration time.

The IR spectra for these imaging plates are shown in figure 5.19(a), with an enlarged view of the $\nu_{1}, \nu_{3}$ and $\nu_{2}$ regions in figure 5.19(b), The 30 second hydrated plate shows a similar spectrum to that in the previous section for 5 minute hydration in room air (figure 5.14). When hydrating in saturated air more water molecules are absorbed by the powder and the water content of the imaging plate increases. Hydrating the powder for 1 minute or longer shows the same spectral characteristics as hydrating the whole imaging plate for 30 seconds or longer (figure 5.7). The small peak at approximately $3200 \mathrm{~cm}^{-1}$ seen on the $30 \mathrm{~s}$ hydrated spectrum, and associated with the formation of the 


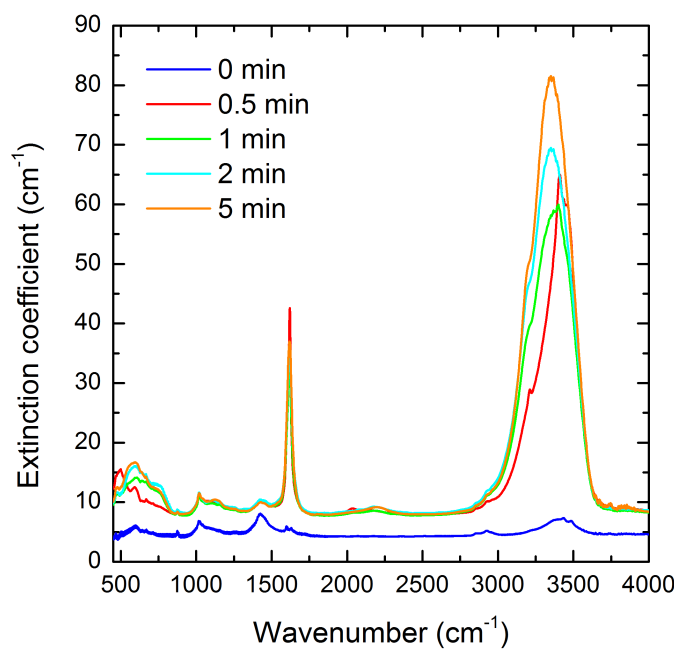

(a)

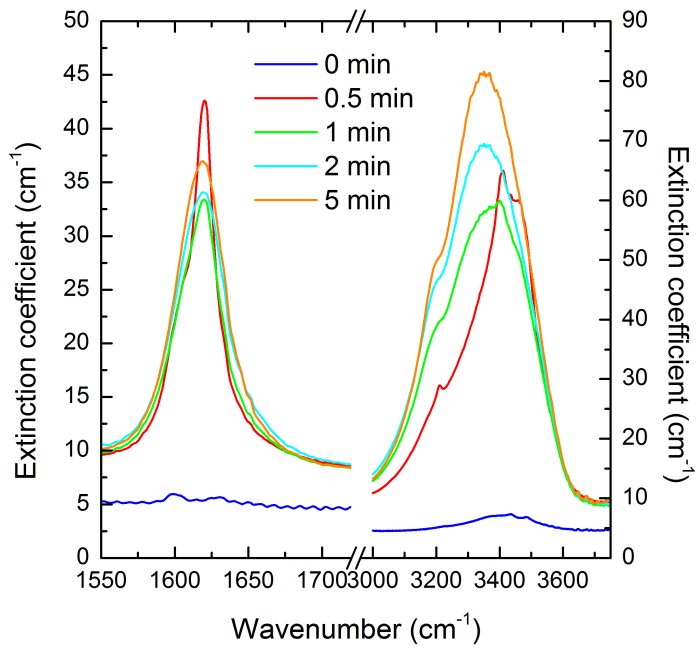

(b)

Figure 5.19: Infrared spectra for imaging plates hydrated in saturated air as a powder for times shown. (b) Enlarged view of the $\nu_{1}, \nu_{3}$ region (right) and the $\nu_{2}$ region (left).

active PSL site, is broadened with a further increase in hydration time. Again a correlation between a stretching peak at $3400 \mathrm{~cm}^{-1}$ and a bending frequency at $1625 \mathrm{~cm}^{-1}$ for short hydration times is seen.

The photoluminescence emission spectra (figure 5.20(a) show an almost immediate change to the PSL active centre emission at $440 \mathrm{~nm}$. For 30 seconds hydration the emission curve has a single band but its peak occurs at a slightly higher wavelength $(443 \mathrm{~nm})$. A further increase in hydration shifts the emission

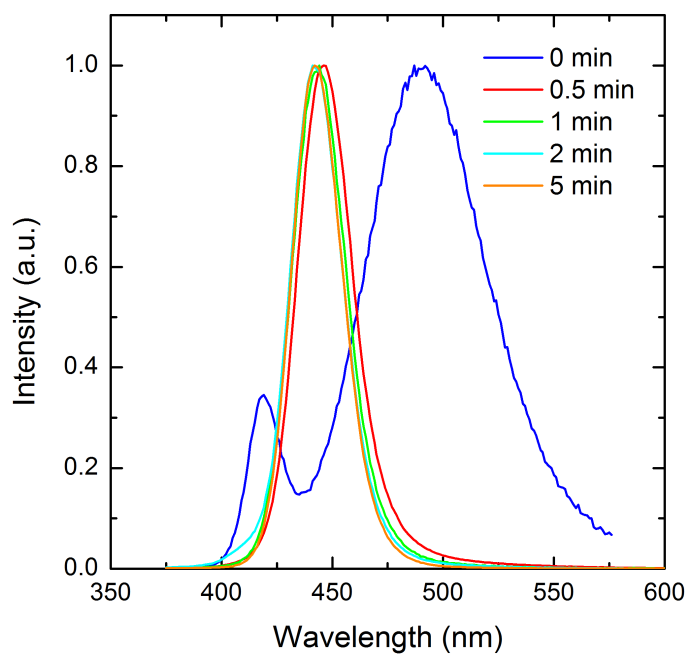

(a)

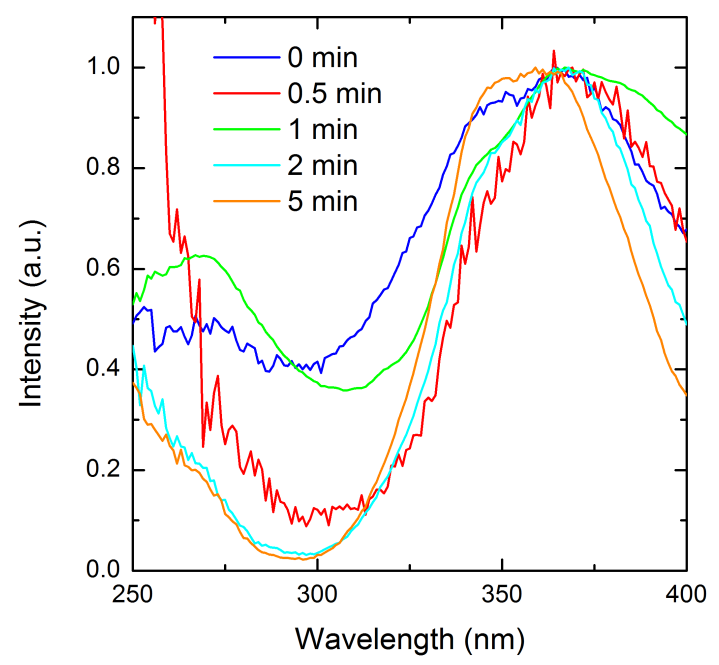

(b)

Figure 5.20: Photoluminescence (a) emission and (b) excitation spectra for imaging plates hydrated as a powder in saturated air for the times shown. 


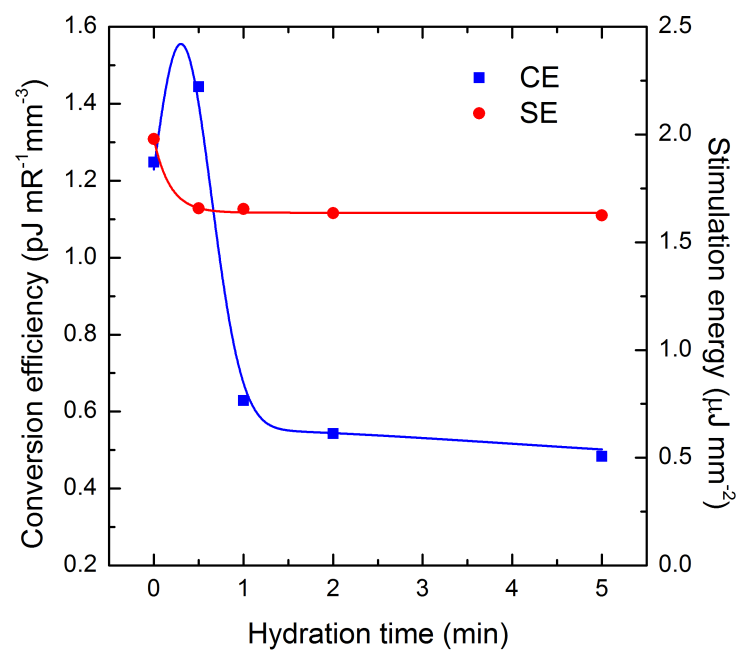

Figure 5.21: CE (blue squares) and SE (red circles) for imaging plates hydrated as a powder in saturated air as a function of hydration time.

to $440 \mathrm{~nm}$. The excitation spectra in figure 5.20(b) show a decrease in the peak at $340 \mathrm{~nm}$, which is associated with $\mathrm{EuBr}_{2}$. The spectrum for the imaging plate hydrated for 5 minutes show a shift to lower wavelengths which is correlated with a decrease in the CE.

The CE and SE for these imaging plates are shown in figure 5.21. The CE is quite low initially and increases to a maximum value of $1.4 \mathrm{pJ} \mathrm{mR}^{-1} \mathrm{~mm}^{-3}$ at 30 seconds hydration. After this the CE decreases to values below the as-made non-hydrated imaging plate. The SE, after an initial decrease from 1.5 to 1.2 $\mu \mathrm{J} \mathrm{mm}^{-2}$ is constant with increasing hydration time.

\subsection{Method 3: Milling, pressing, and annealing}

Imaging plates were prepared as before, but without any (deliberate) hydration. They were then annealed under vacuum (approximately $10^{-5}$ torr) for $15 \mathrm{~min}$ utes at temperatures ranging from $50{ }^{\circ} \mathrm{C}$ to $300{ }^{\circ} \mathrm{C}$ (the un-annealed imaging plate is labelled as $20^{\circ} \mathrm{C}$ ). These imaging plates were produced in the original dry box and therefore have a much higher initial water content; the original dry box had a water content of approximately 25 ppm compared to less than 0.1 ppm for the dry box acquired later in this work. The appearance of the imaging 


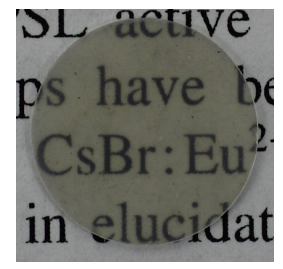

(a) $20^{\circ} \mathrm{C}$

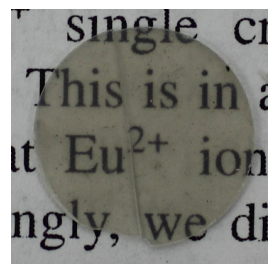

(b) $50{ }^{\circ} \mathrm{C}$

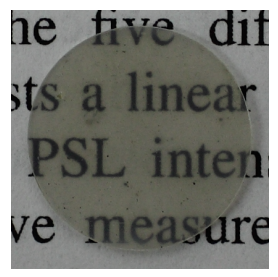

(c) $100{ }^{\circ} \mathrm{C}$

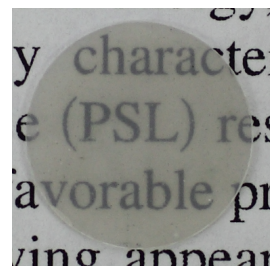

(d) $150{ }^{\circ} \mathrm{C}$

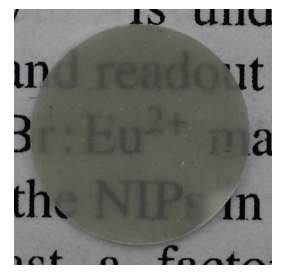

(e) $200{ }^{\circ} \mathrm{C}$

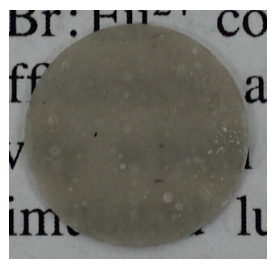

(f) $250{ }^{\circ} \mathrm{C}$

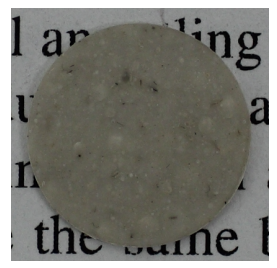

(g) $300{ }^{\circ} \mathrm{C}$

Figure 5.22: Optical appearance of $\mathrm{CsBr}: \mathrm{Eu}^{2+}$ imaging plates annealed at temperatures shown.

plates for the various annealing temperatures is shown in figure 5.22. As the annealing temperature is increased above $100{ }^{\circ} \mathrm{C}$ the transparency decreases and for temperatures greater than $250{ }^{\circ} \mathrm{C}$ the imaging plates are very opaque and show evidence of large scale pores.

These trends are also evident in the UV-Vis extinction spectra and the transmission coefficient at 633 and $440 \mathrm{~nm}$ for the imaging plates shown in figure 5.23. The as-made imaging plate $\left(20^{\circ} \mathrm{C}\right)$ shows the same form of extinction curve as seen previously in figure 5.5(a), but with a slightly higher background level due to the high water content in the original dry box. The initial transmission is $15 \%$ at $633 \mathrm{~nm}$ and $4.5 \%$ at $440 \mathrm{~nm}$. Annealing the imaging plates at temperatures up to $100{ }^{\circ} \mathrm{C}$ causes an increase in the transmission, up to 23 $\%$, which is likely due to some of the surface water being driven off reducing the scattering. The $e_{g}$ and $t_{2 g}$ peaks are seen at approximately 280 and 335 $\mathrm{nm}$ respectively, the latter one as a shoulder. The extinction spectrum of the $100{ }^{\circ} \mathrm{C}$ annealed imaging plate clearly shows the $e_{g}$ and $t_{2 g}$ peaks, with the $e_{g}$ peak shifted to approximately $265 \mathrm{~nm}$, and a change in the relative intensities. Annealing at temperatures above $100{ }^{\circ} \mathrm{C}$ decreases the transmission, and at 300 ${ }^{\circ} \mathrm{C}$ the transmission coefficient is only $\sim 0.03 \%$ at both $633 \mathrm{~nm}$ and $440 \mathrm{~nm}$. The 


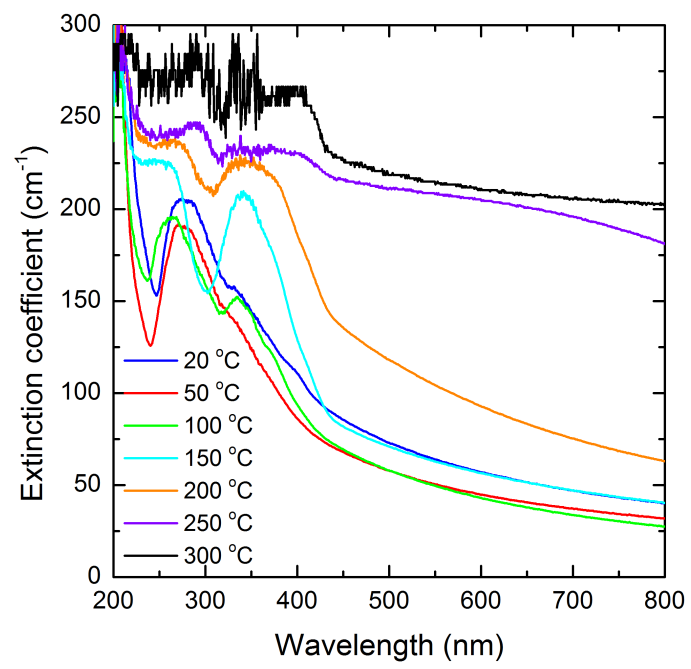

(a)

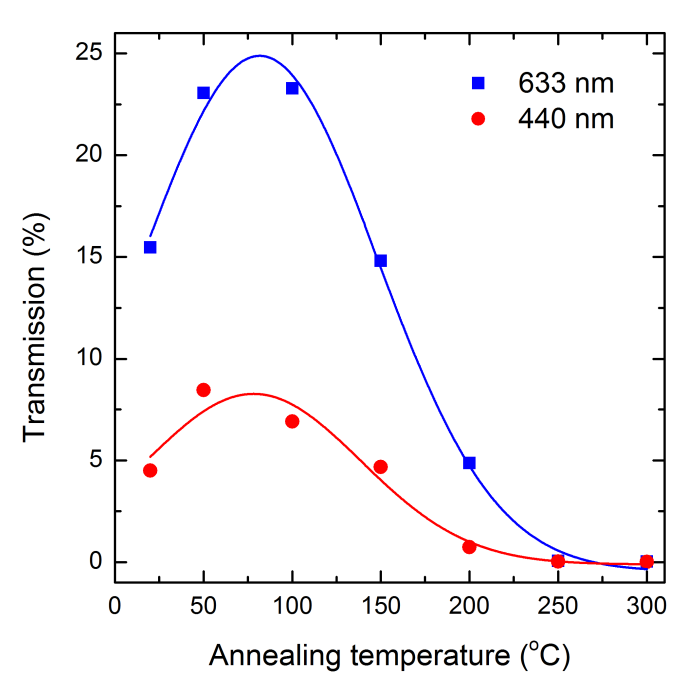

(b)

Figure 5.23: (a) UV-Vis extinction spectra for imaging plates annealed at the temperatures shown and (b) transmission at 633 (blue squares) and $440 \mathrm{~nm}$ (red circles) as a function of annealing temperature.

$e_{g}$ and $t_{2 g}$ peaks seen on the absorption spectra for annealing temperatures of 150 and $200{ }^{\circ} \mathrm{C}$ occur at approximately 265 and $345 \mathrm{~nm}$ respectively. Above $200{ }^{\circ} \mathrm{C}$ the $\mathrm{e}_{\mathrm{g}}$ peak is again shifted to around $290 \mathrm{~nm}$. The shifting of these peaks and their relative intensities suggests a change in the environment of the $\mathrm{Eu}^{2+}$ ion. At low temperatures the $\mathrm{Eu}^{2+}$ mainly occurs as $\mathrm{EuBr}_{2}, \mathrm{EuBr}_{2} \cdot 1 \mathrm{H}_{2} \mathrm{O}$ and as $\mathrm{Eu}^{2+}-\mathrm{Cs}_{\mathrm{v}}$ agglomerates in the $\mathrm{CsBr}$. As the temperature is increased the agglomerates dissociate and the mobile $\mathrm{Eu}^{2+}-\mathrm{Cs}_{\mathrm{v}}$ pairs react with surface water to give the PSL active site. At even higher temperatures the $\mathrm{Eu}^{2+}$ environment changes again, possibly destroying the PSL active site. The processes are further discussed later in this chapter (see section 5.7.1).

The infrared spectra for the imaging plates annealed at various temperatures are shown in figure 5.24. The IR spectra of the plates pre-dated the construction of a dry cell which was used to keep the imaging plates free from air contamination during measurements. Consequently, all the imaging plates apart from the as-made $\left(20^{\circ} \mathrm{C}\right)$ imaging plate have absorbed some additional water vapour during an IR scan. The as-made imaging plate has absorbed less water than the higher temperature annealed imaging plates, which is possibly due to the 


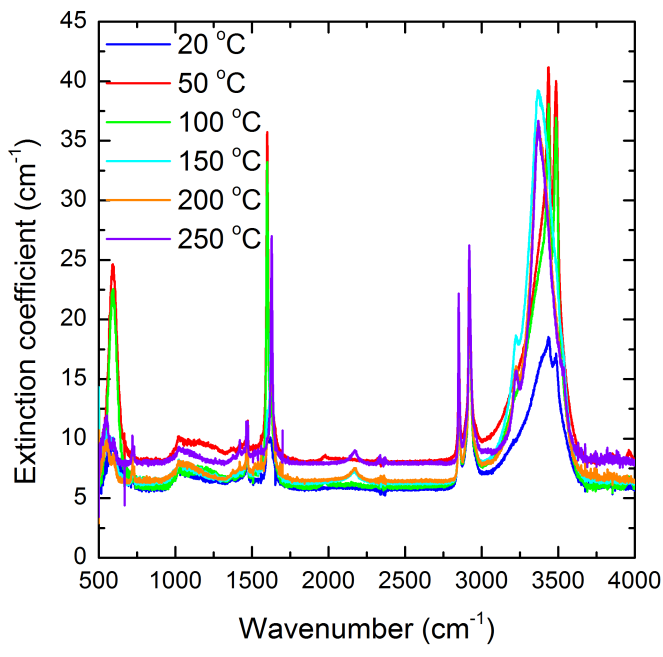

(a)

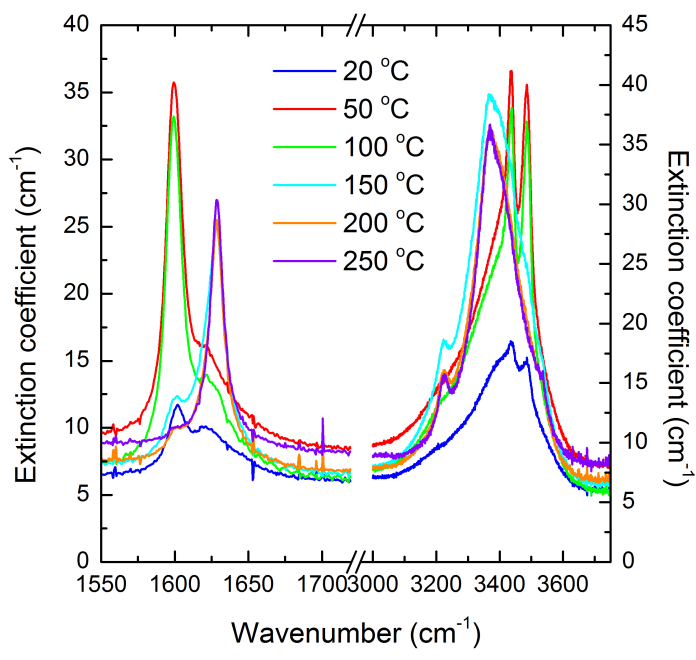

(b)

Figure 5.24: Infrared spectra for $\mathrm{CsBr}: \mathrm{Eu}^{2+}$ imaging plates annealed at the temperatures shown. (b) Enlarged view of the $\nu_{2}$ region (left) and $\nu_{1}, \nu_{3}$ region (right).

heating process making the imaging plate more susceptible to water vapour. The IR curve for $300{ }^{\circ} \mathrm{C}$ has not been included as the high opacity means the peaks cannot be seen above the noise. The two hydrocarbon peaks just below $3000 \mathrm{~cm}^{-1}$ are much larger than seen previously, and in this case are caused by the Nalgene container used for milling the powder (later powder was milled in a stainless steel container to reduce these peaks). Figure 5.24(b) shows an enlarged view of the $\nu_{1}, \nu_{3}$ region and $\nu_{2}$ region. The water content of the as-made imaging plate is much higher than that shown earlier (figure 5.7), due to the higher water content of the original dry box used in this work. Annealing at temperatures up to $100{ }^{\circ} \mathrm{C}$ causes the peaks associated with $\mathrm{EuBr}_{2} \cdot 1 \mathrm{H}_{2} \mathrm{O}$ to increase. This increase is attributed to a process of dissolution of $\mathrm{EuBr}_{2}$ in surface water and recrystallisation as $\mathrm{EuBr}_{2} \cdot 1 \mathrm{H}_{2} \mathrm{O}$ when the imaging plates are cooled back to room temperature. At annealing temperatures greater than 100 ${ }^{\circ} \mathrm{C}$ (above the boiling point of water), the $\mathrm{EuBr}_{2} \cdot 1 \mathrm{H}_{2} \mathrm{O}$ peaks disappear which is attributed to the decomposition of any $\mathrm{EuBr}_{2} \cdot 1 \mathrm{H}_{2} \mathrm{O}$; a TGA measurement shows that the decomposition begins at approximately $140{ }^{\circ} \mathrm{C}$. For temperatures greater than $150{ }^{\circ} \mathrm{C}$, the absence of any $\mathrm{EuBr}_{2} \cdot 1 \mathrm{H}_{2} \mathrm{O}$ IR peaks, and the EPR results presented later, suggests that the europium has reacted with the CsBr. 
The $\nu_{2}$ bending vibration clearly shows the peak at $1608 \mathrm{~cm}^{-1}$ decreasing and the free water $1620 \mathrm{~cm}^{-1}$ peak increasing associated with the disappearance of $\mathrm{EuBr}_{2} \cdot 1 \mathrm{H}_{2} \mathrm{O}$. The side band at $\sim 3200 \mathrm{~cm}^{-1}$, associated with the PSL active site increases in intensity with increasing annealing temperature. Additionally, a peak at approximately $2200 \mathrm{~cm}^{-1}$ is found at annealing temperatures greater than $150{ }^{\circ} \mathrm{C}$, which although not seen previously, is possibly associated with the PSL active site.

For these imaging plates the effect of annealing temperature on PSL was determined using the camera system described in chapter 4 as the integrating sphere apparatus was not operational during the period of this work. The PSL intensity in this case is a relative measurement and only the trends in PSL intensity with annealing temperature can be determined. The PSL intensity (in terms of the camera's blue channel count) for the various annealing temperatures is shown in figure 5.25. The PSL increases with increasing annealing temperature, and reaches a maximum value at around $250{ }^{\circ} \mathrm{C}$. Above $250{ }^{\circ} \mathrm{C}$ the PSL intensity either decreases with a further increase in annealing temperature or remains constant. More measurements at temperatures above $300{ }^{\circ} \mathrm{C}$ would need to be made to determine the trend.

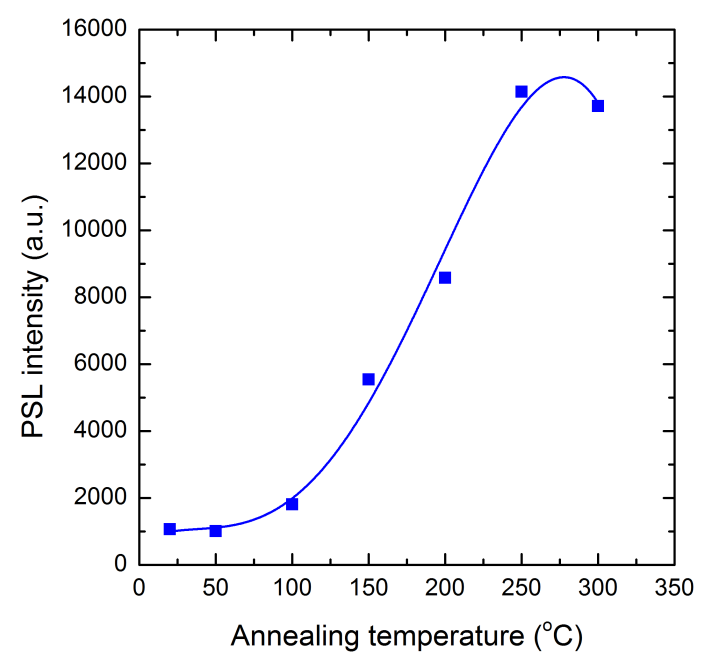

(a)

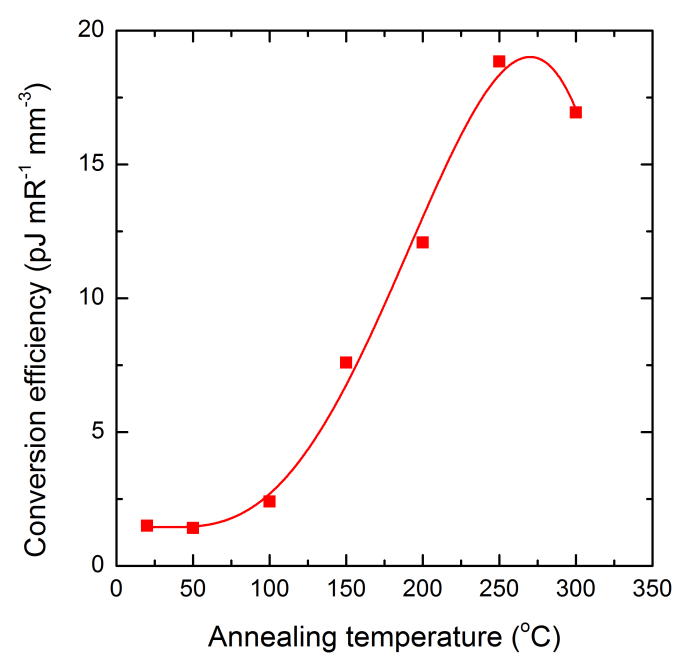

(b)

Figure 5.25: (a) PSL intensity measured using the camera system and (b) calculated $\mathrm{CE}$ for $\mathrm{CsBr}: \mathrm{Eu}^{2+}$ imaging plates as a function of annealing temperature. 
The conversion efficiency for these imaging plates can be estimated by assuming the as-made plate has the same $\mathrm{CE}$ as in section 5.2 (it is likely to be higher due to the higher water content). These calculated conversion efficiencies are shown in figure $5.25(\mathrm{~b})$, The $\mathrm{CE}$ for an imaging plate annealed at $250{ }^{\circ} \mathrm{C}$ would be approximately $18.8 \mathrm{pJ} \mathrm{mR}^{-1} \mathrm{~mm}^{-3}$, which is approximately four times greater than for the commercial imaging plate. Given the earlier comments, this should be a lower estimate.

The EPR spectra for the CsBr:Eu ${ }^{2+}$ imaging plates annealed at temperatures from 50 to $400{ }^{\circ} \mathrm{C}$ are shown in figure 5.26. The extra temperatures of 350 and $400{ }^{\circ} \mathrm{C}$ were added to complete the EPR pattern, although no other measurements were made. Initially the un-annealed imaging plate shows a spectrum which corresponds to that seen for $\mathrm{EuBr}_{2}$. After annealing at $150{ }^{\circ} \mathrm{C}$ the EPR spectrum has changed to that expected for the active PSL $\left(\mathrm{Eu}^{2+}-\mathrm{Cs}_{\mathrm{v}}-\mathrm{Cs}_{\mathrm{v}}\left(\mathrm{H}_{2} \mathrm{O}\right)\right)$ site as described by the Agfa group [33, 34, 35, 36], and shown in figure 5.1] at the start of this chapter. The structure seen in the powder spectrum corresponding to the PSL active site is not simply the seven $\delta m_{s}= \pm 1$ allowed transitions for a $S=\frac{7}{2}$ system. For a single crystal the spectrum seen depends on the relative orientation of the crystal to the applied magnetic field, which can be very different for each orientation. In a powder, the crystal are orientated randomly, and the spectrum seen is a sum over all possible orientations. The features in the spectrum then correspond to so-called turning points where the rate of change of the resonant field strength with orientation is zero; such orientations give a strong contribution to the observed spectrum. Annealing at temperatures above $250{ }^{\circ} \mathrm{C}$ gives another spectrum change, to one which was again seen by Loncke et al. [33] in the case of over-annealing (annealing at temperatures which reduce the PSL intensity). This spectrum, comprising of a single broad line, is characteristic of $\mathrm{Eu}^{2+}$ at a site with only a very small zero-field splitting, possibly an isolated $\mathrm{Eu}^{2+}$ ion at a site of octahedral symmetry.

To confirm the EPR centre observed here for annealing temperatures between 


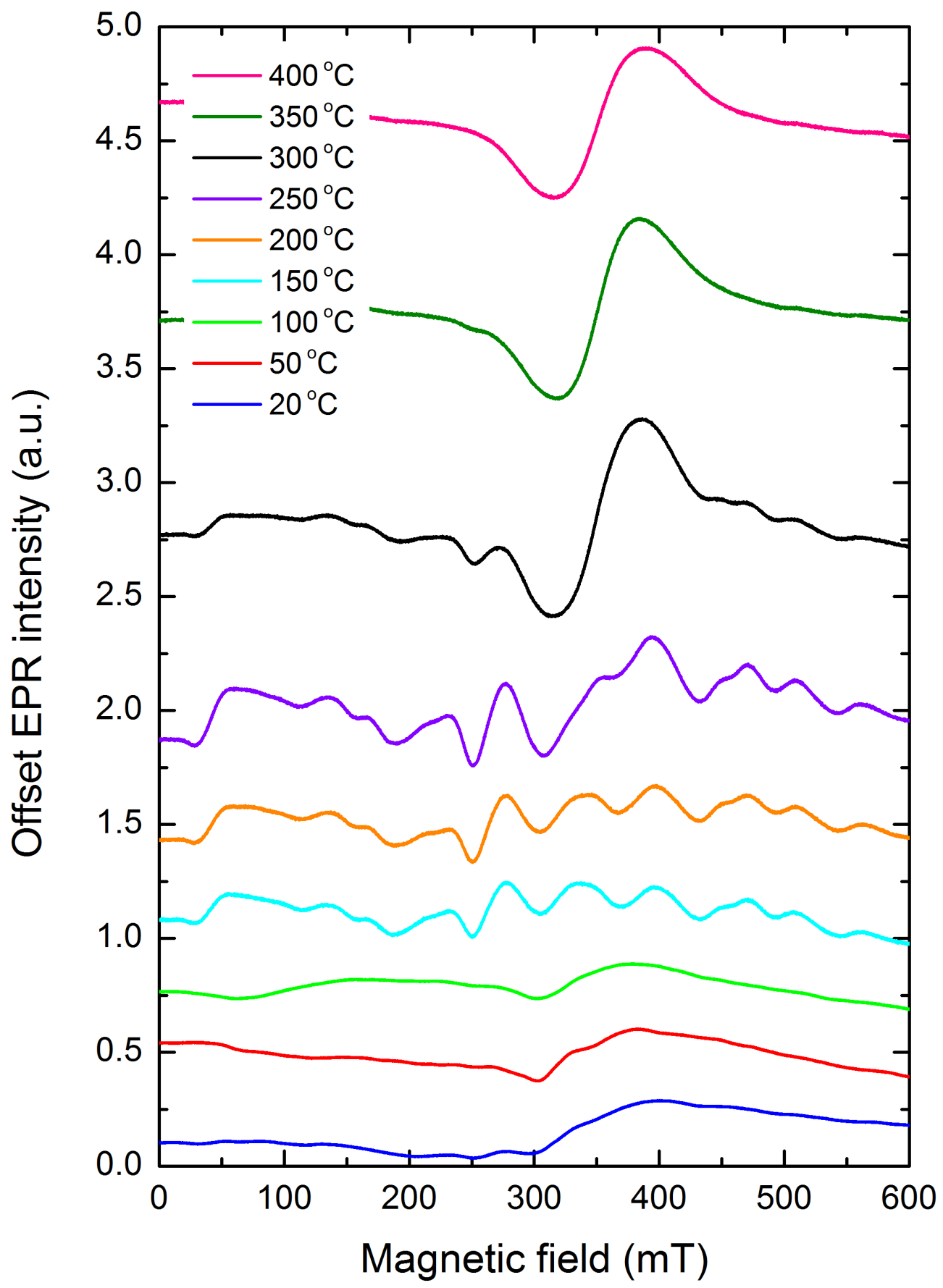

Figure 5.26: Electron paramagnetic resonance spectra for $\mathrm{CsBr}: \mathrm{Eu}^{2+}$ imaging plates annealed at the temperatures shown. Spectra were measured at room temperature with a microwave frequency of $9.52 \mathrm{GHz}$, modulation amplitude of $5 \mathrm{~V}$ and a $6 \mathrm{~dB}$ power level.

150 and $250{ }^{\circ} \mathrm{C}$ is the same as reported by Loncke et al. [33], and assigned to a $\mathrm{Eu}^{2+}-\mathrm{Cs}_{\mathrm{v}}-\mathrm{Cs}_{\mathrm{v}}\left(\mathrm{H}_{2} \mathrm{O}\right)$ site, a simulation of the $9.5 \mathrm{GHz}$ EPR spectrum for a powder has been performed. A direct comparison of the spectra is impossible, as the single crystal and powder spectra reported by Loncke et al. [33] were 
recorded at $\mathrm{Q}$ band $(\sim 35 \mathrm{GHz})$ and are very different from those expected at $\mathrm{X}$ band $(\sim 9 \mathrm{GHz})$.

The spin Hamiltonian for $\mathrm{Eu}^{2+}$ ions at a tetragonal symmetry site is given by,

$$
\mathscr{H}=\mu_{B} \mathbf{B} \cdot \mathbf{g} \cdot \mathbf{S}+B_{2}^{0} O_{2}^{0}+B_{4}^{0} O_{4}^{0}+B_{4}^{4} O_{4}^{4}+\sum_{m} B_{6}^{m} O_{6}^{m}+\sum_{i} A_{i} \mathbf{I}_{i} \cdot \mathbf{S},
$$

where $S=\frac{7}{2}$ is the electronic spin of $\mathrm{Eu}^{2+}$ and $I_{i}$ is the nuclear spin of the two isotopes, ${ }^{157} \mathrm{Eu}$ and ${ }^{153} \mathrm{Eu}$, both with $I=\frac{5}{2}$. The terms $B_{l}^{m} O_{l}^{m}$ represent the zero-field splitting (crystal field term) for tetragonal symmetry, and following Loncke et al., we neglect the very small $B_{6}^{m} O_{6}^{m}$ term. For an isolated $\mathrm{Eu}^{2+}$ ion there would be no zero-field splitting due to the half-filled $4 f$ shell being spherically symmetric. However, in a crystal environment the crystal field can cause admixtures of higher electronic wavefunctions (such as $5 d$ ) into the ground state and lead to zero-field splittings. The $O_{l}^{m}$ operators are the Steven's operator equivalents, as defined in Abragam and Bleaney [68] for example. The $g$ and $A$ tensors are assumed to be isotropic and so are represented by simple scalars.

For each europium isotope there are $(2 S+1)(2 I+1)$ energy levels, and EPR transitions within them are governed by the (high field) selection rule, $\Delta M_{S}=$ $\pm 1, \Delta M_{I}=0$, giving a very complicated spectrum. In addition, for a powder, the spectrum needs to be averaged over all possible relative orientations of magnetic field and symmetry axis orientations.

Table 5.2: Spin Hamiltonian parameters used to simulate $X$ band EPR spectra [35].

\begin{tabular}{cc}
\hline Parameter & Value \\
\hline$g$ & 1.9913 \\
$B_{2}^{0}$ & $755.4 \mathrm{MHz}$ \\
$B_{4}^{0}$ & $0.27 \mathrm{MHz}$ \\
$B_{4}^{4}$ & $-3.48 \mathrm{MHz}$ \\
$A\left({ }^{157} \mathrm{Eu}\right)$ & $-41.9 \mathrm{MHz}$ \\
$A\left({ }^{153} \mathrm{Eu}\right)$ & $-93.5 \mathrm{MHz}$ \\
$h \nu$ & $9.52 \mathrm{GHz}$ \\
\hline
\end{tabular}




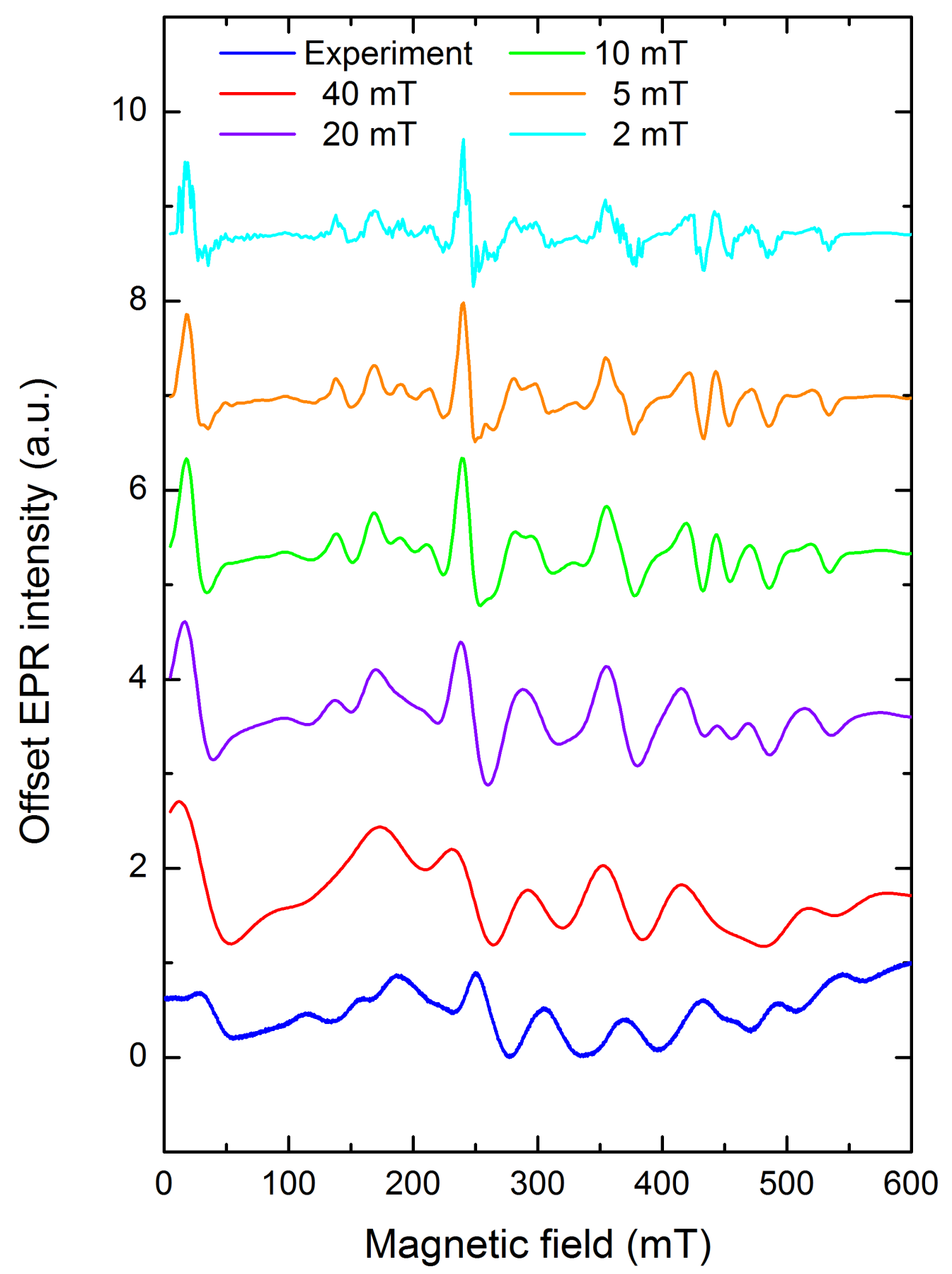

Figure 5.27: Room temperature 9.52 GHz EPR spectrum of a pressed imaging plate annealed at $150{ }^{\circ} \mathrm{C}$ compared to simulated spectra characteristic of the active PSL site using the parameters given in table 5.2, and various linewidths as shown.

In this work, the EASYSPIN package [69] has been used to simulate the expected $\mathrm{X}$ band EPR spectrum. The required spin Hamiltonian parameters, taken from [35, 36] are given in table 5.2 .

The powder spectrum was generated from a net of relative orientations with 
the mesh size being five degrees. A Gaussian lineshape is assumed. Vrielinck et al. [36] published spectra which showed a linewidth of approximately $12 \mathrm{mT}$, sufficiently large that hyperfine structure could not be resolved. In the present case, the broadening effects of lattice strain, dislocations and point defects may give rise to an even larger linewidth, and so the simulation has been performed for a range of linewidths, $2-40 \mathrm{mT}$, as shown in figure 5.27 . Figure 5.27 shows that the simulated spectra for linewidths in the range 20 - $40 \mathrm{mT}$ show a very good general correlation with the observed spectra for an imaging plate annealed at $150{ }^{\circ} \mathrm{C}$. This confirms that the PSL active site generated in this work is identical to that reported by Vrielinck et al. [36]. The agreement is not perfect, but given the complexity of the calculation, the sensitivity to some parameters such as the linewidth, and the omission of significant spectral behaviour such as the angular dependence of linewidths, the agreements is considered to be very satisfactory.

\subsection{Method 4: Milling, annealing and pressing}

As both hydrating and annealing the imaging plates increases the PSL intensity, investigations into simultaneous annealing and hydrating were made. Currently, only imaging plates made by hydrating and annealing as a powder have been tested. Hydrating at elevated temperatures in room air was achieved using a hotplate, with the powder encased in aluminium foil. Hydrating in saturated air at elevated temperatures was achieved using a quartz tube placed half in a furnace, with a small container of water at the room temperature end.

\subsubsection{Room air hydration}

A powder mixture of $\mathrm{CsBr}$ and $\mathrm{EuBr}_{2}$ was hydrated in room air $(30 \% \mathrm{RH})$ at temperatures ranging from room temperature to $300{ }^{\circ} \mathrm{C}$ for 15 minutes. The powders were then pressed into pellets as described previously. The optical 


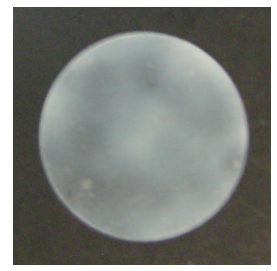

(a) $20^{\circ} \mathrm{C}$

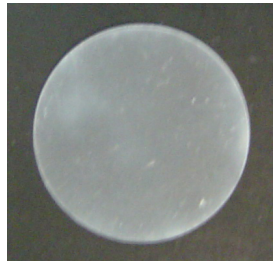

(b) $50{ }^{\circ} \mathrm{C}$

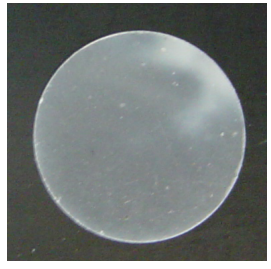

(c) $100{ }^{\circ} \mathrm{C}$

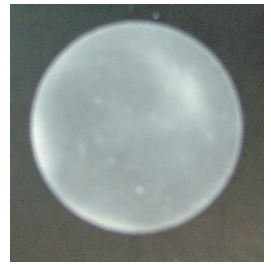

(d) $150{ }^{\circ} \mathrm{C}$

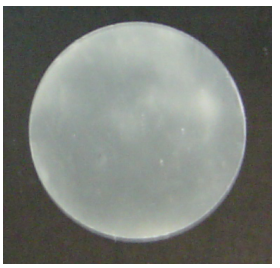

(e) $200{ }^{\circ} \mathrm{C}$

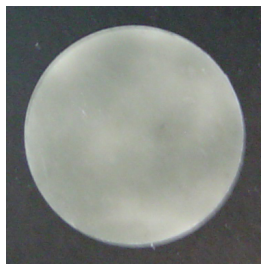

(f) $250{ }^{\circ} \mathrm{C}$

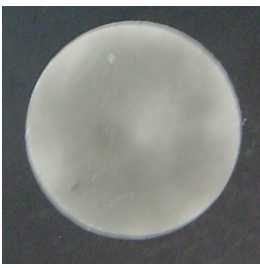

(g) $300{ }^{\circ} \mathrm{C}$

Figure 5.28: Optical appearance of $\mathrm{CsBr}: \mathrm{Eu}^{2+}$ imaging plates hydrated in air at the temperatures shown.

appearance of these imaging plates are shown in figure 5.28, Consistent with hydrating as a powder, these imaging plates are inhomogeneous and show cloudy and transparent regions. For temperatures of $250{ }^{\circ} \mathrm{C}$ and greater the imaging plates show a slight change in colour from white to brown.

The UV-Vis extinction and transmission coefficient as a function of hydration temperature for these imaging plates are shown in figure 5.29. As the powder is hydrated from room temperature (approximately $20^{\circ} \mathrm{C}$ ) up to $100{ }^{\circ} \mathrm{C}$ the trans-

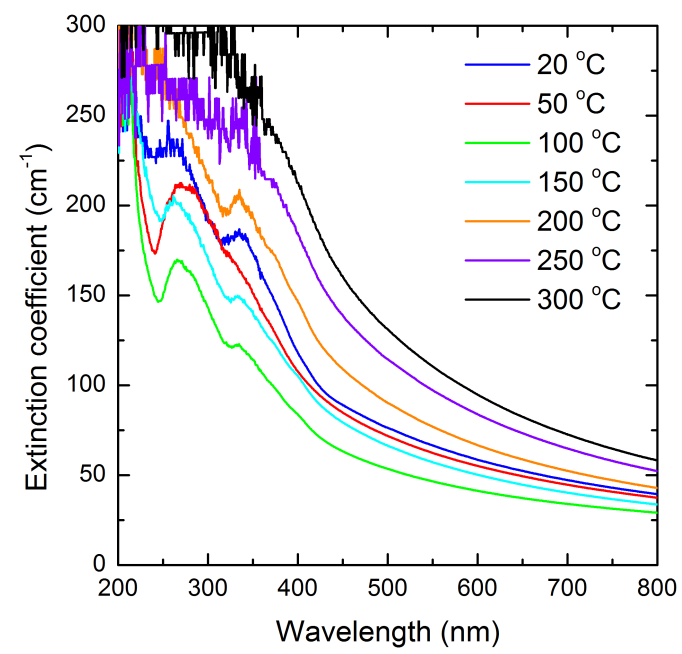

(a)

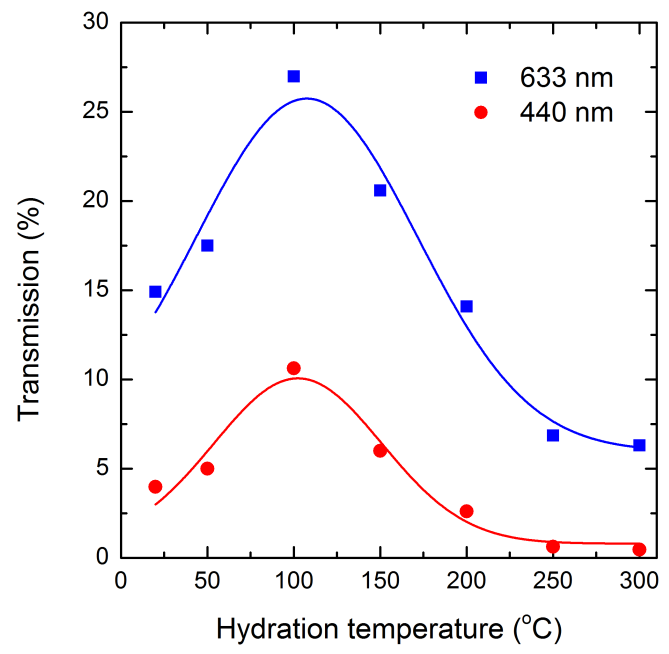

(b)

Figure 5.29: (a) UV-Vis extinction spectra for $\mathrm{CsBr}_{\mathrm{Eu}}{ }^{2+}$ imaging plates hydrated in room air for the temperatures shown, and (b) transmission versus hydration temperature at $633 \mathrm{~nm}$ (blue squares) and $440 \mathrm{~nm}$ (red circles). 
mission of the imaging plates increases. For hydration temperatures greater than $100{ }^{\circ} \mathrm{C}$ the transmission decreases, as the cloudy regions dominate the transparent ones and the measurement is an average over the beam area. The extinction spectra (figure 5.29(a)) again show a scattering background with $\mathrm{e}_{\mathrm{g}}$ and $t_{2 g}$ peaks superimposed. The room temperature processed imaging plate has the $e_{g}$ and $t_{2 g}$ peaks in the locations associated with the PSL active site. Annealing at $50{ }^{\circ} \mathrm{C}$, these peaks are shifted and correlated with the $\mathrm{EuBr}_{2} \cdot 1 \mathrm{H}_{2} \mathrm{O}$ positions. A further increase in hydration temperature up to $150{ }^{\circ} \mathrm{C}$ causes these peaks to shift back to the positions corresponding to the PSL active site. Beyond $150{ }^{\circ} \mathrm{C}$ the peaks cannot be distinguished from the noise. The increase in transmission is due to a decrease in the water content of the imaging plates, as will be seen in the IR spectra.

Infrared spectra for these imaging plates are shown in figure 5.30. The initial sample hydrated at room temperature for 15 minutes still shows evidence of $\mathrm{EuBr}_{2} \cdot 1 \mathrm{H}_{2} \mathrm{O}$. This is surprising as previously (section 5.2) it was shown that for 5 minutes hydration in air with $40 \% \mathrm{RH}$, most has been converted to the PSL active site. This suggests the hydration factor is strongly dependent on the relative humidity and even a $10 \%$ difference can have a large effect. Hydrating

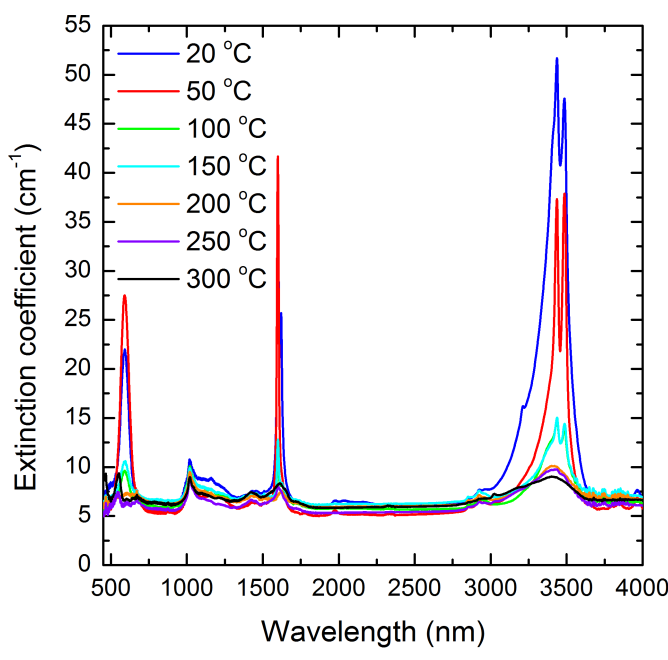

(a)

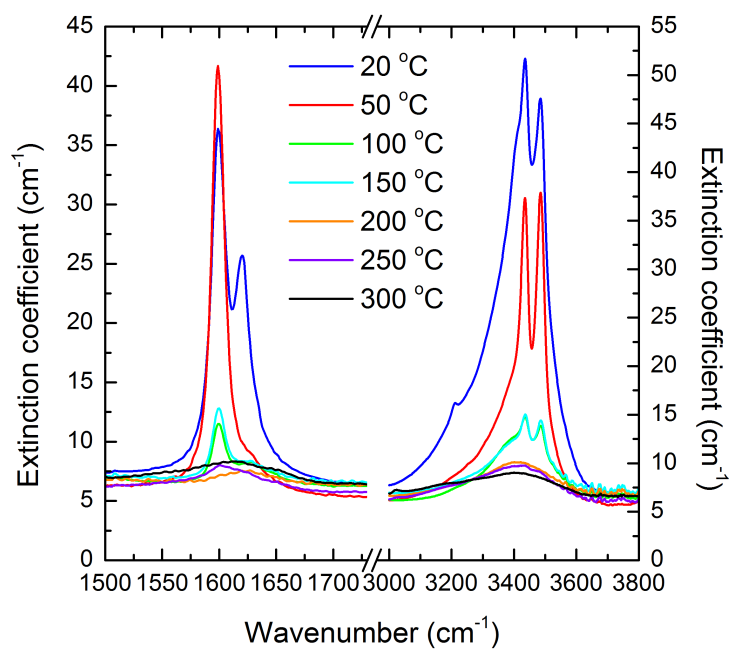

(b)

Figure 5.30: (a) IR spectra for $\mathrm{CsBr}: \mathrm{Eu}^{2+}$ imaging plates in room air at the temperatures shown. (b) Enlarged view of the $\nu_{1}, \nu_{3}$ region (right) and $\nu_{2}$ region (left). 


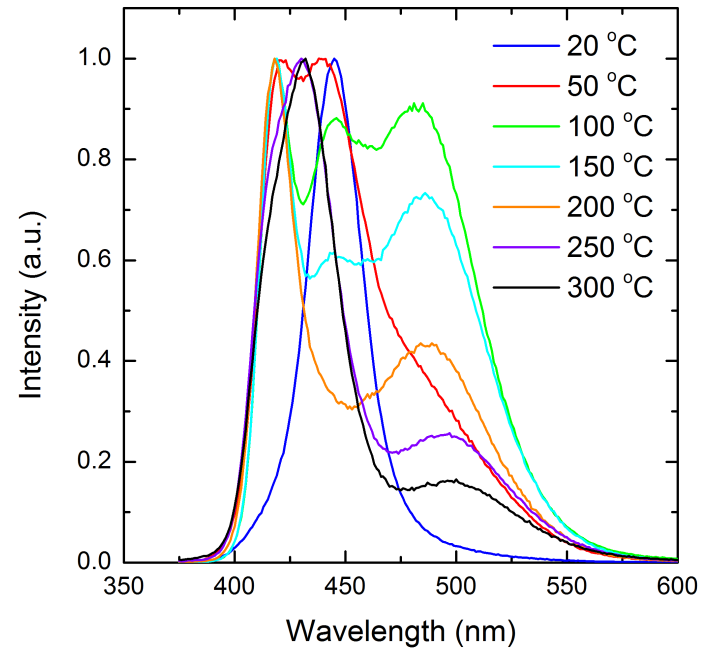

(a)

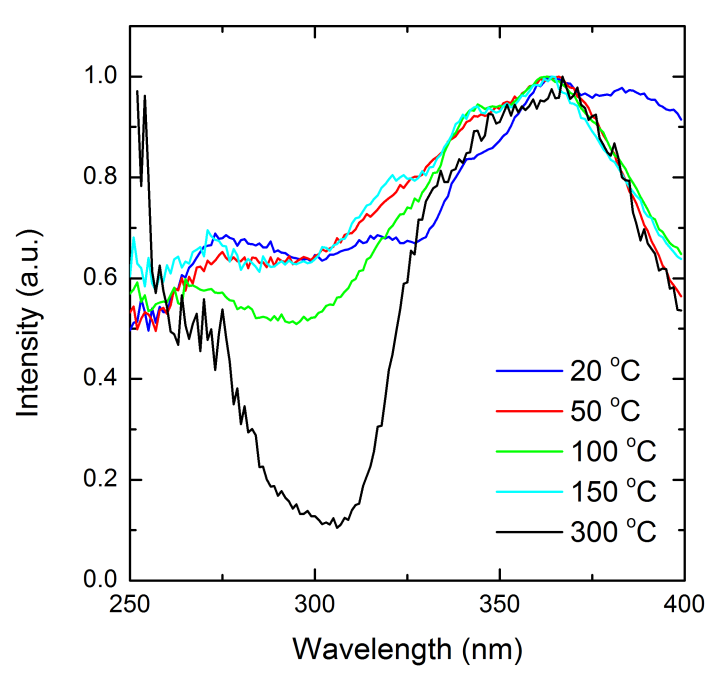

(b)

Figure 5.31: PL (a) emission and (b) excitation spectra of $\mathrm{CsBr}: \mathrm{Eu}^{2+}$ imaging plates hydrated in room air at the temperatures shown.

at $50{ }^{\circ} \mathrm{C}$ causes the sharp peaks due to $\mathrm{EuBr}_{2} \cdot 1 \mathrm{H}_{2} \mathrm{O}$ to increase, although the total water content decreases. The $\mathrm{EuBr}_{2} \cdot 1 \mathrm{H}_{2} \mathrm{O}$ peaks are still strong at hydration temperatures up to $150{ }^{\circ} \mathrm{C}$, but any further increase in temperature sees the $\mathrm{EuBr}_{2} \cdot 1 \mathrm{H}_{2} \mathrm{O}$ peaks disappear along with a decrease in water content.

The photoluminescence emission spectra (figure 5.31) do not show the same trends as expected from the IR spectra. Initially, the spectrum for the room temperature annealed sample shows a single emission peak at $440 \mathrm{~nm}$ corresponding to the $\mathrm{Eu}^{2+}$ ion in the PSL active site. From the IR curve shown in figure 5.30, one would expect the curve to show at least two peaks corresponding to both the $\mathrm{Eu}^{2+}$ emission and the $\mathrm{EuBr}_{2}$ emission. The reason for this is unclear. As the hydration temperature increases, the PL curves show the three peaked emission as described earlier. At $50{ }^{\circ} \mathrm{C}$ the emission curve is dominated by the $440 \mathrm{~nm} \mathrm{Eu}{ }^{2+}$ and $\mathrm{EuBr}_{2}$ emissions. As the temperature increases up until $150{ }^{\circ} \mathrm{C}$, the PL shows a three peaked emission curve with an increasing emission band at $485 \mathrm{~nm}$. At annealing temperatures of $200{ }^{\circ} \mathrm{C}$ or greater the PL spectra consist mainly of the $420 \mathrm{~nm}$ and $485 \mathrm{~nm}$ emissions, with almost no contribution from the $440 \mathrm{~nm} \mathrm{Eu}^{2+}$ emission. The excitation spectra for these imaging plates are shown in figure 5.31(b), For room temperature hydration, a small peak at 


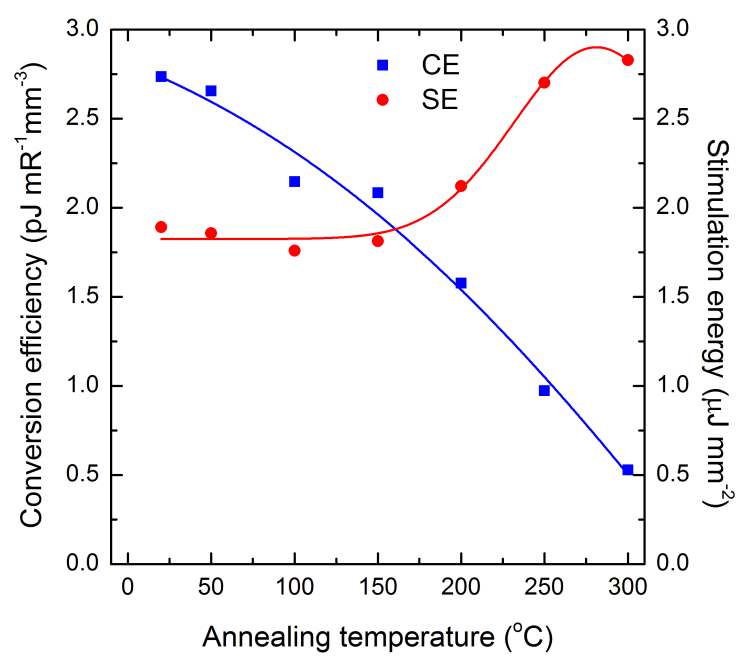

Figure 5.32: CE (blue squares) and SE (red circles) for $\mathrm{CsBr}: \mathrm{Eu}^{2+}$ imaging plates hydrated as a powder in room air as a function of hydration temperature.

$340 \mathrm{~nm}$ is seen corresponding to $\mathrm{EuBr}_{2}$. Hydration temperatures between 50 and $150{ }^{\circ} \mathrm{C}$ all show similar excitation spectra, which are characteristic for the corresponding emission spectra. At $300^{\circ} \mathrm{C}$ the excitation spectrum has shifted to slightly lower wavelengths (as seen in figure 5.20(b)), and is correlated with a low CE.

These trends are also seen in the conversion efficiency and stimulation energy measurements shown in figure 5.32. As the annealing temperature is increased the CE decreases and the SE increases. The decrease in CE correlates with the decrease in free water seen in the IR spectra (figure 5.30), and is anti-correlated with the presence of the $\mathrm{Eu}^{2+}-\mathrm{Cs}_{\mathrm{v}}$ agglomerate PL band at $485 \mathrm{~nm}$.

\subsubsection{Saturated air hydration}

Powders were also hydrated at various temperatures in saturated air (100\% RH) for 15 minutes before being pressed into imaging plates. The optical appearance of these imaging plates are shown in figure 5.33. Compared to the previously discussed case these imaging plates are much less transparent and have large areas which have clouded over. These imaging plates also show a significant colour change for temperatures greater than $200{ }^{\circ} \mathrm{C}$.

The UV-Vis extinction spectra for the different hydration temperatures are 


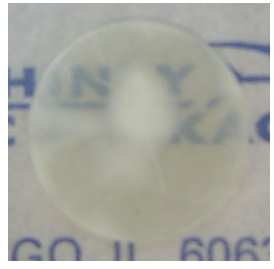

(a) $20^{\circ} \mathrm{C}$

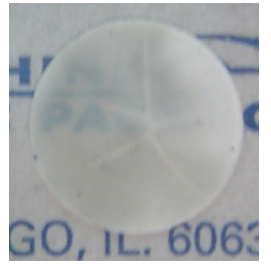

(b) $50{ }^{\circ} \mathrm{C}$

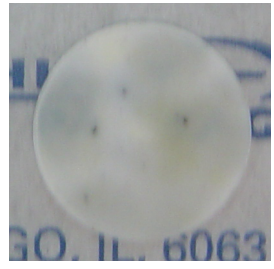

(c) $100{ }^{\circ} \mathrm{C}$

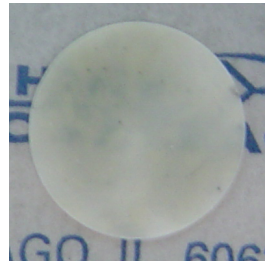

(d) $150{ }^{\circ} \mathrm{C}$

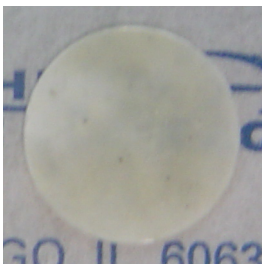

(e) $200{ }^{\circ} \mathrm{C}$

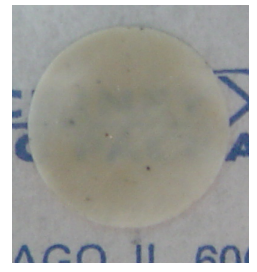

(f) $250{ }^{\circ} \mathrm{C}$

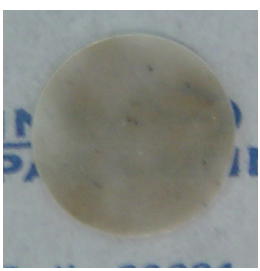

(g) $300{ }^{\circ} \mathrm{C}$

Figure 5.33: Optical appearance of imaging plates made by hydrating the powder in saturated air at the temperatures shown.

shown in figure 5.34(a), In these spectra the $e_{g}$ and $t_{2 g}$ peaks are not visible under the high noise level caused by scattering in the imaging plates. However, the general trend for the $t_{2 g}$ peak can be seen by the onset wavelength at which the spectra deviate from simply scattering behaviour. This suggests that as the temperature is increased the $t_{2 g}$ peak shifts to higher wavelengths. The transmission coefficient (figure 5.34(a)) at both $633 \mathrm{~nm}$ and $440 \mathrm{~nm}$ de-

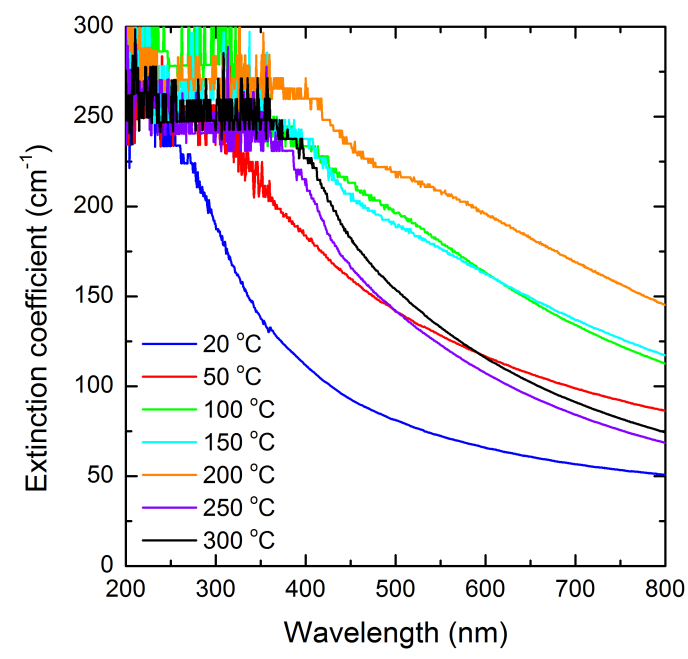

(a)

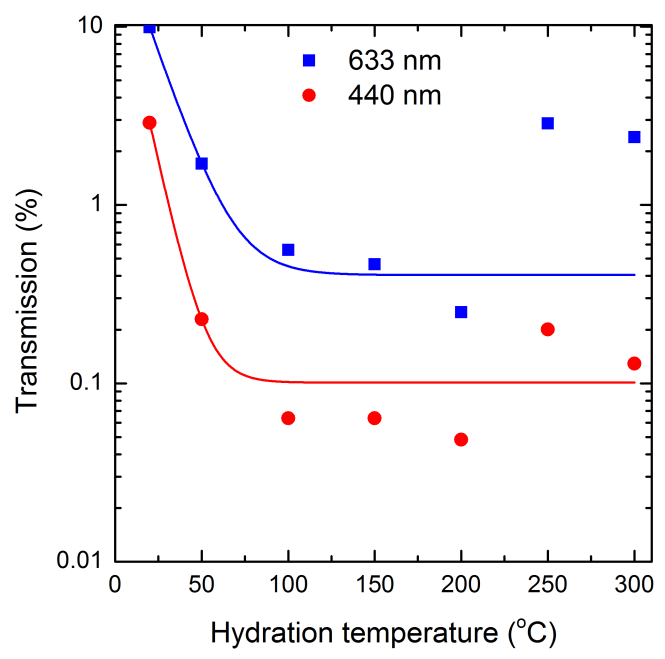

(b)

Figure 5.34: (a) UV-Vis extinction spectra for imaging plates made by hydrating the powder in saturated air at the temperatures shown, and (b) transmission at $633 \mathrm{~nm}$ (blue squares) and $440 \mathrm{~nm}$ (red circles) as a function of hydration temperature. 


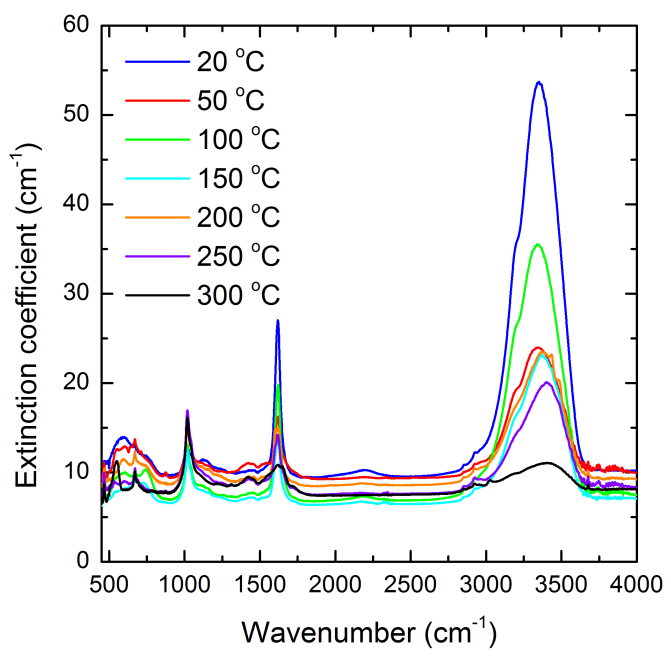

(a)

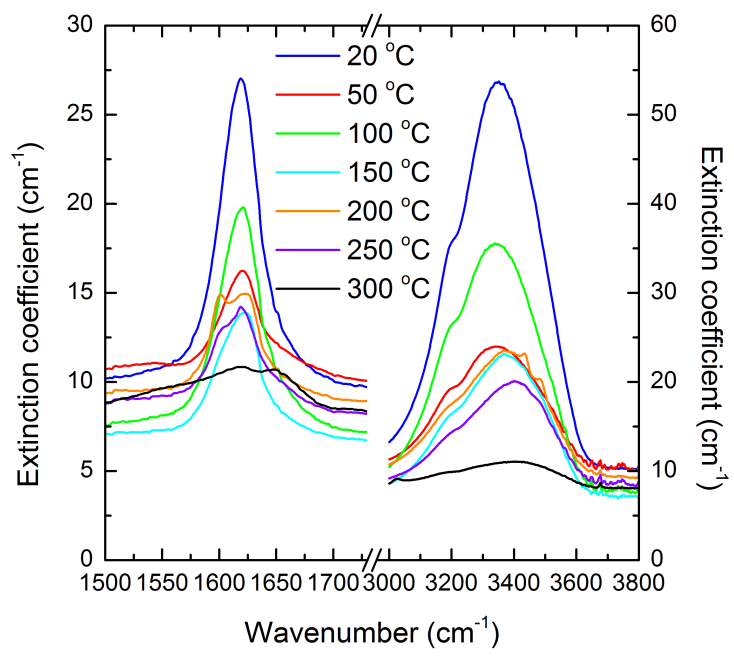

(b)

Figure 5.35: IR spectra for imaging plates made by hydrating in saturated air at the temperatures shown. (b) Enlarged view of the $\nu_{1}, \nu_{3}$ region (right) and $\nu_{2}$ region (left).

creases with increasing hydration temperature. The transmission coefficients at temperatures of 250 and $300{ }^{\circ} \mathrm{C}$ deviate from the general trend, due to those imaging plates having larger transparent areas as can be seen particularly in figure 5.33(f). These imaging plates show a much lower transmission than those hydrated in room air as is expected.

The IR spectra for these imaging plates are shown in figure 5.35. The imaging plate hydrated at room temperature shows an IR spectrum consistent with those shown for the high humidity hydration curves shown previously. As the annealing temperature is increased up to $150{ }^{\circ} \mathrm{C}$, the water content of the imaging plates generally decreases while the spectral shape remains the same. Annealing at $50{ }^{\circ} \mathrm{C}$ shows a lower water content than at $100{ }^{\circ} \mathrm{C}$. At annealing temperatures of 200 and $250{ }^{\circ} \mathrm{C}$, minor peaks corresponding to $\mathrm{EuBr}_{2} \cdot 1 \mathrm{H}_{2} \mathrm{O}$ appear in both the $\nu_{1}, \nu_{3}$ stretching and $\nu_{2}$ bending vibrations.

The photoluminescence emission spectra for these imaging plates are shown in figure 5.36(a). Imaging plates hydrated at temperatures up to and including $150{ }^{\circ} \mathrm{C}$ have a single band emission at $440 \mathrm{~nm}$. When hydrated at $200{ }^{\circ} \mathrm{C}$, the PL spectrum has a small sideband at higher wavelengths, which corresponds to a peak at around $500 \mathrm{~nm}$ for imaging plates hydrated at 250 and $300{ }^{\circ} \mathrm{C}$. At these 


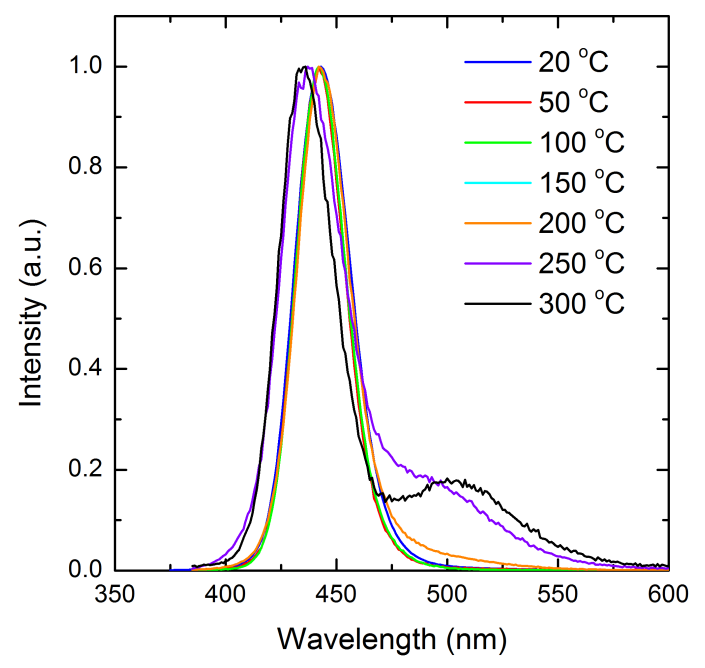

(a)

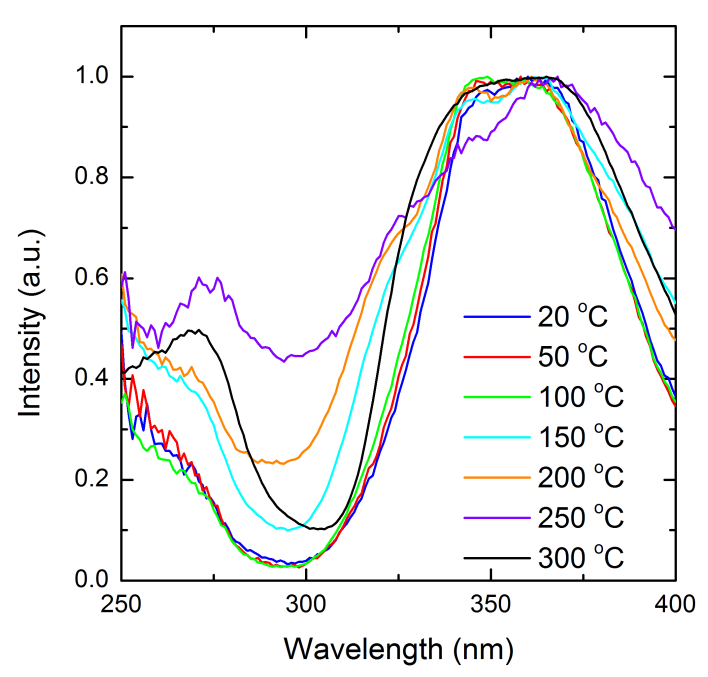

(b)

Figure 5.36: PL (a) emission and (b) spectra for imaging plates made by hydrating the powder in saturated air at the temperatures shown.

higher hydration temperatures the main peak of the emission has also shifted to lower wavelengths (435 nm). The change in PL is likely associated with a new $\mathrm{Eu}^{2+}$ site forming, and correlates with a decrease in the PSL intensity. The PL excitation spectra (figure 5.36(b)) are the same for hydration temperatures between 20 and $200{ }^{\circ} \mathrm{C}$. At $250{ }^{\circ} \mathrm{C}$ the spectrum shows a decrease in the $340 \mathrm{~nm}$ peak, which was previously attributed to $\mathrm{EuBr}_{2}$.

The CE and SE for these imaging plates (figure 5.37) show a trend similar to the PL spectra. Increasing the hydration temperature increases the CE until a

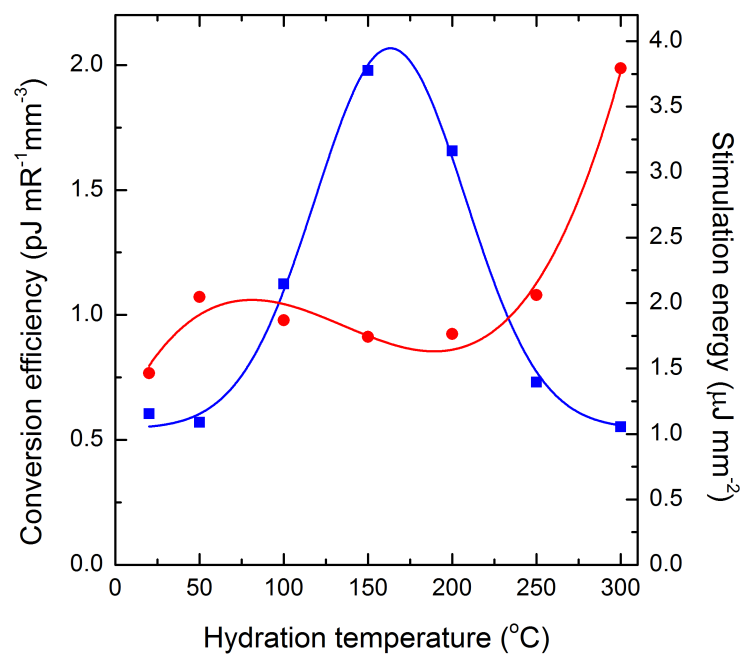

Figure 5.37: CE (blue squares) and SE (red circles) for CsBr:Eu ${ }^{2+}$ imaging plates hydrated as a powder in saturated air . 
maximum occurs at around $150{ }^{\circ} \mathrm{C}$, with the SE remaining relatively constant. Above $150{ }^{\circ} \mathrm{C}$ the $\mathrm{CE}$ decreases and the SE increases, meaning both the emission for a given $\mathrm{X}$-ray dose has decreased and a larger stimulation energy is required to read out the image. The decrease in CE correlates with the IR and PL spectra given here, and also follows a similar trend to the results given for the imaging plates annealed under vacuum (section 5.4).

\subsection{Method 5: Sintering, milling, pressing and hy- drating}

CsBr:Eu ${ }^{2+}$ imaging plates were made by sintering the mixed $\mathrm{CsBr}$ and $\mathrm{EuBr}_{2}$ powders before milling to a grain size of $25 \mu \mathrm{m}$, and then pressing into imaging plates. The idea behind sintering is to promote diffusion of $\mathrm{Eu}^{2+}$ ions into the $\mathrm{CsBr}$ lattice. The $\mathrm{CsBr}$ and $\mathrm{EuBr}_{2}$ powders were mixed and placed into a glassy carbon boat, in a tube furnace. The temperature was raised to $200{ }^{\circ} \mathrm{C}$ at $3{ }^{\circ} \mathrm{C}$ per hour and held for 2 hours under a constant high purity argon flow. In the literature a much higher temperature of $600^{\circ} \mathrm{C}$ [29] was used (just below the melting point of $636{ }^{\circ} \mathrm{C}$ for $\mathrm{CsBr}$ ), but here this resulted in a grey powder and reduced the optical quality of the imaging plates. Subsequently, the imaging plates were subjected to a hydration treatment in saturated air at room temperature.

The optical appearance of the pre-sintered imaging plates are shown in figure 5.38. As the hydration time is increased the imaging plates become less trans-

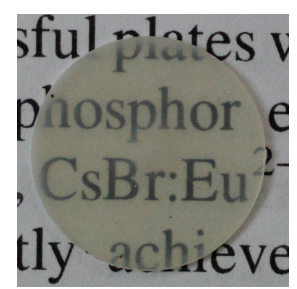

(a) $0 \mathrm{~min}$

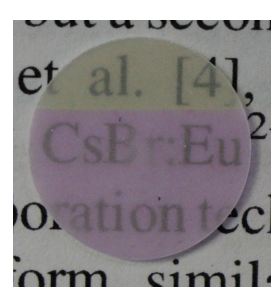

(b) $30 \mathrm{~s}$

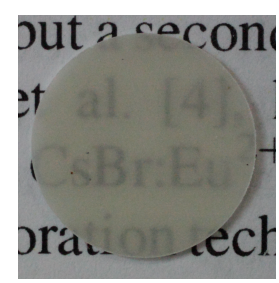

(c) $1 \mathrm{~min}$

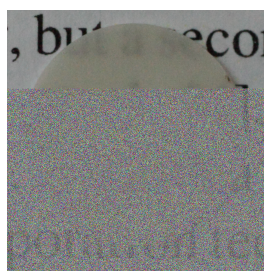

(d) $2 \mathrm{~min}$

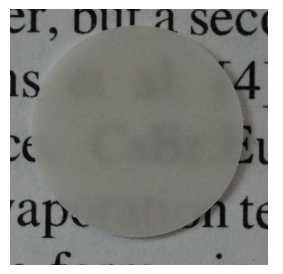

(e) $5 \mathrm{~min}$

Figure 5.38: Optical appearance of pre-sintered $\mathrm{CsBr}: \mathrm{Eu}^{2+}$ imaging plates hydrated in saturated air for the times shown. 
parent, as found in section 5.2. The reduction in transparency was previously found to be caused by a recrystallisation process.

The UV-Vis extinction spectra and transmission coefficient for the various hydration times are shown in figure 5.39. The extinction spectrum for the as-made (not hydrated) imaging plate shows a very flat baseline, corresponding to high transmission, with the $e_{2 g}$ and $t_{2 g}$ peaks evident at low wavelengths. The $e_{g}$ and $t_{2 g}$ peaks in this case are much sharper than hydrated non-sintered imaging plates and are characteristic of $\mathrm{Eu}^{2+}$ ions in the PSL active site. As the hydration time is increased the background of the extinction curve increases (increased scattering) and the $\mathrm{e}_{2 g}$ and $\mathrm{t}_{2 \mathrm{~g}}$ peaks can no longer be distinguished above the noise level. The transmission of 633 and $440 \mathrm{~nm}$ light through the imaging plate (figure 5.39(b)) decreases with increasing hydration time, as expected from previous results. The initial transmission coefficient is approximately $33 \%$ at $633 \mathrm{~nm}$ and $15 \%$ at $440 \mathrm{~nm}$ as compared to $35 \%$ and $11 \%$ for the non-sintered imaging plates. The transmission coefficient at 5 minutes hydration time, at 0.2 $\%$ for both wavelengths, is slightly higher than for the non-sintered imaging plates.

Infrared spectra for the pre-sintered hydrated imaging plates are shown in

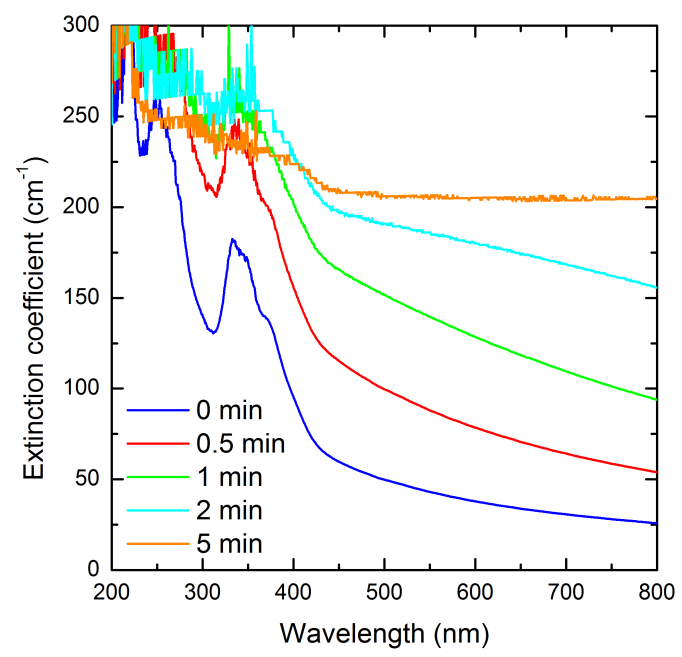

(a)

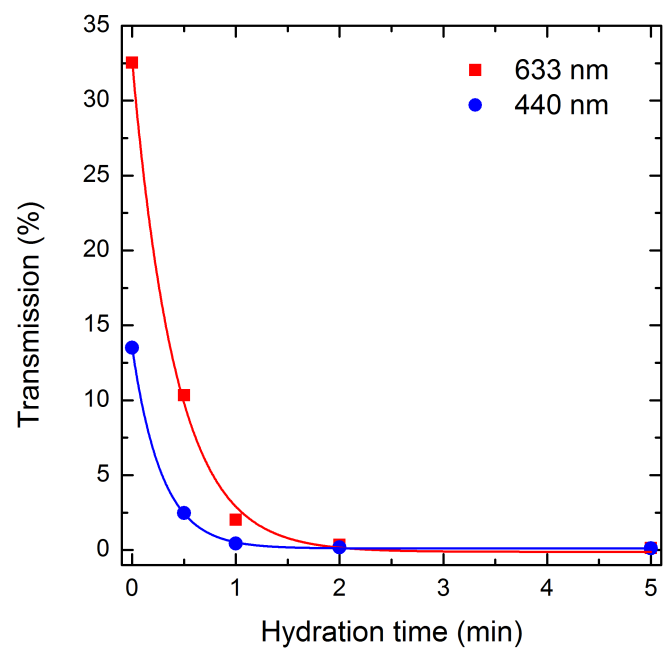

(b)

Figure 5.39: (a) UV-Vis extinction spectra for pre-sintered imaging plates hydrated in saturated air for the times shown, and (b) transmission at $633 \mathrm{~nm}$ (blue squares) and $440 \mathrm{~nm}$ (red circles) as a function of hydration time. 


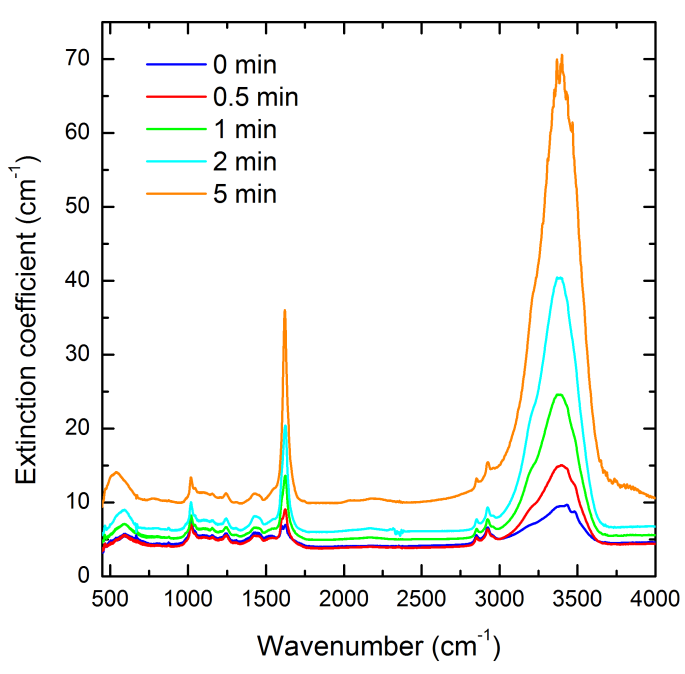

(a)

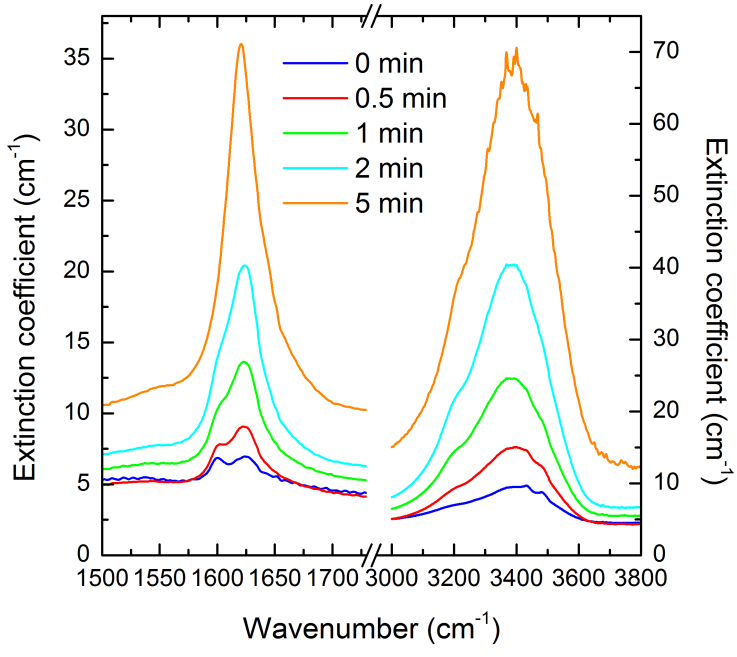

(b)

Figure 5.40: IR spectra for pre-sintered $\mathrm{CsBr}: \mathrm{Eu}^{2+}$ imaging plates hydrated in saturated air for the times shown. (b) Enlarged view of the $\nu_{1}, \nu_{3}$ region (right) and $\nu_{2}$ region (left).

figure 5.40. The as-made, non-hydrated imaging plate shows evidence of $\mathrm{EuBr}_{2} \cdot 1 \mathrm{H}_{2} \mathrm{O}$, particularly in the $\nu_{2}$ bending vibration. The general trend with increasing hydration time is the same as for the non-sintered plates. That is, as the hydration time increases the water content increases and the $\mathrm{EuBr}_{2} \cdot 1 \mathrm{H}_{2} \mathrm{O}$ is converted into the PSL active site. The uptake of water by the pre-sintered imaging plates for each hydration time is less compared to the non-sintered imaging plates.

The PL emission and excitation spectra for the various hydration times are shown in figure 5.41. The spectrum for the as-made imaging plate has a main emission peak at $440 \mathrm{~nm}$, corresponding to the PSL active site, and a broad band at approximately $485 \mathrm{~nm}$ due to $\mathrm{Eu}^{2+}-\mathrm{Cs}_{\mathrm{v}}$ agglomerates. Additionally there is a small side band at lower wavelengths associated with $\mathrm{EuBr}_{2}$ emission. As the hydration time is increased both side bands are reduced in amplitude relative to the $440 \mathrm{~nm}$ band and then disappear for a hydration time of 5 minutes. At 5 minutes hydration only the emission from the PSL active site is seen. The excitation spectra for the pre-sintered imaging plates shows two main bands, occurring at approximately $270 \mathrm{~nm}$ for the $\mathrm{e}_{\mathrm{g}}$ transition and $360 \mathrm{~nm}$ for the $t_{2 g}$. The $t_{2 g}$ band shows further splitting where the $320 \mathrm{~nm}$ and $345 \mathrm{~nm}$ peaks 


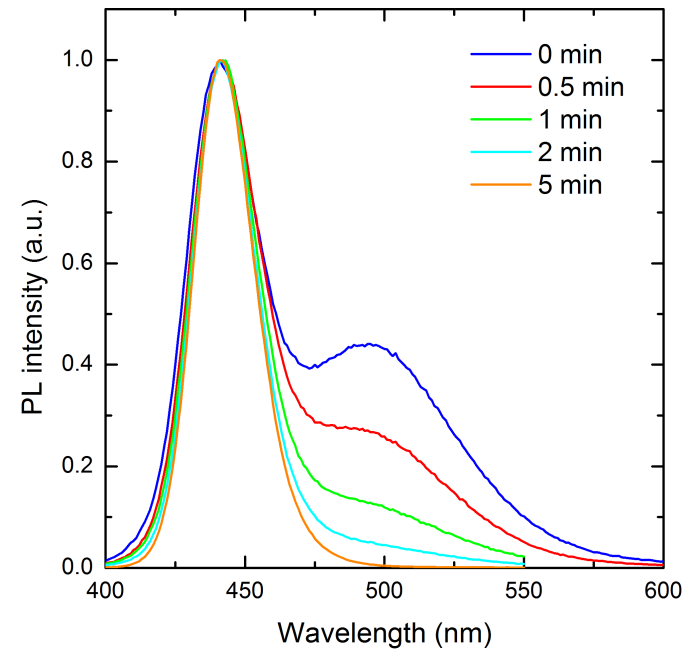

(a)

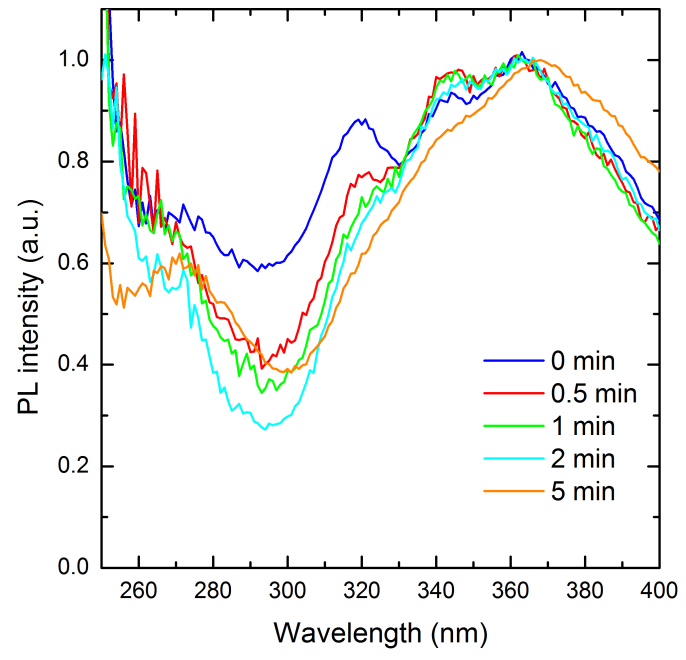

(b)

Figure 5.41: PL (a) emission and (b) excitation spectra for pre-sintered CsBr:Eu ${ }^{2+}$ imaging plates hydrated in saturated air for the times shown.

are assumed to be related to $\mathrm{EuBr}_{2}$ or $\mathrm{EuBr}_{2} \cdot 1 \mathrm{H}_{2} \mathrm{O}$. As the hydration time is increased the peaks at 320 and $345 \mathrm{~nm}$ decrease which is associated with the generation of the PSL active site.

The PSL performance of these imaging plates is shown in figure 5.42. The conversion efficiency increases markedly with increasing hydration time, which is correlated with a decrease in the $485 \mathrm{~nm}$ PL emission and formation of the PSL active site. The stimulation energy decreases slightly with increasing hydration time as was also found for the non-sintered imaging plates. For 5 minutes

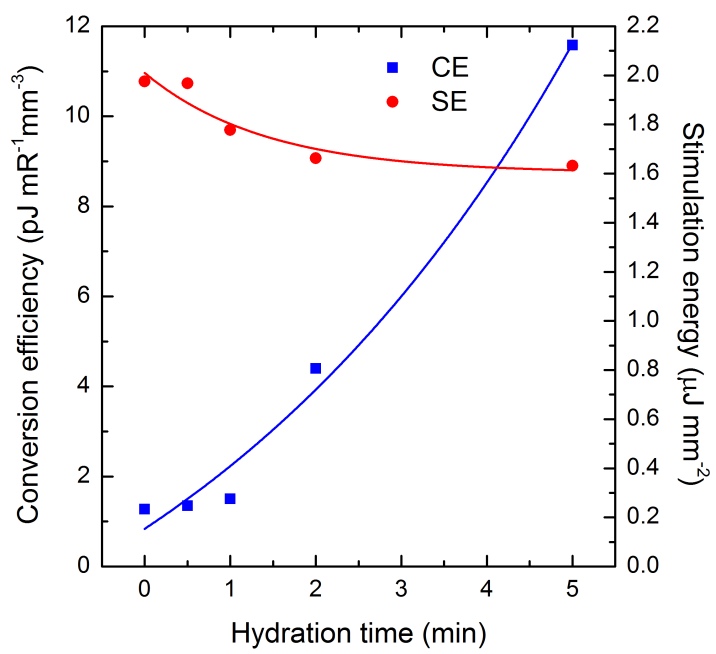

Figure 5.42: CE (blue squares) and SE (red circles) for pre-sintered imaging plates hydrated in saturated air for the times shown. 


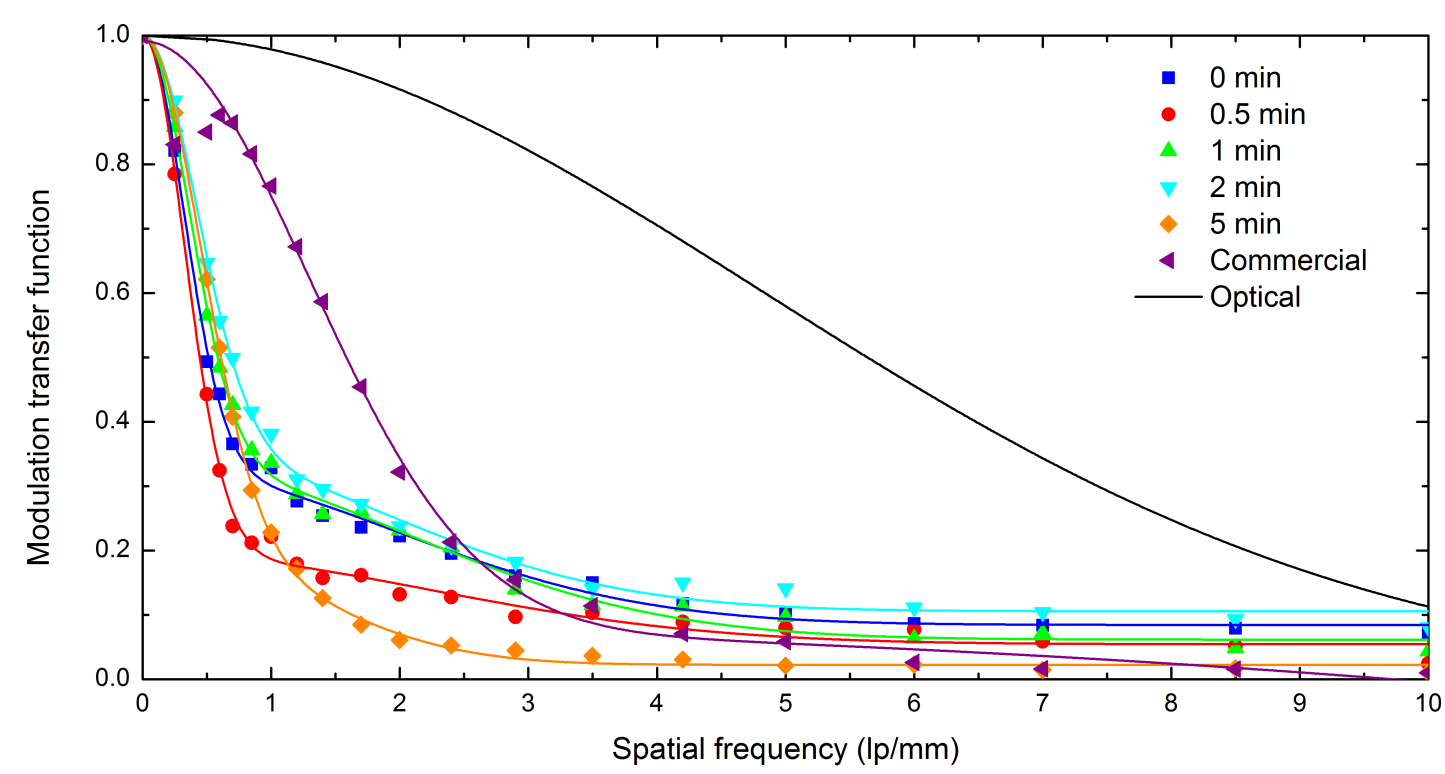

Figure 5.43: Optical and X-ray MTFs for the camera system and various presintered CsBr:Eu ${ }^{2+}$ imaging plates. The data points have been fitted with the sum of two Gaussians.

hydration the $\mathrm{CE}$ is $11.7 \mathrm{pJ} \mathrm{mR}^{-1} \mathrm{~mm}^{-3}$ compared to $5.1 \mathrm{pJ} \mathrm{mR} \mathrm{mm}^{-1} \mathrm{~mm}^{-3}$ calculated for the commercial imaging plate. This is higher than the CE found for the 5 minute hydrated non-sintered imaging plate.

The resolution in terms of the modulation transfer function of the pre-sintered imaging plates for various hydration times is shown figure 5.43. Compared to the commercial imaging plate, these imaging plates again show a bimodal character, as will be discussed in section 5.7.1. For hydration times of 0 minutes up to 2 minutes hydration (excluding 0.5 minutes) the MTF curves are very similar, and at an MTF of 0.2 the resolution is comparable to the commercial plate at approximately $2.6 \mathrm{lp} / \mathrm{mm}$. For 5 minutes hydration the MTF has a Gaussian-like shape, which is due to the high opacity of the imaging plate. The pre-sintered imaging plate hydrated for 30 seconds shows a lower MTF than expected. This could be caused by poor optical quality of the imaging plate, or incorrect analysis of the grid data which determines the MTF. 


\subsection{Interpretation}

\subsubsection{Model for generation of the active PSL site}

The mechanisms for $\mathrm{CsBr}: \mathrm{Eu}^{2+}$ storage phosphor imaging plates are much more complicated than might expected for the apparent situation of a simple alkali halide with a rare earth dopant. Water plays a critical role in the formation of the PSL active site in the imaging plate. What follows is a model for the generation of the active PSL centres developed from the results given in this chapter.

During the milling process the powders are ground by grains being impacted between two milling balls, or between a milling ball and the container wall. The balls have a relatively high kinetic energy which is transferred to the powder in the form of heat. If both a $\mathrm{CsBr}$ and $\mathrm{EuBr}_{2}$ grain are crushed at the same time, thermomechanical transfer of some $\mathrm{Eu}^{2+}$ ions into the $\mathrm{CsBr}$ can occur, where it enters the $\mathrm{CsBr}$ lattice as a $\mathrm{Eu}^{2+}-\mathrm{Cs}_{\mathrm{v}}$ pair. These pairs are very mobile and can migrate through the lattice to form agglomerates [70]. The agglomerates are thus in equilibrium with a concentration of highly mobile isolated $\mathrm{Eu}^{2+}-\mathrm{Cs}_{\mathrm{v}}$ pairs, which can migrate to the surface to combine with surface water, following the reaction,

$$
\left[\mathrm{Eu}^{2+}+\mathrm{Cs}_{\mathrm{v}}\right]+\mathrm{H}_{2} \mathrm{O} \rightarrow\left[\mathrm{Eu}^{2+}+\mathrm{Cs}_{\mathrm{v}}+\mathrm{Cs}_{\mathrm{v}}\left(\mathrm{H}_{2} \mathrm{O}\right)\right]+\mathrm{Br}_{\mathrm{v}}
$$

or overall,

$$
\mathrm{EuBr}_{2}+\mathrm{H}_{2} \mathrm{O} \rightarrow\left[\mathrm{Eu}^{2+}+\mathrm{Cs}_{\mathrm{v}}+\mathrm{Cs}_{\mathrm{v}}\left(\mathrm{H}_{2} \mathrm{O}\right)\right]+2 \mathrm{Br}^{-}+\mathrm{Br}_{\mathrm{v}} .
$$

Here the reaction is viewed as taking place at an atomic step on the crystal surface, which facilitates the incorporation of a water molecule, the required generation of an additional caesium vacancy and the necessary associated 


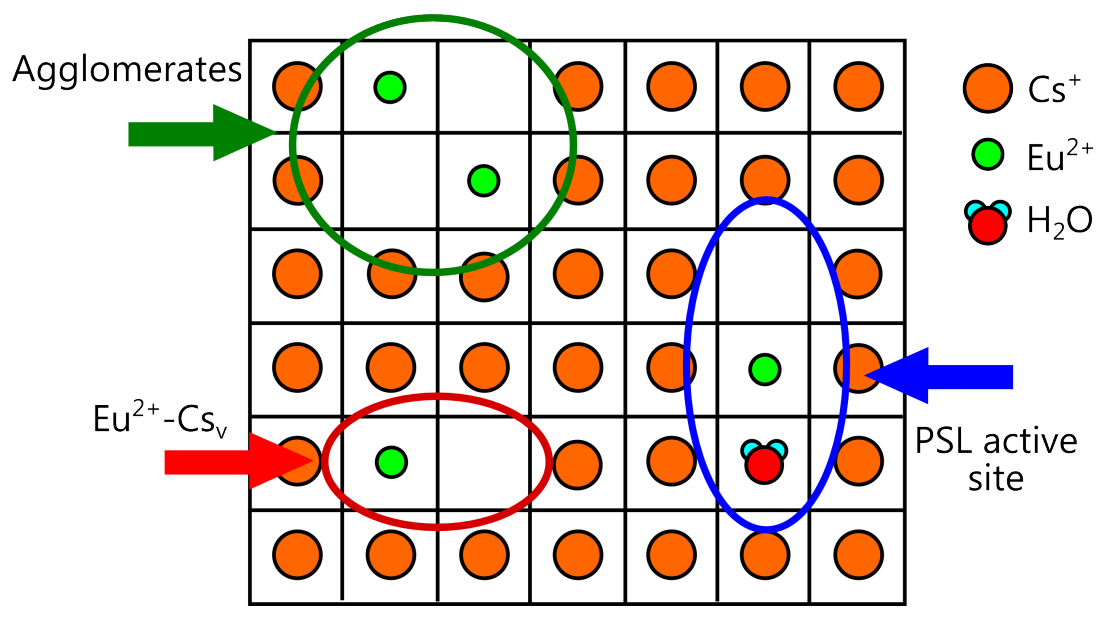

Figure 5.44: Representation of PSL active site, $\mathrm{Eu}^{2+}-\mathrm{Cs}_{\mathrm{v}}$ pair, and $\mathrm{Eu}^{2+}-\mathrm{Cs}_{\mathrm{v}}$ agglomerate. The squares represent the unit cell, with each corner containing a $\mathrm{Br}^{-}$ion.

bromine vacancy. Both defects are required for PSL. As the reaction proceeds, the aggregates are depleted. The surface water also facilitates recrystallisation, which buries the PSL active sites within the growing crystals.

In the as-made imaging plates the europium can be in three forms, as $\mathrm{EuBr}_{2}$ (or $\left.\mathrm{EuBr}_{2} .1 \mathrm{H}_{2} \mathrm{O}\right)$, combined with water in the PSL active site, and/or as $\mathrm{Eu}^{2+}-\mathrm{Cs}_{\mathrm{v}}$ agglomerates. The structure of the PSL active site, $\mathrm{Eu}^{2+}-\mathrm{Cs}_{\mathrm{v}}$ pairs and agglomerates are shown schematically in figure 5.44. These three forms are evident in the photoluminescence emission spectra, for example figure 5.8(a), where peaks at $\sim 420,440$ and $485 \mathrm{~nm}$ are the three above forms respectively. If the starting materials are particularly anhydrous a two peak spectrum corresponding to $\mathrm{EuBr}_{2}$ and $\mathrm{Eu}^{2+}-\mathrm{Cs}_{\mathrm{v}}$ agglomerates may be seen (figure 5.15(a)).

When post hydrating the imaging plate, the water is first taken up as a thin film over the disc surfaces. This water generates a recrystallisation causing the water to progressively penetrate macroscopically though the plate (figure 5.6), and coating any dry pore surfaces with a film of water. The pancaked crystals reduce strain-energy by regrowth based on the high mobility of $\mathrm{Cs}$ and $\mathrm{Br}$ in the surface film; crystallite growth from aqueous solution is very fast [66]. This can also be applied to the imaging plates in moist air. The crystallite growth will occur via atomic steps on the surface generated for example by screw 
dislocations in the strained crystal. Europium present as $\mathrm{EuBr}_{2}$ can dissolve in the surface water, resulting in a distribution of $\mathrm{Eu}^{2+}$ ions over the surface of the $\mathrm{CsBr}$, and can directly interact with the water molecules creating the PSL active site at atomic steps on the crystal surface. Similarly, any $\mathrm{Eu}^{2+}-\mathrm{Cs}_{\mathrm{v}}$ centres already present in the $\mathrm{CsBr}$ can migrate to the reconstructing surface and also generate the PSL active site. Any PSL active site created on a surface step will be buried within the crystal by successive regrowth layers. The low thermal roughening temperature of $\mathrm{CsBr}$ in an aqueous solution [65] means that any crystallites which are formed are rounded in nature rather than as sharp crystal faces. The formation of the PSL active site corresponds to a decrease in the 420 and $485 \mathrm{~nm}$ peaks in the PL spectra ( $\mathrm{EuBr}_{2}$ and aggregates), and a growth in the $440 \mathrm{~nm}$ peak (PSL active site).

When hydrating as a powder before pressing, the water may be first taken up preferentially by the $\mathrm{EuBr}_{2}$ which recrystallises into $\mathrm{EuBr}_{2} \cdot 1 \mathrm{H}_{2} \mathrm{O}$, as evident by the increasing $\mathrm{EuBr}_{2} \cdot 1 \mathrm{H}_{2} \mathrm{O}$ peaks in the IR spectra (figure 5.14). The PL emission peak associated with the agglomerates decreases much quicker than the $\mathrm{EuBr}_{2}$ peak (figure 5.15), which suggests that the PSL active sites are first generated by the agglomerates decomposing. This again is associated with an increase in the CE.

Annealing the imaging plates up to temperatures of $100{ }^{\circ} \mathrm{C}$ can cause any water in the $\mathrm{CsBr}$ pores to be released and absorbed by the $\mathrm{EuBr}_{2}$ to form $\mathrm{EuBr}_{2} \cdot 1 \mathrm{H}_{2} \mathrm{O}$. This is evident from the increasing $\mathrm{EuBr}_{2} \cdot 1 \mathrm{H}_{2} \mathrm{O}$ peaks in the IR spectra (figure 5.24). These peaks are strongest at $50{ }^{\circ} \mathrm{C}$, and in the UV-Vis extinction spectra suggest that the $\mathrm{EuBr}_{2} \cdot 1 \mathrm{H}_{2} \mathrm{O}$ correlates with a peak at approximately $280 \mathrm{~nm}$. Unfortunately, PL spectra were not recorded for these samples, but both the UV-Vis extinction (figure 5.23) and EPR spectra (figure 5.26) show the changing stages of the europium site structure. Annealing at temperatures of $150-250$ ${ }^{\circ} \mathrm{C}$ show EPR spectra which are equivalent to those reported for the active PSL site in literature [36]. This is correlated with an increase in the CE between 
these temperatures. The UV-Vis extinction spectra (figure 5.23) at these temperatures show two peaks at 260 and $340 \mathrm{~nm}$, which correspond to the $\mathrm{e}_{\mathrm{g}}$ and $\mathrm{t}_{2 \mathrm{~g}}$ transitions of europium in the PSL active site. The latter should be split by the axial crystal field, but this is not resolved. Increasing the annealing temperature above $250{ }^{\circ} \mathrm{C}$ leads to a decrease in the conversion efficiency, which is due to the water molecules being expelled from the PSL active site. This correlates with a change in the EPR spectrum, which becomes broader and is associated with $\mathrm{Eu}^{2+}$ ions in a weak crystal field, possibly isolated $\mathrm{Eu}^{2+}$ ions.

Annealing as a powder is less successful than annealing the pressed imaging plate. Initially the PL spectra in figure 5.31 shows europium in the PSL active site which is associated with a moderate conversion efficiency. As the temperature is increased to $50{ }^{\circ} \mathrm{C}$ the $\mathrm{EuBr}_{2}$ hydrates to produce $\mathrm{EuBr}_{2} \cdot 1 \mathrm{H}_{2} \mathrm{O}$, along with some europium in the PSL active site. This is seen as an increase in the infrared peaks associated with $\mathrm{EuBr}_{2} \cdot 1 \mathrm{H}_{2} \mathrm{O}$ in figure 5.30, as well as two peaks in the PL spectrum in figure 5.31. Increasing the temperature further drives off water (seen as a decrease in water associated peaks in figure 5.30) and so does not allow the water to interact with the $\mathrm{Eu}^{2+}-\mathrm{Cs}_{\mathrm{v}}$ agglomerates to form the PSL active site. Instead the europium exists in the $\mathrm{EuBr}_{2}$ and $\mathrm{Eu}^{2+}-\mathrm{Cs}_{\mathrm{v}}$ forms.

Pre-sintering the powder before pressing and hydrating leads to the incorporation of the $\mathrm{EuBr}_{2}$ into the CsBr lattice to give the PSL active site and $\mathrm{Eu}^{2+}-\mathrm{Cs}_{\mathrm{V}}$ agglomerates. The formation of the active site before hydration is surprising but could be caused during the transfer of the sintered powder from the furnace to dry box in room air. Any hydration is directly converted to the PSL active site by dissociating the agglomerates, which leads to an increases $\mathrm{CE}$, over and above that found for the non-sintered imaging plates.

The above model fits well with the data presented in this chapter. However, further investigations need to be undertaken in order to verify that the model is indeed correct. 


\subsubsection{Spatial resolution}

The shape of the modulation transfer function for the pressed $\mathrm{CsBr}: \mathrm{Eu}^{2+}$ imaging plate is significantly different from the commercial imaging plate. At low spatial frequencies the pressed imaging plates show a much narrower MTF, which flattens out to a relatively constant value at higher spatial frequencies. In contrast, the MTF for the commercial imaging plate has a Gaussian-type shape, where the MTF is broader at low spatial frequencies and decreases to zero at higher spatial frequencies. The higher MTF values for the pressed imaging plates at high frequencies compared to the commercial needle imaging plates is due to their semi-transparent nature. This higher value of the MTF at high frequencies, evident qualitatively in figure 5.11, is a crucial advantage of the materials developed here.

At low frequencies the difference in width of the MTFs can be explained by the differing paths the emission light takes within the imaging plate. For the purpose of this discussion, the PSL emission in a transparent $\mathrm{CsBr}$ imaging plate is assumed to originate from an infinitesimally thin line of X-ray irradiation - the equivalent of a point source. For a perfectly transparent imaging plate the light travels without scattering until it reaches a surface, where it is either totally internally reflected or exits the imaging plate (see figure 5.45(a)). This is

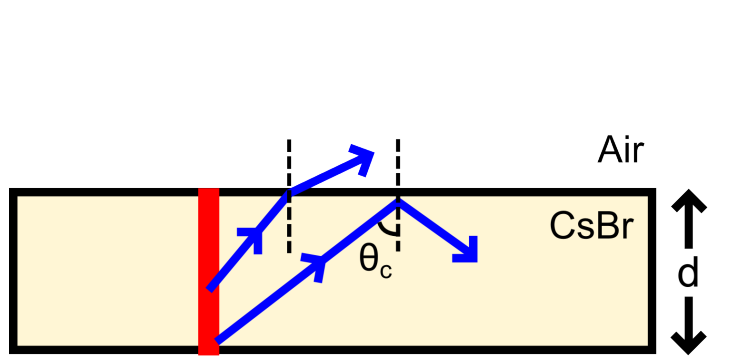

(a)

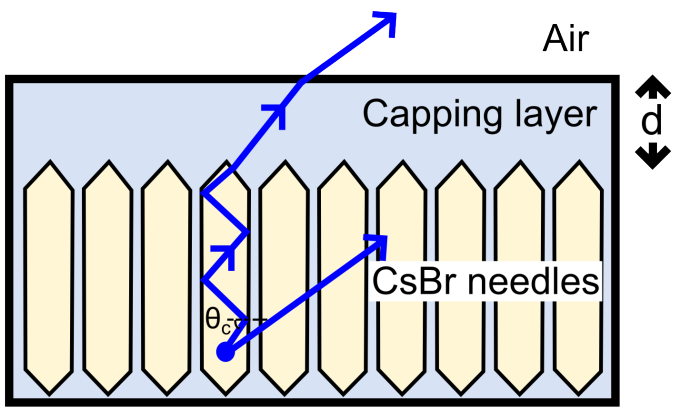

(b)

Figure 5.45: Light scattering schematic for (a) transparent and (b) needle imaging plate. 
defined by the critical angle at the $\mathrm{CsBr}$, air interface,

$$
\theta_{c}=\sin ^{-1}\left(\frac{n_{2}}{n_{1}}\right) \approx 36^{\circ}
$$

where $n_{1}$ and $n_{2}$ are the refractive indices for $\mathrm{CsBr}$ and air respectively. At the surface of the imaging plate the light appears as a strip (if assuming a point source a circle would be seen) with width,

$$
w=2 d \tan \theta_{c}
$$

where $d$ is the thickness of the imaging plate. Without calculating the specific intensity distribution, the intensity variation across the strip (in one dimension) can be approximated by a cosine pulse,

$$
I(x)=I_{0} \cos \left(\frac{\pi x}{2 d \tan \theta_{c}}\right) \operatorname{rect}\left(\frac{x}{2 d \tan \theta_{c}}\right)
$$

where $x$ is the distance from the central maximum, $I_{0}$ is the intensity at $x=0$, and rect is the top-hat function of width $2 d \tan \theta_{c}$. Equation 5.6 defines the line spread function of the image which can be converted into the MTF by taking the Fourier transform. The Fourier transform of equation 5.6 is well known [71] and is given by,

$$
M T F=\frac{I_{0} \pi}{d \tan \theta_{c}} \frac{\cos \left(2 \pi \mu d \tan \theta_{c}\right)}{\left(\frac{\pi}{2 d \tan \theta_{c}}\right)^{2}-(2 \pi \mu)^{2}}
$$

where $\mu$ is the spatial frequency in line pairs $/ \mathrm{mm}$. This function is plotted in figure 5.46 for a $300 \mu \mathrm{m}$ thick CsBr imaging plate and falls to half its initial value at $1.9 \mathrm{lp} / \mathrm{mm}$.

For a needle imaging plate, the same infinitesimally thin line of X-rays will activate a single needle (or strictly a row of needles). Any light emitted where $\theta>\theta_{c}$ will be guided up the needle to the surface (figure $5.45(\mathrm{~b})$ ), with the rest travelling though to the next needle. When the light reaches the surface it encounters a capping layer designed to protect the needles from damage and 


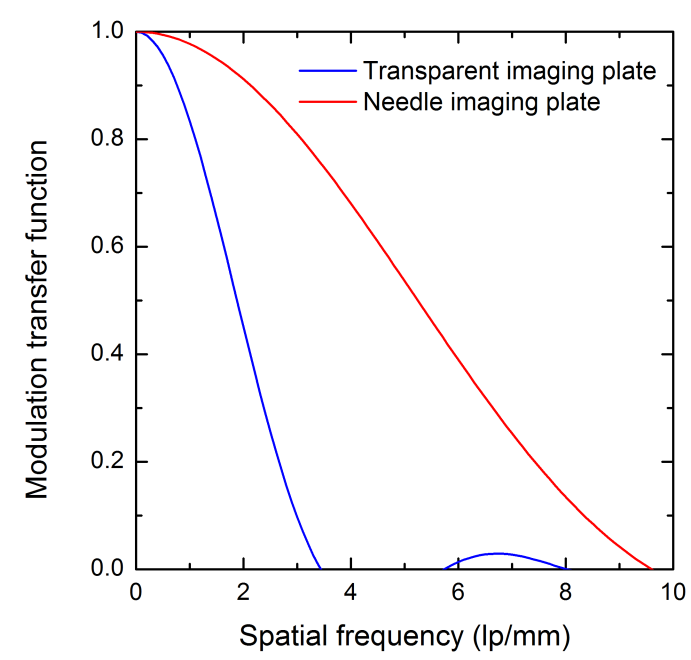

Figure 5.46: Estimated MTF at low frequencies for transparent and needle imaging plates.

moisture. Typically this layer consists of parylene with a thickness between $10-100 \mu \mathrm{m}$ [72] which has a refractive index of $1.64-1.67$ depending on actual composition. The MTF can again be determined using equation 5.7, with the parameters $d$ and $\theta_{c}$ now from the capping layer. This is shown in figure 5.46, assuming a capping thickness of $100 \mu \mathrm{m}$ and $\theta_{c}=36^{\circ}$. The curve falls to a value of 0.5 at approximately $5.3 \mathrm{lp} / \mathrm{mm}$. As can be seen the MTF at low spatial frequencies is much higher for the commercial needle imaging plate than for a transparent imaging plate. This gives a semi-quantitative explanation of the difference in spatial resolution at low frequencies. The full picture is complicated by internal and surface scattering for both cases.

\subsection{Comparison of processes}

A comparison of the best results for the preparation processes are shown in table 5.3. Best in this case is defined as the production process which gives the imaging plate with the highest conversion efficiency. 


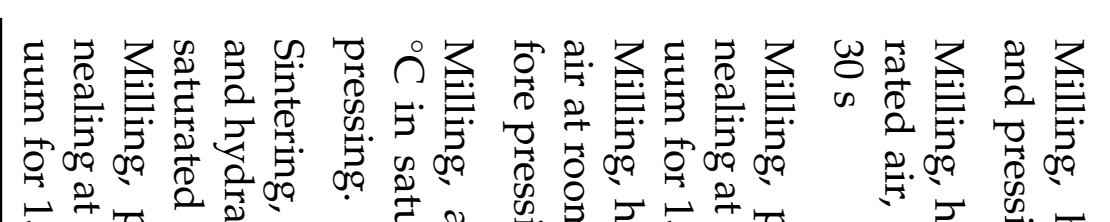

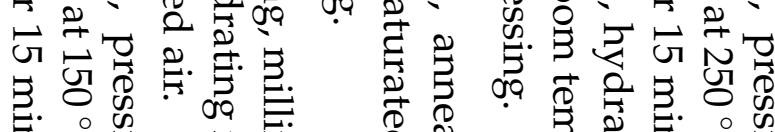

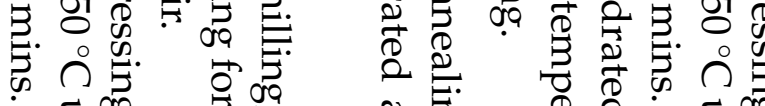

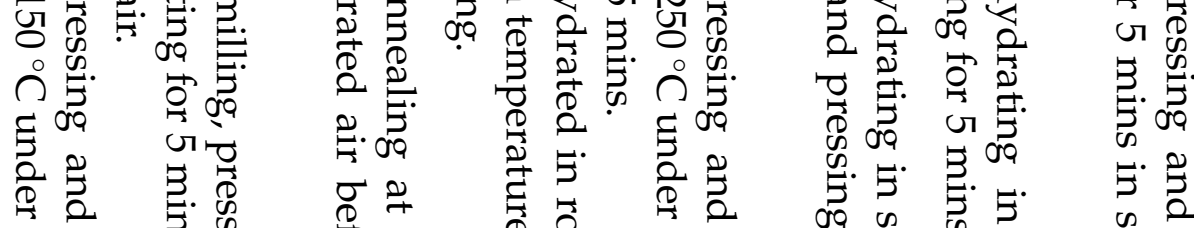

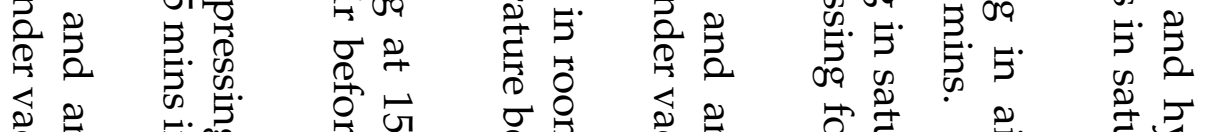

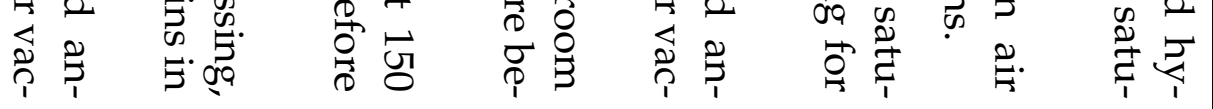

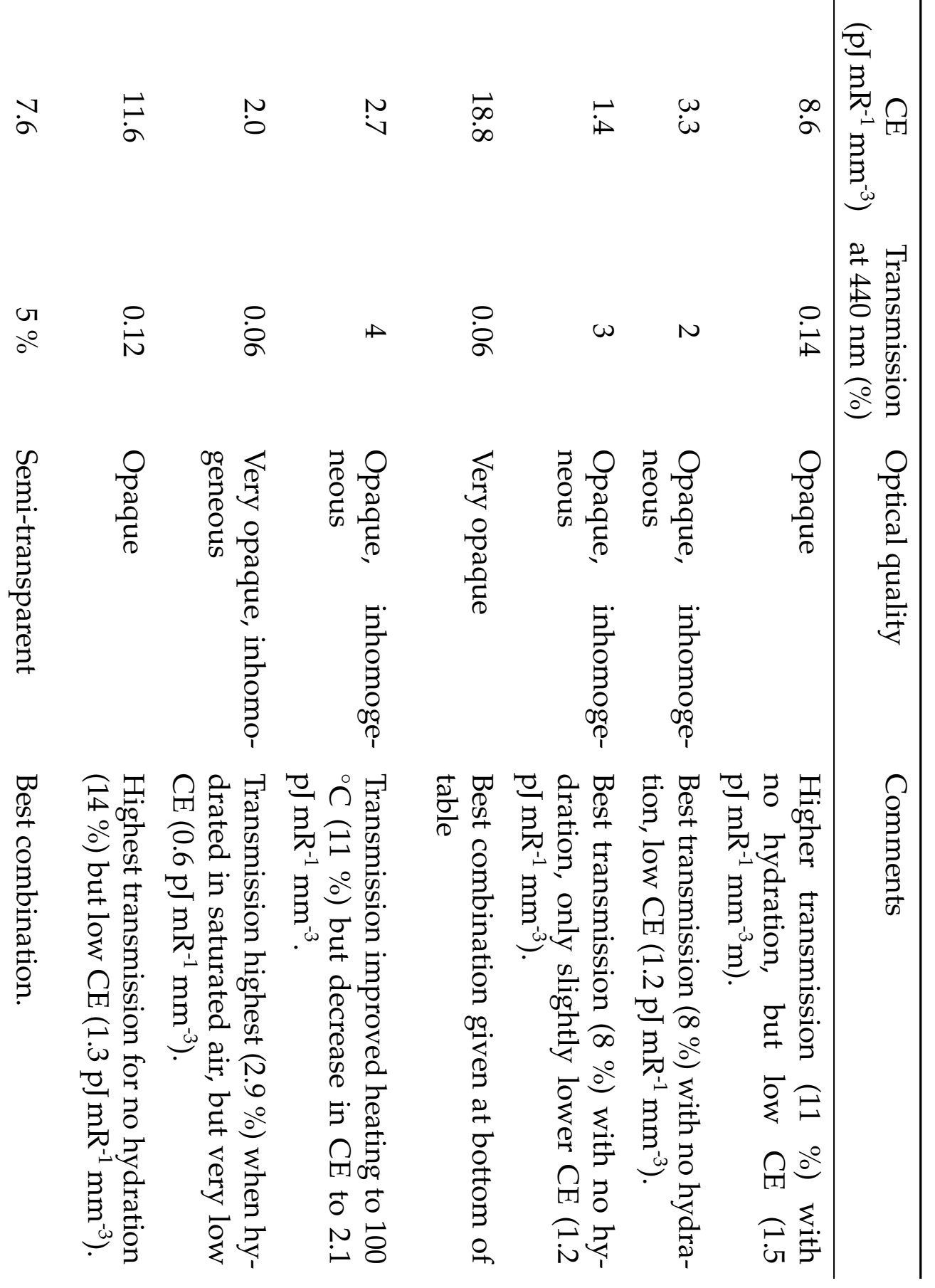

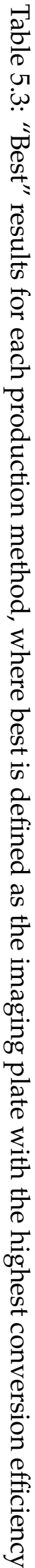




\subsection{Examples of images}

Representative X-ray images taken using various hydrated pressed imaging plates are given in figure 5.47

\subsection{Conclusions}

- There is no evidence from the infrared spectra for the formation of $\mathrm{OH}^{-}$ ions during the hydration process.

- Peaks in the range of $2900 \mathrm{~cm}^{-1}$ in the IR spectra are an artefact due to hydrocarbon contamination.

- A set of four peaks in the infrared spectra at $3488,3440,1604$ and $584 \mathrm{~cm}^{-1}$ are due to hydrated $\mathrm{EuBr}_{2}$ as $\mathrm{EuBr}_{2} \cdot 1 \mathrm{H}_{2} \mathrm{O}$. Their presence in the IR spectra suggest that the $\mathrm{EuBr}_{2}$ has not been completely incorporated into the $\mathrm{CsBr}$ which is undesirable for optimal PSL intensity. This is an indicator of an non-optimal process.

- The prime indicator of a successful conversion process is the PL line at 440 $\mathrm{nm}$. The excitation spectrum for this line is comprised of an unresolved $t_{2 g}$ band at $345 \mathrm{~nm}$ and an $e_{\mathrm{g}}$ band at $270 \mathrm{~nm}$.

- A line in the infrared at approximately $3200 \mathrm{~cm}^{-1}$ also appears to be a prerequisite for a substantial PSL. The intensity of this line does not directly correlate with the $\mathrm{CE}$, so it is unclear whether this is the $\nu_{1}$ or $\nu_{3}$ vibration of the $\mathrm{H}_{2} \mathrm{O}$ molecule in the active PSL site. It should be noted that the CE also depends upon the F-centre concentration as well as on the active hole site.

- The highest conversion efficiency $\left(18.8 \mathrm{pJ} \mathrm{mR}^{-1} \mathrm{~mm}^{-3}\right)$ is obtained from an imaging plate which initially contains a relatively high water content and is subsequently annealed at $250{ }^{\circ} \mathrm{C}$ under vacuum. 


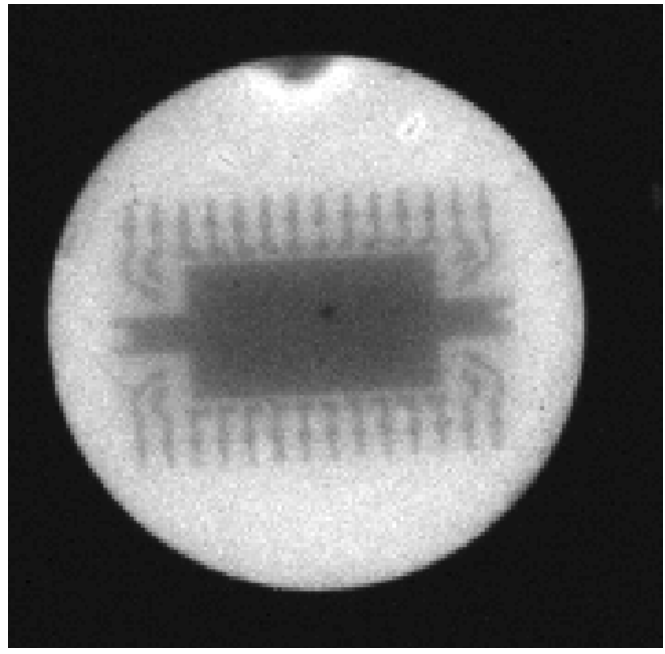

(a)

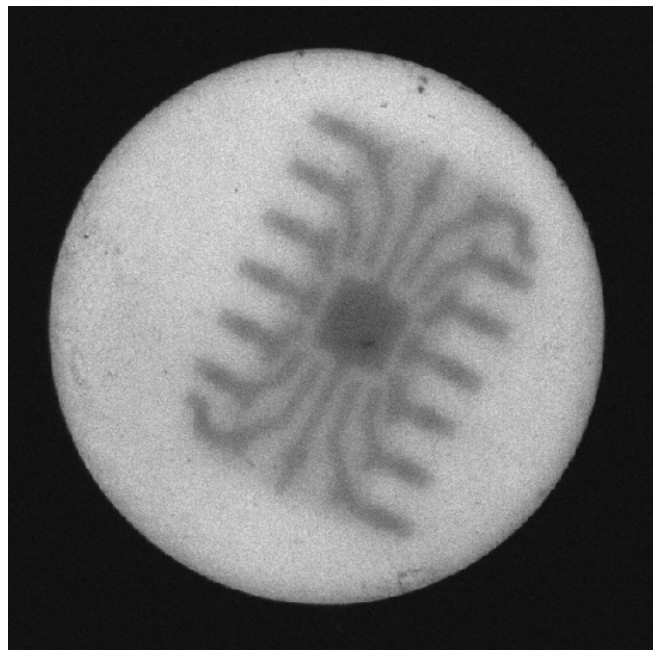

(c)

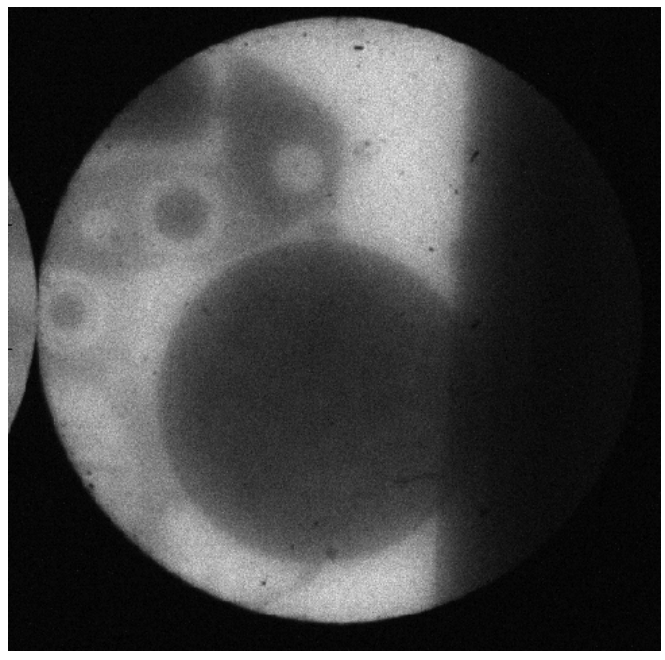

(e)

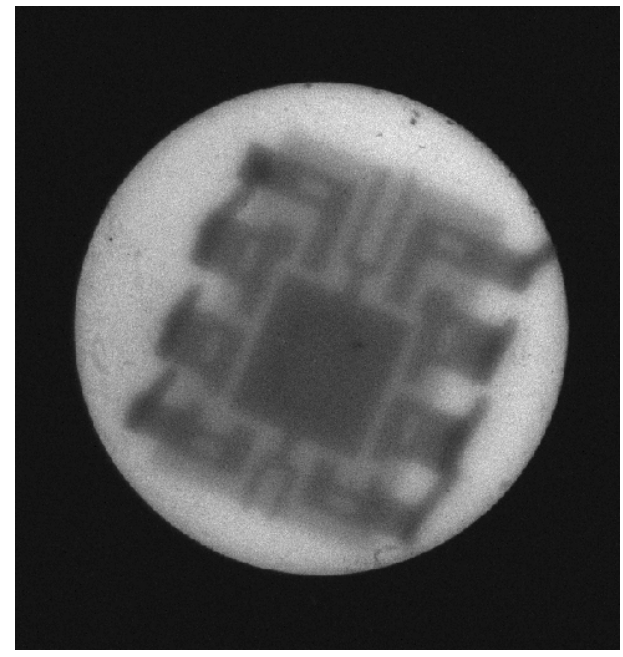

(b)

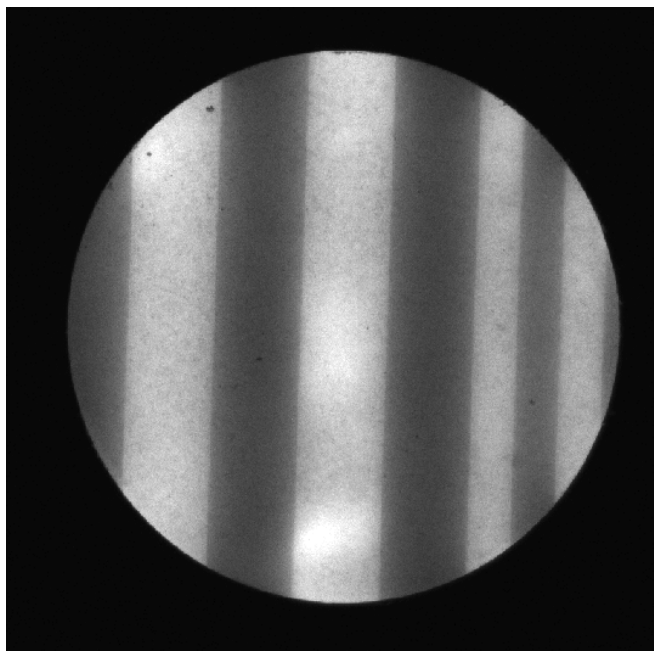

(d)

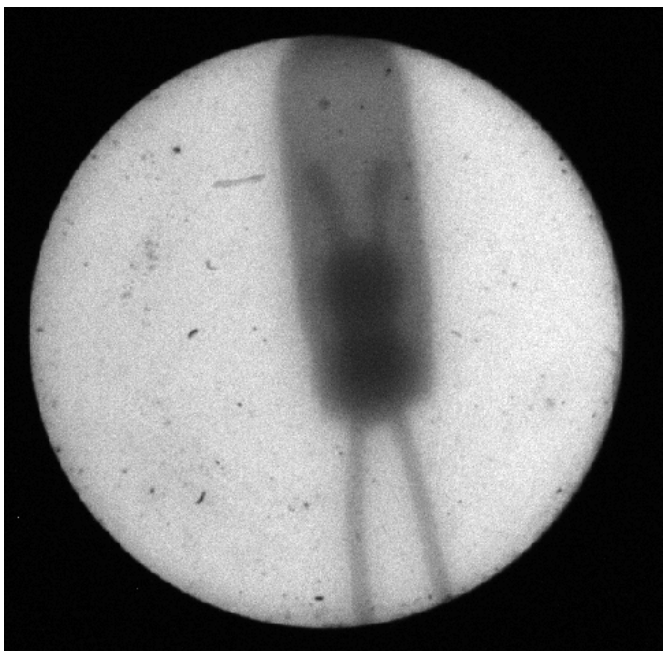

(f)

Figure 5.47: X-ray images taken with (13 $\mathrm{mm}$ diameter) hydrated pressed CsBr:Eu ${ }^{2+}$ imaging plates (Method 1 or 5). (a) - (c) Integrated circuit chips, (d) lead grid (low spatial frequencies), (e) part of a watch and (f) small torch light bulb. 
- Imaging plates which have been pre-sintered as a powder show higher conversion efficiencies $\left(11.6 \mathrm{pJ} \mathrm{mR}^{-1} \mathrm{~mm}^{-3}\right)$ after hydration in saturated air, as opposed to those not sintered $\left(\mathrm{CE}=8.6 \mathrm{pJ} \mathrm{mR}^{-1} \mathrm{~mm}^{-3}\right)$.

- The opacity of the sample is controlled by the processes of crystal regrowth and the uptake of water by the pores. Both change the total contact area between the $\mathrm{CsBr}$ crystallites, which decreases the transparency, and the area of $\mathrm{CsBr}$ to air (or water) interface which leads to greater optical scattering.

- Methods 2 and 4 lead to optically inhomogeneous materials which are therefore unsuited for imaging plates. In addition the $\mathrm{CE}$ is inferior to that in methods 1, 3 and 5, which give optically homogeneous materials.

- Methods 1, 3 and 5 have resulted in transparent or semi-transparent homogeneous storage phosphors with a high frequency MTF and a CE which is superior to the commercial needle imaging plates.

- The optimal preparation process is to anneal the pressed imaging plate at $150{ }^{\circ} \mathrm{C}$ (to preserve some transparency) either in room or saturated air, depending on the water content required. Ideally, the required amount of water should be added to the starting powders (either $\mathrm{CsBr}$ or $\mathrm{EuBr}_{2}$ ) before milling and pressing the imaging plates, and then annealing at 150 ${ }^{\circ} \mathrm{C}$ under vacuum. 



\section{Chapter 6}

\section{Dual-energy imaging with stratified CsBr imaging plates}

Conventional X-ray radiography provides a representation of an object in terms of the product of attenuation coefficient and material thickness. The attenuation of X-rays through a material is given by,

$$
I=I_{0} \exp (-\mu(x, y) z)
$$

with,

$$
\mu(x, y) z=\sum_{i}\left(\frac{\mu_{i}}{\rho_{i}}\right) \rho_{i}(x, y) z_{i},
$$

where $\rho$ is the material density, $\mu$ the attenuation coefficient, $z$ the thickness and the sum is over the elements making up the material. $\left(\mu_{i} / \rho_{i}\right)$ is the mass attenuation coefficient of element $i$. The information given in such an X-ray image is not sufficient to chemically characterise the object which is being imaged, since many combinations of materials and their thickness can give the same net attenuation. To extract some compositional information, a dual-energy technique can be used which involves recording two images at different energies. This technique takes advantage of the fact that the attenuation of X-rays in matter (at energies less than $1 \mathrm{MeV}$ ) is due to a combination of the photo-electric effect 
and Compton scattering, which have different dependencies on atomic number and energy. The attenuation coefficient, $\mu$, for a particular element above the $\mathrm{K}$ edge can be written in terms of the component photoelectric $\left(\mu_{\mathrm{PE}}\right)$ and Compton scattering $\left(\mu_{\mathrm{CS}}\right)$ attenuation coefficients, that is,

$$
\mu=\mu_{\mathrm{PE}}+\mu_{\mathrm{CS}}
$$

These two interactions and their contribution to the attenuation are dependent on the atomic number and X-ray energy in a well known way. Therefore, using the information from the two separate energy images, the attenuation can be split into its two contributions. This in turn can be used to produce material specific images, or determine relative thicknesses of components, or produce images with an improved contrast.

An example of an application using this technique is dual-energy X-ray absorptiometry (DXA or DEXA) which is a means of measuring bone mineral density. Another medical application is the generation of images which have bone or tissue subtracted images, such as those shown in figure 6.1(a) which are used as an improved diagnostic tool. Baggage scanners at airport security also use dual-energy imaging to determine object composition and identify potentially hazardous materials. An example image is shown in figure 6.1(b), where the objects are colour coded to represent organic or inorganic materials, or metals. A novel application is the use of a dual-energy X-ray scanner in meat distribution facilities to determine the ratio of lean meat to fat in packaged (boxed) meat products [75].

There are two standard methods for generating the two images required for dual-energy imaging. The first is the dual exposure technique (two-shot), where two separate acquisitions are performed with the X-ray energy being switched between each exposure, usually by changing the X-ray voltage and using filters. This method has very good energy separation but requires a static object for best results to avoid motional blurring. The second method is the single exposure 


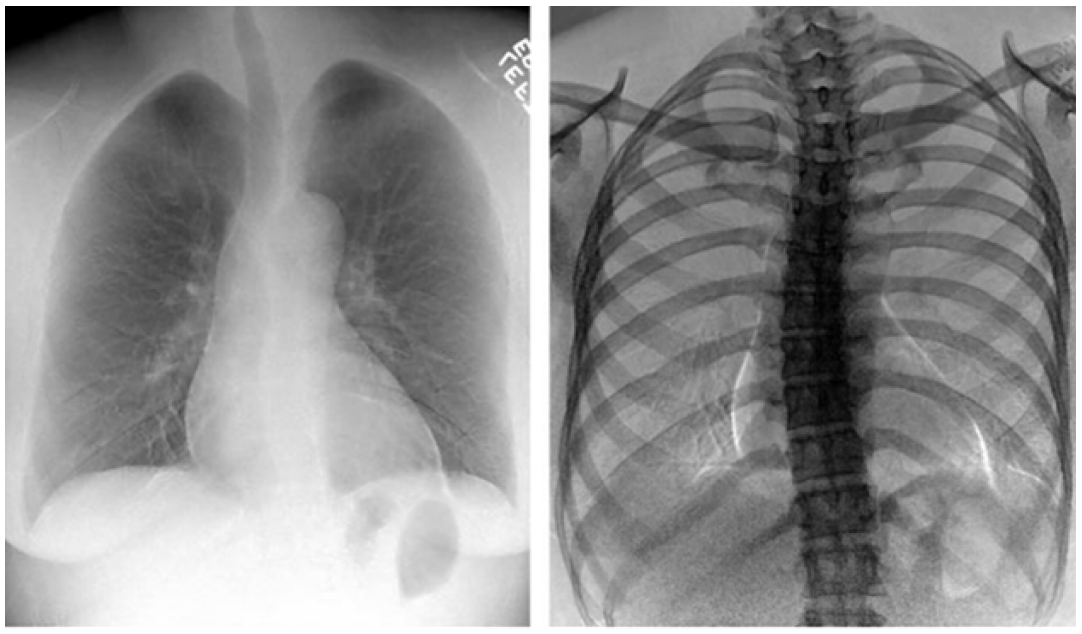

(a)

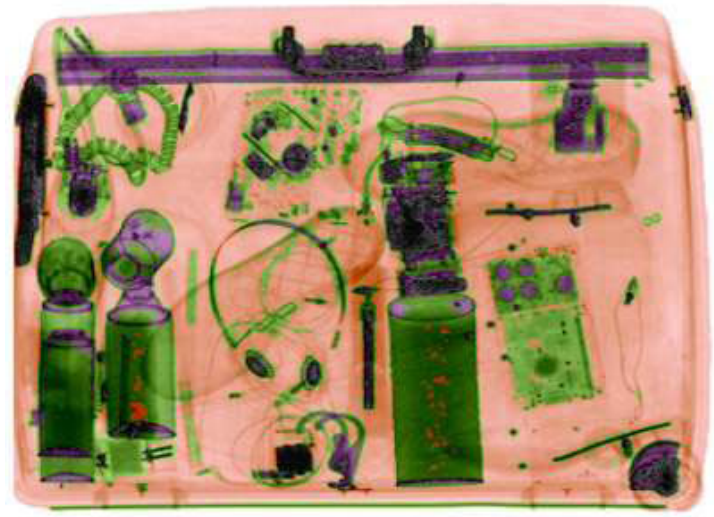

(b)

Figure 6.1: Dual-energy imaging examples, (a) bone (left) and tissue (right) subtracted images of a chest [73], and (b) airport baggage scan image [74].

technique (one-shot), which is the method implemented in this thesis. This method uses a poly-energetic X-ray source with a sandwich detector consisting of two imaging layers separated by a filter to create the two images. The top layer and the filter absorb most of the low energy photons, and as a consequence the bottom layer detects mostly high energy photons. The intermediate filter is used to absorb X-rays in order to increase the energy separation. As the images are obtained simultaneously, moving objects can be imaged without motional blurring. However, the energy separation is usually poor and in the case of imaging plates as the detector layers, registration of the two images (which need to be read out with separate geometries) can be difficult.

A solution to the registration of images problem, which is proposed and investi- 
gated here, is to use a two layer imaging plate detector, where each layer has a different dopant with a distinct colour emission. The two energy images can then be separated on the basis of emission colour during the read out stage using the digital colour camera read-out system described in chapter 4 . In this chapter the standard algorithms for decomposing dual energy images will first be discussed. Secondly, material syntheses aimed at developing CsBr imaging plates with different dopants for their use in the dual-energy imaging plate system outlined above will be presented. Finally, their performance and dual-energy imaging capabilities are discussed.

\subsection{Dual-energy algorithms}

The original dual-energy algorithm by Alvarez and Marcovski [76] is based on the principle that the attenuation coefficient for a material can be written as the linear combination of two functions,

$$
\mu(\mathrm{E})=a_{1} \frac{1}{E^{3.2}}+a_{2} f_{\mathrm{KN}}(E)
$$

where the first term represents the photoelectric interaction and the second represents Compton scattering of X-rays of energy E. $f_{\mathrm{KN}}(E)$ is the KleinNishina formula,

$$
f_{\mathrm{KN}}(E)=\frac{1+\gamma}{\gamma^{2}}\left[\frac{2(1+\gamma)}{1+2 \gamma}-\frac{1}{\gamma} \ln (1+2 \gamma)\right]+\frac{1}{2 \gamma} \ln (1+2 \gamma)-\frac{(1+3 \gamma)}{(1+2 \gamma)^{2}}
$$

where $\gamma=E / m c^{2}$. This is strictly only valid when the X-ray energy is below the pair production region $(<1 \mathrm{MeV})$, and the materials being imaged have no K-edges in the region of interest. The coefficients $a_{1}$, and $a_{2}$ are given by,

$$
\begin{aligned}
& a_{1}=k_{1} \frac{\rho}{A} Z^{3.8}, \\
& a_{2}=k_{2} \frac{\rho}{A} Z
\end{aligned}
$$


where the $k^{\prime}$ s are constants, $Z$ the atomic number, $A$ the atomic mass number and $\rho$ is the mass density.

Equation 6.4 expresses the attenuation coefficient, viewed as a function of energy, as a linear sum of two basis functions, namely the photoelectric and Compton components. The coefficients (equations 6.6 and 6.7) are set by the particular chemical element. It follows from this that the attenuation coefficient as a function of energy of any material $\xi$ can be expressed as a linear combination of two arbitrary basis materials $\alpha$ and $\beta$ [77],

$$
\frac{\mu_{\xi}(E)}{\rho_{\xi}}=a_{\alpha} \frac{\mu_{\alpha}(E)}{\rho_{\alpha}}+a_{\beta} \frac{\mu_{\beta}(E)}{\rho_{\beta}}
$$

To apply this concept to an image, a logarithmic transmission is used, which is defined as,

$$
M=\log \left(\frac{I_{0}}{I}\right)=\mu(E) t
$$

where $t$ is the material thickness. For the basis materials this is found by multiplying equation 6.8 by the density and thickness of material $\xi[78,79]$,

$$
\begin{aligned}
M & =\mu_{\xi}(E) t_{\xi}, \\
& =\left(\frac{a_{\alpha} \rho_{\xi} t_{\xi}}{\rho_{\alpha}}\right) \mu_{\alpha}(E)+\left(\frac{a_{\beta} \rho_{\xi} t_{\xi}}{\rho_{\beta}}\right) \mu_{\beta}(E), \\
& =A_{\alpha} \mu_{\alpha}(E)+A_{\beta} \mu_{\beta}(E),
\end{aligned}
$$

where $A_{\alpha}$ and $A_{\beta}$ are equivalent thicknesses, independent of energy. In dualenergy imaging two logarithmic transmissions are defined,

$$
\begin{gathered}
M_{h}=A_{\alpha} \mu_{\alpha}\left(E_{h}\right)+A_{\beta} \mu_{\beta}\left(E_{h}\right), \\
M_{l}=A_{\alpha} \mu_{\alpha}\left(E_{l}\right)+A_{\beta} \mu_{\beta}\left(E_{l}\right),
\end{gathered}
$$




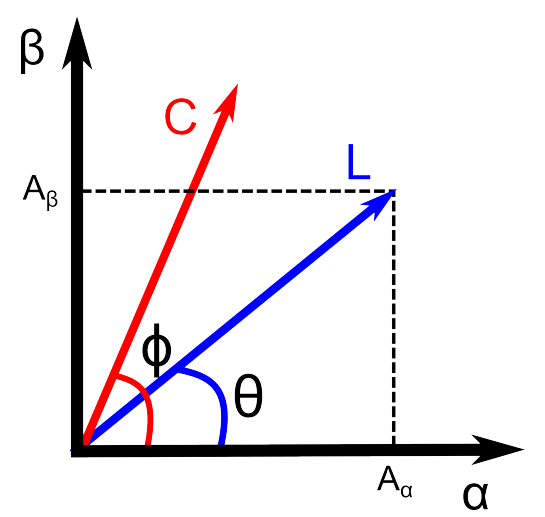

Figure 6.2: The basis material plane $\alpha, \beta$ where $L$ is a vector representing material $\xi$ with equivalent thicknesses $A_{\alpha}$ and $A_{\beta}$. $C$ is the basis projection image as defined in the text [77].

for the high and low energy beams respectively. Equations 6.11 and 6.12 can be solved for the equivalent thicknesses, as follows,

$$
\begin{aligned}
A_{\alpha} & =\frac{M_{h} \mu_{\beta}\left(E_{l}\right)-M_{l} \mu_{\beta}\left(E_{h}\right)}{\mu_{\alpha}\left(E_{h}\right) \mu_{\beta}\left(E_{l}\right)-\mu_{\beta}\left(E_{h}\right) \mu_{\alpha}\left(E_{l}\right)}, \\
A_{\beta} & =\frac{M_{l} \mu_{\alpha}\left(E_{h}\right)-M_{h} \mu_{\alpha}\left(E_{l}\right)}{\mu_{\alpha}\left(E_{h}\right) \mu_{\beta}\left(E_{l}\right)-\mu_{\beta}\left(E_{h}\right) \mu_{\alpha}\left(E_{l}\right)} .
\end{aligned}
$$

The equivalent thicknesses $A_{\alpha}$ and $A_{\beta}$ represent the thickness of base materials, $\alpha$ and $\beta$, which together give the same attenuation as material $\xi$. Typical basis materials for medical imaging are aluminium and PMMA [77] as they encompass the full range of atomic numbers for diagnostic radiology.

The logarithmic transmission, $M$, of any material can be represented by a vector in a two dimensional basis plane, as shown in figure 6.2. The axes represent the basis materials $\alpha$ and $\beta$, and $A_{\alpha}$ and $A_{\beta}$ are the projections of the vector on the axes. The vector has length $L=\sqrt{A_{\alpha}^{2}+A_{\beta}^{2}}$ which is proportional to the thickness of material $\xi$, and makes a characteristic angle $\theta=\tan ^{-1}\left(A_{\beta} / A_{\alpha}\right)$ with the $\alpha$ axis. For two fixed basis materials, each represented by a vector along the $\alpha, \beta$ axes, any other element can be represented by a fixed direction vector in the $\alpha, \beta$ plane. Conversely, any arbitrary image can be decomposed into $A_{\alpha}(x, y)$ and $A_{\beta}(x, y)$ component images.

A "basis projection image" can be formed by projecting the $\left(A_{\alpha}, A_{\beta}\right)$ vector onto 
a unit vector aligned at an angle $\phi$, pointing away from the origin (figure 6.2). The projection of $\left(A_{\alpha}, A_{\beta}\right)$ on that unit vector is,

$$
C=A_{\alpha} \cos \phi+A_{\beta} \sin \phi
$$

For a homogeneous unknown material, the effective atomic number can be found by forming a number of basis projection images, and varying the angle $\phi$ until the object disappears $(C=0)$. The vector defined by $\phi$ is then perpendicular to the unit vector at the characteristic angle $\theta$ for that material. The atomic number $(Z)$ of the homogeneous material can then be found using the characteristic angle using,

$$
Z^{3.8}=\frac{\rho_{\beta} N_{g \beta} Z_{\beta}^{3.8} \tan \theta+\rho_{\alpha} N_{g \alpha} Z_{\alpha}^{3.8}}{\rho_{\beta} N_{g \beta} \tan \theta+\rho_{\alpha} N_{g \alpha}}
$$

where $N_{g}=\frac{Z}{A} N_{A}, A$ the atomic mass number and $N_{A}$ is Avogadro's number [77].

For layered materials the image vectors add. Figure 6.3 shows the image vectors for the case of $X$-rays travelling through water and then iodine, and for water and then bone. In both cases the sums are identical and the materials cannot be distinguished. Real objects however have a distinct shape, and as the materials are assumed to be displaced, each material can be separated geometrically. This is done by varying the projection angle until the known feature disappears. At this angle the projection angle is perpendicular to the materials characteristic angle. This allows for identification of unknown materials and forces the cancellation of an unwanted layer, enhancing the contrast of other layers.

When one material displaces another material, the contrast between two materials can be forced to vanish while still contrasting with a third material. The side-on geometry of this situation is shown in figure 6.4(a), which shows two materials $\psi$ and $\kappa$ displacing material $\xi$. X-rays passing through this material have three path options, labelled by the logarithmic transmissions $\mathrm{M}_{\xi}, \mathrm{M}_{\psi}$ and 


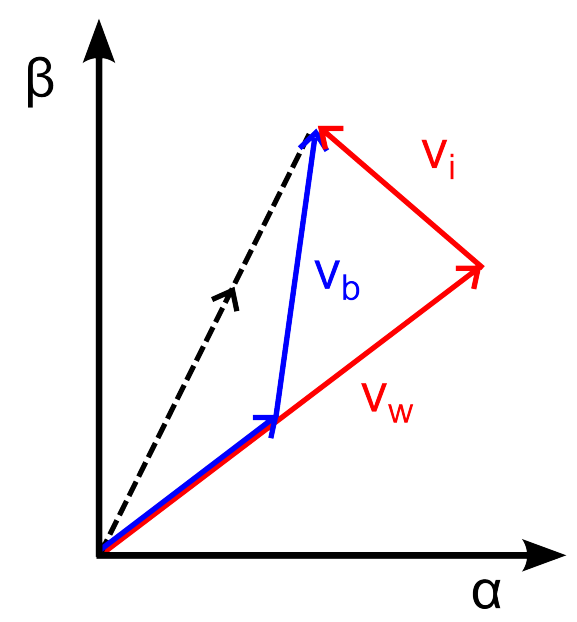

Figure 6.3: Vector addition of intervening materials for the cases of intervening iodine and water (red lines) and bone and iodine (blue lines), with different water thicknesses, where the sums are identical [77].

$\mathrm{M}_{\kappa}$, and given by,

$$
\begin{aligned}
& M_{\xi}=\mu_{\xi}(E) L, \\
& M_{\psi}=\mu_{\psi}(E) x+\mu_{\xi}(E)(L-x), \\
& M_{\kappa}=\mu_{\kappa}(E) x+\mu_{\xi}(E)(L-x),
\end{aligned}
$$

which can be defined for both the high and low energy X-rays.

The dual-energy algorithms were applied to this situation using a MATLAB simulation by the author. Figure 6.4(b) and (c) shows the simulated high and low energy $\mathrm{X}$-ray images obtained using equations 6.17. The equivalent thicknesses are found using equation 6.13 at every point on the image. This can be represented as vectors $t_{i}$ on the basis plane, as shown in figure 6.5. Material cancellation is achieved by creating a series of basis projection images (equation 6.15) by varying the angle $\phi$, until the two materials $\psi$ and $\kappa$ appear identical (same projection length along $C$ ) but still contrast with the third material $\xi$ (seen in figure 6.4(d)). This angle is known as the contrast cancellation angle or the look-alike projection angle. On figure 6.5 the basis projection images for cancelling materials $\psi$ and $\kappa$ is the vector at right angles to a straight line joining the two vectors $t_{\psi}$ and $t_{\kappa}$. Contrast cancellation is used to create subtracted 


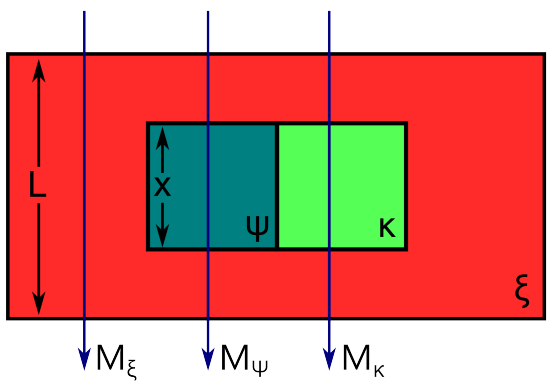

(a)

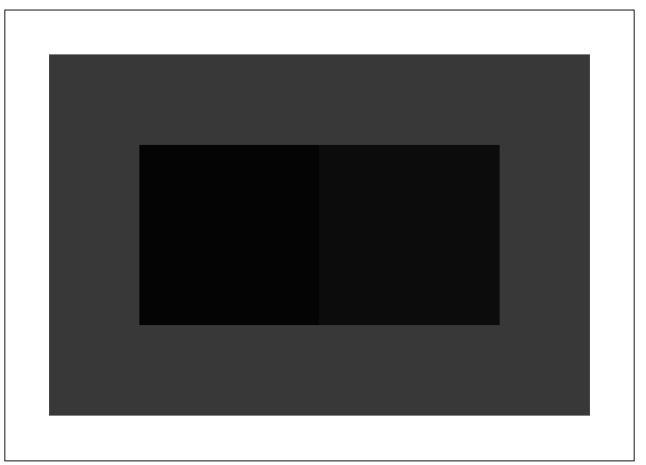

(c)

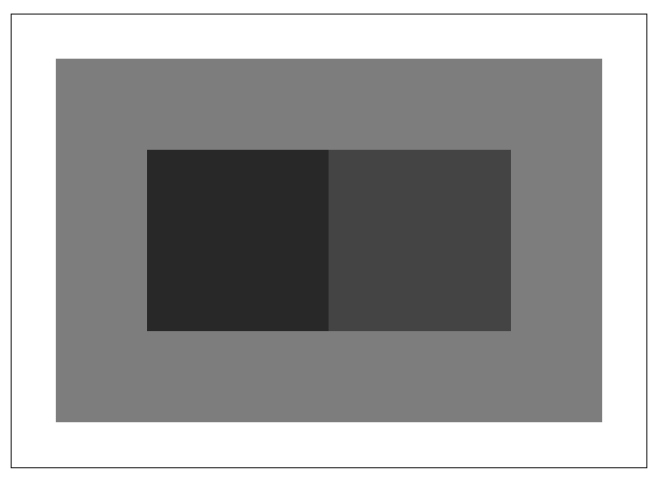

(b)

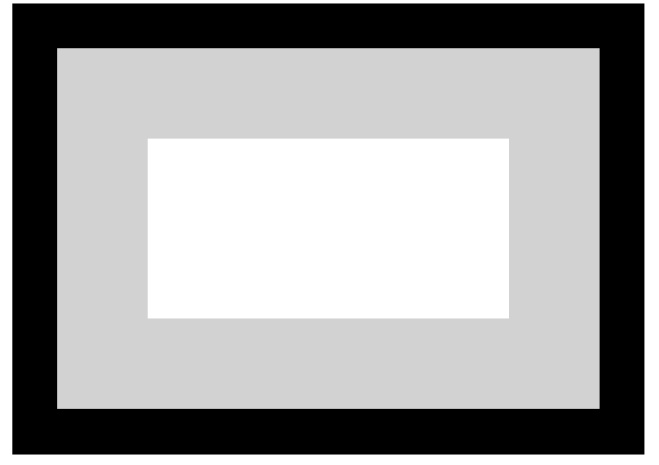

(d)

Figure 6.4: (a) Displacement geometry (side view) and simulated (b) high energy, (c) low energy and (d) cancelled X-ray images. Note, the X-ray images are a top down view.

images, such as those shown in figure 6.1(a)

For mono-energetic X-rays the equivalent thicknesses $A_{\alpha}$ and $A_{\beta}$ can be estimated from the intensity measurements at the two energies. However, X-rays are generally poly-energetic and the logarithmic transmissions are a non linear function of the material thickness. The equivalent thicknesses can be written as a Taylor series expansion,

$$
\begin{aligned}
& A_{\alpha}=k_{1} \log I_{h}+k_{2} \log I_{l}+k_{2} \log ^{2} I_{h}+k_{4} \log ^{2} I_{l}+k_{5} \log I_{l} \log I_{h}, \\
& A_{\beta}=k_{6} \log I_{h}+k_{7} \log I_{l}+k_{8} \log ^{2} I_{h}+k_{9} \log ^{2} I_{l}+k_{10} \log I_{l} \log I_{h} .
\end{aligned}
$$

The $k$ coefficients are determined by least squares fitting the above polynomials during a calibration process with known materials and material thicknesses. A much simpler technique can be applied for subtraction images if the attenuation coefficients of the material to be cancelled are known. Assuming that 


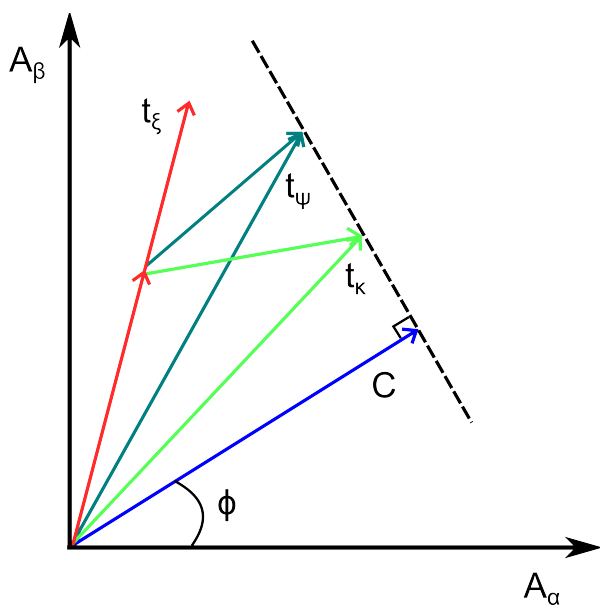

Figure 6.5: Material displacement vectors [79].

mono-energetic $\mathrm{X}$-rays are used, the logarithmic transmissions are given by,

$$
\begin{gathered}
M_{h}=\mu_{a h}\left(E_{h}\right) t_{a}+\mu_{b h}\left(E_{h}\right) t_{b}, \\
M_{l}=\mu_{a l}\left(E_{l}\right) t_{a}+\mu_{b l}\left(E_{l}\right) t_{b} .
\end{gathered}
$$

A composite image can be formed as a linear combination of these two logarithmic transmissions,

$$
M=k_{l} M_{l}+k_{h} M_{h}
$$

with weighting factors $k_{l}$ and $k_{h}$. Substituting equations 6.22 and 6.23 into 6.24 and rearranging gives,

$$
M=\left(k_{l} \mu_{a l}\left(E_{l}\right)+k_{h} \mu_{a h}\left(E_{h}\right)\right) t_{a}+\left(k_{l} \mu_{b l}\left(E_{l}\right)+k_{h} \mu_{b h}\left(E_{h}\right)\right) t_{b} .
$$

Material cancellation can be achieved by setting the coefficient of $t_{i}$ to zero, for example,

$$
k_{l} \mu_{a l}+k_{h} \mu_{a h}=0
$$

which occurs when,

$$
\frac{k_{h}}{k_{l}}=-\frac{\mu_{a l}}{\mu_{a h}}
$$




\subsection{Dual energy imaging with doped CsBr imaging plates}

Current storage phosphor dual-energy imaging techniques use the one-shot technique but each image is read out separately by physically shifting/rotating the imaging plate - a two-step read-out process. It would be advantageous to have a one-step read-out process in order to eliminate registration errors when processing the images. A novel approach to this is based on the idea of a dual layer imaging plate comprised of transparent PSL materials with different emission wavelengths. The images in both layers may be simultaneously recorded and separated using the RGB colour channels of the digital camera read out system described in chapter 4 .

The required structure of the dual-layered imaging plate is shown in figure 6.6. The top layer predominantly absorbs the low energy X-rays, and has a PSL emission at a particular wavelength, for example in the green. The second layer receives the residual higher energy $X$-rays and has an emission at a distinct wavelength from the first layer, eg. in the blue. The two emission wavelengths are then detected in different colour channels of the digital camera, giving the high and low energy images required for dual-energy. For detection of the lower layer emission, the top layer must be optically transparent, in order to minimise scattering and absorption of the PSL light from the lower layer. This

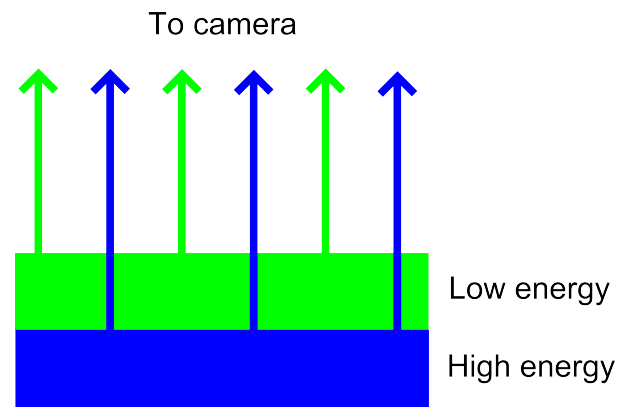

Figure 6.6: Schematic showing dual-layered imaging plate for single-shot, single read-out dual-energy imaging. 
is only possible with the transparent storage phosphors developed here, not with the standard powder phosphors.

The energy separation of the two images arises from the absorption of the poly-energetic X-ray beam by the first layer, taking advantage of the high attenuation of X-rays by $\mathrm{CsBr}$, and the K-edge located at $36 \mathrm{keV}$. Figure 6.7 shows the simulated X-ray energy spectra produced by both a $70 \mathrm{keV}$ and 150 $\mathrm{keV}$ tungsten tube, and also shows the X-ray absorption for each layer. Both layers were assumed to be 300 microns thick. The X-ray spectra are generated by "SpekCalc" [13] and the attenuation coefficients calculated by the XCON database [80]. For the $70 \mathrm{keV}$ energy spectrum (figure 6.7(a)] the X-rays have a mean energy of $36 \mathrm{keV}$, with the mean energy absorbed in the first layer being 35 $\mathrm{keV}$ and $41 \mathrm{keV}$ in the second. This energy separation, at $6 \mathrm{keV}$, is not adequate for dual-energy imaging. The mean energy for a $150 \mathrm{keV}$ poly-energetic $\mathrm{X}$-ray beam is $56 \mathrm{keV}$. The mean energy absorbed in the first layer is $44 \mathrm{keV}$ and the second is $59 \mathrm{keV}$, giving an energy difference of $15 \mathrm{keV}$. This should be an adequate energy separation for dual-energy imaging.

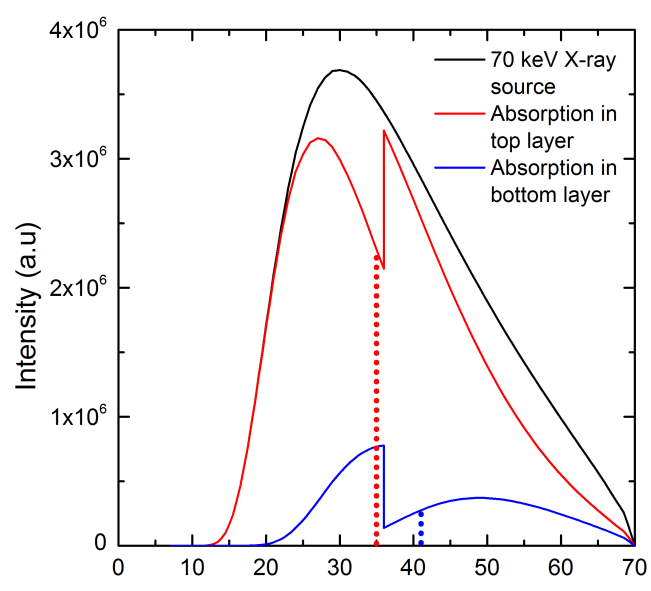

(a)

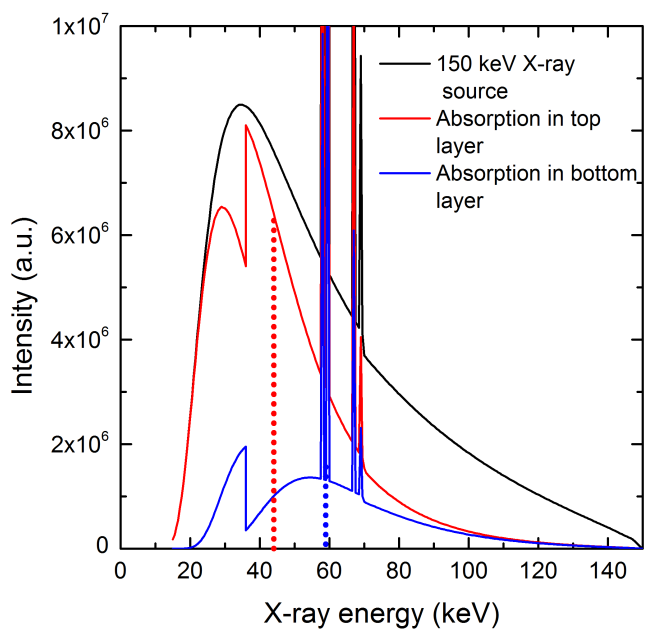

(b)

Figure 6.7: Incident and absorbed X-ray energy spectra for (a) $70 \mathrm{keV}$ and (b) $150 \mathrm{keV}$ poly-energetic $\mathrm{X}$-ray beams. The dotted line shows the mean absorbed energy in each layer. 


\subsubsection{Possible dopants for colour-separable PSL}

An alternative colour dopant, apart from the usual blue-emitting $\mathrm{Eu}^{2+}$ for $\mathrm{CsBr}$, is required for the second layer of the dual-layer imaging plate. Previous work by Hangleiter et al. [23] and Rogulis et al. [37] found CsBr doped with monovalent gallium $\left(\mathrm{Ga}^{+}\right)$or indium $\left(\mathrm{In}^{+}\right)$showed a storage phosphor effect. In the literature both of these dopants have an emission in the green region of the spectrum, peaking at $504 \mathrm{~nm}$ and $515 \mathrm{~nm}$ for indium and gallium respectively. Along with the two mentioned dopants, samarium was also trialled, as $\mathrm{Sm}^{2+}$ has a known red emission.

\section{Gallium}

$\mathrm{CsBr}: \mathrm{Ga}^{+}$imaging plates were prepared by first melting the $\mathrm{CsBr}$ powder at 650 ${ }^{\circ} \mathrm{C}$ and adding a 2:1 mixture of $\mathrm{GaBr}_{3}$ and Ga metal [37] to the melt following the supposed reaction,

$$
\mathrm{GaBr}_{3}+2 \mathrm{Ga} \rightarrow 3 \mathrm{Ga}^{+}+3 \mathrm{Br}^{-}
$$

The melt was cooled to room temperature, either by quenching or a rapid cool down. The melt straight out of the furnace is clear but over a period of time ranging between 2 - 12 hours develops an orange colouration. This orange coloured CsBr:Ga+ has an absorbance peak between 400 and $500 \mathrm{~nm}$ which disappears upon heating to $250^{\circ} \mathrm{C}$.

CsBr: $\mathrm{Ga}^{+}$made in this fashion has a broadband PSL emission peaking at approximately $515 \mathrm{~nm}$, which is shown in figure 6.8. The conversion efficiency

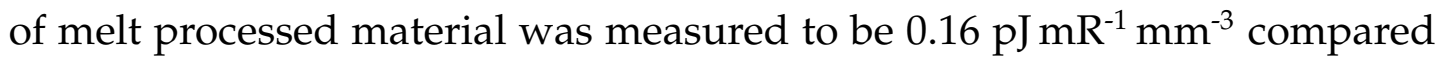
to $1.5 \mathrm{pJ} \mathrm{mR}^{-1} \mathrm{~mm}^{-3}$ for an as-made (non hydrated) $\mathrm{CsBr}: \mathrm{Eu}^{2+}$ imaging plate. The conversion efficiency was measured using a $810 \mathrm{~nm}$ infrared LED rather than the standard $633 \mathrm{~nm}$ LED because the green emission is broadband and overlaps with the spectrum of the $633 \mathrm{~nm}$ LED. 


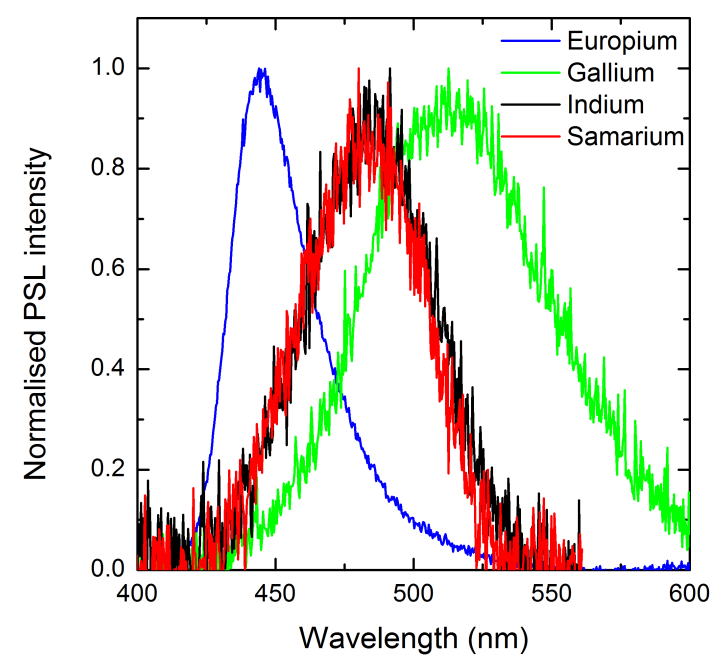

Figure 6.8: PSL from possible colour dopants as compared to europium doped materials.

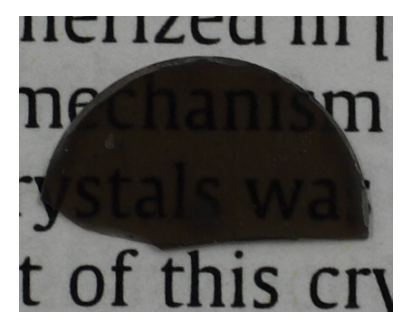

Figure 6.9: Optical appearance of $\mathrm{CsBr}: \mathrm{Ga}^{+}$imaging plate.

When milled and sieved the powder has a grey appearance, and when pressed leads to a very dark pellet which is only slightly transparent (figure 6.9). This leads to a conversion efficiency which is too small to be measured. The black colour is likely caused by an excess of gallium metal which is localised in the melt material but which is distributed though the powder during milling. The metal absorbs the PSL emission leading to the low CE. No further work was therefore attempted with this material.

\section{Indium}

Indium in the form of $\mathrm{In}^{+}$is also known to give green PSL emission [23]. Powdered CsBr:In ${ }^{+}$was prepared as described by Thoms et al. [81]. $\mathrm{CsBr}$ and $\mathrm{InCl}_{3}$ precursors were made into an aqueous solution by adding enough distilled water to just dissolve the powders. This was heated at approximately $130{ }^{\circ} \mathrm{C}$ over a period of $12-18$ hours to remove the water. The powder was then 


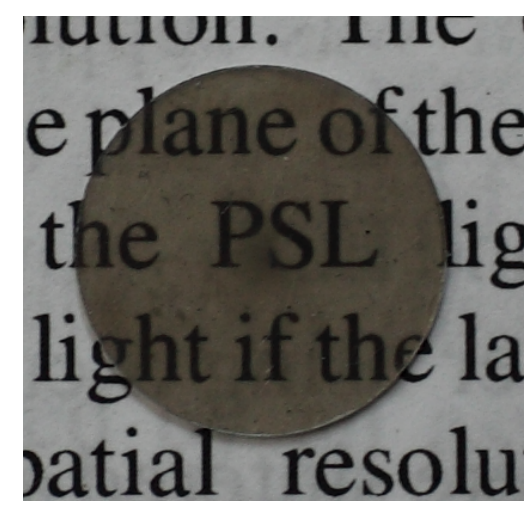

(a)

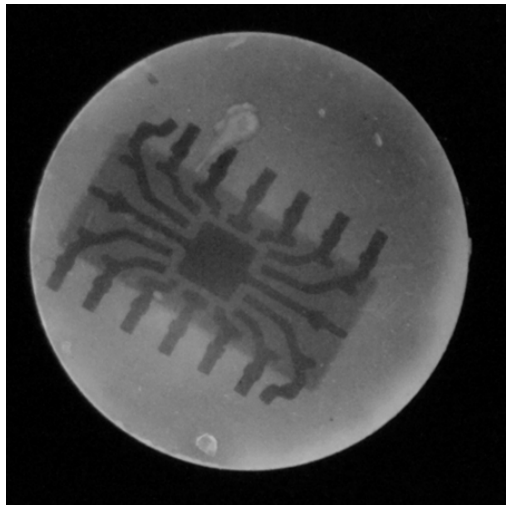

(b)

Figure 6.10: (a) Optical appearance and (b) X-ray image of IC chip of as-made CsBr:In ${ }^{+}$imaging plate.

heated to $500{ }^{\circ} \mathrm{C}$ in a reducing atmosphere (argon gas with $5 \%$ hydrogen) and held there for 2 hours to convert the $\mathrm{In}^{3+}$ to $\mathrm{In}^{+}$. The resulting powder was milled, sieved and pressed into imaging plates. The as-made imaging plates have good transparency and are able to produce X-ray images, as seen in figure 6.10. The PSL has a very broad emission band peaking at approximately 485 $\mathrm{nm}$, with a conversion efficiency measured to be $0.16 \mathrm{pJ} \mathrm{mR}^{-1} \mathrm{~mm}^{-3}$. Hydrating the imaging plate increases the conversion efficiency (to $0.5 \mathrm{pJ} \mathrm{mR}^{-1} \mathrm{~mm}^{-3}$ for 5 minutes hydration) as was seen with europium doped imaging plates.

\section{Samarium}

In an attempt to achieve red $\mathrm{Sm}^{2+}$ PSL emission, CsBr powder was mixed with $\mathrm{SmBr}_{3}$, and melted at $650{ }^{\circ} \mathrm{C}$ in a reducing atmosphere (argon with 5 $\%$ hydrogen). The melted mixture showed PSL, again broadband and peaking at approximately $485 \mathrm{~nm}$, with a very low conversion efficiency of 0.002 $\mathrm{pJ} \mathrm{mR} \mathrm{mm}^{-1} \mathrm{~mm}^{-3}$. The emission wavelength is surprising as samarium usually shows red luminescence in the divalent state and blue luminescence in the trivalent state, but has no known green emission. Also surprising is the PSL spectrum, shown in figure 6.8, which is identical for both samarium and indium. This suggests the luminescence is not due to the dopant ion, but some form of intrinsic luminescence from the CsBr. Again, when milled and sieved the pow- 
der takes on a grey appearance, and any pellets are again of low optical quality as for the gallium-doped imaging plates. This leads to the pellets showing no measurable PSL.

The indium doped imaging plates show the best combination of optical quality and PSL performance. The layered structure therefore consisted of an indium doped and europium doped imaging plate.

\subsubsection{Imaging}

Dual-layered imaging plates were irradiated using a Golden Engineering XR150 portable X-ray generator which produces a poly-energetic $\mathrm{X}$-ray spectrum from a tungsten anode with a maximum energy of $150 \mathrm{keV}$. The $\mathrm{X}$-ray generator to imaging plate distance was approximately $100 \mathrm{~mm}$. After irradiation the duallayered imaging plate was transferred to the camera read-out system (described in chapter 4 in the dark. The dual-layer imaging plate was read out either side-by-side, analogous to the one-shot method described earlier, or as a layered structure where the energy separation is by the colour channels of the digital camera. Stimulation was by a $810 \mathrm{~nm}$ infrared LED, to which the digital camera is insensitive (see chapter 4.2), and therefore the images are recorded with no additional filtering other than the inherent camera filtration. The images were saved in RAW format before being converted into TIFF images as described in chapter 4 and processed using MATLAB.

\section{Separate read-out}

A dual-layered imaging plate consisting of two $300 \mu \mathrm{m}$ thick europium doped $\mathrm{CsBr}$ imaging plates was used to test the validity of dual-energy imaging with a layered structure as both the detector and energy filter. Both layers were not subjected to any deliberate hydration or annealing. The two images were obtained by irradiating as a layered structure and then placing the top layer beside the lower for read out. Europium doped imaging plates were used for 


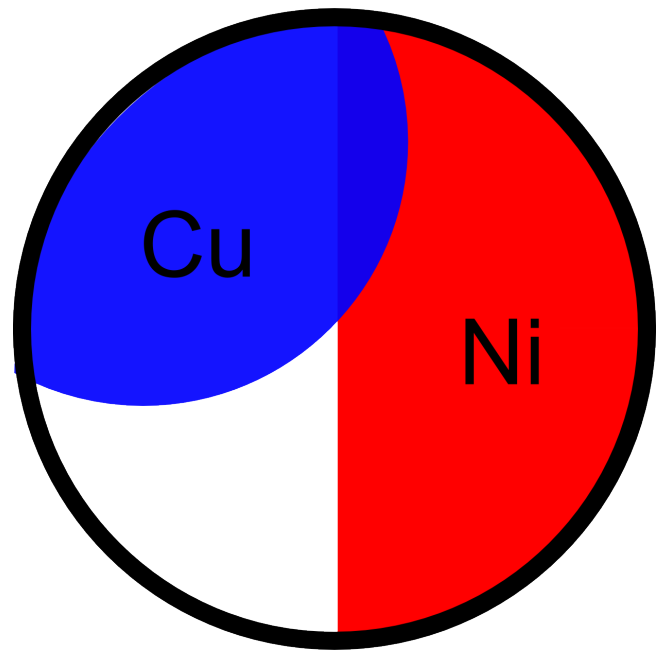

(a)

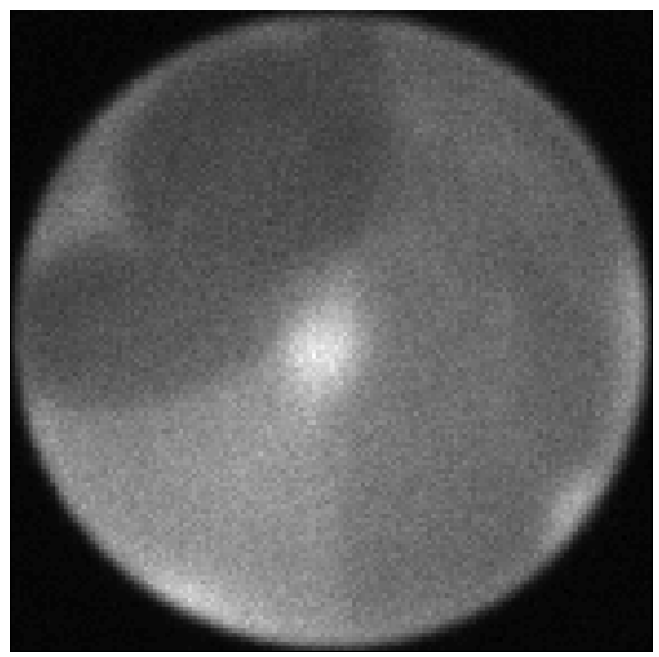

(c)

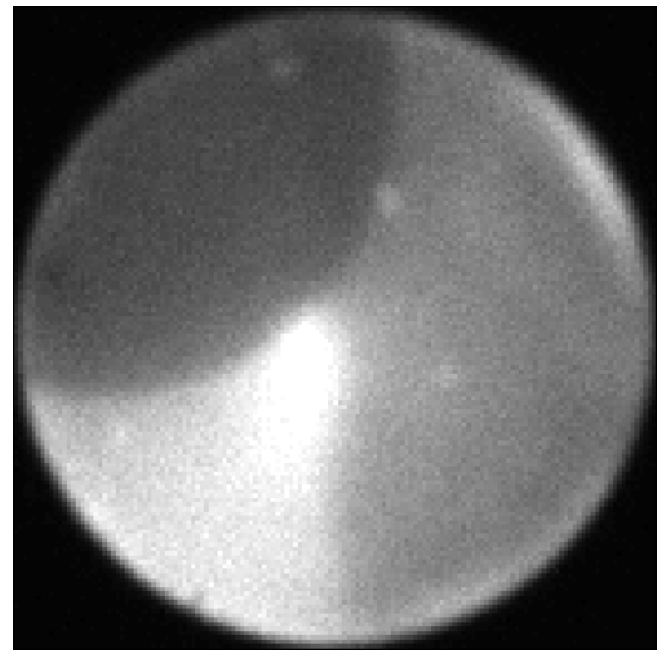

(b)

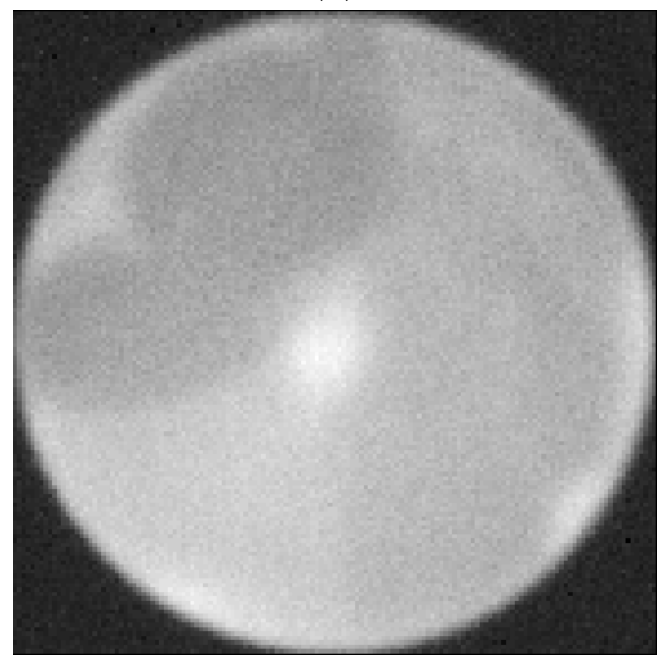

(d)

Figure 6.11: (a) Schematic of target and, (b) low energy, (c) high energy and (d) processed image taken with a dual-layered $\mathrm{CsBr}: \mathrm{Eu}^{2+}$ imaging plate.

this test as they have a higher PSL light output compared to the indium doped imaging plates.

Figure 6.11 shows high and low energy images obtained with the above process, and a material cancellation image. The high and low energy images were cropped and rotated for registration so that dual-energy algorithms could be applied. For implementation of the dual-energy algorithms it was assumed that the two images were obtained with two mono-energetic X-ray beams. The two energies chosen were $45 \mathrm{keV}$ and $60 \mathrm{keV}$ for the low and high energy beam respectively, as these were approximately the values determined by simulation (see section 6.2). The object which was X-rayed was a copper disc and nickel 
Table 6.1: Table of basis material parameters used in dual-energy algorithms.

\begin{tabular}{ccccc}
\hline \multirow{2}{*}{ Parameter } & \multicolumn{2}{c}{ Titanium $(\alpha)$} & \multicolumn{2}{c}{ Copper $(\beta)$} \\
& Low & High & Low & High \\
\hline$Z$ & 22 & 22 & 29 & 29 \\
$Z / A$ & 0.4595 & 0.4595 & 0.4564 & 0.4564 \\
$\rho\left(\mathrm{g} \mathrm{cm}^{-3}\right)$ & 4.54 & 4.54 & 8.96 & 8.96 \\
$\mu\left(\mathrm{cm}^{-1}\right)$ & 7.287 & 3.478 & 21.342 & 14.273 \\
\hline
\end{tabular}

sheet, therefore, the basis materials were chosen to be titanium $(Z=22)$ and copper $(Z=29)$, as they encompass the range of common $3 d$ metals. For these materials and energies the algorithm parameters are given in table 6.1.

Attempts at determining the material composition were unsuccessful, giving values of $Z=23$ for copper and $Z=24$ nickel compared to the actual values of 29 and 28 respectively. Nevertheless, material cancellation was achieved with these imaging plates. The equivalent thicknesses $\left(A_{\alpha}\right.$ and $\left.A_{\beta}\right)$ were calculated for every point in the image using equation 6.13. 91 projection images (equation 6.15) were made by varying the angle $\phi$ in one degree steps. Examination of the projection images determined the angles at which material cancellation has been achieved. Figure 6.11(d) shows the projection image with $\phi=78^{\circ}$, where partial cancellation between the nickel and the clear area can be seen.

\section{Dual read-out}

The dual-layered imaging plate used for this method consisted of an europium doped bottom layer and an indium doped top layer, both with a thickness of $300 \mu \mathrm{m}$. The indium was used as the top layer as its CE is much lower than for europium doped materials, and the bottom layer receives a much lower $X$-ray intensity. The two imaging plates were read out simultaneously, and then separated into the high and low energy images by taking the blue and green channel images respectively. The high and low energy images for a copper disc and nickel sheet can be seen in figure 6.12(a) and (b). Again material composition was unreliable although in the correct order, giving values for $\mathrm{Z}$ of 


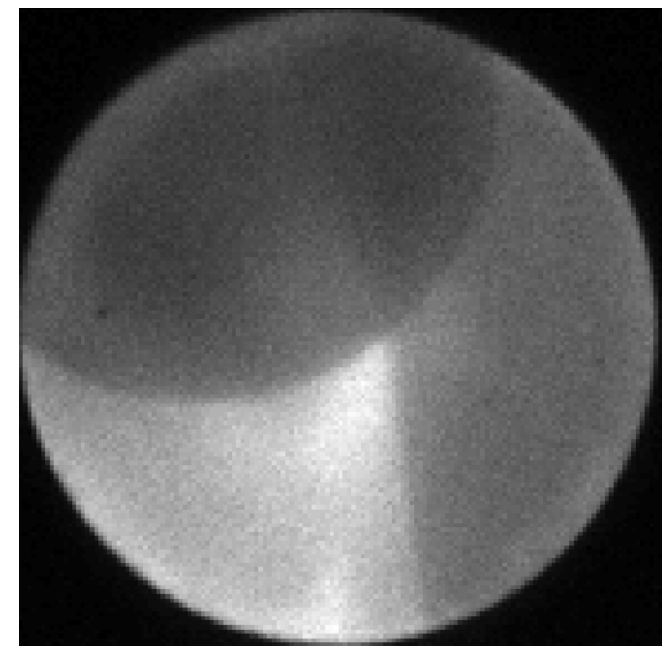

(a)

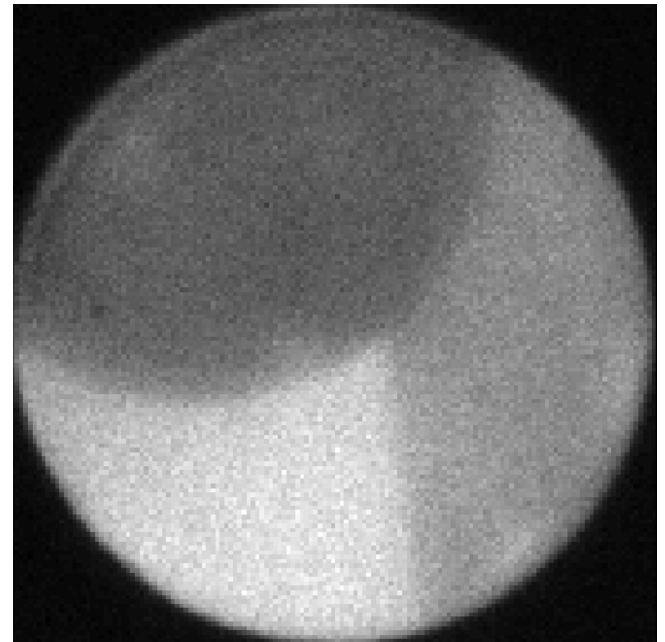

(b)

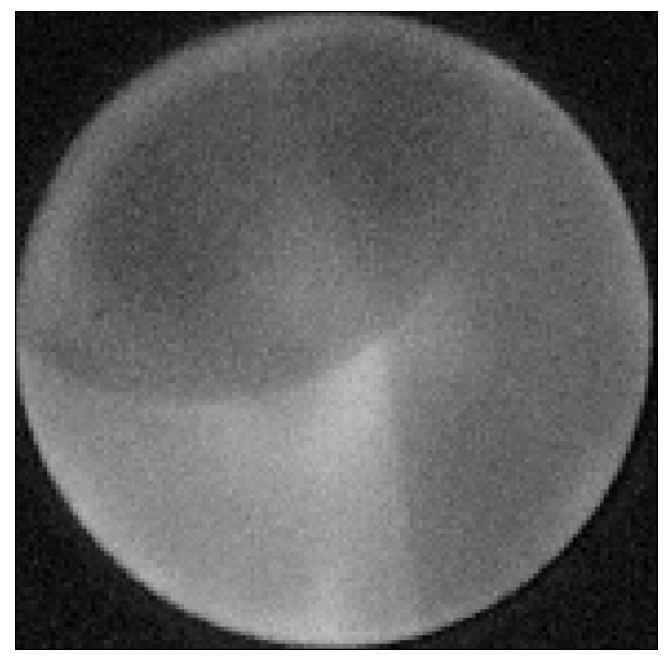

(c)

Figure 6.12: (a) Low energy, (b) high energy, and (c) processed X-ray images taken with a dual-layered $\mathrm{CsBr}$ imaging plate consisting of an indium doped top layer and europium doped bottom layer.

43 and 32 for copper and nickel respectively. The two images were processed as for the previous case, and the resulting image at $\phi=72^{\circ}$ is shown in figure 6.12(c) Partial cancellation is achieved between the copper and nickel.

\subsection{Discussion}

CsBr:In ${ }^{+}$imaging plates have a PSL emission peaking at $485 \mathrm{~nm}$ and a low conversion efficiency. Further experiments suggest that this luminescence may not in fact be due to the indium dopant, but rather some intrinsic luminescence 
of $\mathrm{CsBr}$. Undoped $\mathrm{CsBr}$ treated in the same manner as for samarium doping (melting at $650{ }^{\circ} \mathrm{C}$ in an argon/hydrogen atmosphere), also shows PSL peaking at approximately $485 \mathrm{~nm}$ as can be seen in figure 6.13. This luminescence potentially comes from impurities in the CsBr (such as oxygen [32]) which are converted to an active PSL site during the heating process.

Further studies could be performed on monovalent gallium doped imaging plates as a potential second dopant. The as-melted mixture has a PSL emission peaking at $515 \mathrm{~nm}$, and a conversion efficiency of $0.16 \mathrm{pJ} \mathrm{mR}^{-1} \mathrm{~mm}^{-3}$. Although the $\mathrm{CE}$ is low, the colour separation between this dopant and the europium is much better than for indium. Currently this dopant, as a melting mixture, has a spontaneous orange colouration which appears over a short period of time. Heating to approximately $250{ }^{\circ} \mathrm{C}$ returns the colour to its original state. This colouration and removal with heat treatment has been seen previously by Zazubovich et al. [82]. Mikhal'chenko et al. [83] suggest that spontaneous colouration in $\mathrm{CsBr}$ single crystals is from colloidal particles, most probably consisting of elemental gallium. Additionally, when milled and sieved the powder has a grey appearance, which when pressed gives a very absorbing imaging plate. This grey colouration of the powder and pellet is most likely due to excess gallium metal being added to the melt, and so it would be worthwhile to experiment with lower amounts of gallium metal dopant.

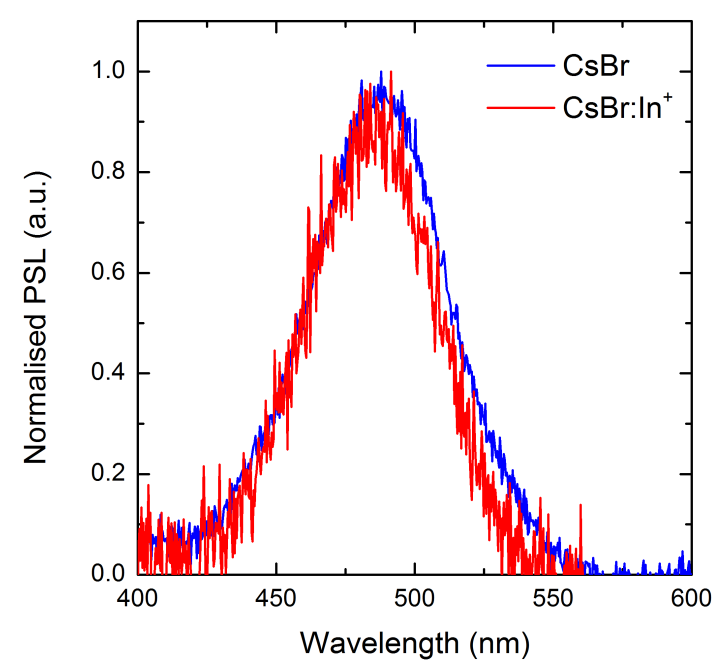

Figure 6.13: PSL of undoped and indium doped CsBr imaging plates. 
Stimulation of the imaging plates in this chapter was by an $810 \mathrm{~nm}$ LED. This wavelength stimulation was used as the usual $633 \mathrm{~nm}$ LED overlapped with the green dopant during PSL measurement, and also it meant no filtration was required for the camera read out technique. Testing the $810 \mathrm{~nm}$ LED with a 5 minute hydrated $\mathrm{CsBr}: \mathrm{Eu}^{2+}$ imaging plate gave a conversion efficiency of 9.3 pJ mR $\mathrm{mm}^{-1} \mathrm{~mm}^{-3}$ compared to $8.6 \mathrm{pJ} \mathrm{mR}^{-1} \mathrm{~mm}^{-3}$ for the $633 \mathrm{~nm}$ LED. However, 810 nm stimulation is on the edge of the F-centre absorption band which leads to the decay time of the PSL being very long. Previously the decay time for the $633 \mathrm{~nm}$ LED with the 5 minute hydrated sample was found to be $0.6 \mathrm{~s}$, but for the same imaging plate with $810 \mathrm{~nm}$ stimulation the decay time is 10.3 seconds. This requires a longer exposure time with the camera, leading to additional noise in the image.

When applying the dual-energy algorithms it was assumed that the images were created with two mono-energetic X-ray beams with energies equal to the mean energies of the poly-energetic X-ray beam absorbed in each layer. However, as the X-ray beam used was actually poly-energetic, and the actual energies absorbed by both layers was not known, the algorithms should be adjusted for this. A simple approach to this was given in section 6.1 by decomposing the equivalent thicknesses into a Taylor series involving the logarithmic transmissions at high and low energies (equation 6.20). This requires a calibration process where the logarithmic transmissions for materials with known thickness were least squares fitted to the Taylor series. Unfortunately, applying this to a simple simulation (the simplest cases being mono-energetic X-rays) gave incorrect values for the equivalent thicknesses, and hence, attempts at determining material composition were unsuccessful.

Dual-energy imaging with layered CsBr imaging plates doped with europium and indium has been investigated. Initial indications show that it is possible to perform dual-energy imaging with these layered structures. The energy separation between the two layers was calculated to be approximately $15 \mathrm{keV}$ 
without any additional filtration. This separation is adequate for dual-energy imaging as Ishigaki et al. [84] were able to perform dual-energy analysis in the medical context using an energy separation of approximately $8-15 \mathrm{keV}$. In their study the layered imaging plate detector consisted of two $\mathrm{BaFBr}: \mathrm{Eu}^{2+}$ imaging plates separated by a $0.2-0.4 \mathrm{~mm}$ copper filter. In the technique described here an increased energy separation could be achieved by using a transparent intervening layer, such as lead-doped glass. Improvements in the dual-layer single read-out technique are needed in terms of the colour separation of the two dopants. Further work could investigate the use of the poly-energetic forms of the dual-energy algorithms, as the current representation of broad energy spectra by a single energy reduces the effectiveness of the technique. 


\section{Chapter 7}

\section{Neutron imaging with CsBr:Eu ${ }^{2+}$ imaging plates}

Neutron imaging is a technique similar to shadow radiographic X-ray imaging, in the sense that an image is created by detecting the attenuation of neutrons through an object. Neutrons are strongly attenuated through scattering from hydrogen-rich objects, such as water and hydrocarbons, and can give complementary information to that obtained by X-ray imaging.

This thesis is solely concerned with thermal neutron imaging. Thermal neutrons have an energy of $25 \mathrm{meV}$ at room temperature (energy at most probable speed) and are produced by slowing down (or moderating) the fast neutrons generated in a nuclear reactor. The attenuation of thermal neutrons though a material is governed by a Beer-Lambert type law,

$$
I=I_{0} \exp (-N \sigma z)
$$

where $N$ is the concentration of target atoms, $\sigma$ the capture or scattering crosssection and $z$ the thickness of the material. Capture cross-sections as a function of atomic number for thermal neutrons are shown in figure 7.1 . The typical detectors used with thermal neutron imaging are not particularly sensitive to neutrons, so instead a converter material containing elements which 


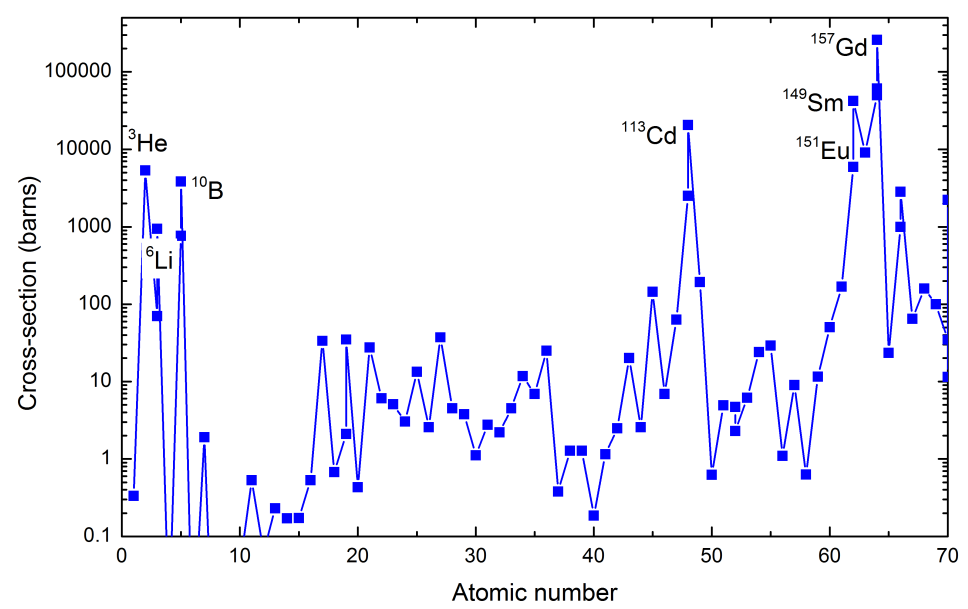

Figure 7.1: Neutron capture cross-sections as a function of atomic number [85].

have a high capture cross-section is first used to convert the neutrons to gamma rays or electrons. Typically these elements are highly enriched isotopes, some of which are shown in table 7.1.

Traditionally neutron images were recorded on photographic film with the use of a converter screen typically composed of a gadolinium component. This technique has now been replaced by digital methods with higher sensitivities and higher dynamic range [86]. These systems are analogous to those for X-rays (see chapter 1) and include imaging plate systems and scintillators coupled to flat panel detectors.

Commercial neutron imaging plates, made by Afga or Fuji for example, consist of a powder mixture of $\mathrm{BaFBr}: \mathrm{Eu}^{2+}$ co-doped with $\mathrm{Gd}_{2} \mathrm{O}_{3}$ (gadolinium oxide) embedded in an organic binder. In the imaging plate the gadolinium converter

Table 7.1: Thermal neutron capture reactions for isotopes with a high capture cross-section [16]. 1 barn $=10^{-24} \mathrm{~cm}^{2}$, "ce" = conversion electron.

\begin{tabular}{|c|c|c|c|}
\hline Element & $\begin{array}{c}\text { Abundance } \\
(\%)\end{array}$ & Capture reaction & $\begin{array}{l}\text { Cross section } \\
\quad \text { (barns) }\end{array}$ \\
\hline${ }^{3} \mathrm{He}$ & 0.00014 & ${ }^{3} \mathrm{He}+{ }^{1} \mathrm{n} \rightarrow{ }^{3} \mathrm{H}+{ }^{1} \mathrm{p}+0.77 \mathrm{MeV}$ & 5333 \\
\hline${ }^{6} \mathrm{Li}$ & 7.5 & ${ }^{6} \mathrm{Li}+{ }^{1} \mathrm{n} \rightarrow{ }^{3} \mathrm{H}+{ }^{4} \mathrm{He}+4.79 \mathrm{MeV}$ & 940 \\
\hline${ }^{10} \mathrm{~B}$ & 20 & $\begin{array}{c}{ }^{10} \mathrm{~B}+{ }^{1} \mathrm{n} \rightarrow{ }^{7} \mathrm{Li}+{ }^{4} \mathrm{He}+2.78 \mathrm{MeV}(7 \%) \\
\rightarrow{ }^{7} \mathrm{Li}^{*}+{ }^{4} \mathrm{He}+\gamma(93 \%)\end{array}$ & 3837 \\
\hline${ }^{155} \mathrm{Gd}$ & 14.8 & ${ }^{155} \mathrm{Gd}+{ }^{1} \mathrm{n} \rightarrow{ }^{156} \mathrm{Gd}+\gamma+\mathrm{ce}$ s & 61000 \\
\hline${ }^{157} \mathrm{Gd}$ & 15.7 & ${ }^{157} \mathrm{Gd}+{ }^{1} \mathrm{n} \rightarrow{ }^{158} \mathrm{Gd}+\gamma+\mathrm{ce}$ s & 254000 \\
\hline
\end{tabular}




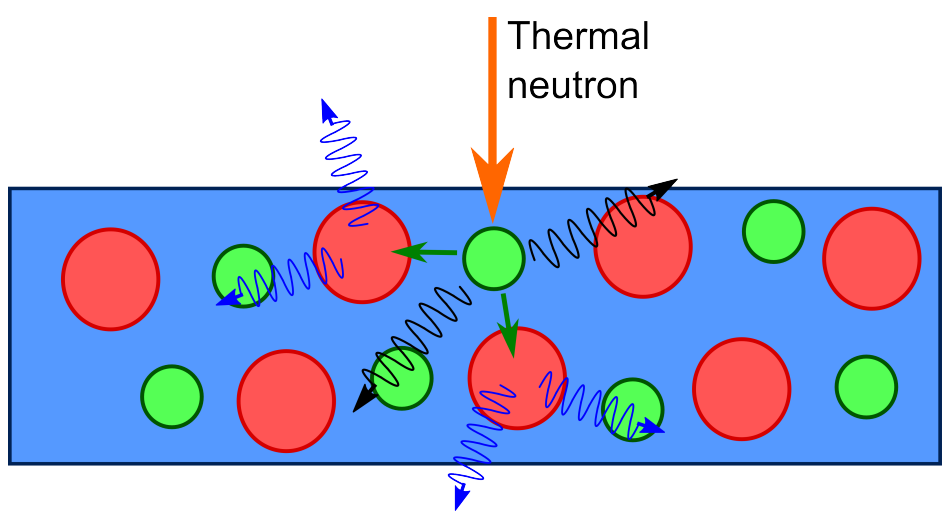

Figure 7.2: Schematic of neutron conversion and PSL in storage phosphors, where the red circles are phosphor particles, green circles neutron converter particles, green lines conversion electrons, black lines gamma rays and blue lines PSL emission.

particles are mixed with the phosphor particles as shown in figure 7.2. The gadolinium converter ion captures a neutron and emits both gamma rays and conversion electrons following the capture reactions in table 7.1. The emitted gamma rays can have energies up to $7.5 \mathrm{MeV}$, and are likely to exit the imaging plate without interaction; at this energy the half thicknesses for $\mathrm{BaFBr}$ and $\mathrm{Gd}_{2} \mathrm{O}_{3}$ are 94 and $23 \mathrm{~mm}$ respectively. The conversion electrons are much more likely to interact with the imaging plate as they have an energy range of 0.03 $0.2 \mathrm{MeV}$ and interact strongly with the electrons of the plate atoms through the Coulomb interaction. With these energies the electrons travel range is between $0.7-15 \mu \mathrm{m}$ for $\mathrm{BaFBr}$ and $0.5-11 \mu \mathrm{m}$ for $\mathrm{Gd}_{2} \mathrm{O}_{3}$ [87]. The conversion electrons transfer their energy to the phosphor particles where the PSL process takes place and light is emitted. As with X-ray storage phosphor imaging plates, the resolution is limited by scattering of the stimulation light during the read-out phase.

Amorphous silicon flat panel detectors coupled with a neutron sensitive scintillator are also used for neutron imaging. Common scintillator layers consist of gadolinium oxysulphide, which takes advantage of the high absorption of neutrons by gadolinium, or zinc sulphide with added ${ }^{6} \mathrm{Li}$ as the neutron converter. Currently this system has a lower dynamic range than the neutron detecting storage phosphor imaging plates [86]. 
In this chapter, the development of neutron imaging plates based on $\mathrm{CsBr}: \mathrm{Eu}^{2+}$ is discussed. The aim was to make high resolution transparent imaging plates by co-doping with neutron sensitive compounds containing lithium, boron or gadolinium. Thermal neutron irradiations took place at the OPAL research reactor.

\subsection{OPAL research reactor}

The Open Pool Australian Lightwater (OPAL) reactor is a nuclear research reactor located at the Australian Nuclear Science and Technology Organisation (ANSTO) headquarters in Sydney, Australia [88]. OPAL is a multi-purpose facility which provides neutron beams for research or industrial applications and produces radiopharmaceuticals for the diagnosis or treatment of cancer or other diseases.

A nuclear reactor produces neutrons by controlled fission reactions. In a fission reaction, a neutron absorbed by a large atomic nucleus (such as uranium-235

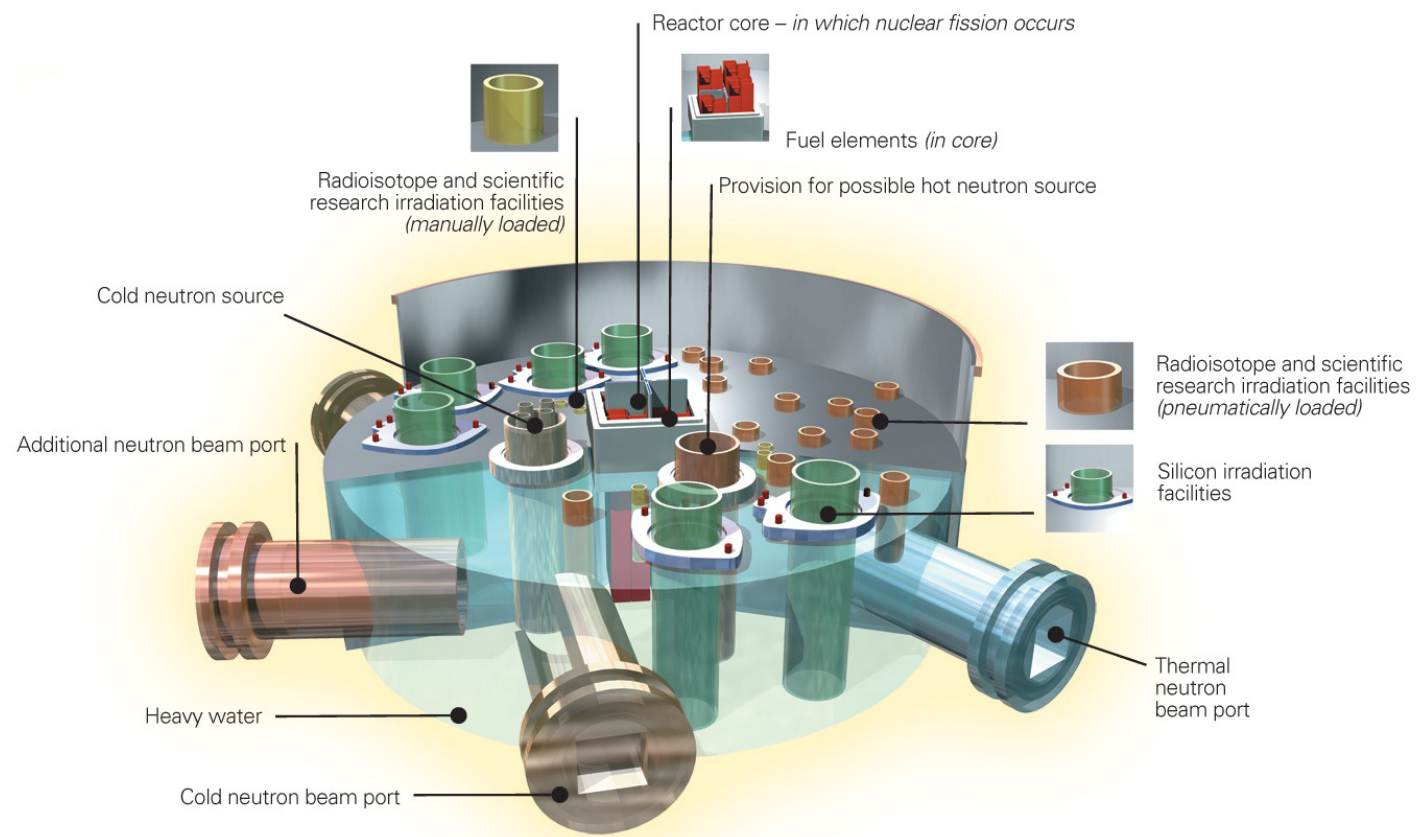

Figure 7.3: Cut away diagram of OPAL reflector vessel [88]. The thermal neutron beam port was used to irradiate imaging plates. 
or plutonium-239) causes the nucleus to split into two lighter nuclei, releasing kinetic energy, gamma radiation and free neutrons. In a nuclear reactor the fission reaction is sustained by moderating some of the free neutrons and allowing them to react with more nuclei. In nuclear power plants it is the kinetic energy which is harvested to produce power, whereas in a research reactor it is the neutrons which are of importance.

The OPAL reactor consists of a reflector vessel (figure 7.3) which is located near the bottom of a 13 metre deep open pool filled with demineralised (ordinary) light water. The water in the pool is used as a coolant for the reactor core which is situated in the middle of the reflector vessel.

The reactor core consists of 16 fuel assemblies in a four by four matrix, with each containing 21 uranium fuel plates (20\% uranium-235 and $80 \%$ uranium-238). Spaced amongst the fuel assemblies are five hafnium control rods, which are used to absorb excess neutrons and control the reaction. Neutrons are produced by the many uranium-235 reactions of the general form [89],

$$
{ }^{235} U+n \rightarrow X+Y+2.5 n,
$$

where the $X$ and $Y$ are fission fragments or atoms of smaller atomic weight and the $2.5 n$ is the average number of neutrons released. One example of a ${ }^{235} \mathrm{U}$ reaction is,

$$
n+{ }_{92}^{235} U \rightarrow{ }_{92}^{236} U \rightarrow{ }_{56}^{144} \mathrm{Ba}+{ }_{36}^{89} \mathrm{Kr}+3 n+177 \mathrm{MeV}
$$

which produces three neutrons for every one neutron used in the reaction. The reaction vessel itself is made of a zirconium alloy and measures $2.6 \mathrm{~m}$ diameter and $1.2 \mathrm{~m}$ high. The interior of the vessel is filled with heavy water $\left(\mathrm{D}_{2} \mathrm{O}\right)$ which acts as a moderator and reflects some of the neutrons back into the core to maintain the chain reaction. The heavy water also moderates the fast neutrons produced in the above reaction to create both thermal and "cold" 
(energy of 0.05 to $25 \mathrm{meV}$ ) neutrons. Also contained in the reaction vessel are irradiation targets (for creating radioisotopes and research irradiations) and various neutron beam ports which make the neutrons available for research applications away from the core. Currently both the thermal and "cold" neutron beam lines are active. Examples of instruments using the neutron beams are powder and Laue diffractometers, small angle neutron scattering instruments and stress diffractometers.

The thermal neutron beam line used for the irradiations detailed in this chapter was the "Koala" beam line which is used for a Laue diffractometer. The advantage of this instrument was the ability to pass the neutron beam straight through the instrument and irradiate samples directly. The neutron flux is approximately $2.5 \times 10^{6}$ neutrons $\mathrm{cm}^{-2} \mathrm{~s}^{-1}$ at the instrument.

The OPAL research reactor was visited on two separate occasions to test developmental $\mathrm{CsBr}$ neutron imaging plates with the thermal neutron beam line.

\subsection{CsBr neutron imaging plates}

$\mathrm{CsBr}: \mathrm{Eu}^{2+}$ is a high performing $\mathrm{X}$-ray storage phosphor imaging plate, but as of yet there is no commercial neutron imaging plate based on this material. Neutron converters in the form of lithium, boron and gadolinium compounds were added to $\mathrm{CsBr}: \mathrm{Eu}^{2+}$ imaging plates to make them neutron sensitive. In these imaging plates the converter compound captures the neutron and emits alpha particles or conversion electrons which are absorbed by the phosphor, the energy of which is used in the PSL process. This is similar to figure 7.2, except the imaging plate consists of only phosphor particles and converter ions (there is no binder material). An efficient PSL process requires,

1. the reaction products to escape from the neutron converter,

2. the reaction products to be absorbed by the $\mathrm{CsBr}: \mathrm{Eu}^{2+}$ phosphor, and

3. the PSL to escape from the imaging plate with little light scattering or 
absorption due to refractive index mismatch, or absorption.

The work that is presented in this chapter was carried out before the comprehensive study on $\mathrm{CsBr}: \mathrm{Eu}^{2+}$ imaging plates was completed (see chapter 5). Therefore, the optimal preparation process for these imaging plates had not been developed, and as a consequence, the neutron imaging plates are of modest optical quality, particularly for the first visit.

\subsubsection{First visit}

A total of ten imaging plates with various europium dopant concentrations or neutron converter compounds were tested. Two groups of imaging plates were prepared, the first contained no converter material (apart from $\mathrm{Eu}^{2+}$ itself), but a variable $\mathrm{Eu}^{2+}$ concentration. The second held the $\mathrm{Eu}^{2+}$ concentration fixed at $0.2 \mathrm{~mol} \%$, but with a variety of converter compounds in various fractions of $\mathrm{CsBr}$. The imaging plates were prepared as described in chapter 3.1, that is, the required powders are mixed and ground in a ball mill to less than $25 \mu \mathrm{m}$ grain size and pressed into $13 \mathrm{~mm}$ diameter pellets. No pre-sintering of the powders was performed. Imaging plates were subsequently annealed for 10 minutes at $150{ }^{\circ} \mathrm{C}$ in an argon atmosphere. The imaging plates were not subject to deliberate hydration, but were prepared in the original dry box with modest humidity levels ( $\sim 25 \mathrm{ppm})$. The incorporation of $\mathrm{Eu}^{2+}$ into the $\mathrm{CsBr}$ therefore takes place by the mechanism discussed in chapter 5 .

The PSL intensity when irradiated with both X-rays and neutrons was measured with a custom read-out unit designed and built at Victoria University [90]. In this read-out system a $0.1 \mathrm{~mW} 633 \mathrm{~nm}$ laser diode stimulates the luminescence which is detected by a photomultiplier tube with a BG12 filter to block the stimulating light. Not all of the light emitted is detected, therefore this is a relative, not absolute, measurement technique. Measurements were taken with respect to a standard commercial Fuji $\mathrm{BaFBr}$ neutron imaging plate. 


\section{Europium-only doping}

Natural europium is composed of two isotopes, Eu-151 and Eu-153, with Eu-151 having an abundance of $47.8 \%$ and a high neutron capture cross-section of 9100 barns. Eu-151 captures a neutron and forms Eu-152 in a highly excited state, which then decays by primary and secondary gamma ray cascades; particle emission is possible but this is less likely as Eu-152 has a long half life (13.5 years), though it may occur with high energy neutrons. Neutron PSL in europium-only doped materials relies on the secondary gamma rays having low enough energy (10s of $\mathrm{keV})$ to be absorbed in the imaging plate. The gamma ray emission from $\mathrm{Eu}-152$ is in the range of $40-1400 \mathrm{keV}$ [91], therefore some absorption will occur (approximately half the gamma rays are absorbed for 40 $\mathrm{kV}$ gamma rays and a $300 \mu \mathrm{m}$ thick CsBr imaging plate).

Imaging plates doped only with $\mathrm{EuBr}_{2}$ were first tested as a potential neutron imaging plate, with the europium concentration varied with each imaging plate. The composition and thickness for each imaging plate is given in table 7.2 . The PSL intensity per unit thickness for the europium only doped imaging plates is given figure 7.4(a) for both $\mathrm{X}$-rays and thermal neutrons. For neutrons the intensity is expressed as a percentage of a commercial Fuji neutron imaging plate; the X-ray PSL intensity scale is arbitrary but normalised for thickness.

Under X-ray irradiation the thinnest (1 \% Eu) imaging plate has the highest PSL intensity per unit thickness. This imaging plate has a thickness of $0.36 \mathrm{~mm}$

Table 7.2: Composition and thickness for CsBr imaging plates doped with various $\mathrm{Eu}^{2+}$ concentrations and no additional neutron converter for the first visit.

\begin{tabular}{llc}
\hline Name & Composition & Thickness (mm) \\
\hline $0.2 \% \mathrm{Eu}$ & CsBr:0.2 mol\% $\mathrm{EuBr}_{2}$ & 0.64 \\
$0.5 \% \mathrm{Eu}$ & $\mathrm{CsBr}: 0.5 \mathrm{~mol} \% \mathrm{EuBr}_{2}$ & 1.05 \\
$1 \% \mathrm{Eu}$ & $\mathrm{CsBr}: 1.0 \mathrm{~mol} \% \mathrm{EuBr}_{2}$ & 1.06 \\
$1 \% \mathrm{Eu}$ & $\mathrm{CsBr}: 1.0 \mathrm{~mol} \% \mathrm{EuBr}_{2}$ & 0.36 \\
$2 \% \mathrm{Eu}$ & $\mathrm{CsBr}: 2.0 \mathrm{~mol} \% \mathrm{EuBr}_{2}$ & 1.06 \\
\hline
\end{tabular}




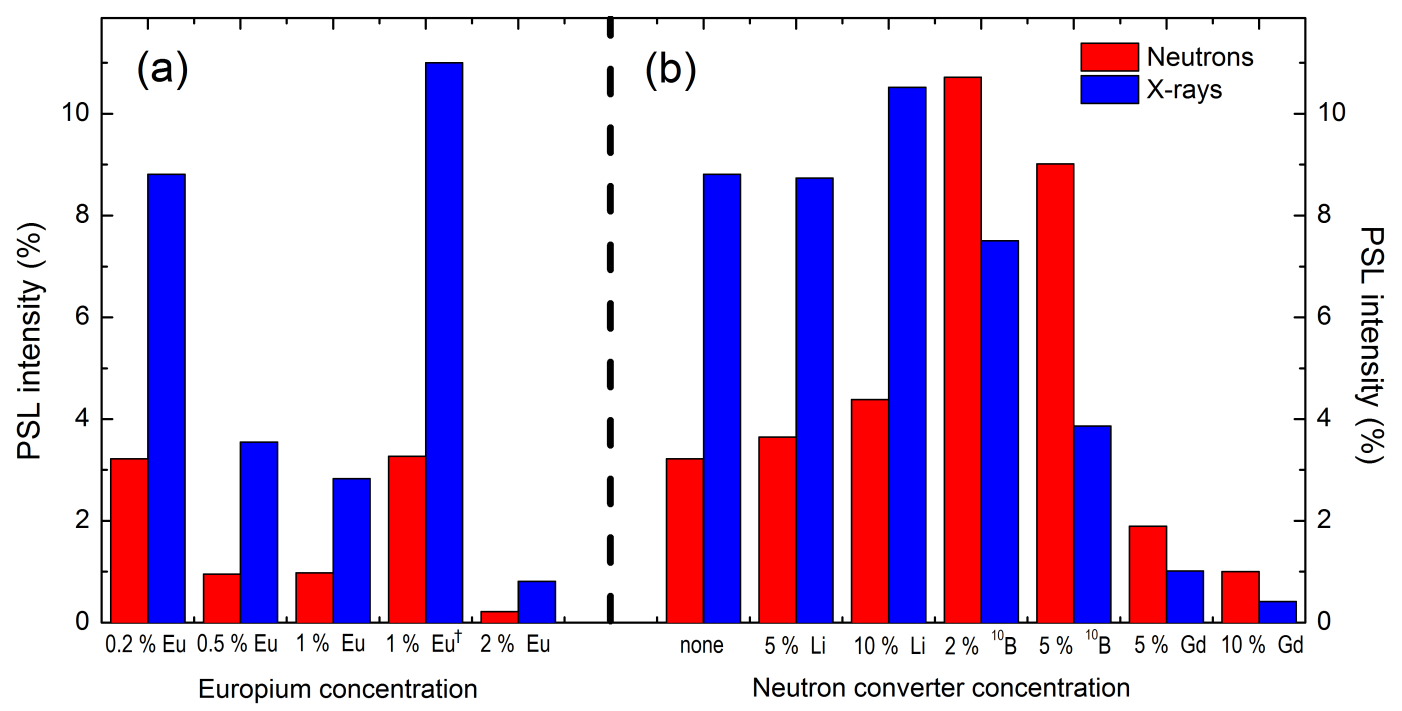

Figure 7.4: Neutron and X-ray PSL intensity scaled with thickness for (a) imaging plates doped with various europium concentrations, and (b) fixed europium concentration $(0.2 \mathrm{~mol} \%)$ with various additional neutron converters. The PSL intensity is as a percentage of the PSL of the commercial Fuji neutron imaging plate. The X-ray PSL intensity scale is arbitrary.

as compared to $1.06 \mathrm{~mm}$ for a second $1 \%$ Eu imaging plate which shows a similar PSL intensity, leading to the thinner sample having a higher PSL per unit thickness. The $0.2 \%$ Eu imaging plate is also thin, at $0.66 \mathrm{~mm}$, and shows the next highest X-ray PSL of the europium only doped imaging plates. The optical quality of the thinner samples is much better than the thicker samples (1 mm samples are relatively opaque) which suggests that in thicker imaging plates some of the emission light is not escaping the sample and instead being scattered and re-absorbed, which reduces the PSL signal.

In terms of neutron PSL, the $0.2 \% \mathrm{Eu}$ and the thin $1 \% \mathrm{Eu}$ imaging plates show an intensity which is approximately $3 \%$ of the commercial Fuji imaging plate. These show a relatively high PSL considering none of the usual neutron converters are used.

\section{Lithium, boron, and gadolinium converters}

Imaging plates were also produced with a fixed $0.2 \%$ europium concentration and additional neutron converters in the form of (natural) $\mathrm{LiBr}$, enriched ${ }^{10} \mathrm{~B}_{2} \mathrm{O}_{3}$ and (natural) $\mathrm{GdBr}_{3}$. The compositions of these imaging plates can be found 
Table 7.3: Composition and thickness for $\mathrm{CsBr}: \mathrm{Eu}^{2+}$ imaging plates doped with $0.2 \mathrm{~mol} \% \mathrm{Eu}$ and various neutron converter materials for the first visit.

\begin{tabular}{llc}
\hline Dopant & Composition & Thickness (mm) \\
\hline $5 \% \mathrm{LiBr}$ & $\mathrm{CsBr}: 0.2 \mathrm{~mol} \% \mathrm{EuBr}_{2}: 5.0 \mathrm{~mol} \% \mathrm{LiBr}$ & 0.65 \\
$10 \% \mathrm{LiBr}$ & $\mathrm{CsBr}: 0.2 \mathrm{~mol} \% \mathrm{EuBr}_{2}: 10.0 \mathrm{~mol} \% \mathrm{LiBr}$ & 0.62 \\
$2 \%{ }^{10} \mathrm{~B}_{2} \mathrm{O}_{3}$ & $\mathrm{CsBr}: 0.2 \mathrm{~mol} \% \mathrm{EuBr}_{2}: 2.0 \mathrm{~mol} \%{ }^{10} \mathrm{~B}_{2} \mathrm{O}_{3}$ & 0.78 \\
$5 \%{ }^{10} \mathrm{~B}_{2} \mathrm{O}_{3}$ & $\mathrm{CsBr}: 0.2 \mathrm{~mol} \% \mathrm{EuBr}_{2}: 5.0 \mathrm{~mol} \%{ }^{10} \mathrm{~B}_{2} \mathrm{O}_{3}$ & 0.85 \\
$5 \% \mathrm{GdBr}_{3}$ & $\mathrm{CsBr}: 0.2 \mathrm{~mol} \% \mathrm{EuBr}_{2}: 5.0 \mathrm{~mol} \% \mathrm{GdBr}_{3}$ & 0.79 \\
$10 \% \mathrm{GdBr}_{3}$ & $\mathrm{CsBr}: 0.2 \mathrm{~mol} \% \mathrm{EuBr}_{2}: 10.0 \mathrm{~mol} \% \mathrm{GdBr}_{3}$ & 0.82 \\
\hline
\end{tabular}

in table 7.3. The X-ray and neutron PSL intensities per unit thickness are shown on the right of figure 7.4, with the $0.2 \%$ Eu-only doped imaging plate results reproduced for comparison. All the imaging plates with added neutron converters had a poor optical quality (white opaque appearance) which is caused by the mismatch of the refractive indices. The refractive index of $\mathrm{CsBr}$ is approximately 1.7 at $440 \mathrm{~nm}$ [45] compared to $1.8,1.6$ and 1.9 for $\mathrm{LiBr}, \mathrm{B}_{2} \mathrm{O}_{3}$ [92] and $\mathrm{GdBr}_{3}$ respectively (where the refractive index of $\mathrm{GdBr}_{3}$ has not been measured, but is assumed to be similar to $\mathrm{LaBr}_{3}[93]$ ]).

The X-ray PSL intensities for the $0.2 \%$ Eu-doped imaging plates are the same within uncertainties in the Eu concentration (due to weighing) with or without the $\mathrm{LiBr}$ converter, therefore the $\mathrm{LiBr}$ is not interfering with the PSL process. For the boron and gadolinium doped imaging plates the X-ray PSL intensity is lower than for the plate without a converter material. This suggests that either the converter is interfering with the PSL mechanisms in the CsBr which would require some atomic diffusion, or more likely, the high opacity of the imaging plate is preventing some of the emitted light from escaping.

Under neutron irradiation the enriched boron converter imaging plates show the highest PSL intensity, at approximately $11 \%$ and $9 \%$ of the commercial plate for the $2 \%$ and $5 \%$ convertor fraction imaging plates respectively. This higher PSL is due to the boron-10 ions effectively capturing thermal neutrons and producing short range alpha particles for the PSL process. The lithium doped samples show relatively good PSL at around $4 \%$ for both the $5 \%$ and $10 \%$ 
converter fractions. These imaging plates were doped with $\mathrm{LiBr}$ which contains natural lithium, of which only $7.5 \%$ is the required lithium- 6 isotope. Therefore, this PSL intensity may be increased by using an enriched lithium- 6 compound, potentially by a factor of $100 / 7.5 \approx 13$. The gadolinium converter imaging plates show poor PSL intensities under both X-ray and neutron irradiation which is most likely due to the reduced optical quality resulting in only a small fraction of the PSL light being emitted.

\subsubsection{Second visit}

The compositions of the imaging plates prepared for the second visit are shown in table 7.4. Neutron converters used were enriched lithium-6 carbonate, boron10 oxide and gadolinium bromide with natural Gd isotope abundances. The imaging plates were prepared as described previously, and for each converter both a hydrated ( $1 \mathrm{~min}$ at $100 \% \mathrm{RH})$ and an annealed $\left(10 \mathrm{~min}\right.$ at $200{ }^{\circ} \mathrm{C}$ in an argon atmosphere) imaging plate were prepared. The latter plates were not subject to any deliberate hydration. The europium only doped and lithium converter imaging plates where of a much higher optical quality than the first generation, which led to more measurements being taken. Measurements of the

Table 7.4: Composition, and thickness for europium only doped imaging plates and imaging plates doped with $0.2 \mathrm{~mol} \% \mathrm{Eu}$ and various neutron converter materials for the second visit.

\begin{tabular}{llc}
\hline Sample & Composition & Thickness (mm) \\
\hline $0.2 \% \mathrm{Eu}$ & CsBr: $0.2 \mathrm{~mol} \% \mathrm{EuBr}_{2}$ & 0.37 \\
$0.5 \% \mathrm{Eu}$ & $\mathrm{CsBr}: 0.5 \mathrm{~mol} \% \mathrm{EuBr}_{2}$ & 0.37 \\
$1 \% \mathrm{Eu}$ & $\mathrm{CsBr}: 1.0 \mathrm{~mol} \% \mathrm{EuBr}_{2}$ & 0.39 \\
$1 \%{ }^{6} \mathrm{Li}$ & $\mathrm{CsBr}: 0.2 \mathrm{~mol} \% \mathrm{EuBr}_{2}: 1.0 \mathrm{~mol} \%{ }^{6} \mathrm{Li}_{2} \mathrm{CO}_{3}$ & 0.37 \\
$2 \%{ }^{6} \mathrm{Li}$ & $\mathrm{CsBr}: 0.2 \mathrm{~mol} \% \mathrm{EuBr}_{2}: 2.0 \mathrm{~mol} \%{ }^{6} \mathrm{Li}_{2} \mathrm{CO}_{3}$ & 0.40 \\
$5 \%{ }^{6} \mathrm{Li}$ & $\mathrm{CsBr}: 0.2 \mathrm{~mol} \% \mathrm{EuBr}_{2}: 5.0 \mathrm{~mol} \%{ }^{6} \mathrm{Li}_{2} \mathrm{CO}_{3}$ & 0.35 \\
$2 \%{ }^{10} \mathrm{~B}$ & $\mathrm{CsBr}: 0.2 \mathrm{~mol} \% \mathrm{EuBr}_{2}: 2.0 \mathrm{~mol} \%{ }^{10} \mathrm{~B}_{2} \mathrm{O}_{3}$ & 0.35 \\
$5 \%{ }^{10} \mathrm{~B}$ & $\mathrm{CsBr}: 0.2 \mathrm{~mol} \% \mathrm{EuBr}_{2}: 5.0 \mathrm{~mol} \%{ }^{10} \mathrm{~B}_{2} \mathrm{O}_{3}$ & 0.37 \\
$2 \% \mathrm{Gd}$ & $\mathrm{CsBr}: 0.2 \mathrm{~mol} \% \mathrm{EuBr}_{2}: 2.0 \mathrm{~mol} \% \mathrm{GdBr}_{3}$ & 0.39 \\
$5 \% \mathrm{Gd}$ & $\mathrm{CsBr}: 0.2 \mathrm{~mol} \% \mathrm{EuBr}_{2}: 5.0 \mathrm{~mol} \% \mathrm{GdBr}_{3}$ & 0.41 \\
\hline
\end{tabular}


UV-Vis and infrared absorbance (for europium doped and lithium converter imaging plates only), and the PSL intensity for both X-ray and neutron irradiation were performed. Separate samples were prepared for the X-ray and neutron PSL measurements.

Figure 7.5 shows the optical extinction spectra for the $0.5 \% \mathrm{Eu}$ and the $1 \%{ }^{6} \mathrm{Li}$ imaging plates, in the as-made (red curve), annealed (green curve) and hydrated (blue curve) condition. As-made, the $0.5 \%$ europium-only imaging plate has a transmission coefficient of approximately $0.3 \%$ at the emission wavelength of $440 \mathrm{~nm}$. With hydration of the imaging plate the transmission coefficient decreases. For an annealed imaging plate, the transmission coefficient increases to $1.3 \%$ at $440 \mathrm{~nm}$. An increase in transmission was also found in chapter 5 when annealing $\mathrm{CsBr}: \mathrm{Eu}^{2+}$ imaging plates $\left(0.2 \mathrm{~mol} \% \mathrm{Eu}^{2+}\right)$ up to a temperature of $100{ }^{\circ} \mathrm{C}$, and was associated with a decrease in the free water content. The asmade imaging plate containing $0.2 \mathrm{~mol} \% \mathrm{Eu}^{2+}$ and $1 \%{ }^{6} \mathrm{Li}$ converter is relatively opaque compared to the above case, with a transmission coefficient of $0.09 \%$ at $440 \mathrm{~nm}$. This does not significantly change with annealing or hydration.

In these two examples the spectra are dominated by light scattering for $\lambda>400$ nm. The $\mathrm{Eu}^{2+}$ absorption peaks (seen in chapter 5) are only apparent as an edge

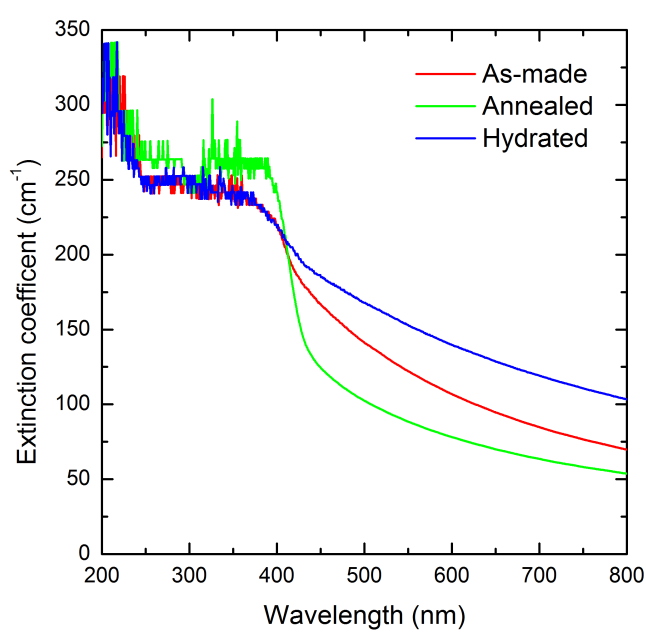

(a)

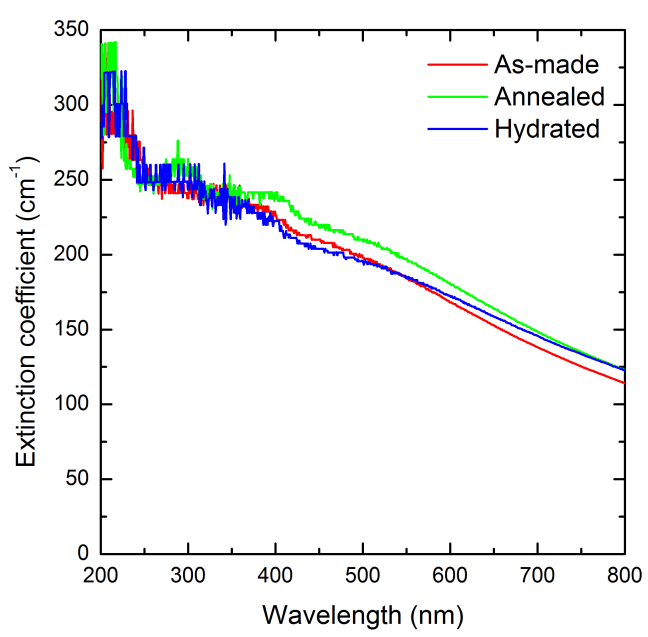

(b)

Figure 7.5: UV-Vis absorbance spectra for (a) a $0.5 \%$ Eu-only doped imaging plate and (b) a $0.2 \mathrm{~mol} \% \mathrm{Eu}^{2+}$ imaging plate with $1 \%{ }^{6} \mathrm{Li}$ as a neutron convertor. 


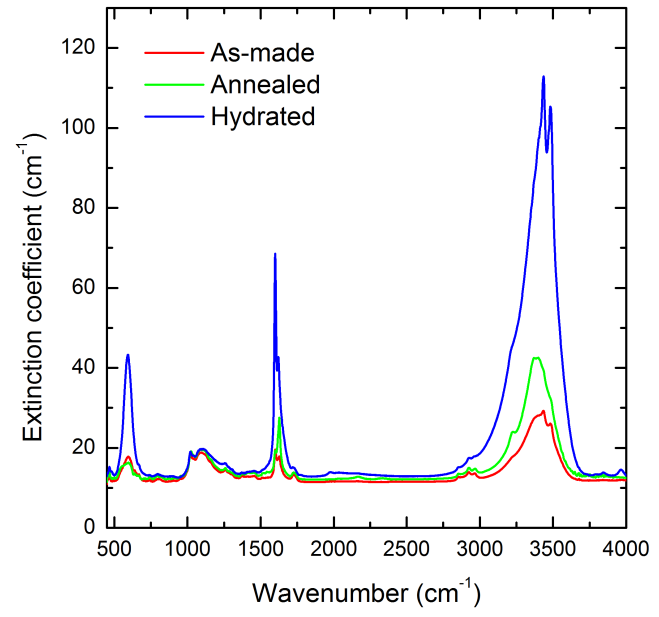

(a)

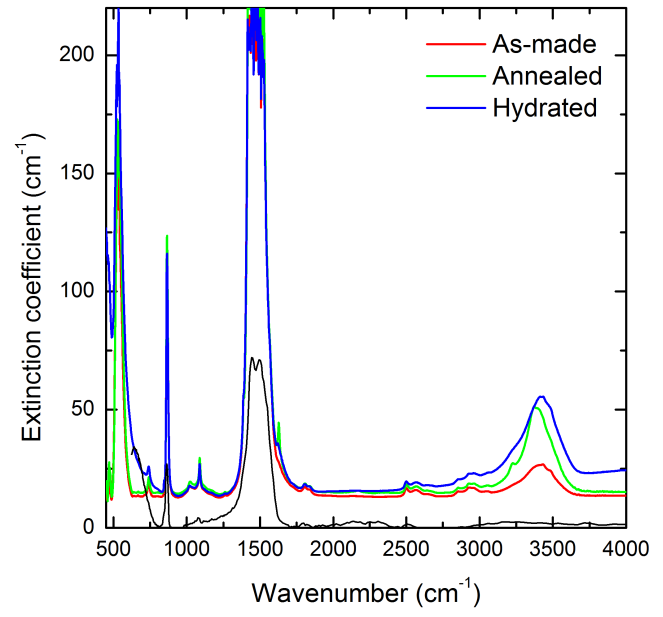

(b)

Figure 7.6: Infrared absorbance spectra for (a) a $0.5 \%$ Eu-only doped and (b) a $0.2 \mathrm{~mol} \% \mathrm{Eu}^{2+}$ imaging plate with $1 \%{ }^{6} \mathrm{Li}$ as a neutron converter. The black curve in (b) is the $\mathrm{Li}_{2} \mathrm{CO}_{3}$ IR spectrum [94].

for the annealed sample in figure 7.5(a) and weak band features between 250 and $400 \mathrm{~nm}$ in all the spectra. The strong scattering in the case of the imaging plates containing ${ }^{6} \mathrm{Li}$ converter is caused by the large mismatch in refractive index between the two materials (approximately 1.7 for $\mathrm{CsBr}$ [45] and 1.4 for $\left.\mathrm{Li}_{2} \mathrm{CO}_{3}[92]\right)$.

The infrared spectra of the same europium only doped imaging plates are shown in figure 7.6(a), The as-made imaging plate has the same character as described in chapter 5 where the sharp peaks superimposed on the $\nu_{1}$ and $\nu_{3}$ free water stretching vibration band, and the double peak of the $\nu_{2}$ bending vibration are due to $\mathrm{EuBr}_{2} \cdot 1 \mathrm{H}_{2} \mathrm{O}$. The 1 minute hydrated imaging plate shows an increase in the $\nu_{1}, \nu_{3}$ and $\nu_{2}$ free water vibrations, indicative of an increase in water content as found previously. However, in this case the peaks due to $\mathrm{EuBr}_{2} \cdot 1 \mathrm{H}_{2} \mathrm{O}$ are much stronger than those seen for a 1 minute hydrated 0.2 $\mathrm{mol} \% \mathrm{Eu}^{2+}$ doped imaging plate seen previously (figure 5.7). This suggests that with a higher $\mathrm{EuBr}_{2}$ content there is a higher concentration of $\mathrm{EuBr}_{2} \cdot 1 \mathrm{H}_{2} \mathrm{O}$ as is expected. These results suggest that the as-made and hydrated imaging plates will show a lower PSL intensity than the annealed imaging plate since europium is still present in the non-PSL active $\mathrm{EuBr}_{2} \cdot 1 \mathrm{H}_{2} \mathrm{O}$ form. 
The IR spectra for the imaging plate with $1 \%{ }^{6} \mathrm{Li}$ (figure $7.6(\mathrm{~b})$ ) shows different characteristics to the europium only doped imaging plates. The spectra in this case are dominated by peaks corresponding to $\mathrm{Li}_{2} \mathrm{CO}_{3}$ (shown as the black curve in figure $7.6(\mathrm{~b})$ ). These peaks do not change significantly with annealing or hydration of the imaging plates. The $\nu_{1}, \nu_{3}$ stretching vibration bands show small $\mathrm{EuBr}_{2} \cdot 1 \mathrm{H}_{2} \mathrm{O}$ peaks in the as-made and hydrated condition, not clearly visible in figure 7.6(b), which have disappeared for the annealed imaging plate. The $\nu_{2}$ vibration cannot be seen under the main $\mathrm{Li}_{2} \mathrm{CO}_{3}$ peak.

\section{X-ray PSL}

Figure 7.7(a) shows the X-ray PSL intensity per unit thickness in the as-made (red), annealed (green) and hydrated (blue) condition for each dopant. These intensities were measured using the integrating sphere method described in chapter 3.3 . The europium-only doped imaging plates show a general trend of increasing PSL with europium concentration but in a sub-linear fashion.

The as-made and annealed $1 \%{ }^{6} \mathrm{Li}$ imaging plates (containing $0.2 \%$ europium) show a higher PSL intensity than the $0.2 \mathrm{~mol} \%$ europium-only doped imaging plate. This is likely caused by some lithium ions being incorporated in the CsBr lattice (by thermomechanical transfer as in chapter 5), which is somehow stabilising the PSL active sites. The hydrated imaging plate shows a similar PSL intensity to the equivalent europium-only imaging plate. The other lithium concentrations show similar or lower PSL intensities, which in the latter case is probably due to the decreasing optical quality of the imaging plates.

Enriched boron-10 doped imaging plates showed very little/no PSL as made, and hydration could not significantly increase the PSL intensity. However, annealing the imaging plates was found to increase the PSL intensity to values higher than for the singly doped $0.2 \%$ Eu sample.

Gadolinium doped imaging plates show a lower PSL intensity for both converter fractions as compared to the $0.2 \%$ Eu-only imaging plate, suggesting the 


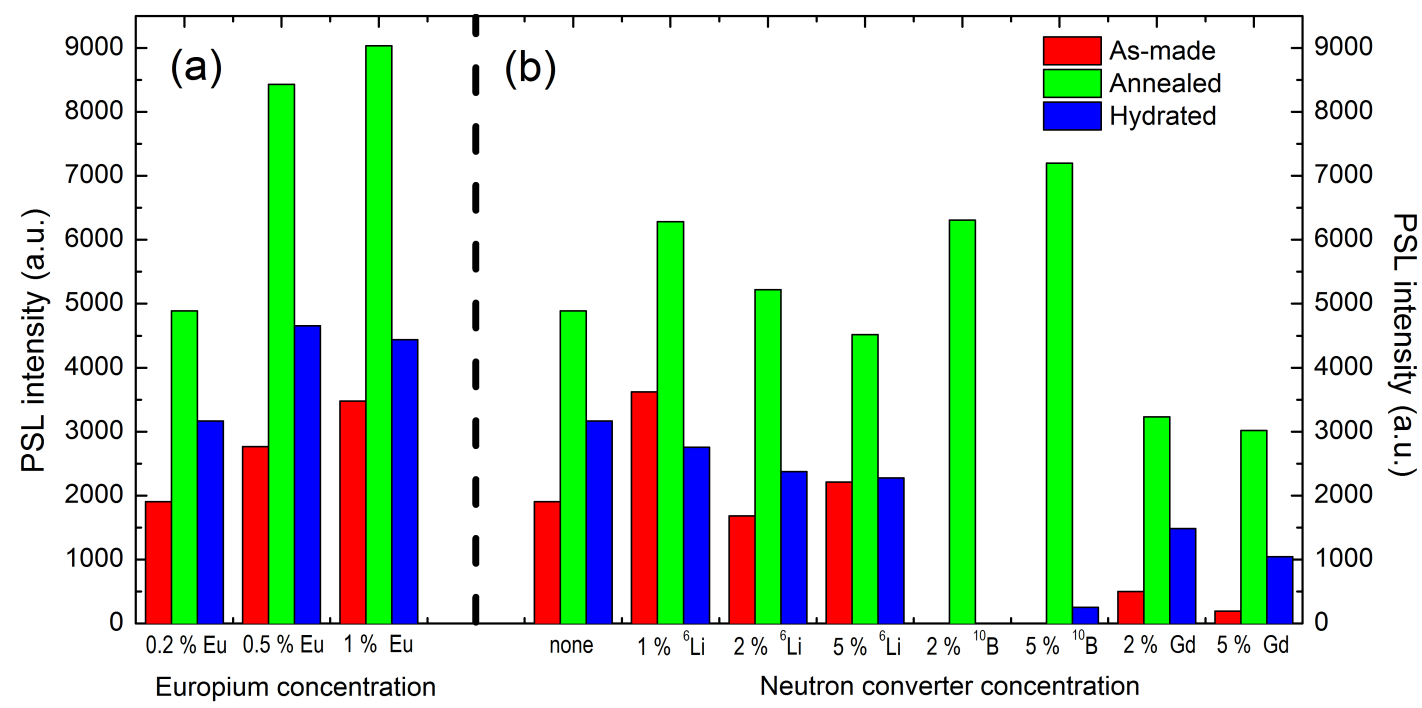

Figure 7.7: X-ray PSL intensities for as-made, annealed and hydrated imaging plates for all dopant types. The intensity scale is arbitrary and no bar corresponds to the signal being too small to measure.

poor optical quality of these imaging plates is causing the emitted light to be re-absorbed.

\section{Neutron PSL}

Neutron PSL intensities were measured using the camera system described in chapter 4 . The integration sphere setup was not used as the CCD spectrometer developed an electronic fault during this visit which made it inoperable. The PSL intensity was found by capturing an image of the irradiated sample and then determining the mean pixel count from an approximately 80 square pixel box using a freeware program, Rawnalyze [95]. Rawnalyze directly reads the pixel intensities without processing the image. Due to time restrictions, only the annealed imaging plates were measured as they showed the highest PSL intensity under X-rays.

Figure 7.8 shows the X-ray and neutron PSL intensities for all imaging plates tested. Europium only doped imaging plates (figure 7.8(a)) show a low PSL intensity under neutron irradiation at around $30-50 \%$ that of the commercial imaging plate. Imaging plates with converters containing enriched isotopes show a much higher PSL (figure 7.8(b)) under neutron irradiation than the 0.2 
\% Eu only imaging plate. Lithium-6 doped samples have a "raw" PSL intensity approximately $410 \%$ of the commercial plate, and this increases slightly (up to $450 \%$ ) with increasing converter fraction. Interestingly, the PSL intensity when irradiated with X-rays decreases with increasing converter fraction, whereas the PSL intensity under neutron irradiation increases. This probably reflects the counteracting tendencies of increasing neutron sensitivity with increasing converter fraction, but decreasing optical emission efficiency with increasing opacity. The $5 \%{ }^{10} \mathrm{~B}$ imaging plate shows the highest neutron PSL intensity at $560 \%$ of the commercial gadolinium-based imaging plate, which reflects efficient conversion of the neutrons into short range alpha particles by ${ }^{10}$ B. Gadolinium doped imaging plates also show a significant neutron PSL intensity, with the $5 \%$ Gd imaging plate having a relative PSL intensity of approximately $300 \%$.

However, the relative neutron PSL intensities given in figure 7.8 are overestimates of the absolute values. This is due to the optical filtration in the camera system being used having a different throughput for $\mathrm{BaFBr}: \mathrm{Eu}^{2+}$ as compared to $\mathrm{CsBr}: \mathrm{Eu}^{2+}$. As discussed in chapter 4.2 , the camera system is optimised for

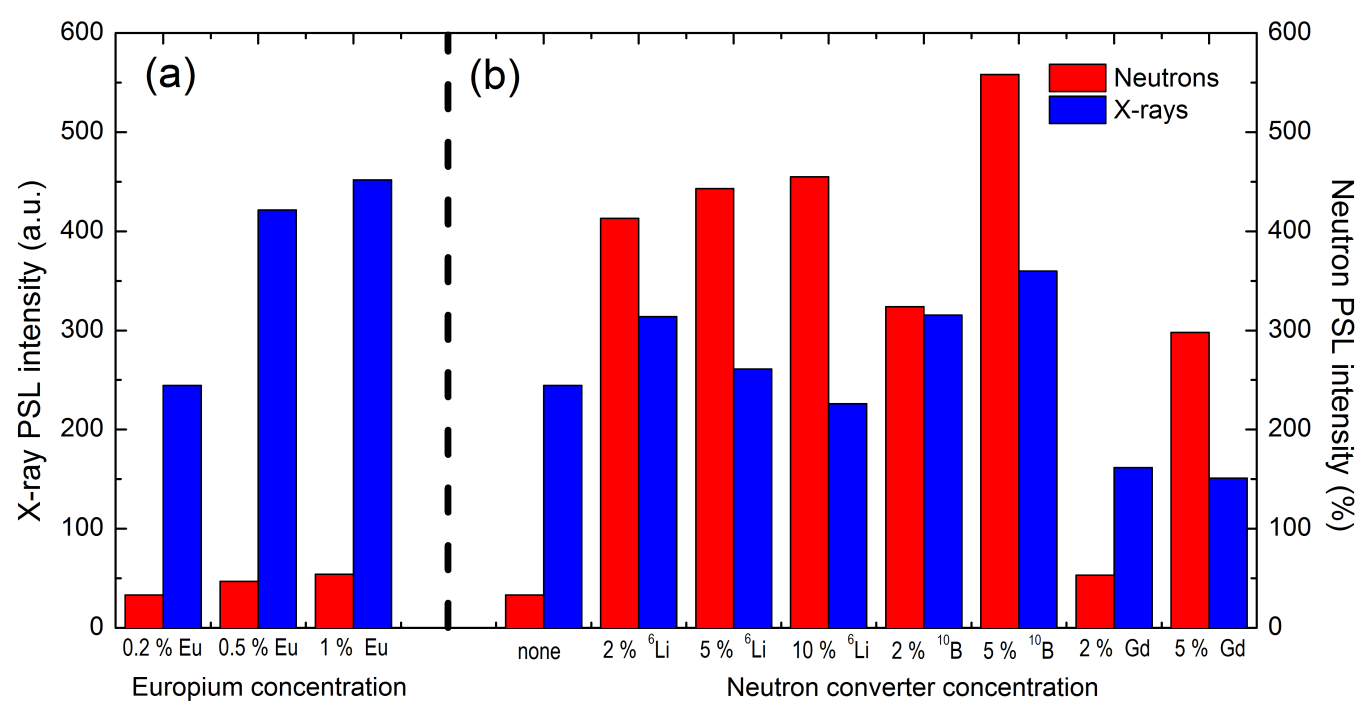

Figure 7.8: Neutron and X-ray PSL intensity scaled with thickness for (a) imaging plates doped with various europium concentrations, and (b) fixed europium concentration $(0.2 \mathrm{~mol} \%)$ with various additional neutron converters. The PSL intensity is as a percentage of the PSL intensity of the commercial Fuji neutron imaging plate. The X-ray PSL intensity scale is arbitrary. 


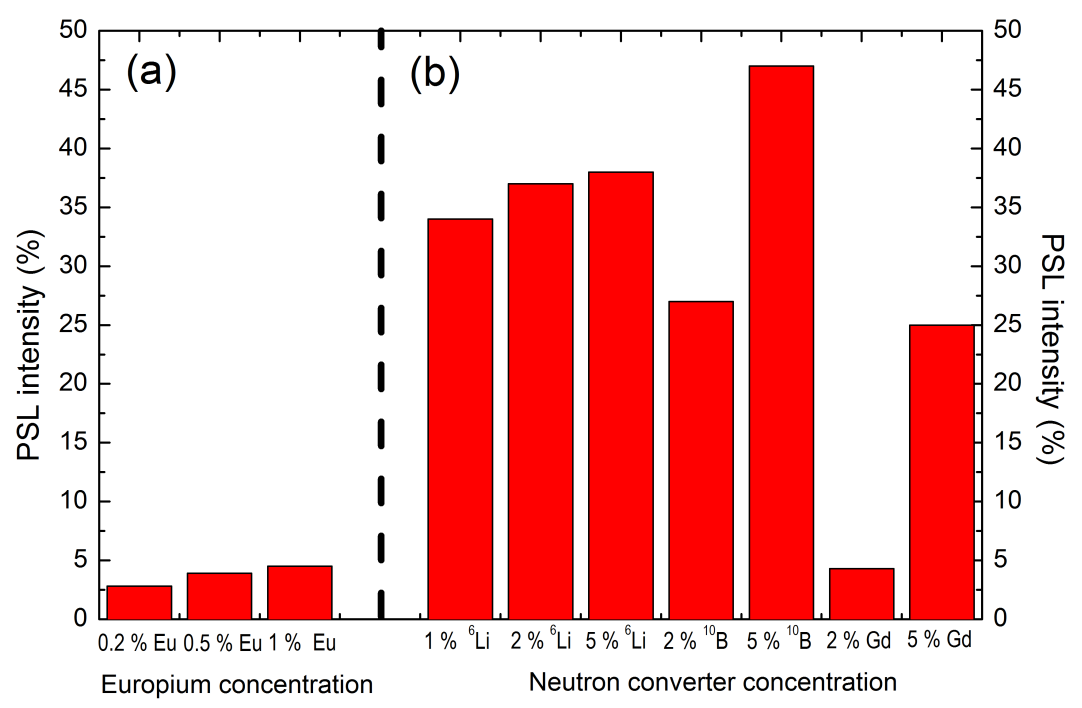

Figure 7.9: Corrected neutron PSL intensity for (a) imaging plates doped with various europium concentrations, and (b) fixed europium concentration (0.2 $\mathrm{mol} \%$ ) with various additional neutron converters as a percentage of PSL emission of the commercial imaging plate.

$\mathrm{CsBr}: \mathrm{Eu}^{2+}$ imaging plates due to their emission peak at $440 \mathrm{~nm}$ matching the peak of the blue channel transmission of the camera; the commercial neutron imaging plates based on BaFBr:Eu ${ }^{2+}$ have an emission at $390 \mathrm{~nm}$. The difference in detected PSL compared to emitted PSL was determined as follows. The PSL intensity as a function of wavelength was measured for both $\mathrm{CsBr}: \mathrm{Eu}^{2+}$ and $\mathrm{BaFBr}: \mathrm{Eu}^{2+}$. The intensity which is detected is given by,

$$
I=\int_{\lambda_{1}}^{\lambda_{2}} I_{0}(\lambda) T_{\text {filter }}(\lambda) T_{\text {camera }}(\lambda) d \lambda
$$

where $T_{\text {filter }}$ is the transmission characteristics of the BG12 filter and $T_{\text {camera }}$ is the transmission characteristics of the camera's blue dye. Taking the ratio of the area under the emission curve before and after filtration, it was found that 15.4 $\%$ and $1.3 \%$ of the light emitted by $\mathrm{CsBr}: \mathrm{Eu}^{2+}$ and $\mathrm{BaFBr}: \mathrm{Eu}^{2+}$ respectively was transmitted. Therefore, the BaFBr:Eu ${ }^{2+}$ PSL intensities need to be scaled up by a factor of 12 to be compared with those for $\mathrm{CsBr}: \mathrm{Eu}^{2+}$.

The neutron PSL intensities were recalculated, correcting for the optical filtration, and are shown in figure 7.9. The highest performing $\mathrm{CsBr}: \mathrm{Eu}^{2+}$ imaging plate $\left(5 \%{ }^{10} \mathrm{~B}\right)$ has a corrected PSL intensity of approximately $50 \%$ of the com- 


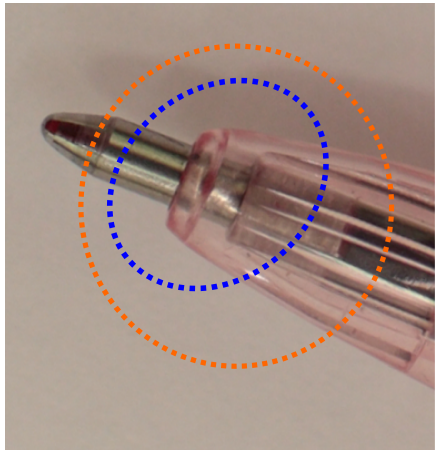

(a)

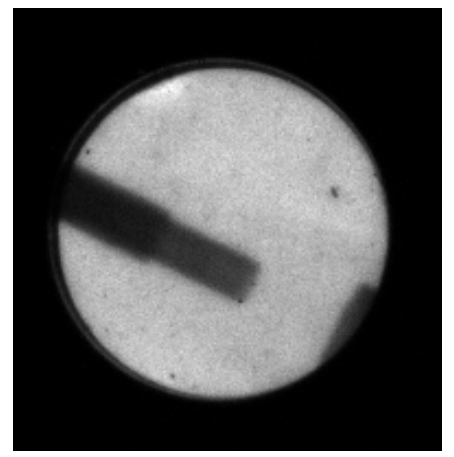

(b)

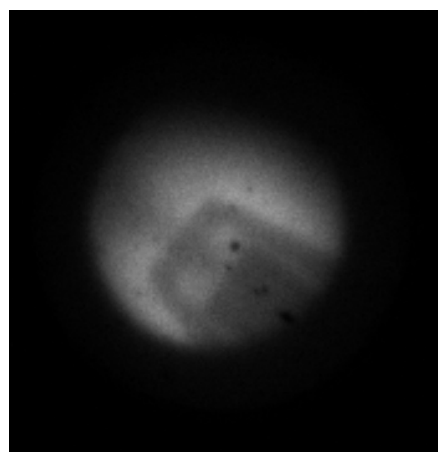

(c)

Figure 7.10: (a) Optical, (b) X-ray and (c) neutron images of a pen tip taken with the $1 \%{ }^{6} \mathrm{Li}$ imaging plate. The dashed circles on (a) represent the approximate locations of the X-ray (orange) and neutron (blue) image.

mercial Fuji imaging plate.

In addition to measuring the neutron PSL intensity, images were taken with the trial imaging plates. However, the neutron beam at the irradiation position only had a diameter of approximately $5 \mathrm{~mm}$ making imaging difficult. Figure 7.10 shows an optical, X-ray and neutron image of a pen tip taken with the $0.2 \%$ $\mathrm{Eu}^{2+} / 1 \%{ }^{6} \mathrm{Li}$ imaging plate. The X-ray and neutron images show the differences between the two radiation imaging methods, the X-ray image predominantly shows the metal nib, whereas the neutron image shows the plastic casing.

\subsection{Discussion}

$\mathrm{CsBr}: \mathrm{Eu}^{2+}$ with an added neutron converter shows good potential as a thermal neutron imaging plate. The best performing imaging plate, in terms of its PSL intensity under neutron irradiation, is one containing $5 \%$ enriched ${ }^{10} \mathrm{~B}_{2} \mathrm{O}_{3}$ as a neutron converter. This imaging plate has approximately $50 \%$ of the PSL intensity of a commercial Fuji neutron imaging plate for the same dose. Interestingly, imaging plates containing ${ }^{10} \mathrm{~B}_{2} \mathrm{O}_{3}$ as a neutron converter show no PSL under X-ray irradiation for both the as-made and hydrated imaging plates, but a significant PSL when the imaging plate is annealed. The reason for this could be that ${ }^{10} \mathrm{~B}_{2} \mathrm{O}_{3}$ is hygroscopic (more so than $\mathrm{EuBr}_{2}$ ), so for as-made and hydrated 
samples any available water is taken up by the ${ }^{10} \mathrm{~B}_{2} \mathrm{O}_{3}$. At high temperatures, such as during annealing, the water is released and is available to create the PSL active site. The optical quality of the imaging plates containing ${ }^{10} \mathrm{~B}_{2} \mathrm{O}_{3}$ as the neutron converter is poor due to the mismatch in refractive index. Additionally the powder tended to charge up when milling and sieving, making the recovery of enough powder to make an imaging plate difficult.

Enriched ${ }^{6} \mathrm{Li}_{3} \mathrm{CO}_{3}$ imaging plates also show a significant neutron PSL intensity at around $30-40 \%$ that of the commercial plate. These imaging plates were also of a reasonable optical quality and neutron images were able to be captured (see figure $7.10(\mathrm{c})$. The images have a reasonable resolution as the wall thicknesses of the pen, at around $0.5-1 \mathrm{~mm}$ are clearly defined.

The results show in figure 7.8(a) suggest that an approximate 2-fold increase in PSL intensity under neutron irradiation could be achieved by using a higher concentration of europium, say $1 \%$. This increased PSL is at the expense of optical transparency; an increase in europium concentration from $0.2 \%$ to $1 \%$ leads to the optical transparency decreasing by a factor of two. The europium-only doped imaging plates have a higher optical quality than any of imaging plates with added neutron converters, and they have the additional advantage that the europium is incorporated into the CsBr lattice making PSL more efficient. The main difficulty with adding neutron converters is the mismatch in refractive index between the $\mathrm{CsBr}$ and the neutron converter. Both the sensitivity and resolution would be improved if an enriched lithium or boron compound was used with a better refractive index match due to the decrease in scattering. Examples of lithium compounds which are better matched are $\mathrm{LiCl}$ and $\mathrm{LiBr}$ which have a refractive index of 1.66 and 1.78 respectively [92], compared to $\sim$ 1.70 for $\mathrm{CsBr}$ [45], although both are very hygroscopic. LiBr was incorporated as a neutron converter in section 7.2.1 and showed good PSL performance. An improved preparation process could involve milling the converter compound to a much smaller size, or incorporating them as nanoparticulates. The 
smaller size would reduce the optical scattering between neutron converter and $\mathrm{CsBr}$, leading to a much improved optical quality. Additionally, the range of conversion electrons is only 10's of microns, so having a smaller converter particle would lead to more neutron capture events occurring inside the converter actually reaching the PSL active site in the CsBr, maximising the PSL intensity. Improvements by optimising the hydration and annealing of the $\mathrm{CsBr}: \mathrm{Eu}^{2+}$ imaging plates would also increase the PSL intensity by maximising the number of PSL active sites, as was discussed in chapter 5 . 


\section{Chapter 8}

\section{Summary and outlook}

A new measurement process and method has been developed for determining both the conversion efficiency and stimulation energy of storage phosphor materials. This technique uses an integrating sphere to collect the light emitted by the sample after irradiation with a stimulating LED. The CE and SE values measured by this technique for $\mathrm{BaFBr}: \mathrm{Eu}^{2+}$ and $\mathrm{CsBr}: \mathrm{Eu}^{2+}$ imaging plates differ markedly from those found in literature. However, the technique is robust and so the differences in the values with the literature values are assumed to be caused by different measurement conditions, which are not explicitly specified in the literature reports. In particular mono-energetic versus poly-energetic $\mathrm{X}$-rays, different stimulation geometries, and different collection geometries are possible causes for the differences.

A portable storage phosphor imaging plate read-out system has been developed based on flood stimulation and commercially available digital cameras. The system is capable of recording images both with the pressed $\mathrm{CsBr}: \mathrm{Eu}^{2+}$ imaging plates developed in chapter 5 , as well as commercial $\mathrm{CsBr}: \mathrm{Eu}^{2+}$ or $\mathrm{BaFBr}: \mathrm{Eu}^{2+}$ imaging plates up to $240 \times 180 \mathrm{~mm}^{2}$ in size. While the main purpose in developing this system was to demonstrate and measure the performance of $\mathrm{X}$-ray storage phosphor materials developed in this thesis, the system also has a general applicability as a cost-effective portable $X$-ray imaging system. The principle advantages of this method over competing technologies are: 
- Portable and light-weight ( $7 \mathrm{~kg}$ ), and when coupled with a battery powered X-ray source (eg, the Golden Engineering XR150) and a laptop makes for a self-contained portable X-ray imaging system.

- Very cost-effective as it is based on off the shelf components and an easily obtainable semi-professional digital camera.

- The performance is comparable with laser-based read-out units, at a much lower cost.

- Is based on X-ray storage phosphor imaging plates and so has advantages over photographic film systems such as re-usability, no developing chemicals and directly obtainable digital images.

- Allows for registration of optical images with X-ray images.

Further work could be to develop the next generation read-out system, which would have improvements in design (for example, weight and aesthetics), the ability to take larger images (possibly by tiling) and improved image processing software.

A new preparation technique for producing transparent and semi-transparent $\mathrm{CsBr}: \mathrm{Eu}^{2+}$ storage phosphor imaging plates for X-ray radiography has been developed. The preparation process for producing transparent imaging plates involves, in the simplest process, milling $\mathrm{CsBr}$ and $\mathrm{EuBr}_{2}$ powders to a fine powder and then cold pressing into a disc using a hydraulic press. Surprisingly, as-made these imaging plates show a storage phosphor effect of up to $30 \%$ that of a commercial needle imaging plate, with an improved spatial resolution. Previous studies have found water plays a critical role in the PSL activation of the needle imaging plates. This is also true for the pressed imaging plates, and refinements to the basic process have been implemented. Subjecting the imaging plate to a humid atmosphere increases the PSL intensity to approximately two times that of a commercial needle imaging plate. However, water introduced to the pressed pellets causes a decrease in the transparency, as a 
recrystallisation of the imaging plate occurs.

A model for the generation of the PSL active site in these imaging plates has been suggested. In this model the PSL active site, $\left[\mathrm{Cs}_{\mathrm{v}}-\mathrm{Eu}^{2+}-\mathrm{Cs}_{\mathrm{v}}\left(\mathrm{H}_{2} \mathrm{O}\right)\right]$, is generated at the surface of the CsBr crystallites in two possible ways. If separate grains of $\mathrm{EuBr}_{2}\left(\right.$ or $\mathrm{EuBr}_{2} \cdot 1 \mathrm{H}_{2} \mathrm{O}$ ) are present, the surface water aids dissolution and distribution of the $\mathrm{Eu}^{2+}\left(\right.$ and $\left.\mathrm{Br}^{-}\right)$ions over the surface of the CsBr. In the second mechanism, $\mathrm{Eu}^{2+}-\mathrm{Cs}_{\mathrm{v}}$ agglomerates produced following thermomechanical sintering can dissociate, and the $\mathrm{Eu}^{2+}-\mathrm{Cs}_{\mathrm{v}}$ pairs migrate to the surface to combine with water molecules. Both processes occur at atomic steps on the surface of the $\mathrm{CsBr}$, and are progressively buried within the bulk of the regrowing $\mathrm{CsBr}$ crystallites during the regrowth process. The suggested model satisfactorily explains the results found in chapter 5 in a qualitative way. Further experimental work, particularly EPR spectroscopy, would be desirable for quantitative characterisation.

The best preparation process for the pressed imaging plates is likely to involve pre-hydrating the $\mathrm{CsBr}$ or $\mathrm{EuBr}_{2}$ powder with the required amount of water and then annealing under vacuum at approximately $150{ }^{\circ} \mathrm{C}$. This should result in a semi-transparent pellet with a conversion efficiency greater than that for the commercial needle imaging plates, and superior resolution.

The major effort in future synthesis refinement should be devoted towards optimising homogeneity and transparency, and size up-scaling. The first two may be further improved by implementing additional cycles of milling and pressing. Up-scaling will require a larger press coupled with exploration of the minimum pressure required to achieve a satisfactory transparency. An alternative pressing technique could be to compress the powders between two support sheets using rollers, which would also require investigations into the required pressure.

Dual-energy imaging based on colour discrimination with a two layered imaging plate was shown to be possible. A second imaging plate doped with 
monovalent indium was found to show a PSL effect in the green wavelength range, although the conversion efficiency was found to be much lower than for the europium doped imaging plates. A dual-layered structure was formed with an indium doped imaging plate as the top layer and an europium doped imaging plate as the bottom layer. Subtraction techniques were applied to the images recorded by this dual-layered structure, and partial material subtraction was achieved. Further investigations found that the PSL effect in the indium doped imaging plate is most likely not only due to the indium, but possibly due to other impurities or defects within the CsBr lattice, which are indirectly related to the indium content. Future work should investigate the use of another dopant which shows better colour separation and a PSL effect due to the dopant. One such dopant is monovalent gallium which shows a PSL effect separate from that of the $\mathrm{CsBr}$ impurities.

$\mathrm{CsBr}: \mathrm{Eu}^{2+}$ with an added neutron converter shows potential as a thermal neutron imaging plate. The best performing imaging plate was one with $5 \%{ }^{10} \mathrm{~B}_{2} \mathrm{O}_{3}$ added as a neutron converter, and had approximately $50 \%$ the PSL intensity of a commercial neutron imaging plate. Neutron imaging with these imaging plates was also demonstrated. The enriched isotope neutron converters showed a much higher PSL intensity under neutron irradiation than converters with a natural isotope abundance. The transparency of these imaging plates was poor, which is caused by the mismatch in refractive index between the neutron converter and $\mathrm{CsBr}$ leading to strong optical scattering. Better performing neutron imaging plates could be made by matching the refractive indices of the neutron converter and $\mathrm{CsBr}$. It may also be beneficial to reduce the size of the neutron converter particles to help with the optical transparency and to increase the number of ionising events being available for PSL.

The work presented in this thesis does not completely optimise the preparation process for production of transparent $\mathrm{CsBr}: \mathrm{Eu}^{2+}$ imaging plates. The interactions between $\mathrm{CsBr}, \mathrm{Eu}^{2+}$ ions and water molecules in the $\mathrm{CsBr}: \mathrm{Eu}^{2+}$ imaging 
plates are complex. However, a sound background on the preparation process and an interpretation has been given which can be used as the basis for further optimisation. 



\section{References}

[1] WWW.nist.gov/pml/data/xraycoef/index.cfm/.

[2] www.shiei.co.jp/english/c/nrt_photo.html.

[3] web.mit.edu/nrl/www/bnct/info/description/description. html.

[4] Sonoda, M., Takano, M., Miyahara, J. \& Kato, H. Computed radiography utilizing scanning laser stimulated luminescence. Radiology 148, 833-838 (1983).

[5] Yaffe, M. J. \& Rowlands, J. A. X-ray detectors for digital radiography. Physics in Medicine and Biology 42, 1-39 (1997).

[6] Lança, L. \& Silva, A. Digital radiography detectors - A technical overview: Part 1. Radiography 15, 58-62 (2009).

[7] Kasap, S. O., Zahangir Kabir, M. \& Rowlands, J. A. Recent advances in Xray photoconductors for direct conversion X-ray image detectors. Current Applied Physics 6, 288-292 (2006).

[8] medipix.web.cern.ch/medipix/index.php.

[9] Ludewig, E., Pees, M. \& Morgan, J. P. Clinical technique: Digital radiography in exotic pets - important practical differences compared with traditional radiography. Journal of Exotic Pet Medicine 21, 71-79 (2012). 
[10] Schweizer, S. \& Johnson, J. A. Fluorozirconate-based glass ceramic Xray detectors for digital radiography. Radiation Measurements 42, 632-637 (2007).

[11] Leblans, P. J., Willems, P. \& Alaerts, L. B. New needle-crystalline detector for X-ray computer radiography (CR). Journal of Nondestructive Testing 7 (2002).

[12] Edgar, A. Private communication (2009).

[13] Poludniowski, G., Landry, G., DeBlois, F., Evans, P. M. \& Verhaegen, F. Spekcalc : a program to calculate photon spectra from tungsten anode x-ray tubes. Physics in Medicine and Biology 54, N433-8 (2009).

[14] Lilley, J. Nuclear Physics, Principles and Applications (John Wiley \& Sons, England, 2001).

[15] Hussein, E. M. A. Handbook on Radiation Probing, Gauging, Imaging and Analysis, vol. 1 (Kluwer Academic Publishers, Netherlands, 2003).

[16] Knitel, M. New Inorganic Scintillators and Storage Phosphors for Detection of Thermal Neutrons. Ph.D. thesis, Delft University of Technology (1998).

[17] Blasse, G. \& Grabmaier, B. Luminescent Materials (Springer-Verlag, Berlin Heidelberg, 1994).

[18] Www.cancer-concerns.com/skin/skin1.htm.

[19] Schweizer, S. Physics and current understanding of X-ray storage phosphors. physica status solidi (a) 187, 335-393 (2001).

[20] Leblans, P., Vandenbroucke, D. \& Willems, P. Storage phosphors for medical imaging. Materials 4, 1034-1086 (2011).

[21] von Seggern, H. International Conference on Optical, Optoelectronic and Photonic Materials and Applications 2010 presentation (2010). 
[22] Edgar, A. et al. Photostimulated luminescence in a rare earth-doped fluorobromozirconate glass ceramic. Applied Physics Letters 75, 2386-2388 (1999).

[23] Hangleiter, T. et al. The X-ray storage phosphors RbI:Th+ ${ }^{+}$and KBr:In ${ }^{+}$and other $\mathrm{In}^{+}$and $\mathrm{Ga}^{+}$doped alkali halides. Proceedings of the International Conference on Inorganic Scintillators and their Applications, SCINT95 452 (1996).

[24] Weidner, M. et al. Luminescence spectroscopy of $\mathrm{Eu}^{2+}$ in $\mathrm{CsBr}: \mathrm{Eu}$ needle image plates (NIPs). Radiation Measurements 42, 661-664 (2007).

[25] Hackenschmied, P. et al. Storage performance of X-ray irradiated doped CsBr. Nuclear Instruments and Methods in Physics Research Section B 191, 163-167 (2002).

[26] Hackenschmied, P., Schierning, G., Batentschuk, M. \& Winnacker, A. Precipitation-induced photostimulated luminescence in $\mathrm{CsBr}: \mathrm{Eu}^{2+}$. Journal of Applied Physics 93, 5109-5112 (2003).

[27] Hesse, S. et al. CsEuBr ${ }_{3}$ crystal structure and its role in the photostimulation of CsBr:Eu' ${ }^{2+}$. Journal of Applied Physics 100, 083506-1-5 (2006).

[28] Hesse, S., Zimmermann, J., von Seggern, H., Ehrenberg, H. \& Fuess, H. The role of segregations and oxygen doping in the photostimulation mechanism of CsBr:Eu' ${ }^{2+}$. Radiation Measurements 42, 638-643 (2007).

[29] Appleby, G., Zimmermann, J., Hesse, S., Karg, O. \& von Seggern, H. Sensitization and radiation hardening of the photostimulable X-ray storage phosphor CsBr:Eu ${ }^{2+}$. Journal of Materials Science: Materials in Electronics 20, 54-58 (2009).

[30] Appleby, G. A., Zimmermann, J., Hesse, S., Karg, O. \& von Seggern, H. Sensitization of the photostimulable X-ray storage-phosphor CsBr:Eu ${ }^{2+}$ 
following room-temperature hydration. Journal of Applied Physics 105, 073511-073515 (2009).

[31] Appleby, G. A., Kroeber, P., Zimmermann, J. \& von Seggern, H. Influence of oxygen doping and hydration on photostimulated luminescence of $\mathrm{CsBr}$ and CsBr:Eu ${ }^{2+}$. Journal of Applied Physics 109, 073510-073515 (2011).

[32] Appleby, G. A., Zimmermann, J., Hesse, S. \& von Seggern, H. Photoluminescence and photostimulated luminescence of oxygen impurities in CsBr. Journal of Applied Physics 109, 013507-013505 (2011).

[33] Loncke, F. et al. Paramagnetic $\mathrm{Eu}^{2+}$ center as a probe for the sensitivity of CsBr X-ray needle image plates. Applied Physics Letters 92, 204102-1-3 (2008).

[34] Loncke, F. et al. Electron paramagnetic resonance study of a $\mathrm{Eu}^{2+}$ related defect in CsBr:Eu needle image plates for computed radiography. Spectrochimica Acta Part A: Molecular and Biomolecular Spectroscopy 69, 1322-1326 (2008).

[35] Loncke, F. et al. Temperature dependence of Eu-related EPR spectra in CsBr:Eu needle image plates. Physical Review B 79, 174102-1-9 (2009).

[36] Vrielinck, H. et al. Electron nuclear double resonance study of photostimulated luminescence active centers in $\mathrm{CsBr}: \mathrm{Eu}^{2+}$ medical imaging plates. Physical Review B 83, 054102-1-9 (2011).

[37] Rogulis, U. \& Schweizer, S. Photostimulated luminescence process in the X-ray storage phosphor CsBr:Ga. Journal of Applied Physics 87, 207-211 (2000).

[38] Kurata, N., Kubota, N., Takei, Y. \& Nanto, H. Eu-doped CsBr phosphor as a new optically-stimulable phosphor material for medical X-ray imaging sensor. Radiat Prot Dosimetry 119, 398-401 (2006). 
[39] Zorenko, Y. et al. Luminescence and storage properties of CsBr:Cu and CsBr:Cuo crystals. Physica Status Solidi A: Applications and Materials Science 202, 2537-2542 (2005).

[40] Zorenko, Y. et al. Growth and luminescence of CsBr:Cu crystals. Crystallography Reports 51, 329-333 (2006).

[41] Americium-241 Datasheet (Amersham International, England, 1974).

[42] Www. labsphere.com/uploads/technical-guides/

a-guide-to-integrating-sphere-theory-and-applications. $\mathrm{pdf}$.

[43] Www.cormusa.org/uploads/CORM_2009_SphCoat_

Springsteen.pdf.

[44] en.wikipedia.org/wiki/Roentgen_(unit).

[45] refractiveindex.info/?group=CRYSTALS\&material=CsBr.

[46] WWW.arcoptix.com/Fourier-transform-spectrometer-tutorial. $\mathrm{pdf}$.

[47] Weil, J. A., Bolton, J. R. \& Wertz, J. E. Electron paramagnetic resonance : elementary theory and practical applications (Wiley, New York, 1994).

[48] en.wikipedia.org/wiki/Electron_paramagnetic_resonance.

[49] science.howstuffworks.com/scanning-electron-microscope 2 . htm.

[50] Winch, N. M. \& Edgar, A. X-ray imaging using a consumer-grade digital camera. Nuclear Instruments and Methods in Physics Research Section A: Accelerators, Spectrometers, Detectors and Associated Equipment 654, 308-313 (2011). 
[51] Winch, N. M. \& Edgar, A. X-ray imaging using digital cameras. Proceedings of SPIE, Medical Imaging 2012: Physics of Medical Imaging 8313, 83135E-1-9 (2012).

[52] en.wikipedia.org/wiki/Bayer_filter.

[53] Www.insflug.org/raw.

[54] WwW.normankoren.com/Tutorials/MTF5.html.

[55] Dobbins, J. T., Samei, E., Ranger, N. T. \& Chen, Y. Intercomparison of methods for image quality characterization. II. Noise power spectrum. Medical Physics 33, 1466-1475 (2006).

[56] Winch, N. \& Edgar, A. Light scattering in glass ceramic x-ray storage phosphors. Journal of Applied Physics 105, 023506-023508 (2009).

[57] Lubinsky, A. R. et al. Scanning translucent glass-ceramic x-ray storage phosphors. Proceedings of SPIE. Medical Imaging 2010: Physics of Medical Imaging 7622, 76223W-1-8 (2010).

[58] Liu, H., Karellas, A., Harris, L. J. \& D'Orsi, C. J. Methods to calculate the lens efficiency in optically coupled CCD X-ray imaging systems. Medical Physics 21, 1193-1195 (1994).

[59] www.lsbu.ac.uk/water/vibrat.html.

[60] orgchem.colorado.edu/Spectroscopy/irtutor/aromaticsir. html.

[61] Krantz, M. \& Luty, F. Electronic and vibrational absorption and emission properties of F-center $\mathrm{OH}^{-}$defect pairs in cesium halides. Physical Review B 37, 8412-8416 (1988).

[62] Lutz, D. \& Christian, H. Raman and infrared spectra of barium and strontium halide monohydrates. Journal of Molecular Structure 96, 61-72 (1982). 
[63] Haschke, J. M. \& Eick, H. A. The preparation and some properties of europium bromides and hydrated bromides. Journal of Inorganic and Nuclear Chemistry 32, 2153-2158 (1970).

[64] Lutz, C. \& Christian, H. Librationnal modes of the water molecules in barium and strontium monohydates $\mathrm{MX}_{2} \cdot 1 \mathrm{H}_{2} \mathrm{O}(\mathrm{M}=\mathrm{Sr}, \mathrm{Ba} ; \mathrm{X}=\mathrm{Cl}, \mathrm{Br}, \mathrm{I})$. Journal of Molecular Structure 101, 199-212 (1983).

[65] Arsic, J., Reynhout, I., van Enckevort, W. \& Vlieg, E. Equilibrium morphologies and thermal roughening of cesium halides. Journal of Crystal Growth 245, 171-179 (2002).

[66] Arsic, J., Reynhout, I. C., van Enckevort, W. J. P. \& Vlieg, E. Growth and characterization of cesium halides with cubic morphologies. Journal of Crystal Growth 253, 472-480 (2003).

[67] Lopez, F. J., Murrieta S., H., Hernandez A, J. \& Rubio O, J. Optical absorption and luminescence investigations of the precipitated phases of $\mathrm{Eu}^{2+}$ in $\mathrm{NaCl}$ and $\mathrm{KCl}$ single crystals. Physical Review B 22, 6428-6439 (1980).

[68] Abragam, A. \& Bleaney, B. Electron paramagnetic resonance of transition ions (Dover Publications, United States, 1987).

[69] Stoll, S. \& Schweiger, A. EasySpin, a comprehensive software package for spectral simulation and analysis in EPR. Journal of Magnetic Resonance 178, 42-55 (2006).

[70] Savel'ev, V. P., Avdonin, V. P., Dugarova, L. D., Nedashkovskii, A. P. \& Plachenov, B. T. Aggregation of $\mathrm{Eu}^{2+} \mathrm{V}^{-}$centres in europium-activated alkali halide crystals. Soviet Physics Solid State 16, 700-702 (1974).

[71] Www.docstoc.com/docs/25929035/Table-of-Fourier-Transform-Pairs

[72] Fuchs, M., Hackenschmied, P. \& Hell, E. Method for the production of and 
protective layer for a layer of luminescent material. United States Patent US 7,288,769 B2 (2007).

[73] McAdams, H. P., Samei, E., Dobbins Iii, J., Tourassi, G. D. \& Ravin, C. E. Recent advances in chest radiography. Radiology 241, 663-683 (2006).

[74]

science.howstuffworks.com/transport/flight/modern/ airport-security 4. htm

[75] Bartle, C. M., Kroger, C. \& West, J. G. New uses of X-ray transmission techniques in the animal-based industries. Radiation Physics and Chemistry 71, 843-851 (2004).

[76] Alvarez, R. E. \& Macovski, A. Energy-selective reconstructions in X-ray computerised tomography. Physics in Medicine and Biology 21, 733-744 (1976).

[77] Lehmann, L. A. et al. Generalized image combinations in dual KVP digital radiography. Medical Physics 8, 659-667 (1981).

[78] Fabbri, S. et al. Signal-to-noise ratio evaluation in dual-energy radiography with synchrotron radiation. Physics in Medicine and Biology 47, 4093-4105 (2002).

[79] Marziani, M., Taibi, A., Tuffanelli, A. \& Gambaccini, M. Dual-energy tissue cancellation in mammography with quasi-monochromatic X-rays. Physics in Medicine and Biology 47, 305-313 (2002).

[80] physics.nist.gov/PhysRefData/Xcom/html/xcoml.html.

[81] Thoms, M., von Seggern, H. \& Winnacker, A. Optical and thermal properties of electron- and hole-trapping sites in the X-ray storage phosphor $\mathrm{RbI}: \mathrm{X}\left(\mathrm{X}=\mathrm{Tl}^{+}, \mathrm{In}^{+}, \mathrm{Pb}^{2+}, \mathrm{Eu}^{2+}\right)$. Journal of Applied Physics 76, 1800-1808 (1994). 
[82] Zazubovich, S. G., Kondrat'ko, M. Y., Nikotin, O. P., Osminin, V. S. \& Teodorovich, O. A. Anisotropic activator color centers in spontaneously colored gallium-activated cesium bromide and iodide crystals. Sbornik Trudov - Kafedra Yadernoi Fiziki i Radiatsionnoi Khimii, Leningradskii Tekhnologicheskii Institut imeni Lensoveta 2, 16-34 (1971).

[83] Mikhal'chenko, G. A., Kondrat'ko, M. Y. \& Vinogradova, T. P. Stable color centers in cesium halides. Zhurnal Prikladnoi Spektroskopii 4, 278-279 (1966).

[84] Ishigaki, T., Sakuma, S., Horikawa, Y., Ikeda, M. \& Yamaguchi, H. One-shot dual-energy subtraction imaging. Radiology 161, 271-273 (1986).

[85] WWw.ncnr.nist.gov/resources/n-lengths/list.html.

[86] Lehmann, E. H., Vontobel, P., Frei, G. \& Brnnimann, C. Neutron imaging detector options and practical results. Nuclear Instruments and Methods in Physics Research Section A: Accelerators, Spectrometers, Detectors and Associated Equipment 531, 228-237 (2004).

[87] physics.nist.gov/PhysRefData/Star/Text/ESTAR.html.

[88] www.ansto.gov.au/discovering_ansto/anstos_research_ reactor

[89] Anderson, I. S., McGreevy, R. L. \& Bilheux, H. Z. Neutron Imaging and Applications (Springer, New York, 2009).

[90] Kienle, F. A portable imaging plate sensitivity meter. Tech. Rep., Victoria University of Wellington (2005).

[91] Meschan, I., Oddie, T. H. \& Brucer, M. The utilization of europium 152-154 in a cervical carcinoma applicator. Radiology 67, 422-423 (1956).

[92] CRC Handbook of Chemistry and Physics (CRC Press, Ohio, 1973), 54th edition edn. 
[93] www.detectors.saint-gobain.com/uploadedFiles/ SGdetectors/Documents/Product_Data_Sheets/ BrilLanCe350-data-sheet.pdf.

[94] webbook.nist.gov/cgi/cbook.cgi? ID =B6000502\&Mask=80.

[95] dave-anderson-photo.com/blog/2010/08/23/ gabor-rawnalyze-author-rip/. 


\section{Appendix A}

\section{LED timer circuit}

The electronics for the LED timer circuit were designed by Mr T. Exley.

\section{LED Timer - Description}

The LED Timer circuit consists of an LM555 timer that drives one of four constant current LED drivers. The power supply input will accept 8 to 24VDC.

The LED current limits are set to $700 \mathrm{~mA}$ for all LEDs. The voltage drop across each LED is approximately $2.5 \mathrm{~V}$, which allows for two LEDs wired in series per driver when the board is supplied with approx 8 volts. The two sets of drivers for the Red and Green LEDs allows for more than two LEDs to be driven per driver when the supply is at its minimum.

When supplying the board with $12 \mathrm{~V}$ and with two LEDs (in series) per driver, the LM317s will have to dissipate approx $3 \frac{1}{2} \mathrm{~W}$, hence the need for heatsinks on the LM317s.

To select which LED is turned on by the timer, wire PL10 as per the table below:

$\begin{array}{ll}\text { LED } & \text { Wire PL10-6 to } \\ \text { Red } & \text { PL10-1 } \\ \text { Green } & \text { PL10-2 } \\ \text { Blue } & \text { PL10-3 } \\ \text { White } & \text { PL10-4 }\end{array}$

To select a time that the LEDs will be on for, wire PL5A and PL5B as per the table below:

$\begin{array}{ll}\text { Timer Setting } & \text { Wire PL5A-1 to } \\ 200 \mathrm{~ms} & \text { PL5A-2 } \\ 500 \mathrm{~ms} & \text { PL5A-3 } \\ 1 \mathrm{~s} & \text { PL5A-4 } \\ 2 \mathrm{~s} & \text { PL5A-5 } \\ 5 \mathrm{~s} & \text { PL5A-6 } \\ 10 \mathrm{~s} & \text { PL5B-1 } \\ 20 \mathrm{~s} & \text { PL5B-2 } \\ 50 \mathrm{~s} & \text { PL5B-3 } \\ 100 \mathrm{~s} & \text { PL5B-4 } \\ 200 \mathrm{~s} & \text { PL5B-5 } \\ 500 \mathrm{~s} & \text { PL5B-6 }\end{array}$




\section{LED Timer Connectors}

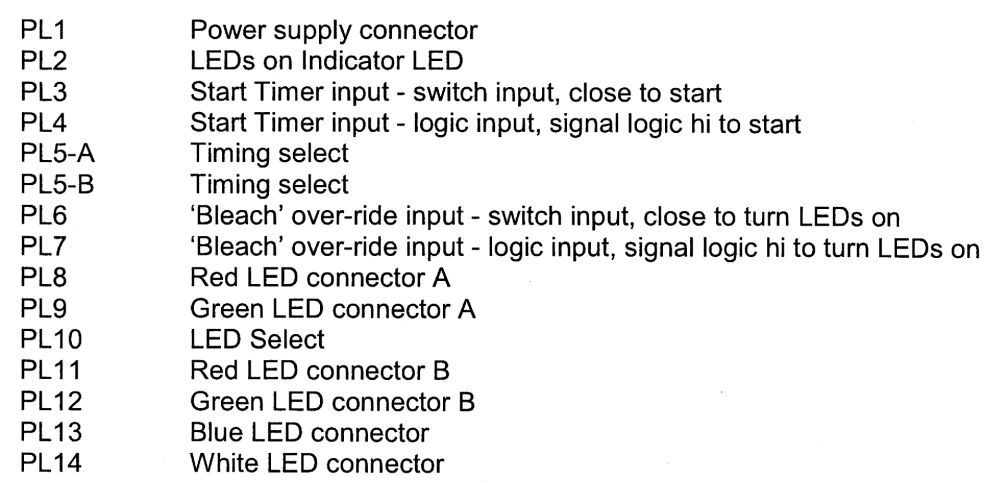

\begin{tabular}{|c|c|c|c|c|c|c|c|}
\hline Power & PL1 & 1 & GND & Timing Select & PL5-A & 1 & GND \\
\hline & & 2 & Supply V+ & & & 2 & $200 \mathrm{~ms}$ \\
\hline & & & & & & 3 & $500 \mathrm{~ms}$ \\
\hline & & & & & & 4 & 1s \\
\hline & & & & & & 5 & $2 s$ \\
\hline LEDs On & PL2 & 1 & $+5 \mathrm{~V}$ via $1 \mathrm{~K}$ res. & & & 6 & $5 s$ \\
\hline & & 2 & Open collector & Timing Select & PL5-A & 1 & $10 \mathrm{~s}$ \\
\hline & & & & & & 2 & $20 s$ \\
\hline & & & & & & 3 & $50 s$ \\
\hline & & & & & & 4 & $100 \mathrm{~s}$ \\
\hline & & & & & & 5 & $200 s$ \\
\hline & & & & & & 6 & $500 s$ \\
\hline Red LED & PL8 & 1 & LED Anode & & & & \\
\hline & & 2 & LED Cathode & & & & \\
\hline & & & & LED Select & PL10 & 1 & Red LEDs \\
\hline & PL11 & 1 & LED Anode & & & 2 & Green LEDs \\
\hline & & 2 & LED Cathode & & & 3 & Blue LEDs \\
\hline & & & & & & 4 & White LEDs \\
\hline Green LED & PL8 & 1 & LED Anode & & & $\begin{array}{l}5 \\
6\end{array}$ & $\begin{array}{l}\text { not connected } \\
\text { LED Select }\end{array}$ \\
\hline & & 2 & LED Cathode & & & & \\
\hline & PL11 & 1 & LED Anode & Start timer & PL3 & 1 & $+5 \mathrm{~V}$ \\
\hline & & 2 & LED Cathode & & & 2 & Start timer input \\
\hline & & & & & PL4 & 1 & GND \\
\hline Blue LED & PL8 & $\begin{array}{l}1 \\
2\end{array}$ & $\begin{array}{l}\text { LED Anode } \\
\text { LED Cathode }\end{array}$ & & & 2 & Start timer input \\
\hline & & & & Bleach & PL6 & 1 & $+5 \mathrm{~V}$ \\
\hline White LED & PL8 & $\begin{array}{l}1 \\
2\end{array}$ & $\begin{array}{l}\text { LED Anode } \\
\text { LED Cathode }\end{array}$ & & & 2 & LED "on" input \\
\hline & & & & & PL7 & $\begin{array}{l}1 \\
2\end{array}$ & $\begin{array}{l}\text { GND } \\
\text { LED "on" input }\end{array}$ \\
\hline
\end{tabular}




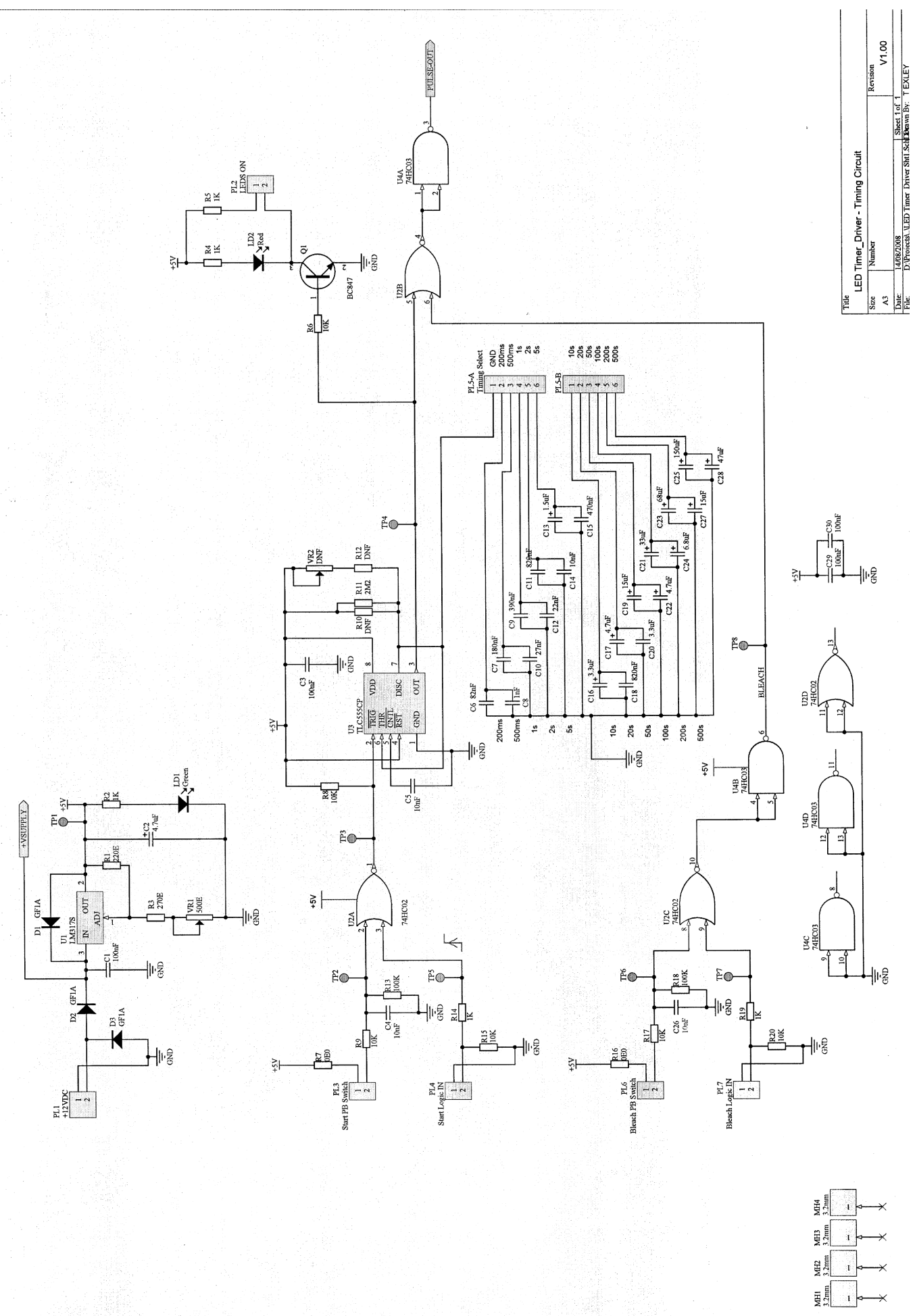



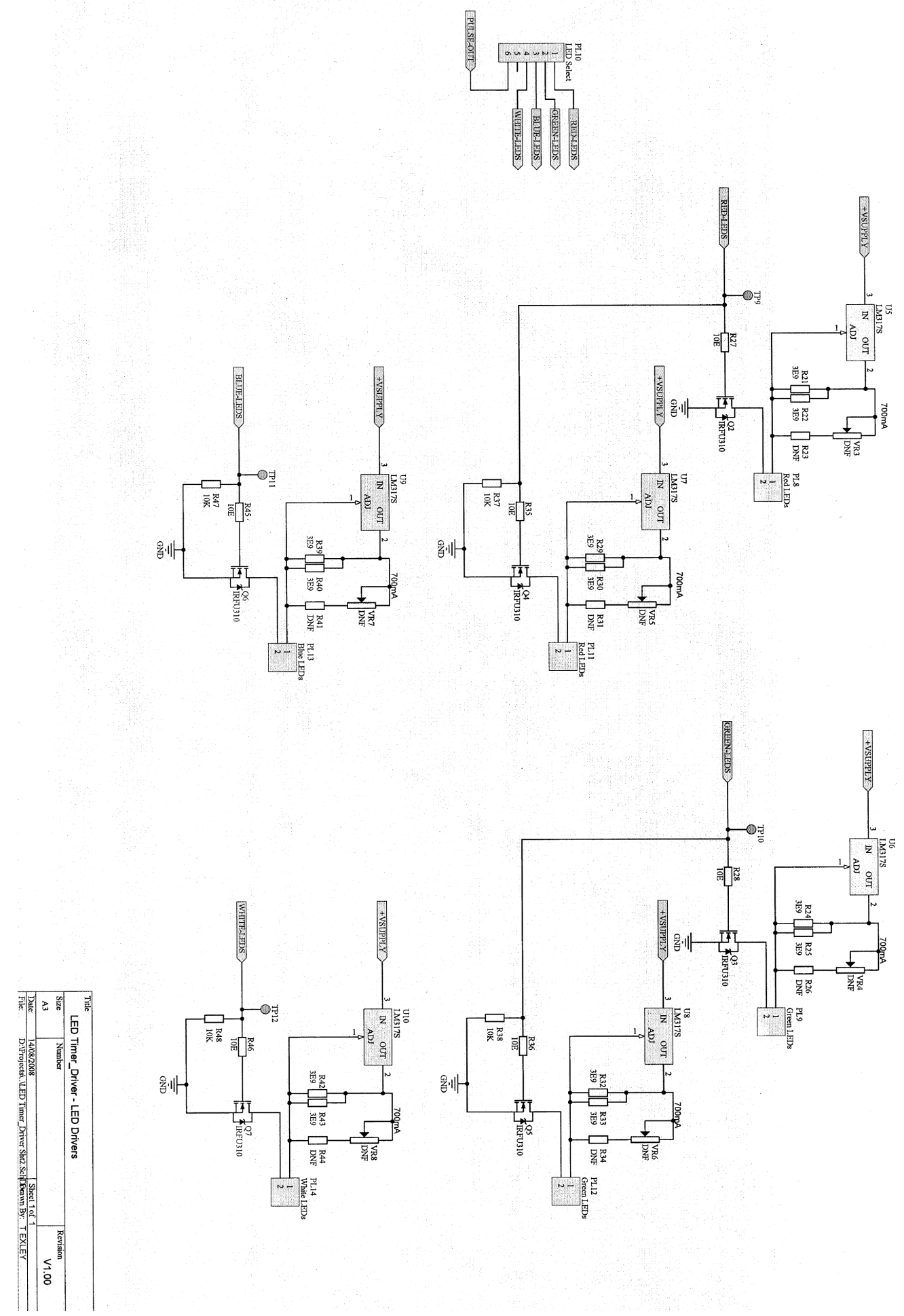


\section{Appendix B}

\section{Modulation transfer function}

\section{B.1 Optical MTF for the camera system}

The PSL intensity from an image of the test grid which reaches the imaging sensor can be approximated (in one dimension) for each periodic section as a Fourier series of the form,

$$
y(l)=a_{0}+\sum_{m=1}^{\infty} a_{m} \sin (m k l),
$$

where $k$ is the spatial frequency ( $k=\frac{2 \pi}{\lambda}=2 \pi \times$ line pairs $/ m m, \lambda$ is the period), $m$ the harmonic number and $l$ the position along the length of the imaging sensor.

The sensor has a series of pixels of length $L$ and acts to integrate the signal over the length of one pixel. The output $y$ as a function of the pixel number $(n=1,2,3, \ldots)$ is,

$$
y(n)=\int_{L_{0}+n L}^{L_{0}+(n+1) L} y(l) d l,
$$

where $L_{0}$ is a constant which represents the fact that the PSL pattern and the pixel pattern of the sensor do not necessarily line up with each other. The 
following analysis determines the result of equation B.2.

$$
\begin{aligned}
y(n) & =a_{0} L+\sum_{m=1}^{\infty} a_{m} \int_{L_{0}+n L}^{L_{0}+(n+1) L} \sin (m k l) d l \\
& =a_{0} L+\sum_{m=1}^{\infty} \frac{a_{m}}{m k}[-\cos (m k l)]_{L_{0}+n L}^{L_{0}+(n+1) L}, \\
& =a_{0} L+\sum_{m=1}^{\infty} \frac{a_{m}}{m k}\left[\cos \left(m k\left\{L_{0}+n L\right\}\right)-\cos \left(m k\left\{L_{0}+(n+1) L\right\}\right)\right] .
\end{aligned}
$$

Using,

$$
\cos A-\cos B=-2 \sin \left(\frac{A+B}{2}\right) \sin \left(\frac{A-B}{2}\right),
$$

leads to,

$$
\begin{aligned}
y(n) & =a_{0} L+\sum_{m=1}^{\infty} \frac{-a_{m}}{\left(\frac{m k}{2}\right)} \sin \left(m k\left\{L_{0}+\left(n+\frac{1}{2}\right) L\right\}\right) \sin \left(\frac{-m k L}{2}\right), \\
& =a_{0} L+\sum_{m=1}^{\infty} \frac{a_{m} \sin \left(\frac{m k L}{2}\right)}{\left(\frac{m k}{2}\right)} \sin \left(m k\left\{L_{0}+\frac{1}{2} L\right\}+m k n L\right), \\
& =a_{0} L+\sum_{m=1}^{\infty} \frac{a_{m} \sin \left(\frac{m k L}{2}\right)}{\left(\frac{m k}{2}\right)} \sin (m k n L+\phi),
\end{aligned}
$$

where $\phi=m k\left(L_{0}+\frac{1}{2} L\right)$. The PSL signal is perceived by the sensor as a sine wave in the variable $n$ with an amplitude $A$ which varies as,

$$
A=a_{m} \frac{\sin x}{x}
$$

where $x=\frac{m k L}{2}$, and $\frac{\sin x}{x}$ is known as the sinc function.

To determine the optical modulation transfer functions of the camera read-out system (for example in figure 4.5) an image of a spatially varying sinusoidal test chart is obtained. As the object being imaged is sinusoidal, the image seen by the pixels is described by the result in equation B.5 with $m=1$. Therefore, if the MTF was only due to the pixelation of the relevant sensor the curve should 
have a sinc function shape, which falls to zero at,

$$
\frac{k L}{2}=\pi
$$

or,

$$
\text { Line pairs per } m m=\frac{1}{L} \text {. }
$$

\section{B.2 Fitting data with a sine wave}

The X-ray MTFs (for example figure 4.5) are measured using a lead grid which produces a square-wave pattern. The MTF is determined by least squares fitting the data for each spatial frequency with a sine wave and extracting the amplitude. This method gives the same results as Fourier transforming the data would, as proved by the following argument.

\section{Fourier series}

The PSL intensity transmitted through an object (such as the lead grid) is assumed to be of the form,

$$
y(l)=a_{0}+\sum_{m=1}^{\infty} a_{m} \sin \left(\frac{2 \pi m l}{\lambda}\right)
$$

where $\frac{2 \pi}{\lambda}=k$ is the spatial frequency, $m$ the harmonic number, and $a_{m}$ is the amplitude of interest for determining the MTF. The amplitude $a_{m}$ can be found by multiplying both sides of equation B.9 by,

$$
\sin \left(\frac{2 \pi n l}{\lambda}\right)
$$


where $n$ is an integer greater than one, and integrating over the period 0 to $\lambda$. That is,

$$
\begin{aligned}
\int_{0}^{\lambda} y(l) \sin \left(\frac{2 \pi n l}{\lambda}\right) d l & =\int_{0}^{\lambda} a_{0} \sin \left(\frac{2 \pi n l}{\lambda}\right) d l \\
& +\sum_{m=1}^{\infty} \int_{0}^{\lambda} a_{m} \sin \left(\frac{2 \pi m l}{\lambda}\right) \sin \left(\frac{2 \pi n l}{\lambda}\right) d l
\end{aligned}
$$

The first term in the above equation integrates to zero, and the second term is only non-zero when $m=n$ therefore,

$$
\begin{aligned}
\int_{0}^{\lambda} y(l) \sin \left(\frac{2 \pi n l}{\lambda}\right) d l & =\int_{0}^{\lambda} a_{n} \sin ^{2}\left(\frac{2 \pi n l}{\lambda}\right) d l \\
& =\frac{a_{n} \lambda}{2}
\end{aligned}
$$

Rearranging for $a_{n}\left(=a_{m}\right)$ gives,

$$
a_{n}=\frac{1}{\lambda} \int_{0}^{\lambda} y(l) \sin \left(\frac{2 \pi n l}{\lambda}\right) d l .
$$

\section{Least squares fitting}

The grid data for a specific set of spatial frequencies is least squares fitted with an equation of the form,

$$
y_{f i t}(l)=A \sin \left(\frac{2 \pi l}{\lambda}\right)
$$

Least squares fitting is performed by minimising the sum of the square residuals ( $S$, equation B.15) over the period of the data,

$$
S=\sum_{l=0}^{\lambda}\left[y(l)-y_{f i t}(l)\right]^{2} .
$$


The residuals are minimised by differentiating equation B.15 with respect to amplitude $A$ and then setting equal to zero. That is,

$$
\begin{aligned}
S & =\sum_{l=0}^{\lambda}\left[y(l)-A \sin \left(\frac{2 \pi l}{\lambda}\right)\right]^{2}, \\
\frac{d}{d A} S & =\sum_{l=0}^{\lambda} 2\left[y(l)-A \sin \left(\frac{2 \pi l}{\lambda}\right)\right] \sin \left(\frac{2 \pi l}{\lambda}\right) .
\end{aligned}
$$

Assuming that the sum is continuous, and therefore an integral over 0 to $\lambda$ gives,

$$
\begin{aligned}
\frac{d}{d A} S & =\int_{0}^{\lambda} y(l) \sin \left(\frac{2 \pi l}{\lambda}\right) d l-2 A \int_{0}^{\lambda} y(l) \sin ^{2}\left(\frac{2 \pi l}{\lambda}\right) d l \\
& =\int_{0}^{\lambda} y(l) \sin \left(\frac{2 \pi l}{\lambda}\right) d l-2 A\left[t \frac{1}{2} l-\frac{1}{4} \sin \left(\frac{4 \pi l}{\lambda}\right)\right]_{0}^{\lambda} \\
& =\int_{0}^{\lambda} y(l) \sin \left(\frac{2 \pi l}{\lambda}\right) d l-A \lambda .
\end{aligned}
$$

Setting equation $\mathrm{B} .18$ to zero gives,

$$
\int_{0}^{\lambda} y(l) \sin \left(\frac{2 \pi l}{\lambda}\right) d l=A \lambda
$$

and rearranging,

$$
A=\frac{1}{\lambda} \int_{0}^{\lambda} y(l) \sin \left(\frac{2 \pi l}{\lambda}\right) d l
$$

Equation B.20 is the same as equation B.13 for the Fourier series method, therefore the two techniques are equivalent. 



\section{Appendix C}

\section{List of publications}

The following publications are based on the work described in this thesis.

1. Winch, N. M. \& Edgar, A. X-ray imaging using a consumer-grade digital camera. Nuclear Instruments and Methods in Physics Research Section A: Accelerators, Spectrometers, Detectors and Associated Equipment 654, 308-313 (2011).

2. Winch, N. M. \& Edgar, A. X-ray imaging using digital cameras. Proceedings of SPIE, Medical Imaging 2012: Physics of Medical Imaging 8313, 83135E-1-9 (2012).

3. Winch, N. M. \& Edgar, A. Transparent caesium bromide storage-phosphors for radiation imaging. physica status solidi (a) 209, 2427-2432 (2012). 